\title{
CLINICAL SCIENCES AND THERAPEUTICS FOR HEALTH
}

\section{CS/TH-0-1: THE EFFICACY OF A 12 WEEK EXERCISE INTERVENTION IN ADOLESCENTS AGED 11-16 YEARS WITH AUTISM SPECTRUM DISORDER}

Natalia Neophyłou, Kerith Aginsky, Natalie Benjamin

Centre for Exercise Science and Sports Medicine, School of Therapeutic Sciences, Faculty of Health Sciences, University of the Witwatersrand, Johannesburg, South Africa

Autism Spectrum Disorder (ASD) is a neurodevelopmental disorder, which often results in an array of motor impairments including poor posture, gait, coordination and muscle tone. Good posture is important not only in protecting individuals from injury, but also in improving the efficiency of performance to maximal capacity. It is therefore important to be able to assess posture and improve postural deficits where necessary, particularly in individuals with ASD, as postural information in this population is insufficient. A randomised control trial was conducted to determine the efficacy of a 12 week exercise intervention by assessing the change in posture, body composition and physical fitness, pre- and post-exercise intervention in adolescents with ASD. All participants were tested pre- and post-intervention. The intervention group $(n=16)$ participated in the exercise intervention bi-weekly for 12 weeks, while the control group $(n=11)$ received standard care. Posture was assessed using a posture grid, and scores out of 10 were given per body area, where good posture $=10$, average posture $=5$ and poor posture $=0$. Body composition and physical fitness were assessed using the Brockport Physical Fitness Test. Overall compliance to the intervention was $88.78 \%$. There was a significant increase in the overall posture scores $(p=0.0004)$, specifically in the ankle area $(p=0.0183)$ in the intervention group. There was a significant reduction in body mass index $(p=0.0130)$ post intervention, and significant decreases in resting systolic blood pressure $(p=0.0069)$, and systolic blood pressure taken one minute following exercise $(p=0.0007)$. A significant decrease in resting heart rate $(p=0.0046)$, as well as in heart rate taken one minute following exercise $(p=0.0096)$ was also seen. Handgrip strength significantly increased in the non-dominant hand $(p=0.0289)$. The intervention group improved significantly in the amount of curl-ups they were able to perform following the intervention ( $p=0.0094)$.

Key words: Autism Spectrum Disorder, Exercise Intervention, Posture, Physical Fitness

\section{CS/TH-O-2:SPATIAL PATTERNS AND TRENDS OF MATERNAL MORTALITY OVER A FIVE YEAR PERIOD AND THEIR ASSOCIATED RISK FACTORS IN IFAKARA HEALTH AND DEMOGRAPHIC SURVEILLANCE SITE}

Alfred Manyeh ${ }^{1,2}$, Alfred Kwesi Manyeh ${ }^{3}$, Gill Nelson', Rose Nathan ${ }^{4}$

'School of Public Health, Faculty of Health Sciences, University of the Witwatersrand, Johannesburg, South Africa, ${ }^{2}$ Ifakara Health Institute, ${ }^{3}$ Dodowa Health Research Centre, Ghana, ${ }^{4}$ Ifakara Health and Demographic Surveillance System Site, Tanzania

Maternal mortality is the subject of the United Nations' fifth Millennium Development Goal, which is to reduce the maternal mortality ratio by three quarters from 1990 to 2015. Knowing the spatial patterns and risk factors associated with maternal mortality in developing countries will help target scarce resources to high risk areas. Secondary data of women aged 15 to 49 years from 2005 to 2010 was analysed. An inverse distance weighted method of interpolation was used to assess spatial patterns of maternal mortality. Cox proportional hazards regression was used to identify risk factors. Maternal deaths declined from 90.42 in 2006 to 57.42 in 2010 with marked geographical differences in patterns. The main causes of death were eclampsia $(23 \%)$, haemorrhage $(22 \%)$ and abortion related complications (10\%). There was reduced risk of $82 \%$ (HR: $0.18,95 \% \mathrm{Cl}: 0.05-0.74$ ) and 78\% (HR: 0.22, 95\% Cl: 0.05 - 0.92) for women aged 20-29 and 30-39 years, respectively, compared with those $<20$ years. While being married had a protective effect of $94 \%$ (HR: $0.06,95 \% \mathrm{Cl}: 0.01-0.51$ ) compared to being single, widows had an increased risk of $813 \%$ (HR: $9.13,95 \% \mathrm{Cl}$ : (1.017 - 81.942). There has been decline in maternal mortality in rural southern Tanzania since 2006, with geographical differences in pattern of death. Eclampsia, haemorrhage and abortion-related complications are the leading causes of death, with risk factors being younger than 20 years, being single or widowed, and low socioeconomic status.

Key words: Maternal mortality, spatial pattern, verbal autopsy, Tanzania 


\section{CS/TH-O-3: THE ACCURACY OF BLOOD PRESSURE DEVICES IN PAEDIATRIC RENAL OUTPATIENTS}

Nokukhaya Mwandla', Udai Kala²

'Department of Paediatrics, School of Clinical Medicine, Faculty of Health Sciences, University of Witwatersrand, Johannesburg, South Africa, ${ }^{2} \mathrm{Head}$ of Department, Paediatric Nephrology, Chris Hani Baragwanath Academic Hospital, Johannesburg, South Africa

Childhood hypertension is an important condition most often due to secondary causes. The accurate measurement of blood pressure (BP) is imperative in child patients with risks for or known with hypertension. The purpose of this study was to determine the accuracy of aneroid and automated machines versus the mercury gold-standard in BP determination. Methods: In this prospective comparative study, 94 paediatric renal outpatients had BP taken in a random order with Welch Allyn aneroid (๑), Dinamap Pro $100 \odot$ and Omron HEM 907@ manometers. Two trained observers obtained the manual device readings independently. Inter-device differences were used to validate each of the devices by the British Hypertension Society (BHS) and the Association for the Advancement of Medical Instrumentation (AAMI) protocol standards. Results: The aneroid manometer overestimated SBP and DBP by $2 \mathrm{mmHg}(p=0.006)$ and $5 \mathrm{mmHg}(p<0.001)$, respectively. It fulfilled validation criteria for SBP according to AAMI only. The Dinamap Pro 100 overestimated SBP and underestimated DBP by $5 \mathrm{mmHg}(p<0.001)$ and $8 \mathrm{mmHg}(p<0.001)$, respectively. The Dinamap did not fulfil criteria for both AAMI and BHS. The Omron HEM 907 was accurate for SBP, but overestimated DBP by $5 \mathrm{mmHg}$ ( $p=0.001$ ), on aggregates. It met criteria for SBP according to BHS criteria only. Inherent BP fluctuations in individuals may have affected reliability. However, the interobserver agreement for all manual devices was acceptable.

Conclusion: BP monitors evaluated demonstrated poor reliability which is in conflict with previous studies done. Further studies are needed to determine accuracy of these devices, especially those which are used in many clinical settings.

Key words: Accuracy, blood, pressure, devices

\section{CS/TH-O-4: HYPERLIPIDEMIA IN HIV- INFECTED PATIENTS ON LOPINAVIR/ RITONAVIR MONOTHERAPY IN RESOURCE- LIMITED SETTINGS}

Mitch Matoga' ${ }^{1}$, Mina Hosseininpour ${ }^{2}$, Avgenia Aga ${ }^{3}$, Heather Ribaudo ${ }^{3}$, Nagalingeswaran Kumarasamy ${ }^{4}$, John Barlett ${ }^{5}$, Michael Hughes ${ }^{3}$

'School of Public Health, Faculty of Health Sciences, University of the Witwatersrand, Johannesburg, South Africa, ${ }^{2}$ University of North Carolina Project, ${ }^{3}$ Harvard T.H. Chan School of Public Health, ${ }^{4}$ YRG Centre for AIDS Research and Education, ${ }^{5}$ Department of Medicine, Duke University Medical Centre
Cardiovascular disease (CVD) is an emerging concern for HIV-infected patients. Hyperlipidaemia is a risk factor for CVD and a complication of protease-inhibitorbased antiretroviral therapy, but little is known about its incidence and risk factors in treated patients in resourcelimited settings (RLS). We conducted a secondary analysis of ACTG-A5230 trial in which HIV-infected adults from India, Malawi, Tanzania, Thailand and South Africa, with virologic relapse on first line therapy were initiated on lopinavir/ritonavir (LPV/r) monotherapy. Hyperlipidaemia was elevated fasting total cholesterol (FTC $\geq 240 \mathrm{mg} / \mathrm{dll}$ ) or fasting triglycerides (FTG $2500 \mathrm{mg} / \mathrm{dll}$ ) or calculated low density lipoprotein cholesterol (LDL $\geq 160 \mathrm{mg} / \mathrm{dll}$ ) based on measurements at weeks $12,24,48,68$ and 104 after commencement of treatment. We evaluated factors potentially associated with quantitative lipid changes from baseline to week 12. These were age, sex, race, site, and baseline body mass index, CD4 cell count, HIV1 RNA level, and lipids. One hundred and six participants without hyperlipidaemia at baseline started LPV/r; median age 39 years, $68 \%$ black African, $55 \%$ female. Cumulative incidence at week 104 was $48 \%$ (95\% Cl: $36-58 \%)$. At week 12, there were significant mean increases from baseline in FTC (17 mg/dL, P<0.001) and FTG (104 mg/ $\mathrm{dL}, \mathrm{p}<0.001)$. In multivariable analysis, higher baseline FTC $(p=0.044)$, FTG $(p=0.025)$, Thai $(p<0.001)$ or Indian sites $(p=0.020)$ versus African sites were associated with increased risk of hyperlipidemia. In HIV-infected adults in RLS initiating LPV/r, hyperlipidaemia was common. Baseline lipid measurements and routine monitoring should be recommended in individuals starting LPV/rbased treatments with borderline high lipids.

Key words: Hyperlipidemia, HIV, Lopinavir/ritonavir

\section{CS/TH-O-5: CYTOKINES AS BIOMARKERS OF PANCREATIC DUCTAL ADENOCARCINOMA: A SYSTEMATIC REVIEW}

Yandiswa Yako',2, Deirdre Kruger', Martin Smith', Martin Brand

'Department of Surgery, School of Clinical Medicine, Faculty of Health Sciences, University of the Witwatersrand, Johannesburg, South Africa, ${ }^{2}$ Pancreatic Research Trust

Biomarkers for disease management in non-pancreatic cancers are well established, but not in pancreatic ductal adenocarcinoma (PDAC). Cytokines are emerging as potential biomarkers for PDAC due to their altered circulating levels in affected patients. A systematic review of the role of cytokines in clinical medicine as diagnostic, predictive, or prognostic biomarkers in PDAC was undertaken. Asystematicreviewwasconductedaccording to the 2009 PRISMA guidelines. PubMed database was searched for original articles on the topic of interest published until June 2015, and this was supplemented with references in relevant article. Studies were evaluated 
for risk of bias using the Quality in Prognosis Studies tools. Forty one cytokines were investigated in relation to PDAC in 65 studies, eleven of which were analysed by three or more studies. Six cytokines (IL-1 $\beta,-6,-8,-10$, VEGF, and TGF) were consistently reported to be increased in PDAC by more than four studies; irrespective of sample type, method of measurement or statistical analysis model used. Furthermore, the above-mentioned cytokines were correlated with poor prognosis and tumour progression. IL-1 $\beta,-6$ and -8 when evaluated as part of a panel that included CA19-9 were more sensitive and specific than CA19-9 alone in differentiating PDAC from benign pancreatic diseases. Our review demonstrated that there is insufficient evidence to support the role of individual cytokines as diagnostic, predictive or prognostic biomarkers for PDAC. However, emerging evidence indicates that a panel of cytokines may be a better tool for discriminating PDAC from other non-malignant pancreatic diseases.

Key words: Pancreatic ductal adenocarcinoma, cytokines, biomarkers

\section{CS/TH-O-6: CLINICAL, ANGIOGRAPHIC AND PROCEDURAL FACTORS ASSOCIATED WITH PERIPROCEDURAL MYOCARDIAL INFARCTION DURING PERCUTANEOUS CORONARY INTERVENTION}

Nqoba Tsabedze, Keir McCutcheon, Lancelot Mkhwanazi, Ahmed Vachiat, Rohan Ramje, Jameel Moosa, Themba Maluleke, Gloria Mukeshimana, Pravin Manga

Department of Internal Medicine/Cardiology, School of Clinical Medicine, Faculty of Health Sciences, University of the Witwatersrand, Johannesburg, South Africa

Percutaneous coronary intervention (PCI) is effective therapy for significant atherosclerotic coronary artery disease. Despite medical and technological advances in $\mathrm{PCl}$, periprocedural myocardial infarction (PMI) remains a common complication. The frequency and factors associated with PMI have been well investigated in the developed world yet there is a paucity of data from developing countries, especially in Sub-Saharan Africa. We prospectively enrolled 153 adult patients undergoing $\mathrm{PCl}$ at the Charlotte Maxeke Johannesburg Academic Hospital from the 1st of February 2014 to 31st October 2014. Periprocedural Creatinine Kinase-MB and hs-Troponin I were routinely measured before $\mathrm{PCl}$ and at $16-24$ hours post-procedure. The third universal definition of myocardial infarction was used to define a PMl event. One hundred and fifty two participants met the inclusion criteria and were analysed for PMI. There were $70.4 \%$ male participants. The mean age was 58.8 (SD 10.9) years. Sixteen (10.5\%) participants fulfilled the criteria for PMI. Side branch pinching with preserved TIMI III flow was noted in $62.5 \%$ of PMl cases. Duration of procedure $(p=0.007)$, right coronary artery intervention $(p=0.042)$ and total stent length $(p=0.045)$ were independently associated with PMI. PMl occurred in $10.5 \%$ of cases undergoing $\mathrm{PCl}$. This is consistent with the prevalence of PMI internationally. Duration of procedure was the most significant factor associated with PMI. Larger multicentre studies are required in our demographic region to further define relevant predictors and outcomes associated with PMI.

Key words: Periprocedural, Myocardial, Infarction, Percutaneous, Coronary Intervention

\section{CS/TH-0-7: DEHISCENCES AND FENESTRATIONS IN SOUTH AFRICAN SKULLS: PREVALENCE AND CHARACTERISATION}

Sandra Koutras, Govindrau Mohangi

Department of Oral Medicine and Periodontology, School of Oral Health Sciences, Faculty of Health Sciences, University of the Witwatersrand, Johannesburg, South Africa

The inadvertent exposure of alveolar defects during periodontal and oral surgery may result in complications such as recession with subsequent sensitivity and lack of osseointegration in the case of implants. The objective of this study was to determine the prevalence, distribution and characteristics of dehiscences and fenestrations in skulls of a South African population. A sample of 333 skulls from the Raymond A. Dart Collection of Human skeletons in the School of Anatomical Sciences, University of the Witwatersrand, Johannesburg, from 1980 to 2014 were examined. The size and distribution of these defects was assessed. The position of the affected teeth in the arch and the size of these teeth in relation to the width of the jaw were also recorded. A total of 770 alveolar defects were recorded. Of those, 579 were fenestrations with $63.2 \%$ in the maxilla and $36.7 \%$ in the mandible, whilst 191 were dehiscences with $72.8 \%$ in the mandible and $27.2 \%$ in the maxilla. The total number of defects in the maxilla (418) exceeded that of the mandible (352). In the maxilla, the teeth most commonly associated with both dehiscences and fenestrations were the first molars (39.2\%) and canines (29.9\%). In the mandible, the canines (34.9\%) and the first premolars $(28.8 \%)$ were the most commonly affected. There was a higher proportion of fenestrations compared to dehiscences in all teeth except the canines where dehiscences predominated. Of the 6010 teeth examined, $12.8 \%$ were associated with alveolar defects; positively correlating with the number of teeth present. Gender, age and population had no influence on the defects observed. Enhanced information surrounding these defects will provide clinicians insight on improved treatment options.

Key words: Alveolar defect, fenestration, dehiscence, Raymond A. Dart 


\section{CS/TH-O-8: UNRAVELLING RIGHT VENTRICULAR DYSFUNCTION IN ISOLATED LEFT VENTRICULAR NON-COMPACTION}

Samantha Nel', Ferande Peters', Elena Libhabher', Mohammed R. Essop' ${ }^{1}$ Claudia Dos Santos², Hiral Matioda², Ruchika Meel ${ }^{3}$, Nirthi Maharaj ${ }^{3}$

'School of Clinical Medicine, Faculty of Health Sciences University of the Witwatersrand, Johannesburg, South Africa,

${ }^{2}$ Cardiac Technologist, ${ }^{3}$ Cardiologist

The prevalence of right ventricular (RV) dysfunction varies among patients with isolated left ventricular noncompaction (ILVNC) and has not been systematically studied in a sub Saharan population. We aimed to 1) determine the prevalence of RV dysfunction in a sub Saharan population of ILVNC patients; 2) To compare the clinical and echocardiographic characteristics of ILVNC patients with RV dysfunction compared to those without RV dysfunction. Forty-nine patients with ILVNC, were enrolled in this cross-sectional study at Chris Hani Baragwanath Academic Hospital, and assessed for RV dysfunction using echocardiography. RV systolic function was evaluated using the tricuspid annular peak systolic velocity (S'). Patients with an RV S' $<10 \mathrm{~cm} / \mathrm{s}$ were classified as having RV systolic dysfunction.Statistically significant differences were evident in the ILVNC patients with RV dysfunction with regards to the left ventricular remodelling parameters, evident by the larger LVEDD (62.4 \pm 9.55 , $p=0.004)$, and increased sphericity indices (1.3 \pm 0.2 and $1.1 \pm 0.1, p=0.007$ and $p=0.002$ respectively). There were differences in the diastolic parameters seen in $\mathrm{E} / \mathrm{E}$ ' for the lateral wall $(16.5 \pm 10.9, p=0.01)$, reflecting the increase in $\mathrm{LV}$ filling pressures. There were differences in pulmonary hypertension, reflected in the elevated PAP $(48.4 \pm 22.3$, $\mathrm{p}=0.03$ ). In conclusion, the percentage of RV dysfunction in this study was almost $50 \%$. Patients with ILVNC and RV dysfunction had more adverse ventricular remodelling, increased filling pressures, increased MR and evidence of pulmonary hypertension, when compared to ILVNC with preserved RV function.

Key words: Left ventricular noncompaction, right ventricular function

\section{CS/TH-0-9: INDUSTRIAL AUDIOMETRIC SCREENING REFINED}

Angela Butkovic, Agnes Huiskamp

Department of Nursing Education, School of Therapeutic Sciences, Faculty of Health Sciences, University of the Witwatersrand, Johannesburg, South Africa

Audiometric surveillance and hearing conservation programmes have continued to show fragmentation and poor quality records in the United States of America, United Kingdom and South Africa. It was of great concern that the literature revealed a noticeable decline of sustained skill in audiometric screening, as predominantly conducted by the Audiometrist and/or Occupational Health Nurse Practitioner. The purpose of this study was to develop and test a refresher programme in occupational audiometry that could improve and sustain audiometric screening skills, improve the quality of audiogram records and enhance audiometric surveillance. Mixed methods were used within a pre-test, post-test intervention design. Qualitative data from the quota sampled hearing health specialists during semi-structured interviews was triangulated with the quantitative data from convenience sampled Audiometrists who completed the self-administered questionnaires. These findings were used to develop an appropriate and contemporary refresher programme aligned to the South African context. The intervention has been pilot tested in Gauteng where descriptive analysis revealed that the Quality Audiometric Record Checklist (QARC) designed to score the quality of audiograms proved to have a 0.9 average content validity index and a $>80 \%$ Kappa-Fleisch inter-rater agreement. Participants indicated that they wanted a refresher programme (94\%) using blended learning approach (63\%) and/or traditional approach (29\%). Although the intervention has revealed a clinical and statistical improvement in a short term impact analysis, further testing is recommended before national implementation of the programme can occur in collaboration with the South African Society of Occupational Health Nursing.

Key words: Quality, Audiometry, Intervention

\section{CS/TH-O-10: BIOCHEMICAL MARKERS OF MINERAL BONE DISORDER IN PATIENT ON MAINTENANCE HAEMODIALYSIS}

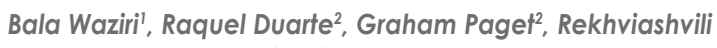
Vakhtang $^{3}$, Saraladevi Naicer ${ }^{2}$

'School of Clinical Medicine, Faculty of Health Sciences, University of the Witwatersrand, Johannesburg, South Africa, ${ }^{2}$ Department of Internal Medicine, Faculty of Health Sciences, University of the Witwatersrand, Johannesburg, South Africa; ${ }^{3}$ Renal Unit, Donald Gordon Medical Centre, University of the Witwatersrand, Johannesburg, South Africa

Despite the high mortality and morbidity associated with abnormalities in mineral and bone metabolism in haemodialysis patients, there are limited data on the pattern of mineral bone disorder in African CKD populations. Therefore, the purpose of this study was to describe the pattern of mineral bone disease by evaluating biochemical parameters in patients on maintenance haemodialysis (MHD). We evaluated the serum/plasma intact parathyroid hormone (iPTH), calcium, phosphate, alkaline phosphatase (ALP) and $25-\mathrm{OH}$ vitamin $\mathrm{D}$ levels of two hundred and five patients undergoing MHD at two dialysis centres in Johannesburg. The MHD patients (133 men, 72 women) had a mean age of $54.2 \pm 15.3$ years with a median dialysis vintage of 
24 months (IQR, 12-48) and a mean $\mathrm{kt} / \mathrm{v}$ of $1.4 \pm 0.3$. The prevalence of hyperparathyroidism (iPTH $>150 \mathrm{pg} / \mathrm{mL}$ ), hyperphosphataemia, hypocalcaemia and $25-\mathrm{OH}$ vitamin D deficiency $(<30 \mathrm{ng} / \mathrm{mL})$ was $72.1 \%, 54.1 \%, 20.4 \%$ and $80.1 \%$ respectively. There was a positive correlation between IPTH and ALP ( $r=0.22, \quad P=0.007)$. The combination of markers of bone turnover (iPTH $>150$ and ALP> $110 \mathrm{U} / \mathrm{L}$ ) suggestive of high turnover bone disease, was present in $46.8 \%$ of the patients. The odds ratio for developing hyperparathyroidism with hypocalcaemia and hyperphosphataemia were $4.7195 \%$ $\mathrm{Cl} 1.0-22.3, \mathrm{p}=0.04)$ and $2.9(95 \% \mathrm{Cl} 1.4-6.4, \mathrm{p}=0.005)$ respectively. Fouty eight percent of MHD patients had $\mathrm{PTH}$ within the recommended KDIGO guidelines. Secondary hyperparathyroidism and $25-\mathrm{OH}$ vitamin $D$ deficiencies were common in our haemodialysis patients. Hypocalcaemia and hyperphosphataemia were strong predictors for developing secondary hyperparathyroidism

Key words: Secondary hyperparathyroidism, 25-OH vitamin $D$, haemodialysis

\section{CS/TH-O-11: RENAL AND HEPATITIS SCREENING FOR ELIGIBILITY FOR ANTIRETROVIRAL TREATMENT IN SOUTH AFRICA: PUBLIC HEALTH LESSONS FROM AN URBAN CLINICAL TRIAL}

Michelle Moorhouse

School of Clinical Medicine, Wits Reproductive Health and HIV Institute (WRHI)

Current recommended first-line ART in SA is tenofovir, emtricitabine or lamivudine with efavirenz. Screening for renal, hepatic and other disorders, as well as toxicity monitoring complicates first-line ART. An uncommon but potentially catastrophic side-effect of tenofovir is renal failure. Recommendations to decrease tenofovir toxicity include creatinine clearance assessment prior to ART initiation, with subsequent monitoring. Tenofovir with lamivudine/emtricitabine is a potent suppressor of hepatitis B (HBV). HBV screening is advisable prior to tenofovir initiation, as it may predict for immune reconstitution syndromes, and because unexpected interruption of suppressive tenofovir and lamivudine/ emtricitabine may cause severe hepatitis flares. Does pre-ART screening add to patient management? Seven hundred and seventy one HIV-positive antiretroviralnegative adults from inner-city primary healthcare clinics and an academic hospital in Johannesburg were recruited for a clinical trial. Participants had to be eligible for ART, based on CD4 count (350cells/mm3). Laboratory screening was done for anaemia, HBV-surface antigen (HBsAg) and renal dysfunction. Referral CD4 cell counts were repeated. No participants reported a history of renal dysfunction or HBV infection. HBV exposure was common and 59/771 (7.7\%) were HBsAg-positive. Only 4/771 (0.5\%) had severe anaemia (haemoglobin $<7 \mathrm{~g} / \mathrm{dL}$ ). Creatinine clearance rates were almost all normal 766/771 (99\%), although 171/771 (22\%) had microalbuminuria. HBV is a common co-infection in HIV-infected adults, and adds complexity to managing ART switches involving tenofovir. Routine renal and haemoglobin screening prior to ART appeared to add little to patient management, but may result in increased cost and delays in ART initiation

Key words: Tenofovir, renal, hepatitis B

\section{CS/TH-O-12: THE VALUE OF NEUTROPHIL LYMPHOCYTE RATIO IN THE DIAGNOSIS OF ACUTE APPENDICITIS}

Peter Asafo-Adjei, Thifhelimbilu Luvhengo, Geoffrey Candy

Department of Surgery, School of Clinical Medicine, Faculty of Health Sciences, University of the Witwatersrand, Johannesburg, South Africa

White cell count (WCC) and C-reactive protein (CRP) are of limited value in the early detection of acute appendicitis (AA). The neutrophil lymphocyte ratio (NLR) has been proposed as a novel cost effective marker that could play this role. The aim of the study was to evaluate reliability of NLR in the diagnosis of acute appendicitis in the African setting. A retrospective review of records of patients treated for AA from January 2003 to June 2015 was conducted. Data retrieved included patients' demography, pre-operative WCC with differential count, CRP and histological findings. NLR was calculated for each patient. ROC curves were used to determine cut-off value for each test. Sensitivity, specificity, PPV and NPV were also calculated. A total 1098 records were found of which 590 were excluded. Average age of patients was 28 years and $56.7 \%$ were males. The cut-off NLR value for the diagnosis of AA was 2.55 with a PPV and NPV sensitivity and specificity, of $81.1 \%, 47.5 \%, 94.4 \%$ and $18.8 \%$; respectively. WCC of 9.16 and CRP of 13 were diagnostic of AA. WCC showed sensitivity of $67.9 \%$, specificity of $55.0 \%$, PPV of $94.4 \%$, NPV of $13.7 \%$ for WCC. CRP had sensitivity of $89.8 \%$, specificity of $42.5 \%$, PPV of $94.4 \%$ and NPV of $27.9 \%$. A cut-off NLR of 4.94 differentiated complicated from uncomplicated AA while 10.09 was diagnostic of serosal AA. Area under the curve (AUC) for NLR, WCC and CRP were $0.662,0.622$ and 0.687 , respectively. Although NLR is less sensitive compared to CRP it is more specific for diagnosis of AA. A cut off of 4.90 differentiates complicated from uncomplicated AA.

Key words: Acute appendicitis, neutrophil lymphocyte ratio, complicated appendicitis 


\section{CS/TH-O-13: COMPUTER AIDED DIAGNOSIS FOR WHO CHEST X-RAY PRIMARY END-POINT PNEUMONIA IN CHILDREN}

Nasreen Mahomed, Bram Van Ginneken, Rick Philipsen, Jamie Melendez, David Moore, Tanusha Sewchuran, Halvani Moodley, Shabir A Madhi

Department of Diagnostic Radiology, School of Clinical Medicine, Respiratory and Meningeal Pathogens Research Unit, Faculty of Health Sciences, University of the Witwatersrand, Johannesburg, South Africa

The chest $X$-ray remains the commonest imaging modality for the assessment of childhood pneumonia. World health organisation (WHO) standardised chest X-ray interpretation is important. Objective: To determine the sensitivity and specificity of computer aided diagnosis (CAD) software (CAD4WHOKids) for WHO chest X-ray primary end-point pneumonia (CXR-PEP) compared to consensus human interpretation. This study is nested within the PERCH study, South African site. Chest $X$-rays were independently read by three radiologists using modified WHO standardised chest $\mathrm{X}$-ray interpretation criteria and majority consensus was used. CAD4WHOKids involved automatic lung field segmentation followed by manual inspection and correction. Areas of WHO CXR-PEP and areas of other infiltrate were manually drawn to train CAD4WHOKids using a texture analysis system. Pixels in outlined regions were used as positive examples. Training and testing was done in 10-fold cross validation. Pixel data was filtered with Gaussian derivatives on multiple scales, extracting texture features to classify each region. To obtain an image score, the 95th percentile score of the pixels was used. For WHO CXR-PEP versus non WHO CXR-PEP, using the 858 interpretable chest $X$-rays, CAD4WHOKids had a sensitivity of $76 \%$, specificity of $80 \%$ and area under the ROC curve of $0.850(95 \% \mathrm{Cl} 0.823-0.876)$ using the radiologist consensus reading as the gold standard. For WHO CXR-PEP versus other infiltrate only; where normal chest X-rays were excluded from the 541 chest $X$-rays, CAD had a sensitivity of $77 \%$, specificity of $73 \%$, and area under the ROC curve of $0.810(95 \% \mathrm{Cl} 0.772-0.846)$. The results of CAD4WHOKids are promising for identifying WHO CXR-PEP in children.

Key words: Chest X-rays, computer aided diagnosis

\section{CS/TH-O-14: CORRELATES OF CAROTID INTIMA MEDIA THICKNESS IN CHILDREN WITH CHRONIC KIDNEY DISEASE}

Abdullahi Mudi', Zaiboonisa Holland², Caroline Dickens², Daynia Ballot $^{3}$, Cecil Levy ${ }^{3}$

'School of Clinical Medicine, Department of Paediatrics, Faculty of Health Sciences, University of the Witwatersrand, Johannesburg, South Africa, ${ }^{2}$ Charlotte Maxeke Johannesburg Academic Hospital, ${ }^{3}$ University of the Witwatersrand

Cardiovascular disease (CVD) is a common complication of Chronic Kidney Disease (CKD). In children with CKD, CVD may manifest as early changes in arterial thickness and or stiffness. We aimed to determine clinical and biochemical correlates of Carotid Intima Media Thickness (cIMT) in children with CKD. Thirty-four children with CKD were recruited into the study. Each had a physical examination and routine follow up blood tests. Carotid Doppler ultrasound and 2D echocardiography were carried out on each of the patients to determine the average CIMT and the left ventricular mass index (LVMI). The mean age of the children was $12.1 y \pm 3.4$, with $20 / 34$ males and $14 / 34$ females and a mean cIMT of $0.512 \mathrm{~mm} \pm 0.066$. There were 15/34 pre-dialysis CKD patients and 19/34 on chronic dialysis. The mean CIMT was greater in patients on dialysis $(0.539 \mathrm{~mm} \pm 0.014)$ when compared to the pre-dialysis CKD patients $(0.478 \mathrm{~mm} \pm 0.015)$, and there was a significant difference between the two means $(p=0.006)$. There was a significant positive correlation of CIMT with duration on dialysis, blood urea, alkaline phosphatase and PTH levels $(p<0.05)$. LVMI also had a positive correlation with cIMT $(p=0.015)$. There was a significant negative correlation of CIMT with eGFR and haemoglobin levels $(p<0.05)$. Various clinical and biochemical correlates of CIMT may be used as predictors of early cardiovascular risk. A larger study looking at these correlates when adjusted for using a regression model may provide more information on their individual role in predicting early cardiovascular risk.

Key words: CIMTs, Children, CKD

\section{CS/TH-O-15: EXPLORING THE CONSEQUENCES OF CASH TRANSFERS FOR ADOLESCENT BOYS AND GIRLS IN INNER-CITY JOHANNESBURG}

Nomhle Khoza', Jonathan Stadler ${ }^{1}$, Catherine MacPhail', Admire Chikawanda', Heena Brahmbhatt ${ }^{3}$, Sinead Delany-Moretlwe' 'Wits Reproductive Health and HIV Institute, Faculty of Health Sciences, University of the Witwatersrand, Johannesburg, South Africa, '2University of New England, ${ }^{3} J o h n$ Hopkins University

Cash transfers (CTs) are increasingly being explored as a structural approach for HIV prevention. While there is much interest in expanding CT programmes to leverage health-related outcomes, there are few available data on the potential social consequences of offering CTs to adolescents. This paper explores the consequences of CTs on adolescent recipients. Using qualitative data collected during a pilot randomised controlled trial of three cash transfer strategies conducted in 120 consenting adolescents aged $\geq 16$ years in the inner-city of Johannesburg. In-depth interviews were conducted with a sub-sample of 41 participants six months after receiving the CT and up to 12 months after the cash was withdrawn. Codes were generated using an inductive approach. Initial transcripts were coded based on emerging issues and subsequently transcripts were coded deductively. Atlas-ti 7.5 was used to organise and code data. Overall, 
CTs were highly acceptable to recipients. They were used for personal items and reduced household stress. This was interpreted by recipients as a sign of maturity and independence. Boys' spending and saving patterns reflected a concern with maintaining their public social status, through which they asserted an image of the responsible adult. In contrast, girls' spending and saving reflected domestic concerns. Although generally regarded positively, adolescents reported some negative consequences of CTs. These data suggest that CTs benefit individuals and households in dense, urban environments, and may instil responsibility in young adults. However, negative consequences of CTs need to be monitored and interventions that address alcohol and drug use could be included in CT programmes.

Key words: Cash transfers, adolescents, gender, HIV prevention

\section{CS/TH-O-16: PREVALENCE OF FACTORS INFLUENCING RECURRENCE AND/OR METASTASIS IN DERMATOFIBROSARCOMA PROTUBERANS}

Joshua Kiluba', Benneh AY', Kay PS', Hale MJ², Luvhengo TE'

'Department of Surgery, School of Clinical Medicine, Faculty of Health Sciences, University of the Witwatersrand, Johannesburg, South Africa, ${ }^{2}$ Department of Anatomical Pathology, Faculty of Health Sciences, University of the Witwatersrand, Johannesburg, South Africa

Dermatofibrosarcoma protuberans (DFSP) has limited metastatic potential. The fibrosarcomatous variant of DFSP has increased propensity for local and distant recurrence. The aim of the study was to determine the prevalence of markers of aggressive DFSP. We undertook a retrospective review of records of patients with DFSPs. Data retrieved included patients' demography, tumour site, size, biopsy type, excision margin, CD34 expression, mitotic count, presence of necrosis and evidence of fibrosarcomatous change. Seventy five histopathological records were found, of which $25.3 \%$ were recurrent. All were from black patients and majority $43 / 75$ (57.3\%) were females. Their average age was 39.7 years. Majority (66.7\%) were in the trunk and the average size was $8.1 \mathrm{~cm}$ (range: $1.5 \mathrm{~cm}-19.5 \mathrm{~cm})$. Excision was performed in $43 / 75$ (57.3\%) and was deemed wide local excision in 35/43. Resection margin was adequate in $5.7 \%$. FS-DFSP was reported in $12 / 75(16.0 \%)$ overall and $12 / 43(27.9 \%)$ of excised DFSPs. Most $9 / 12$ (75.0\%) of FS-DFSPs were in females. Only $9.7 \%$ of classical DFSPs involved limbs whereas $3 / 12$ (25.0\%) of FS-DFSPs were in extremities. Average age of patients and size of FS-DFSPs were 44.7 years and $10.4 \mathrm{~cm}$, respectively. Mitotic count of five and above per 10 high-power fields was reported in $74.9 \%$ (9/12) of FS-DFSPs. DFSP is a disease of young adults and majority are above $5 \mathrm{~cm}$ at presentation. FS-DFSP variant of DFSP affects older patients, is larger and likely to have mitotic count above 10 per 10 per high power field. Adequate tumour resection margin is rarely achieved.

Key words: Dermatofibrosarcoma protuberans, fibrosarcomatous variant, recurrence, metastasis

\section{CS/TH-0-17: THE FUNCTIONAL ABILITY AND HEALTH RELATED QUALITY OF LIFE OF SURVIVORS OF CRITICAL ILLNESS AFTER HOSPITAL DISCHARGE}

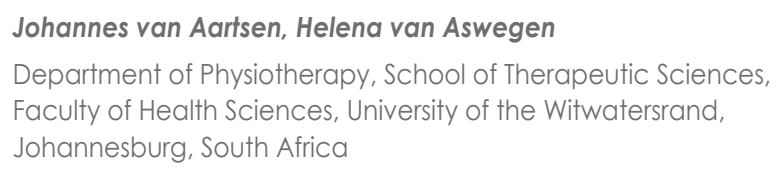

Critically ill patients face physical, psychological, and health-related quality of life (HRQOL) problems. Limited South African data is available regarding the HRQOL of survivors of critical illness and limited research evidence is available to support rehabilitation services for this population after hospital discharge. The aim was to assess functional ability and HRQoL of survivors of critical illness over the first six months after hospital discharge using a prospective, observational, longitudinal design. Severity of illness, mechanical ventilation (MV), ICU and hospital duration and return to work were recorded. Physical function (PFIT-s) was assessed at ICU and hospital discharge. Peripheral muscle strength, exercise endurance (6MWT), HRQOL (SF-36 and EQ-5D questionnaires) and level of depression (PHQ-9 questionnaire) were measured at one and six months after discharge. Twenty-four participants were enrolled (drop-out rate: $n=9,37.5 \%$ ). Mean age was 51 years ( $\pm S D$ 13.8). Three participants $(20 \%)$ returned to work at one month and six $(54.5 \%)$ at six months after discharge. PFIT-s scores changed significantly between ICU and hospital discharge $(p=0.02)$. Changes in mean 6MWT distance achieved were not significant. Changes observed in mean peripheral muscle strength were significant at six months. Significant changes were found in mean SF-36 PCS $(p=0.00)$, role physical $(p=0.00)$, bodily pain $(p=0.05)$, general health $(p=0.00)$, vitality $(p=0.01)$ and social functioning $(p=0.00)$ scores, and mean EQ-5D visual analogue scale (VAS) scores $(p=0.00)$ at six months. $P H Q-9$ scores had a positive association ( $r=0.65)$ with mental health status at one $(p=0.04)$ and six months $(p=0.00)$. Duration of $M V$ and hospital stay had a negative association with physical component score (PCS) at six months ( $r=-0.69 ; p=0.02)$. Limitations included small sample size and high drop-out. Survivors of critical illness experienced improvements in functional abilities and QoL, but continued to experience limitations in exercise endurance and QoL after discharge from hospital.

Key words: Critical illness, QOL 


\section{CS/TH-0-18: A NOVEL PEPTIDE- ENHANCED ENDOSTATIN-LOADED NANO-CONSTRUCT FOR TARGETED ANTI- ANGIOGENIC EFFECT IN SQUAMOUS CELL CARCINOMA}

Samson Adeyemi', 2, Viness Pillay², Yahya Choonara², Lisa du Toit $^{2}$, Pradeep Kumar ${ }^{2}$

'Department of Pharmacy, School of Therapeutic Sciences, Faculty of Health Sciences, University of the Witwatersrand, Johannesburg, South Africa, ${ }^{2}$ Wits Advance Drug Delivery Platform (WADDP) Research Unit

Targeted delivery of smart nanosystems is a prospective alternative to circumvent the draw backs encountered with many conventional cancer chemotherapeutics with non-specific targeting, sub-optimal dosage and adverse effects on healthy cells. Short peptides could be employed to functionalise the surface of novel nano constructs since cancer cells over-express certain proteins on their cellular membrane as opposed to normal cells. This study aimed to design and characterise novel pentapeptide-enhanced nanoparticles as targeted delivery cargo for the delivery of endostatin in Squamous Cell Carcinoma (SCC) management. Endostatin-loaded nanoparticles were successfully synthesised using an ionic gelation procedure and characterised for structural and functional modifications, particle size, morphology, zeta potential, drug loading efficiency and release kinetics. Surface modification of the synthesized endostatinloaded nanoparticles was achieved using CGKRK as a homing peptide. The cytotoxic effects of the CGKRK functionalised endostatin-loaded nanoparticles were examined using MTT assay on an established SCC cell line (KYSE-30). HI NMR and FT-IR experiments showed that the native polymers were successfully grafted. The average size and morphology of the endostatin-loaded nanoparticles as confirmed by TEM, and SEM results showed that the nanoparticles exhibited a spherical shape and had a narrow particle size distribution with polydispersity index (PDI) less than 0.5 with an average size below $100 \mathrm{~nm}$. A positive value zeta potential of $17.3 \mathrm{mV}$ was obtained for the nanoparticles. The drug release kinetics was optimal at $\mathrm{pH} 6.8$ than at the physiological $\mathrm{pH}$. The cell proliferation assay showed that the peptideenhanced endostatin-loaded nano constructs exhibited increased cytotoxic effects on the KYSE-30 cell lines than the non-targeted endostatin loaded nanoparticles.

Key words: Targeted delivery, Smart Nanosytems, Homing Peptides, Squamous Cell Carcinoma

\section{CS/TH-P-1: HIGH RATE OF ABNORMAL LEFT VENTRICULAR MASS INDEX AMONG PAEDIATRIC DIALYSIS PATIENTS}

Abdullahi Mudi, Caroline Dickens, Daynia Ballot, Cecil Levy

Department of Paediatrics, School of Clinical Medicine, Faculty of Health Sciences, University of the Witwatersrand, Johannesburg, South Africa
Left ventricular (LV) changes are common in children on chronic dialysis. Increase in LVMI has been associated with poor blood pressure control and may lead to poor systolic function. In our clinic, cardiovascular problems are the main reason for exclusion from the transplant list. We aimed to determine the rate of abnormal LVMI in our dialysis patients. Twenty seven children (5 to 18 years) on chronic dialysis had a general examination, blood pressure and 2D echocardiography performed. The left ventricular end diastolic dimension (LVEDD), intraventricular septum thickness at end diastole (IVSd), the left ventricular posterior wall thickness at end diastole (LVPWd) and the left ventricular ejection fraction (EF) was determined for each child. The left ventricular geometry of the heart was determined from the RWT and classified into normal, concentric remodelling (CR), concentric hypertrophy $(\mathrm{CH})$ and eccentric hypertrophy (EH). Mean age 12.2 years \pm 3.8 ; male: female ratio $1.45: 1 ;$ HD 20/27; PD 7/27; median duration of dialysis 15 months (5-50). Twenty one of the 27 children had hypertension; 18/27 had an abnormal LVMI of which 10/18 had a severe abnormality. Fourteen of the 27 children had $\mathrm{CH}, 4 / 27$ had $\mathrm{EH}$ and 4/27 had CR. Four of the 27 children had a low EF (<45\%). For every unit increase in mean arterial pressure (MAP) beyond $80 \mathrm{mmHg}$, LVMI increased by $0.5 \mathrm{~g} / \mathrm{m} 2$ ( $p=0.009)$. A high rate of LVMI was observed in our cohort. Only MAP had a significant correlation with LVMI. We recommend the use of MAP for the assessment of blood pressure control and predicting risk for abnormal LVMI.

Key words: Abnormal, LVMI, Paediatrics, Dialysis

\section{CS/TH-P-2: DISABILITY IN PEOPLE LIVING WITH HUMAN IMMUNODEFICIENCY VIRUS IN SOUTH AFRICA, CANADA AND IRELAND}

Adetunji Adeleke, Denise Franzsen, Rulaine Smith Department of Occupational Therapy, School of Therapeutic Sciences, Faculty of Health Sciences, University of the Witwatersrand, Johannesburg, South Africa

Disability in people living with Human Immunodeficiency Virus (HIV) has become a major health problem ranging from impairments in body functions to limitations in activity participation and restriction in social role inclusions. The severity of disability in people living with HIV in South Africa, Canada and Ireland is compared on the HIV Disability Questionnaire and the ICF codes for HIV for 80 South African, 136 Canadian and 96 people living with HIV in Ireland. Of the South African sample 23 were males while 57 were females. Common impairments reported were associated with endocrine, digestive and metabolic function (83.7\%), sensory function (83.5\%), haematological, immunological and respiratory function (82.5), activity limitations in area of mobility (56.4\%), major life areas (55.1\%) and social and civic life were also 
observed. Majority of the Canadian and Irish participants were men (82\% Canada and $74 \%$ Ireland) and $91 \%$ and $88 \%$ of them are on antiretroviral therapy in Canada and Ireland respectively. South African and Irish participants were younger than the Canadian participants. Irish participants reported living with lower median number of comorbidities. Disability has been revealed to be higher in South Africa followed by Canada and Ireland respectively. All health care providers need to understand how affected body systems and mind may ultimately influence an individual's activity and participations.

Key words: Disability, HIV, Limitation, Restriction

\section{CS/TH-P-3: LEVAMISOLE AFFECTS THE EXPRESSION OF TRANSCRIPTIONAL FACTORS SNAIL AND SLUG IN COLORECTAL CANCER CELLS}

Amber Khan, Kumar Subramanian, Jeyalakshmi Kandhavelu, Paul Ruff, Clement Penny

Medical Oncology Division, Department of Internal Medicine Faculty of Health Sciences, University of the Witwatersrand, Johannesburg, South Africa

Colorectal cancer is an important cause of mortality both globally and in South Africa. Once metastasized, with growth of the colorectal cancer cells in distant locations, such patients are often harder to treat and have a less favourable outcome. Metastatic dissemination involves the epithelial to mesenchymal transition (EMT) of tumour cells, with the cellular expression of EMT transcription factors and associated proteins, conferring aggressive migratory properties on the cells. Snail and Slug are two key EMT markers expressed in colorectal cancer cells. Here using confocal microscopy and quantitative polymerase chain reaction (GPCR) we evaluated the effects of the adjuvant drug levamisole, on the expression of Snail and Slug and on cell migration. Confocal microscopic analysis showed a considerable qualitative reduction in the expression of both the proteins Snail and Slug in DLD 1 (late stage) colorectal adenocarcinoma cells. However, cell migration was retarded in a dose dependent manner in HT29 (middle stage) and DLD 1 colorectal adenocarcinoma cells. After eight hours of $5 \mathrm{mM}$ levamisole treatment, cell shape was predominantly shrunken and rounded, cell growth was arrested and cell migration was inhibited to $102.2 \pm 0.9 \%$ in HT29 cells. GPCR analysis (in HT29) of the cells exposed to $5 \mathrm{mM}$ levamisole showed down regulation of EMT marker Snail. At a concentration of $5 \mathrm{mM}$, cells had fewer membrane protrusions and filopodia. Thus, therapies affecting the expression of Snail and Slug hold good promise to reduce the risk of EMT and dissemination of the cancer.

Key words: Levamisole, Snail, Slug, Colorectal cancer
CS/TH-P-4: REDUCED NON-DOMINANT LUMBAR MULTIFIDI CROSS-SECTIONAL AREA IS A PRECURSOR OF LOW BACK INJURY: A PROSPECTIVE COHORT STUDY

Benita Olivier, Nadia Gillion, Aimee Stewart, Warrick McKinon Department of Physiotherapy, School of Therapeutic Sciences, Faculty of Health Sciences, University of the Witwatersrand, Johannesburg, South Africa

The lumbarmultifidimuscle (LM) anticipates movement and stabilizes the lumbar spine prior to movement. Asymmetry in the LM cross-sectional area (CSA) is associated with low back injury. The aim of this prospective cohort study was to investigate the symmetry of the CSA of LM at L3, L4 and $\mathrm{L} 5$, in the context of simultaneous injury monitoring. Injury free, male, right-handed cricket fast bowlers playing at a non-professional level participated in this study. LM CSA at L3, L4 and L5 vertebral levels was measured through the use of ultrasound imaging in prone. The primary outcome measure of the study was the incidence of an injury during a cricket season of eight-month duration. Twenty-six fast bowlers (aged 21.8 \pm 1.8 years) participated. No difference was found between the left and the right LM CSA amongst injury free bowlers $(p>0.05)$. However, in bowlers who sustained a lower back injury during the cricket season, the left CSA at L3 (left median $5.80 \mathrm{~cm} 2$, range $3.69 \mathrm{~cm} 2$; right median $7.38 \mathrm{~cm} 2$, range $2.61 \mathrm{~cm} 2 ; \mathrm{p}=0.04$ ) and $\mathrm{L} 5$ (left median $6.94 \mathrm{~cm} 2$, range $2.10 \mathrm{~cm} 2$; right median $7.38 \mathrm{~cm} 2$, range $3.54 \mathrm{~cm} 2 ; p=0.04$ ) is smaller compared to the right. As none of the bowlers who sustained a lower back injury during the season had a previous lower back injury, these findings may indicate that the difference in LM CSA may be a precursor of injury.

Key words: Multifidi, cricket, fast bowler, ultrasound imaging

\section{CS/TH-P-5: NOVEL APPROACH TO THE DETECTION OF LEFT VENTRICULAR HYPERTROPHY USING BODY MASS INDEX- CORRECTED ELECTROCARDIOGRAPHIC VOLTAGE CRITERIA IN A GROUP OF AFRICAN ANCESTRY}

\author{
Chanel Robinson', Angela J Woodiwiss', Carlos D Libhaber', 2 , \\ Gavin R Norton' \\ 'School of Physiology, Cardiovascular Pathophysiology and \\ Genomics Research Unit, University of the Witwatersrand, ${ }^{2}$ School \\ of Clinical Medicine, Faculty of Health Sciences, University of the \\ Witwatersrand, Johannesburg, South Africa
}

Left ventricular hypertrophy (LVH) is a well-established endorgan change that enhances the ability to risk predict. Electrocardiographic (ECG) criteria for LVH detection are the most cost-effective method of detecting LVH. However, QRS voltages used to generate criteria for LVH detection are considerably attenuated by obesity. This effect renders the utility of ECG criteria to detect LVH in 
obese individual of African ancestry to be of limited value. We aimed to determine whether a novel approach to correcting $Q R S$ voltages for the attenuating effect of body mass index (BMI) will improve the ability of ECG criteria to detect LVH in a group of African descent. Left ventricular mass was determined from echocardiography in 661 randomly selected participants $(43.0 \%$ obese) of black African ancestry from the South West Township (SOWETO) of Gauteng. As compared to Cornell and Sokolow-Lyon voltage criteria, BMI best correlated with RaVL, GubnerUngerleider and Lewis QRS complex voltages but these relations were noted only in those with $\mathrm{BMI}$ below but not $\geq 29 \mathrm{~kg} / \mathrm{m} 2$. Correcting RaVL and Lewis voltages by the difference in the slope of BMl-voltage relations in those with $B M \mid<29 \mathrm{~kg} / \mathrm{m} 2$ versus those with $B M l \geq 29 \mathrm{~kg} / \mathrm{m} 2$ showed the greatest performance for $\mathrm{LVH}$ detection (uncorrected $\mathrm{RaVL}=0.695 \pm 0.025$, corrected $\mathrm{RaVL}=0.733 \pm 0.022$, $\mathrm{p}<0.0001$ ), and also increased the sensitivity (uncorrected $\mathrm{RaVL}=30.6 \%$ and corrected $\mathrm{RaVL}=42.4 \%, \mathrm{p}<0.0005$ ) with no significant change in specificity (uncorrected RaVL=86.3\% and corrected RaVL=83.0\%, $p=0.28$ ). In conclusion, we offer a novel approach to correcting ECG voltages for the attenuating effects of obesity in individuals of African ancestry and this improves the performance and sensitivity for LVH detection.

Key words: Left ventricular, hypertrophy electrocardiogram

\section{CS/TH-P-6: LEVELS OF CIRCULATING CYTOKINES IN SOUTH AFRICAN PANCREATIC DUCTAL ADENO- CARCINOMA PATIENTS: A PRELIMINARY REPORT}

Deirdre Kruger', Yandiswa Yako', Martin Brand ${ }^{1,2}$, John Devar', 2 Nicola Lahoud', Martin Smith ${ }^{1,2}$

'Department of Surgery, School of Clinical Medicine, Faculty of Health Sciences, University of the Witwatersrand, Johannesburg, South Africa, ${ }^{2}$ General Surgery, Chris Hani Baragwanath Academic Hospital

Several studies have investigated the association of differentially expressed cytokines with pancreatic ductal adenocarcinoma (PDAC), but none in African countries. In addition, US studies report considerably higher pancreatic cancer rates in Black patients than in any other racial group. This study aimed at investigating T-helper (Th) cell and angiogenic cytokines as diagnostic or prognostic biomarkers for PDAC in Black South Africans. We conducted a prospective, case-control study which included 34 PDAC patients and 27 control participants with either abdominal aortic aneurysm or acute abdomens from causes other than HPB. Plasma levels of IL-2, IL-4, IL-6, IL-10, TNF, IFN-gamma, IL-17A, VEGF, sVEGF-RI, FGF, PIGF, PDGF and P-selectin were measured using the commercially available Th1/Th2/Th17 BDTM cytometric bead array, single ELISA and multi-analyte Luminex kits. Significantly higher levels of IFN-gamma $(p<0.001)$, TNF $(p<0.001)$, IL-2 $(p=0.001), I L-4(p=<0.01), I L-10(p<0.01), I L-17 A$ $(p<0.01), P I G F(p<0.0001)$ and FGF-basic $(p<0.0001)$ were found in PDAC patients compared to control participants. A sub-group analysis of PDAC patients demonstrated that those with irresectable tumours had higher plasma levels of VEGF $(p=0.017)$ and IL-6 $(p=0.01)$. A univariate analysis showed significant associations between IFN-gamma, TNF, IL-10, -4, -2, FGFbasic, PIGF and PDAC. In a multivariate logistic regression model, FGF-basic (OR 38.9; $95 \% \mathrm{Cl}$, 3.6-415.3; $p<0.01$ ) and PIGF (OR 0.11; 95\% Cl, 0.02-0.56; $p<0.01$ ) were independent risk factors for PDAC. Our preliminary data suggests a potential role for FGF-basic, PIGF, IL-10, IFN-gamma and TNF as diagnostic biomarkers and VEGF and IL-6 as prognostic biomarkers of PDAC in Black South African patients.

Key words: Cytokines, biomarkers, pancreatic, adenocarcinoma

\section{CS/TH-P-7: CLINICAL CHARACTERISTICS OF HIV RELATED CARDIOMYOPATHY PATIENTS IN AN URBAN HOSPITAL IN SOUTH AFRICA}

D Zachariah, K McCutcheon, A Vachiat, N Tsabedze, R Ramjee, J Moosa, T Maluleke, G Muskesimana, M Mathenjwa, P Ewing, V Paton, P Manga

School of Clinical Medicine, Division of Cardiology - Charlotte Maxeke Johannesburg Academic Hospital, Faculty of Health Sciences, University of the Witwatersrand, Johannesburg, South Africa

HIV related cardiomyopathy (CMO) is one of the most common HIV related cardiac manifestations seen in sub-Saharan Africa. We recently reported a prevalence of $3.9 \%$ of HIV related CMO of all admissions to our unit. Thus the aim of this study was to ascertain the clinical characteristics of this cohort of patients. We analysed our clinical registry and found a total of 204 patients with HIV related $\mathrm{CMO}$. Of these, 156 patients had adequate data and thus comprised the current study cohort. The mean age was 38years (SD \pm 9 ) with 95 female patients $(61 \%)$ and 61 male patients. The mean CD4 count was $167 \mathrm{cell} / \mathrm{mm} 3$ (SD \pm 102 ). HIV related CMO patients generally did not have markedly enlarged left ventricular dimensions with the mean left ventricular dimension in diastole to be only $50 \mathrm{~mm}(S D \pm 4.1)$. The mean left ventricular ejection fraction was $39 \%$ (SD \pm 5.8 ). There was a high (38\%) incidence of anaemia as defined by WHO definition (males $<14 \mathrm{~g} / \mathrm{dl}$ \& females $<12 \mathrm{~g} / \mathrm{dl}$ ). The mean hemoglobin levels were found to be $9.2 \mathrm{~g} / \mathrm{dl}$ (SD \pm 3.9$)$. The mean estimated glomerular filtration rate (eGFR) was $104 \mathrm{ml} / \mathrm{min} / 1.73 \mathrm{~m} 2$ (SD \pm 22 ). The prevalence of low eGFR $(<60 \mathrm{ml} / \mathrm{min} / 1.73 \mathrm{~m} 3)$ was $9.4 \%$ demonstrating that the rate of cardiorenal syndrome in this population is low. In South African setting, HIV related $\mathrm{CMO}$ occurs predominantly in a younger population and 
are more likely to be female. As compared to other forms of $\mathrm{CMO}$, the left ventricular dimensions are smaller with a high prevalence of anaemia. Early commencement of anti-retroviral therapy, irrespective of CD4 count, is mandatory in these patients.

Key words: HIV, cardiomyopathy

\section{CS/TH-P-8: CARDIAC PRESENTATION OF HIV POSITIVE PATIENTS IN AN URBAN HOSPITAL IN SOUTH AFRICA}

D Zachariah, K McCutcheon, A Vachiat, N Tsabedze, R Ramjee, J Moosa, T Maluleke, G Muskesimana, Mathenjwa P, M Ewing, V Paton, P Manga

School of Clinical Medicine, Division of Cardiology, Charlotte Maxeke Johannesburg Academic Hospital, Faculty of Health Sciences, University of the Witwatersrand, Johannesburg, South Africa

The aim of the study was to ascertain the spectrum of cardiac disease in HIV positive patients presenting to a busy urban tertiary hospital in South Africa. A prospective, clinical registry captured data from all de novo cases of heart disease presenting to our cardiology unit. A total of 6100 cardiac patients were admitted to the unit from August 2011 to September 2015. All HIV positive patients' data were analysed to delineate the profile of cardiovascular disease in these patients. There were 597 patients (9.8\%) who were confirmed to be HIV positive with three quarters of these patients with a CD4 count $<350 \mathrm{cells} / \mathrm{Ul}$. The mean age was 41 years (SD \pm 11 ) with 324 females (54.3\%). The most common presentation was HIV related cardiomyopathy (34.2\%). Pericardial disease was present in $12.5 \%$ of patients and pulmonary hypertension was seen in $7 \%$ of the cohort. Valvular disease accounted for $15.4 \%$, hypertensive heart disease was seen in $7.5 \%$ and $10.7 \%$ of patients had coronary artery disease. The minority of cases included; infective endocarditis (3.2\%), peri-partum cardiomyopathy $(3.2 \%)$, conduction disease (1.6\%), aortic disease (1\%), and congenital heart disease (1.8\%). Only twenty nine percent of the cohort was on anti-retroviral therapy at presentation. In South Africa HIV positive patients with cardiac disease present at a young age and are more likely to be female. A significant number of these patients have low CD4 counts and only a quarter of patients are on antiretroviral disease.

Key words: HIV, cardiac, manifestation

\section{CS/TH-P-9: SODIUM HYPOCHLORITE ACCIDENT: THE REALITY OF TOXICITY}

Ebrahim Patel, Megna Gangadin, Saidah Tootla

School of Oral Health Sciences, Department of Oral Rehabilitation, University of the Witwatersrand

The cytotoxicity and dangers of sodium hypochlorite (NaOCl) during endodontic treatment have been well documented. Whilst clinicians may award $\mathrm{NaOCl}$ the caution it deserves, dental students are at times oblivious to the catastrophic events that occur following its extrusion into adjacent soft tissues. A case is presented whereby a patient was referred for endodontic treatment in a mandibular lateral incisor. Incorrect access cavity preparation led to an undiagnosed perforation through which $\mathrm{NaOCl}$ was injected directly in to the soft tissues. Pain and necrosis followed, with a sequelae of tooth loss and communication between the oral cavity and sublingual fascial space. The timeline of events from extrusion to healing will be presented.

Key words: Sodium hypochorite, accident, toxicity

\section{CS/TH-P-10: DEVELOPMENT AND VALIDATION OF AN INSTRUMENT FOR LABOUR EPIDURAL ANALGESIA RECORDKEEPING IN HOSPITALS IN SOUTHERN GAUTENG}

Elizabeth Jacobs, Helen Perrie, Estie Mostert, Juan Scribante, Sean Chetty

Department of Anaesthesiology, School of Clinical Medicine, Faculty of Health Sciences, University of the Witwatersrand, Johannesburg, South Africa

Adverse events are a potential outcome, no matter how skilled the anaesthetist, or how stringently he/she observes protocol. The medical notes made after regional procedures are poor in comparison with those made for general anaesthesia, and this has come under scrutiny in recent years due to increased patient awareness, medical insurance billing strategies and the presence of regulatory bodies. Currently, different labour epidural anaesthesia records are used in the hospitals affiliated to the Department of Anaesthesiology at the University of the Witwatersrand. There is a need for standardised labour epidural anaesthesia records that comply with the minimum standards of the Health Professions Council of South Africa. The aim of this study was to develop and validate an instrument for labour epidural analgesia recordkeeping, using Lynn's model. A concept instrument, identifying items considered important on anaesthetic records was developed through a literature review and refined by a peer group discussion with 10 local experts. Items were added, removed or changed. The instrument was then e-mailed to 12 national experts for rating. Content validity rating of the whole instrument was 0.8 . Variations in individual patients and their labour epidural analgesia requirements, as well the way in which health care practitioners respond to those variations is undisputable. However, there is a basic core of information which must be documented. This study has identified the information that South African experts in labour epidural analgesia feel will constitute that core.

Key words: Labour, epidural, analgesia, recordkeeping 


\section{CS/TH-P-11: SUCCESSFUL ENDO- VASCULAR TREATMENT OF A TRAUMATIC CAROTID CAVERNOUS FISTULA PRESENTING WITH TORRENTIAL EPISTAXIS}

Elton Ncube', Thando Ncube², Victor Mngomezulu³, Victor Mngomezulu ${ }^{4}$, Steve Moeng ${ }^{5}$

'School of Clinical Medicine, Department of Radiology, University of the Witwatersrand, ${ }^{2}$ Radiology Registrar, Charlotte Maxeke Johannesburg Academic Hospital , ${ }^{3}$ Trauma Medical Officer, Charlotte Maxeke Johannesburg Academic Hospital , ${ }^{4} \mathrm{Head}$ of Radiology Department, Charlotte Maxeke Johannesburg Academic Hospital, ${ }^{5}$ Head of Department Trauma Surgery, Charlotte Maxeke Johannesburg Academic Hospital

Traumatic carotid cavernous fistulae (CCF) are a rare pathology presenting in various ways including severe epistaxis. We present a polytrauma 38 year old male unrestrained driver involved in a high velocity collision with a concrete wall. He was admitted in the trauma intensive care unit with severe facial fractures and intracranial haemorrhages. He had marked periorbital swelling and chemosis on the left side. On the second week of admission, his stay was complicated by two episodes of severe torrential epistaxis managed emergently with nasal Foley's catheter tamponade, packing and blood transfusion. Computerised tomography angiograms revealed an established cerebral infarct and a left sided traumatic carotid cavernous fistula. Arteriography confirmed a left sided CCF, demonstrated some collateral flow. Embolisation of the carvenous sinus was not successful and we proceeded with endovascular coiling of the left internal carotid artery. Ocular symptoms resolved and there was no recurrence of epistaxis.

Conclusion: Our case highlights the potential complications that arise from severe skull base and craniofacial trauma. The clinician should have a high index of suspicion when faced with the typical physical signs, taking advantage of the latent period the CCF gives before manifesting its complications. Traumatic CCF should be recognised as a differential for severe epistaxis. Endovascular coiling can successfully be used to treat traumatic CCFs.

Key words: Endovascular, epistaxis, carotid cavernous fistula

\section{CS/TH-P-12: CHANGES IN HABITUAL PHYSICAL ACTIVITY AND SEDENTARY BEHAVIOUR IN PATIENTS WITH OSTEOARTHRITIS AFTER TOTAL KNEE ARTHROPLASTY - A PILOT STUDY}

Emmanuel Frimpong', David Goble', Joanne Alexandra McVeigh', Rebecca Mary Meiring'

'Exercise Laboratory, School of Physiology, Faculty of Health Sciences, University of the Witwatersrand, Johannesburg, South Africa, ${ }^{2}$ School of Occupational Therapy and Social Work, Curtin University, Perth, Western Australia.

Treatment for advanced knee OA requires a surgical approach. The most cost-effective surgical treatment for advanced knee OA is total knee arthroplasty (TKA). Currently there is no consistent and validated way of knowing whether functional ability improves after TKA. Moreover, there are no published data on objectively measured physical activity and sedentary behaviour of knee OA patients before and after TKA in South Africa. Therefore, the purpose of this study was to assess changes in patterns of objectively measured PA and SB before and after a unilateral primary total knee arthroplasty (TKA) in knee osteoarthritis (OA) patients. Eighteen knee OA patients, scheduled for primary TKA at the Charlotte Maxeke Johannesburg Academic Hospital (CMJAH), were recruited. PA and SB were measured two weeks prior to TKA (baseline) and six weeks post-TKA (post). PA and SB were assessed with an ActiGraph GT3X+ accelerometer. In addition, Body mass index (BMI) was assessed. Total volume and patterns of accumulation of PA and SB data were processed using a custom built SAS programme. Body mass index (BMI) decreased significantly post-TKA $(p=0.02)$. After adjusting for change in $B M l$, there were no significant changes in the total volume of time spent in PA and SB post-TKA ( $p>0.05)$. Breaks in prolonged sedentary time (> 20 min bouts) did not significantly change $(p=0.08)$ post-TKA. The number of sedentary bouts lasting between five and 10 minutes did not change significantly post-TKA compared to baseline $(p=0.094)$. Time spent in bouts of light activity lasting between 10 and 20 minutes did not change significantly post-TKA compared to baseline $(p=0.075)$.

Conclusion: This pilot study highlights the importance of assessing patterns of habitual physical activity and sedentary behavior after TKA as no changes in total volume of activity were observed, however, changes in the way the activity is accumulated may be different.

Key words: Physical Activity, Sedentary Behaviour, Total Knee Arthroplasty, Osteoarthritis

\section{CS/TH-P-13: NON-SEMINOMATOUS GERM CELL TUMOUR METASTATIC TO THE MAXILLA}

\section{Farai Zanamwe, Shabnum Meer}

Department of Oral Pathology, School of Oral Health Sciences, Faculty of Health Sciences, University of the Witwatersrand, Johannesburg, South Africa

This case report reviews germ cell tumours in the spectrum of metastatic disease to the oral cavity. Extragonodal germ cell tumours (GCT) primarily affect the sacrocccygeal region. GCT of the head and neck are rare accounting for only $3 \%$ of paediatric neoplasms. Only isolated cases of metastatic GCT have been reported. Benign teratomas are the most common extragonodal tumours of childhood, followed by yolk sac tumours which frequently arise in the ovaries and testis. Case study: a thirteen-year old male presented with a fairly rapidly enlarging, painless, 
pedunculated mass of the right maxillary tuberosity. The growth had been present for four months, and was haemorrhagic with focal necrotic areas. The tumour was noted extending from the buccal gingival mucosa to the hard and soft palate posteriorly. Ipsilateral submandibular lymphadenopathy was evident. No pathology was noted on the panelipse and periapical radiographs. The clinical impression was that of a lymphoma or Kaposi sarcoma. Histological examination of an incisional biopsy showed an extensively infiltrative high grade malignancy comprising predominantly of small, round, blue, discohesive tumour cells demonstrating a solid growth pattern, with areas of adenocarcinoma and squamous differentiation with keratinisation. Surface dysplasia was not present. Brisk mitotic activity was noted. Immunohistochemistry revealed positivity with for AEI/AE3, SALL4, Glyplican 3, and $\mathrm{OCT} 3 / 4$, confirming the diagnosis of a metastatic non-seminomatous GCT of mixed phenotype.

Key words: Extragonodal Germ, Cell Tumours, Teratoma, Yolk Sac Tumour

\section{CS/TH-P-14: THE ASSOCIATION BETWEEN PLACENTAL HUMAN PAPILLOMAVIRUS DETECTION AND PRE-ECLAMPSIA}

Francois Retief', Etienne Müller ${ }^{2}$

'Department of Obstetrics and Gynaecology, School of Clinical Medicine, Faculty of Health Sciences, University of the Witwatersrand, Johannesburg, South Africa; ${ }^{2 N a t i o n a l ~ I n s t i t u t e ~}$ for Communicable Diseases

Pre-eclampsia is a common, serious complication of pregnancy of complex aetiology that to date has not been fully described. Human papillomavirus (HPV) is a ubiquitous, double-stranded DNA-virus that displays tropism for human mucosal and cutaneous epithelial tissues. An association between placental HPV infection and preterm labour and pregnancy loss has been described. Evidence supporting an association between HPV infection and pre-eclampsia has been published. In this case-control study, women with clinically apparent pre-eclampsia were matched to healthy controls. All subjects were delivered by caesarean section. In all subjects cervical and placental samples were collected at the time of Caesarean section. These samples were tested for HPV using a polymerase chain reaction (PCR) assay. In addition, the serum levels of soluble FMS-like tyrosine kinase (s-Flt) and placental growth factor (PIGF) were tested at the time of delivery. While clinically apparent disease was associated with increased levels of s-Flt and decreased levels of PIGF, HPV was not detected in any of the placental specimens using the PCR assay. As a result, no association was found between placental HPV detection and clinically apparent pre-eclampsia or deranged serum levels of s-FIt or PIGF.

Key words: Pre-eclampsia, human papillomavirus

\section{CS/TH-P-15: THE MORPHOMETRIC DESCRIPTIONS OF THE THORACIC VERTEBRAL PEDICLES IN SOUTH AFRICAN POPULATIONS}

Hassan Sani', Pedzisai Mazengenya', Pilani Nkomozepi

'School of Anatomical Sciences, Faculty of Health Sciences, University of the Witwatersrand, ${ }^{2}$ Department of Human

Anatomy and Physiology, Faculty of Health Sciences, University of the Witwatersrand, Johannesburg, South Africa

The use of pedicle screws as fixation devices for posterior spinal fusion surgery has become increasingly popular worldwide. They offer rigid segmental fixation after decompression and arthrodesis for various disorders of the spine. Most of the studies on the morphometry of the thoracic vertebral pedicles have been reported in European population, with a few reports in Asian populations and none in African populations. Previous studies had shown significant population and ethnic differences in pedicle morphometry. In the current study we assessed the pedicle dimensions at the isthmus in 60 thoracic vertebrae each of European, African and Mixed populations in South Africa obtained from the Raymond A Dart Collection housed in the School of Anatomical Sciences. The measurement taken at the isthmus of the vertebral pedicle included the transverse diameter, sagittal diameter, transverse angle, sagittal angle and chord length. The pedicular width was found to decrease from $\mathrm{T} 1$ to $\mathrm{T} 6$ and then increased gradually to $\mathrm{T} 12$. The pedicular height increased from $\mathrm{Tl}$ to $\mathrm{T} 12$. The transverse angle increased sharply from $\mathrm{Tl}$ to $\mathrm{T} 6$ and then decreased gradually to $\mathrm{T} 12$ in European and African populations whereas in the mixed population the decrease was noticed from $\mathrm{Tl}$ to $\mathrm{T} 6$ and increased to T12. The sagittal angle was constant and the chord length increase from $\mathrm{T} 1$ to $\mathrm{T} 12$ in all the populations. On all the pedicular measurements there was no statistically significant differences observed in all populations studied. This information is vital in determining the safety margin of transpedicular fixation in our populations.

Key words: Thoracic pedicle, Morphometry, Pedicle Screw, South African Population

\section{CS/TH-P-16: SPONTANEOUS BILATERAL POSTERIOR HIP DISLOCATIONS IN AN ADULT MALE; A RARE PHENOMENON}

Ike Egbunike', Thando Ncube ${ }^{2}, K_{\text {Khodi Sikhauli }}^{2}$, Gerard Karera ${ }^{3}$, Mmampapatla Ramokgopa ${ }^{4}$

'School of Clinical Medicine, Department of Orthopaedic Surgery, ${ }^{2}$ Orthopaedic Registrar, Chris Hani Baragwanath Hospital, ${ }^{3}$ Consultant Orthopaedic Surgeon Chris Hani Baragwanath Hospital, ${ }^{4} \mathrm{Head}$ Orthopaedic Surgery Chris Hani Baragwanath Hospital

Hip dislocations in adults are often a sequel to high energy trauma in motor vehicle accidents and falls. Spontaneous hip dislocations have been reported in children with hip dysplasia, cerebral palsy and septic arthritis. We report a 
rare case of spontaneous bilateral hip dislocations in a 38 year old HIV positive male patient. We review the literature surrounding this unusual presentation.

Key words: Spontaneous, hip, dislocations, tuberculosis

\section{CS/TH-P-17: STROKE ADMISSIONS IN PATIENTS WITH ATRIAL FIBRILLATION TAKING NOVEL ORAL ANTICOAGULANTS (NOACS) COMPARED TO WARFARIN. A RETROSPECTIVE MEDICAL SCHEME DATABASE ANALYSIS}

Jacqui Miot', Susan Smith², Niri Bhimsan²

'Department of Pharmacy and Pharmacology, School of Therapeutic Sciences, Faculty of Health Sciences, University of the Witwatersrand, Johannesburg, South Africa; ${ }^{2}$ Clinical Policy Unit, Discovery Health

Patients with atrial fibrillation (AF) are at increased risk of ischaemic strokes. Warfarin has been shown to reduce the stroke risk in patients with $\mathrm{AF}$, however treatment with warfarin requires regular internal normalised ratio (INR) monitoring and is associated with food and medicine interactions. Newer anticoagulation agents (NOACs) have recently become available and have generally shown non inferiority to warfarin in stroke prevention with fewer adverse events such as bleeding and do not require regular monitoring. However, the NOACs are considerably more expensive than warfarin. Real world data on outcomes with the NOACs is lacking in South Africa and therefore the aim of this analysis was to compare the rate of hospital admissions for all strokes and gastric bleeding for patients in a large private medical scheme with nonvalvular AF taking either warfarin or NOACs. A retrospective database analysis was carried out on a cohort of patients who had up to two years exposure following treatment with either warfarin ( $n=1153)$ or a NOAC $(n=537)$ and who had a CHADS, score (Congestive heart failure, Hypertension, Age $\geq 75$ years, Diabetes mellitus, Stroke) of $\geq 2$. Patient demographics showed similar age, gender, history of previous stroke and $\mathrm{CHADS}_{2}$ score between the groups. A cox proportional hazard model showed no statistically significant difference in stroke admissions between the NOAC and warfarin patients (HR 0.6805, 95\% Cl 0.4154, 1.115). Patients who had a prior history of stroke were more likely to have another stroke in the analysis period.

Key words: Stroke, NOAC, Atrial Fibrillation

\section{CS/TH-P-18: HISTONE DEACETYLASE INHIBITOR: ROLE ON ONCOGENIC MICRORNA EXPRESSION ON COLON CANCER CELL LINES}

Jeyalakshmi Kandhavelu, Kumar Subramanian, Amber Khan, Paul Ruff, Clement Penny

Department of Internal Medicine, School of Clinical
Medicine, Oncology, Faculty of Health Sciences, University of Witwatersrand, Johannesburg, South Africa

Epithelial cancers, such as colorectal cancers are highly predominant in the Southern African population. MicroRNAs (miRNAs) are small, non-coding RNAs, which regulate approximately $30 \%$ of the transcriptome, affecting many cellular functions in both normal and neoplastic cells, by controlling the expression of their target genes. However, in human tumours miRNA target genes may be epigenetically silenced by altering chromatin status through histone modifications, or by aberrant hypermethylation of CPG islands that either incorporate or are adjacent to such miRNA genes. Moreover, histone deacetylases (HDACs) have been identified as key histone modifiers of deregulated miRNAs in cancer, through histone deacetylation and methylation, acting to stimulate or suppress miRNA expression. Histone deacetylase inhibitors (HDACi) are known to induce growth arrest, differentiation and apoptosis in several cell lines, including colon cancer. Suberanilohydroxamic acid (SAHA) is a Food and Drug Administration (FDA) approved HDACi, for the treatment of cutaneous T-cell lymphoma. Since the regulatory mechanism of histone modification on miRNA is poorly comprehended, using the HT29 and DLDI colon cancer cell lines, we assess the effects of SAHA on the expression levels of a number of candidate miRNAs identified in silico using miRNA target prediction tools. Next, the IC50 of the SAHA was calculated to range between 2.5 to $5 \mu \mathrm{M}$, using cell viability assays. Presently, changes in miRNA expression profiles are being assessed in the cells in response to $2.5 \mu \mathrm{M}$ and $5 \mu \mathrm{M}$ concentrations of SAHA. This study may clarify how histone modification in colon cancer and the anti-tumour effects of HDACi does regulate miRNAs.

Key words: Colon cancer, miRNA, SAHA, cell viability

\section{CS/TH-P-19: BETA-BLOCKER TARGET DOSING AND TOLERABILITY IN A DEDICATED HEART FAILURE CLINIC: CHARLOTTE MAXEKE JOHANNESBURG ACADEMIC HOSPITAL- 2000-2014.}

Jonathan Bolon, Keir McCułcheon, Pravin Manga, Eric Klug, Darryl Smith

Department of Internal Medicine, Division of Cardiology, Faculty of Health Sciences, University of the Witwatersrand, Johannesburg, South Africa

The benefit of Beta-blockers in chronic heart failure with left ventricular dysfunction is well established. However, actual use in "real world" heart failure patients has been relatively poor. The aim of this study was document betablocker tolerability in a dedicated heart failure clinic at Charlotte Maxeke Johannesburg Academic Hospital and to assess the proportion of patients who may require Ivabradine therapy. The records of all patients attending the heart failure clinic between 2000 and 2014 were reviewed. Demographic, clinical and outcome data 
was recorded for 500 patients. At their last clinic visit, 489 out of $500(97.80 \%)$ patients were taking a beta-blocker. Patients were stratified into categories according to guideline target doses, with $59.8 \% \quad(n=299)$ achieving 'target dose', $28.0 \% \quad(n=140)$ a 'moderate' dose, $5.4 \%$ $(n=50)$ receiving 'low dose' of beta-blocker and 11 patients $(2.2 \%)$ no dose. Sixty one $(7.6 \%)$ patients had Beta-blocker "intolerance". Conventional reasons for beta-blocker caution (bronchospasm/breathlessness, syncope, cardiac decompensation, hypotension) were found to be rare. Bradycardia was the commonest cause of inadequate uptitration. Ultimately only 53 patients $(10.6 \%)$ were deemed to be "ivabradine suitable".

Conclusion: Beta-blockers are well tolerated with perceptions around intolerability and concerns about safety largely unsupported by our experience. As a consequence, the role for Ivabradine therapy in patients with chronic heart failure is limited.

Key words: Beta-blockers, Heart Failure

\section{CS/TH-P-20: CERVICAL ABNORMALITIES AMONGST PARTICIPANTS SCREENED FOR THE MTN020/ASPIRE TRIAL IN HILLBROW, JOHANNESBURG}

Krishnaveni Reddy, Nerusha Govender, Helen Rees, Thesla Palanee-Phillips

School of Clinical Medicine, Wits Reproductive Health and HIV Institute (WRHI), Faculty of Health Sciences, University of the Witwatersrand, Johannesburg, South Africa

The ASPIRE study was a safety and effectiveness trial of a dapivirine containing vaginal ring for prevention of HIV-1 infection in women. We report data on cervical abnormality prevalence among participants screened at Wits RHI Hillbrow clinical research site. Between 19 October 2012 and 30 May 2014, 328 sexually active HIVuninfected, non-pregnant women [median age (MA) 28 years] had cervical samples collected for Papanicolaou (Pap) smear analyses during screening visits. Samples were assessed using liquid based cytology and graded using the Bethesda classification system. Cervical abnormalities were observed in $11.6 \% \quad(n=38)$ of 328 women with 2 (0.6\%), 19 (5.8\%), $11(3.4 \%)$ and 6 (1.8\%) women having evidence of high grade squamous intraepithelial lesion (HSIL), low grade squamous intraepithelial lesion (LSIL), atypical cells of undetermined significance (ASCUS) and atypical squamous cells cannot exclude HSIL (ASC-H) respectively. Of the $11.6 \%, 5.5 \%(n=18)$ were not enrolled into ASPIRE due to HSIL or ASC-H classification ( $n=8$, MA was 33.5 years) or other ineligibility related reasons $(n=10)$ and referred for public sector care. The remaining $6.1 \%$ women with LSIL and ASCUS ( $n=20$ ); MA (28 years) were enrolled and clinically managed during the study. In this population, HSIL prevalence was low and ASCUS and LSIL abnormalities were most common. These data are consistent with evidence that women $<30$ years tend to present with low frequency of high grade abnormalities but supports Pap smear testing in research trials to facilitate early abnormality detection, referral and management.

Key words: ASPIRE, Pap, Cervical Abnormalities

\section{CS/TH-P-21: EFFECT OF THE DEMETHYLATION AGENT, ZEBULARINE AND THE MICROTUBULE DISRUPTOR, PACLITAXEL ON MICRORNA EXPRESSION LEVELS IN BREAST CANCER CELL LINES}

Kumar Subramanian, Jeyalakshmi Kandhavelu, Amber Khan, Paul Ruff, Clement Penny

Department of Internal Medicine, School of Clinical Medicine, Oncology, Faculty of Health Sciences, University of the Witwatersrand, Johannesburg, South Africa

MicroRNAs (miRNA) are small, noncoding RNAs that are key regulators of gene expression during development; and are recurrently and differentially expressed in human disease states, in particular cancer. The present study evaluates the effects of the demethylation agent, zebularine and the microtubule disruptor, paclitaxel on potential oncogenic miRNA expression in the hormone insensitive triple negative breast cancer (TNBC) cell line, MDA-MB-231 relative to the hormone-sensitive MCF7 breast cancer cell line. The IC50 of Paclitaxel varied between 10-100nM, while for zebularine, it ranged from 200-400 $\mu \mathrm{M}$, after $48 \mathrm{hrs}$ of treatment. Also, the MDAMB-231 cell line was found to be pharmacologically more sensitive than MCF-7 cells. After treatment of these cell lines with each of these drugs, the expression of two significant oncogenic miRNAs, miRNA-21 and -155, were evaluated. When compared to untreated cells, quantitative expression analysis by QRT-PCR, revealed that zebularine downregulated miRNA-155 in MCF-7 and MDA MB-231 cells 1.15 and 1.03 fold, respectively. MiRNA-155 was expressed 2.4 fold higher in MDA MB-231, when compared to MCF-7 cells. The effect of paclitaxel on miRNA expression is yet to be confirmed. Thus, these findings implicate miRNA-21 and -155 as oncogenic miRNAs (oncomirs) which act to repress specific tumor suppressors. Both miRNA-21 and-155, are proposed here as novel molecular targets for TNBC cancer therapy.

Key words: Breast cancer, microRNA, Paclitaxel, RT-PCR, Zebularine

\section{CS/TH-P-22: THE ROLE OF FAECAL MICROBIOTA TRANSPLANTATION IN RESISTANT CLOSTRIDIUM DIFFICILE DIARRHOEA}

Seohee Lee, Katherine Drennan, Gizelle Simons, Amy Hepple, Kay Karlsson

School of Clinical Medicine, Wits Donald Gordon Medical

Centre, Faculty of Health Sciences, University of the Witwatersrand, Johannesburg, South Africa

Faecal microbiota transplantation (FMT) is a successful treatment for antibiotic-resistant Clostridium difficile 
(ARCD) infection. Donor stool is transplanted to repopulate the gut with beneficial flora. No studies to determine the efficacy of FMT in South Africa have been published. The aim was to identify demographics, risk factors and poor prognostic factors associated with ARCD infection and evaluate the success of FMT using different modes of administration at Wits Donald Gordon Medical Centre (WDGMC) between 2012 and 2015. A retrospective record review was conducted on patients who underwent FMT for ARCD at WDGMC. Data were collected regarding the demographics, clinical presentation and associated risk factors in patients as well as the route of administration of the FMT and outcome. From a sample of 19 patients, $13 / 19$ (68.4\%) were females and 6/19 (31.6\%) males. Elderly females predominated and the majority were over the age of 60 years $(9 / 13 ; 69.2 \%)$. Most common risk factor was underlying inflammatory bowel disease (8/19; 42.1\%). Most common method of FMT was via nasogastric tube (13/19; 68.4\%). Laboratory studies four weeks post-FMT showed that 15/19 (79.0\%) had a negative Clostridium difficile result. The remainder did not have a test $(4 / 19 ; 21.1 \%)$, verbal follow up confirmed clinical resolution of the diarrhoea. In conclusion, the demographics of patients referred for FMT at WDGMC reflected international data. Inflammatory bowel disease was the most common risk factor and also as a poor prognostic factor. An outcome of FMT at WDGMC was highly successful, regardless of the mode of administration.

Key words: Clostridium difficile, Faecal transplantation

\section{CS/TH-P-23: USING A SURROGATE VACCINE TO ASSESS FEASIBILITY AND ACCEPTABILITY OF INNER-CITY MEN IN THE PREPARATION FOR A FUTURE HIV VACCINE TRIAL IN JOHANNESBURG, SOUTH AFRICA}

Lucy Chimoyi, Mphatso Kamndaya, Emilie Venables, Nina von Knorring, Jonathan Stadler, Catherine MacPhail, Matthew Chersich, Helen Rees, Sinead Delany-Moretlwe

Wits Reproductive Health and HIV Institute (WRHI), Faculty of Health Sciences, University of the Witwatersrand, Johannesburg, South Africa

Developing an effective HIV vaccine is priority for HIV prevention. Enrolling and maintaining a cohort of men into HIV vaccine trials will promote development and licensure of safe and efficacious vaccines. This twelvemonth study enrolled and followed-up 150 consenting HIV and Hepatitis B negative, sexually active adult men into a pilot 1:1 randomised-controlled trial of immediate vaccination (IV) with hepatitis B vaccine compared to deferred vaccination (DV). Retention was descriptively assessed every month, change in risk behaviour and sideeffects post-vaccination quarterly and acceptability at study exit. Men were aged median 27(8), employed (53\%), secondary school educated (88\%) and uncircumcised (67\%). Overall follow-up time for 135 men was 1598.3 person-months with 15 withdrawals. High visit (89\%) and vaccine $(91 \%)$ completions were reported. Few mild post-vaccination adverse events were reported (10\%). Low levels of risk behaviour were observed throughout the study. Acceptability of trial procedures and services as well as willingness to participate (96\%) in future trials was high. Randomisation, informed consent and examination by female healthcare provider were least favourable. Quality health care services provided an important incentive for participation. Main motivations for participation were HIV prevention benefits $(81 \%)$ and altruism (75\%) with reimbursements ranking much lower (2\%). Safety concerns (98\%) were cited as a potential barrier to participation. Findings from this study show a high willingness to participate in future vaccine trials. The study shows that while access to vaccines is important, quality health services are equally an important incentive for enrolment.

Key words: HIV, Vaccine, Men, Inner-city

\section{CS/TH-P-24: THE USE OF A NOVEL POLYELECTROLYTE COMPLEX TO ENHANCE THE AQUEOUS SOLUBILITY OF A BCS CLASS II ANTI-HIV DRUG}

Margaret Siyawamwaya, Viness Pillay

Department of Pharmacy and Pharmacology, School of Therapeutic Sciences, Wits Advanced Drug Delivery Platform, Faculty of Health Sciences, University of the Witwatersrand, Johannesburg, South Africa

Poor in vivo bioavailability of drugs in Biopharmaceutics Classification System (BCS) class II remains a cause for concern in anti-HIV therapy. It is imperative to enhance the bioavailability of these drugs in order to circumvent the need for large doses which leads to toxicity in the body. A novel polyelectrolyte complex was utilised to improve the solubility and therefore, the bioavailability of a poorly water soluble anti-HIV drug, efavirenz, which has a bioavailability of $40-45 \%$. Solid dispersions of the drug in the polyelectrolyte complex were synthesised. Characterisation of the drug and polymeric complex were undertaken with the aid of Attenuated Total ReflectanceFourier Transform Infrared (ATR-FTIR), scanning electron microscopy (SEM) and differential scanning calorimetry (DSC). Human colorectal adenocarcinoma (Caco2) cell line viability test was conducted to determine the biocompatibility of the system. Any cytotoxicity caused by the efavirenz loaded complex over the 24 hour test period was measured and quantified. In order to determine any increase in solubility and therefore bioavailability of the drug entrapped in the complex, a saturation solubility study was carried out. The complexation ability of the 
two polymers was attributed to their complementary charges that resulted in the formation of the electrostatic bonds between the polymers. Efavirenz was successfully dispersed and entrapped in the polyelectrolyte complex. The study conclusively revealed that the solubility of efavirenz loaded into the optimised polyelectrolyte complex was superior by $14,16 \%$ to that of the comparator product tested in simulated human gastrointestinal fluid.

Key words: Polyelectrolyte Complex, Solubility, Bioavailability, Efavirenz

\section{CS/TH-P-25: LEARNING TO USE THE ONE-SIZED SILCS DIAPHRAGM IN SOUTH AFRICA: IMPROVEMENTS AFTER 5 USES}

Mags Beksinska', Jennifer A. Smit', Busi Maphumulo', Ross Greener', Nonhlanhla Mphili', Jennifer Foster' ${ }^{2}$, Maggie Kilbourne-Brook ${ }^{2}$

'Department of Obstetrics and Gynaecology, School of Clinical Medicine, MatCH Research Unit (MRU), Faculty of Health

Sciences, University of the Witwatersrand, Johannesburg, South Africa, ${ }^{2}$ Program for Appropriate Technology in Health (PATH)

The SILCS Diaphragm is a single-size, reusable contraceptive developed through a user-centered process. If used in conjunction with an effective microbicide gel, the SILCS and gel could protect from both unintended pregnancy and HIV/STIs. The objectives of this study were to assess the ability of women in a public-sector setting in South Africa to successfully fit and learn to use the SILCS diaphragm, and to evaluate changes in ease-of-use and reported problems over the first five uses. This was a randomised, crossover study among 115 women in Durban designed to assess the acceptability and preferences for the SILCS diaphragm used for gel delivery compared to gel delivery from prefilled applicator. Confidence about inserting/ using the SILCS, and perception about ease of use and acceptability were evaluated after one and five uses. A total of 115 women, aged 18 to 44 years, were enrolled and 106 (92\%) completed the study. Ease of insertion improved over time, with $72 \%$ reporting that the SILCS was easy to insert at first use, compared to $87 \%$ reporting ease of insertion at use five. Most (83\%) also felt comfortable after two to three insertions. Experience of gel leakage before sex reduced from $14.2 \%$ to $5.7 \%$ and leakage after sex declined from $2.8 \%$ at first use to $1.9 \%$ at fifth use. Womens' confidence of correct placement increased from $76.4 \%$ to $80.2 \%$ after five uses. These results show that with short-term training and counselling women can learn to fit and use the SILCS Diaphragm-even when women have never used a diaphragm before.

Key words: Diaphragm, reproductive health, pregnancy and HIV prevention
CS/TH-P-26: A RANDOMISED CROSSOVER TRIAL COMPARING THE MENSTRUAL CUP TO TAMPONS OR SANITARY PADS IN A LOW RESOURCE SETTING

Mags Beksinska', Jennifer A Smit', Ross Greener', Catherine S Todd $^{2}$, Mei Ling Ting Lee ${ }^{3}$, Virginia Maphumulo', Vivian Hoffman ${ }^{4}$

'School of Clinical Medicine, Department of Obstetrics and Gynaecology, MatCH Research Unit (MRU), Faculty of Health Sciences, University of the Witwatersrand, Johannesburg, South Africa; ${ }^{2}$ Family Health International FHI360 Asia Pacific Regional Office, Bangkok, Thailand, 3University of Maryland, School of Public Health, College Park, ${ }^{4}$ International Food Policy Research Institute

In low-income settings, women and girls face activity restrictions during menses due to lack of affordable menstrual products. The menstrual cup (MC) is a nonabsorbent reusable cup to collect menstrual blood. This method is cost effective compared to other sanitary products and impacts less on the environment, yet it is not widely used. To assess the ability of women in a publicsector setting in South Africa to successfully fit and learn to use the menstrual cup and to evaluate changes in easeof-use and reported problems over three menstrual cycles of use. This was a randomised, crossover study among 110 women in Durban, South Africa, Participants aged 1845 years with regular menstrual cycles, had water from the municipal system as their primary water source and had no sexually transmitted infections were eligible for inclusion. Participants used the menstrual cup over three menstrual cycles and were interviewed at baseline and monthly follow-up visits. Of 124 women assessed, 110 were eligible and randomly assigned to selected menstrual products. 105 women completed all follow-up visits. By comparison to pads/tampons (usual product used), the MC was rated significantly better for comfort, quality, menstrual blood volume collection, appearance, and preference. Both these comparative outcome measures and likelihood of continued use, recommending the product, and future purchase increased for the MC over time. In a population of novice users, initial concerns and user problems were overcome by almost all women over three-cycles of use.

Key words: Menstrual cycle, adolescent health, reproductive health

\section{CS/TH-P-27: THE CLINICAL PROFILE OF IDIOPATHIC PARKINSON'S DISEASE IN A SOUTH AFRICAN HOSPITAL COMPLEX- THE INFLUENCE OF RACE AND GENDER}

Marcelle Smith, Girish Modi

Department of Neurosciences, School of Clinical Medicine, Faculty of Health Sciences, University of the Witwatersrand, Johannesburg, South Africa

Idiopathic Parkinson's disease (IPD) has not been well studied in Black African populations. There are several studies attempting to address these issues. The aim of the 
study was to determine whether race and gender have any impact on the phenotype of IPD in our sample of patients. Participants known with IPD were recruited from the Neurology clinics at Chris Hani Baragwanath Hospital and Charlotte Maxeke Johannesburg Academic Hospital. The data collection was in the form of a questionnaire and clinical evaluation which included a mental status examination (MMSE), and illness staging. Fifty patients, previously diagnosed with IPD, were recruited. Thirtyfive patients were Black African, eleven were of White European descendant, three were of Indian descent and one had mixed ancestry. Twenty-eight of the patients were female. There were no significant gender differences within or between the different ethnic groups. Seventyone percent of Black and ninety-one percent of White participants had classic IPD presentations. A resting tremor was found in fifty-nine percent of all males in the study but in ninety-four percent of females. In the Black patients with IPD, thirty one percent had early onset IPD lage of onset less than 50 years) with a gender ratio of $M: F=1: 6$, compared to $18 \%$ of White patients. Twenty-nine percent of Black patients had an akinetic-rigid syndrome with erect posture and no tremor (gender ratio of $M: F=7: 4$ ) compared to nine percent of White patients. Cognitive impairment was more prevalent in Black patients $(74 \%)$ versus White patients (18\%). A weakness of the study was that we failed to recruit equal numbers of each ethnic group, due to the patient profile of the hospitals in which the study was conducted. In conclusion, the phenotype of IPD in the majority of our study population is of the classic IPD type. In a third of our Black patients the onset was early and in a third the presentation was akinetic. Cognitive impairment is also more severe in these patients.

Key words: Parkinson's, South Africa, Akinetic-rigid, Syndrome

\section{CS/TH-P-28: AN EVALUATION OF THE USE OF GRANULOCYTE COLONY STIMULATING FACTOR AS AN ADJUNCT TO IN VITRO FERTILISATION IN PATIENTS WHO HAVE PREVIOUSLY FAILED ATTEMPTS AT PREGNANCY WITH IN VITRO FERTILISATION}

Tasneem Mohamed, Yasmin Adam, Mohamed Iqbal Cassim

Department of Obstetrics and Gynaecology, School of Clinical Medicine, Faculty of Health Sciences, University of the Witwatersrand, Johannesburg, South Africa

For some, pregnancy by standard in vitro fertilisation (IVF) is not achieved, even if ovulation induction and embryo development is successful. This may be due to defective implantation. A thin endometrium is associated with implantation failure. Studies show that improved endometrial thickness increases the probability of successful IVF. The aim of the study was to evaluate the effects of granulocyte colony stimulating factor (G-CSF) as an adjunct to standard IVF. The study looked at the influence of G-CSF on achievement of pregnancy as well as its effects on the endometrium. This was a retrospective cross-sectional study of a subgroup of women attending BioART Fertility Centre, who had two or more failed IVFs previously. These women underwent a procedure of transvaginal instillation of G-CSF in addition to their IVF protocol. Endometrial thickness was not a criterion for its use. The group consisted of 49 women, mean age 38.9 (SD \pm 6.11 ). Mean number of previous IVFs were 3.1 (SD \pm 1.76 ). Mean endometrial thickness pre-GCSF was $7.53 \mathrm{~mm}$ (SD \pm 2.69) and post-GCSF was $9.11 \mathrm{~mm}(S D \pm 2.12)$. The clinical pregnancy rate was $34.69 \%$. Univariate analysis between those that achieved pregnancy and those that didn't showed that the age difference between the groups was statistically significant (p-value 0.0005). G-CSF use was associated with increased pregnancy rates in younger patients. Mean endometrial thickness pre and postGCSF between the groups was not statistically significant ( $p>0.05)$. However the mean change in endometrial thickness in all patients regardless of pregnancy outcome was statistically significant $(p=0.0029)$. G-CSF is a useful adjunct in the treatment of patients aged less than 38 years with recurrent failed IVFs. We reported a statistically significant overall expansion of endometrial thickness with the use of G-CSF but failed to show any association between endometrial expansion and pregnancy outcome.

Key words: G-CSF, IVF, endometrium, pregnancy

\section{CS/TH-P-29: HIV SELF-TESTING USABILITY ASSESSMENT IN AN ADULT HIV CLINIC IN JOHANNESBURG, SOUTH AFRICA}

\section{Mohammed Majam, Francois Venter}

School of Clinical Medicine, Wits Reproductive Health and HIV Institute, Faculty of Health Sciences, University of the Witwatersrand, Johannesburg, South Africa

Self-testing for HIV is a novel approach that has the potential to be a disruptive innovation that can aide in the reduction of the HIV testing gap. Several candidate devices are being readied for performance evaluation and acceptability in the South African market. Prior to field testing, the devices must undergo optimisation, both in terms of product design and with the instructions for use (IFU). This study investigated the usability of one such blood-based, finger-prick device in an Adult HIV Clinic in a SA Academic Hospital. A total of 100 participants were recruited across three education levels. Since this is a usability assessment and not a device performance evaluation, a successful self-test was defined as having completed all of the required steps correctly, irrespective of a final result. The observers noted any errors occurring within those critical steps, however, allowed participants 
to carry on with the process to completion observing each step in the process in isolation. A total of $60 \%$ of the participants managed to complete all critical steps correctly. In isolation, each critical step had between an 82-95\% success rate. The highest numbers of errors were made during the filling of the blood collection tube with $18 \%$ error. The IFU for Self-Testing are a critical component and ensuring ease of use is key to the success of the product. This usability assessment showed that an overall average of $88 \%$ of all steps was performed correctly. Device manufacturers now have the opportunity to analyse the data and remedy any ambiguity in the instructions.

Key words: HIV Self-Testing, Rapid Diagnostics

\section{CS/TH-P-30: ELIMINATION OF MOTHER- TO-CHILD TRANSMISSION OF HIV: GAPS IDENTIFIED IN URBAN PRIMARY HEALTH CARE FACILITIES IN JOHANNESBURG, SOUTH AFRICA}

Chandiwana N, Sawry S, Sanele Mdanda, Mvundla N, Saiqa Mullick, Lee Fairlie

Wits Reproductive Health and HIV Institute (Wits RHI), Faculty of Health Sciences, University of the Witwatersrand, Johannesburg, South Africa

Prevention of mother-to-child transmission (PMTCT) of $\mathrm{HIV}$ is the most successful HIV prevention intervention globally and in South Africa. However elimination has not been achieved. Given the ambitious targets to eliminate paediatric HIV worldwide, on-going assessment of PMTCT programmes in the postpartum period is critical. This study evaluated the uptake and potential gaps in PMTCT services in Johannesburg, South Africa. We conducted a cross-sectional study between 1st October 2015 and 31 st March 2016 at three primary care clinics in inner-city Johannesburg, South Africa. Post-natal women (>18years old) attending an immunisation visit with their infant $(<18$ months old) were eligible for inclusion. Enrolled women were interviewed on their uptake of comprehensive PMTCT services and all consenting mothers and infants of seropositive mothers were tested for HIV at study enrolment. Among 533 mother-infant pairs were enrolled; 281 (52\%) were HIV-positive at their last pregnancy. Mother-infant pairs enrolled at median infant age of 3.1 months. HIVpositive women were significantly older and were more likely to be unemployed than HIV-negative women. Only $69 \%$ of women were retested for HIV post-partum, with $5.1 \%$ subsequently testing positive at study enrolment. HIV-infected women were more likely to use condoms during pregnancy, $43.8 \%$ vs. $17.5 \%(p=<0.001)$ Maternal self-reported ART adherence was low (51\%). Only $46 \%$ of women had a viral load in the past 12 months.

Conclusion: We found many successes and a few missed opportunities in the PMTCT cascade in an urban high HIV burden setting. PMTCT coverage rates were high and vertical transmission low; however, most women were enrolled early in the postpartum period. Women and breastfeeding infants remain at high risk of HIV acquisition postnatally. Maternal ART adherence is low and viral load measurement inadequate. Increased efforts to reduce risky sexual behaviour, reinforce consistent condom use and promote safe infant feeding are vital postpartum Longitudinal studies that measure the incidence of HIV among mother-infant pairs and improves adherence to ART among HIV positive women are needed.

Key words: PMTCT, HIV, postpartum; mother-infant pairs

\section{CS/TH-P-31: A COMPARISON OF THE DIFFUSION KINETICS OF TOPICAL ANTI- GLAUCOMA DRUGS TIMOLOL AND PILOCARPINE}

Nomshado Zungu, Armorel van Eyk, Niel Butkow

Department of Pharmacology, Faculty of Health Sciences, University of the Witwatersrand, Johannesburg, South Africa

Glaucoma is characterised by an increase in intraocular pressure that leads to partial blindness and eventually complete vision loss if undetected. It is the second most common cause of vision loss globally following cataract formation. Timolol a $\beta$-adrenoceptor blocker and pilocarpine a parasympathomimetic and a muscarinic receptor agonist are commonly used as baseline treatment separately or in combination. The purpose of this study was to determine the in vitro diffusion characteristics across excised porcine and rabbit cornea of timolol and pilocarpine used for the treatment of glaucoma. The in vitro diffusion characteristics of the two compounds across excised porcine and rabbit corneas were determined using a PermeGear flow-through diffusion system with seven in-line flow-through cells. Each sample $(2 \%$ (w/v)) was loaded into the donor compartments and PBS $(\mathrm{pH}$ 7.4) pumped through the receptor compartments at $1.5 \mathrm{ml} / \mathrm{h}$. Samples were collected every $30 \mathrm{~min}$ over a 6-hour time period. Analyses performed utilising a RP-HPLC C18 column ( $220 \times 4.6 \mathrm{~mm}, 5 \mu \mathrm{m})$ with phosphate buffer (pH 3.5): methanol (70:30) for pilocarpine and (60:40) for timolol used as a mobile phase. Running conditions consisted of $10 \mu \mathrm{l}$ sample injected, 5 min running time at $1 \mathrm{ml} / \mathrm{min}$, room temperature and detection at $220 \mathrm{~nm}$. Method validation was performed according to $\mathrm{ICH}$ guidelines. The methods have shown good correlation coefficient $\left(\mathrm{R}^{2}\right)$, accuracy, precision, linearity, selectivity, specificity and intercept value. $R^{2}$ was 0,9932 and 0,9956 for pilocarpine and timolol respectively. Rabbit cornea seems to be more permeable to pilocarpine than porcine cornea in comparison to timolol.

Key words: Diffusion, HPLC Validation, Permeability 


\section{CS/TH-P-32: CAROTID ATHEROSCLEROTIC PLAQUE IN HUMAN IMMUNODEFICIENCY VIRUS POSITIVE PATIENTS WITH CRITICAL LIMB ISCHEMIA}

Nomvuyo Manyatsi', Taalib Monareng ${ }^{3}$, Angela Woodiwiss², Tshegofatso Motau', Chanel Robinson2, Imraan Ballim², Gavin Norton', Talib Abdool-Carim ${ }^{3}$

'School of Physiology, Faculty of Health Sciences, University of the Witwatersrand, Johannesburg, South Africa, ${ }^{2}$ Cardiovascular Pathophysiology and Genomics Research Unit, ${ }^{3}$ Faculty of Health Sciences, University of the Witwatersrand, Johannesburg, South Africa

In developed countries, Human Immunodeficiency Virus (HIV) is a recognised cause of occlusive arterial disease and this is atherosclerotic in nature. However, the role of atherosclerotic plaque in HIV-associated occlusive arterial disease in South Africa is uncertain. We aimed to compare the prevalence of atheroma (plaque and/or an increased carotid intima-media thickness [IMT]) in HIVpositive and negative patients with critical limb ischaemia (CLI). Fifty-four HIV-positive patients with CLI and $289 \mathrm{HIV}$ negative patients with CLI were studied. Carotid IMT and the presence of atherosclerotic plaque (identified as a focal structure that encroaches into the arterial lumen of at least $0.5 \mathrm{~mm}$ greater than the IMT, or demonstrates a thickness of $>1.5 \mathrm{~mm}$ as measured from the mediaadventitia interface to the intima-lumen interface) were determined using high resolution B-mode ultrasound technology. As compared to HIV-negative patients with CLI (63.4 \pm 10.9 years), HIV-positive patients were younger $(49.2 \pm 11.3, p<0.0001)$. The prevalence of plaque was $71.6 \%$ in HIV-negative patients whereas in HIV-positive patients it was only $25 \%, p<0.0005$. There were fewer HIVpositive patients (10.9\%) who had a carotid IMT $\geq 0.9 \mathrm{~mm}$ as compared to HIV-negative patients $(25.7 \%, p<0.02)$. When compared to 722 randomly selected participants (no CLI) from the community where only $4.2 \%$ participants had an IMT $\geq 0.9,23.3 \%$, ( $p<0.0001)$ of CLI had increased IMT. In conclusion, HIV-positive patients with CLI have a low prevalence of atheroma. Hence atherosclerosis may not be a major mechanism responsible for CLI in HIVpositive patients in South Africa.

Key words: Atherosclerosis, Human Immunodeficiency Virus, Critical Limb Ischaemia, Plaque

\section{CS/TH-P-33: THE PREVALENCE OF INCIDENTAL PHYSIOLOGICAL INTRACRANIAL CALCIFICATIONS IN THE ADULT SOUTH AFRICAN POPULATION AS SEEN ON COMPUTED TOMOGRAPHY}

Koranteng PN, Hlabangana LT

Department of Diagnostic Radiology, School of Clinical

Medicine, Faculty of Health Sciences, University of the Witwatersrand, Johannesburg, South Africa

Computed tomography (CT) is an imaging modality extensively used in radiology departments. Intracranial calcifications are incidentally seen on brain CT scans that are done for numerous reasons. These calcifications can be physiological or due to a pathological process. It is therefore essential for the reporting radiologist to be aware of areas of physiological calcifications to avoid incorrect diagnoses. South Africa has minimal data on the prevalence of physiological calcification compared to the rest of the world. The aim of this study was to determine the prevalence of physiological intracranial calcifications in the South African adult population as seen on noncontrasted brain CT. This was a retrospective study that evaluated 450 non-contrasted CT brain scans of adult male and female patients that met ascertained criteria for the presence of physiological calcifications. Pathological calcifications were also assessed. Overall, $98.2 \%$ of patients had some form of physiological calcifications with no significant association with gender. Choroid plexus calcifications were the most common (87.8\%), followed by pineal gland (76.0\%). Habenular and dural calcifications were found in $48.9 \%$ and $44 \%$ respectively. Petroclinoid calcifications were identified in $15.6 \%$, basal ganglia in $9.8 \%$ and arachnoid region in $2.9 \%$ of the study population. The least common calcifications were in the lens $(0.7 \%)$. Pathological calcifications were found in $8.7 \%$ of patients. In conclusion, the vast majority of patients had some form of physiological calcifications with no significant gender predilection. Choroid plexus were the most common, which was different from most other studies. Most of the compared studies concluded pineal calcifications to be the most prevalent. Pathological calcifications were uncommon.

Key words: physiological, intracranial calcifications, computed tomography

\section{CS/TH-P-34: EVALUATION OF HIV TESTING AND ANTIRETROVIRAL THERAPY INITIATION AT BIRTH: PRELIMINARY RESULTS FROM A PRIMARY AND SECONDARY CARE SETTING IN JOHANNESBURG, SOUTH AFRICA}

Nontuthuko Mvundla ${ }^{1,2}$, Nomathemba Chandiwana ${ }^{2}$, Lee Fairlie ${ }^{2}$, Ntombizethu Dumakude ${ }^{2}$, Nombuso Madonsela ${ }^{3}$, Esther Mabanga $^{3}$, Thembekile Ncube ${ }^{3}$

'School of Clinical Medicine, Faculty of Health Sciences, University of the Witwatersrand, Johannesburg, South Africa: ${ }^{2}$ Wits Reproductive Health and HIV Institute (WRHI), University of the Witwatersrand ${ }^{3}$ National Department of Health, South Africa

We describe the preliminary impact of very early infant diagnosis on combined antiretroviral therapy (CART) initiation and the feasibility of implementation in a primary (PHC) and secondary care setting. A retrospective review of all birth HIV PCRs was conducted at the labour wards of two large health care facilities in Johannesburg from 1st June to 31st December 2015. Infant birth HIV status was determined by heel prick HIV dried blood samples at the referring National Health Laboratory Service (NHLS) with results available within 48 hours. At the 3 -day postnatal 
visit, infants found to be HIV-positive were initiated on ART either at the facility's HIV clinic or referred to the nearest initiating facility. Of 2170 infants delivered at the PHC facility, $27 \%$ (576/2170) were HIV-exposed and tested at birth. Of these infants, $7(1.2 \%)$ had a positive HIV-DNA PCR. Of these, six infants were initiated on CART, with a median time to initiation of seven days. One infant was lost to follow-up despite vigorous tracing. Of 1701 infants delivered at the secondary facility, 18\% (318/1701) were tested for HIV at birth and $2(0.6 \%)$ were found to be HIVpositive. Preliminary results suggest that HIV birth testing at $\mathrm{PHC}$ level is both feasible and effective at very early infant diagnosis and treatment However, gaps in the referral systems at secondary level hindered early initiation of HIVpositive infants, ultimately undermining the goal of the new consolidated guidelines of birth testing.

Key words: Birth, HIV, testing, paediatrics

\section{CS/TH-P-35: DETERMINATION OF THE ADEQUACY OF CRANIAL ULTRASOUND REQUESTS AND REPORTS AT CHARLOTTE MAXEKE JOHANNESBURG ACADEMIC HOSPITAL AND RAHIMA MOOSA MOTHER AND CHILD HOSPITAL}

\section{Ntebogang Palesa Mutshutshu, Linda Tebogo Hlabangana}

School of Clinical Medicine, Department of Radiology, University of the Witwatersrand, Medical School

Cranial ultrasound is a cheap, effective and easy to use modality for the evaluation of cranial pathology in very sick paediatric population. It can be performed as a portable imaging investigation and repeated as many times as possible. New improvements in sonography equipment and technique make it possible for cranial ultrasound to compete with Cat scanners in terms of identifying pathology. The aim of this study was to determine the adequacy of cranial ultrasound requests and reports at Charlotte Maxeke Johannesburg Academic (CMJAH) and Rahima Moosa Mother and Child Hospitals (RMMCH) with regards to their completeness, accuracy and clinical relevance. A retrospective review of 191 cranial ultrasound requests and reports was performed at two academic centres. A data collection sheet was developed by the principal investigator and supervisor guided by literature with regards to the information required within the cranial ultrasound report. A scoring method was then developed with a maximum score of three given for the request adequacy and of 14 for report adequacy. Only $49.74 \%$ of the requests met the criteria for an adequate request. The mean report adequacy score was 7.03 with a standard deviation (2.02). Overall, $50.26 \%$ of the requests scored average and below average score. Results demonstrate that requests and reports of cranial ultrasound are not adequate at both centres. A cranial ultrasound template was therefore developed to assist with the standardisation of reports.

Key words: Cranial Ultrasound Requests, Reports

\section{CS/TH-P-36: EXPLORING DEMAND FOR THE V CONDOM IN SOUTH AFRICA}

Nzwakie Mosery', Mags Beksinska', Jennifer A. Smit', Cecilia Milford', Marion Stevens ${ }^{2}$, Maggie Kilbourne-Brook ${ }^{3}$, Patricia Coffey $^{3}$

'Department of Obstetrics and Gynaecology, School of Clinical Medicine, MatCH Research Unit (MRU), Faculty of Health Sciences, University of the Witwatersrand, Johannesburg, South Africa, ${ }^{2}$ Health Systems Trust, ${ }^{3}$ Program for Appropriate Technology in Health (PATH)

The $\mathrm{V}$ Condom is a female condom developed through a user-centered process, to be easy to use and provide good sensation for women and men. Market tests are being used to assess feasibility of commercial and publicsector distribution channels according to the total market approach, to determine how to position the product, and explore demand for it in South Africa. We assessed uptake and acceptability across multiple channels including university/college, health service centers, factory, and an HIV-prevention non-governmental organisation. Both qualitative and quantitative methods were used e.g. a promotional activity, distribution of condoms, baseline survey, completion of coital logs, SMS text message, focus group discussions and in-depth interviews. Here we report on the baseline data. Quantitative data were analysed in STATA and qualitative data analysed using QRS Nvivo. A total of 195 participants across three market tests completed baseline interviews. Most women (96\%) and men (95\%) used male condoms during the past three months, but fewer reported use at last sex $178 \%$ for women and $75 \%$ for men). The $V$ Condom was used in $61 \%$ of sex acts reported. The three most important reasons women gave for choosing the $\vee$ Condom were: liked packaging (is "stylish and charming" and "attracts your eye"); liked design and insertion is easy. Half the male sample (50\%), "wanted to try one." Most participants $178 \%$ of females and $80 \%$ of males) would be willing to pay for the $\checkmark$ Condom at prices ranging from 4.2 ZAR to 16.6 ZAR. Most participants reported preference for the $\mathrm{V}$ Condom over male and other female condoms. There is a commercialsector market for the $\mathrm{V}$ Condoms, and willingness to pay a price that approaches the estimated market price.

Key words: Female condom, public sector, commercial

\section{CS/TH-P-37: HIV AND TB IN PATIENTS ADMITTED WITH CONFIRMED VENOUS THROMBOEMBOLIC DISEASE - THE WELLS SCORE}

Pramodhini Moodley

Department of Internal Medicine, School of Clinical Medicine, Perinatal Research Unit, Faculty of Health Sciences, University of the Witwatersrand, Johannesburg, South Africa

HIV and TB confer increased risk for venous thromboembolic disease (VTE). Wells criterion is used to assess risk for deep vein thrombosis (DVT) and pulmonary embolism (PE) but does not include infection criteria. This study aims to report 
the proportions of in-patients with confirmed VTE (DVT and /or PE) with HIV infection and/or TB; and to report the Wells criteria and score by HIV serostatus. Patients admitted to Tshepong Hospital with DVT and/or PE were approached. The duration of symptoms, risk factors, HIV history and treatment, and TB history and treatment were recorded. We report the data of 66 patients; 55 had DVT (83.3\%), 7 PE (10.6\%) and 4 (6.1\%) both DVT and PE. Mean age was 48 years (standard deviation 14.75) with 41 (62\%) women; 39 (59.1\%) were HIV-infected; 22 had TB of whom virtually all (20) were co-infected with HIV. Twenty-four (36.4\%) had neither TB nor HIV. In the HIV-infected adults, 29 (43.9\%) were on ART, and nine were virally suppressed. Mean PE Wells score for HIV-seronegative and HIV-infected patients was 3.43 vs 5.38 respectively $(p=0.14)$. Mean DVT Wells score was 2.48 and 2.44 , respectively $(p=0.53)$. The three leading Wells criteria were similar by HIV serostatus for both DVT and PE. Both HIV infection and TB appear to be considerable risk factors for VTE which occurred across a range of CD4 counts and viral loads. Our data suggest that a TB and/or HIV criterion could be added to the Wells score.

Key words: HIV, TB, Venous Thromboembolism

\section{CS/TH-P-38: INFLUENCE OF FORMULATION AND PROCESS VARIABLES ON IN VITRO RELEASE OF METHOCARBAMOL FROM SPRAY-DRIED NANOPARTICLES}

\author{
Pride Mothobi, Viness Pillay
}

Department of Pharmacy, Wits Advanced Drug Delivery Platform, School of Therapeutic Sciences, Faculty of Health Sciences, University of the Witwatersrand, Johannesburg, South Africa

Establishing an in vitro-in vivo correlation is still one of the biggest challenges in research, despite many advances made. Other challenges include the selection of biocompatible and biodegradable polymers with desired properties to achieve therapeutic concentrations at the target site and designing drug delivery systems considering their predisposition to biodegradation. Methocarbamol nanoparticles were prepared from blended solutions of drug and $\mathrm{pH}$-dependent polymer combinations (Eudragit ${ }^{\circledR}$ L100-55 and Polyethylamine (PEI)) using a nano-spray dryer under predetermined operating parameters. A constant 1:1 (w/w) polymer/ polymer ratio was maintained. The effect of mixing order of the two polymers to the drug solution and drug-polymer blending time were evaluated. Each formulation of nanoparticles was dissolved in $\mathrm{pH} 7.4$ phosphate buffered saline (PBS) and diffusion studies were carried out using a vertical Franz cell arrangement and Strat- $M^{\mathrm{T} M}$ transdermal synthetic permeation membrane. Matrices blended for 5 minutes in PEl showed high drug entrapment and a sustained drug release profile, and enabled more than $10 \%$ drug release within two hours. In terms of the effect of polymer-drug mixing order, adding Eudragit@ L100-55 to the drug solution first showed higher drug release and permeation as opposed to the reverse.

Key words: Nanoparticles, in-vitro, drug

\section{CS/TH-P-39: AN AUDIT OF PATIENTS WITH COMPLEX REGIONAL PAIN SYNDROME AT THE HELEN JOSEPH HOSPITAL PAIN MANAGEMENT UNIT}

Rachelson Anthea ${ }^{1,2}$, Juan Scribante ${ }^{3}$, Helen Perrie ${ }^{3}, E_{\text {Eva Frohlich }}^{4}$

'Department of Anaesthesiology, School of Clinical Medicine,

Faculty of Health Sciences, University of the Witwatersrand,

${ }^{2}$ Mmed-Pain Management, ${ }^{3}$ Faculty of Health Sciences,

University of the Witwatersrand, Johannesburg, South Africa,

${ }^{4}$ Helen Joseph Hospital

Complex Regional Pain Syndrome (CRPS) consists of chronic pain and hyperalgesia, motor, autonomic and dystrophic disturbances. The aetiology and diagnosis are not clearly defined and to date few epidemiological studies have been carried out. The occurrence of CRPS at the Helen Joseph Hospital Pain Management Unit (HJHPMU) is not known and we undertook a study to describe the occurrence, profile and management of patients treated for CRPS at the HJHPMU. A retrospective audit of files of patients with CRPS was done for the time period 2005 to July 2014. Nine hundred and thirteen patients attended the unit during the study period and 42 (4.6\%) had a diagnosis of CRPS, 39 files were included in the study. Two patients' files didn't have consent and one was illegible. The demographic profile included age, gender, employment status, ethnicity and marital status. Orthopaedic surgeons referred the majority of patients (30.8\%). The most common inciting events were fractures and surgery. Management included pharmacotherapy comprising between two and five medications, at least one analgesic of which gabapentinoids were the most common (29.2\%). Physiotherapy was the most common $(35.1 \%)$ supportive therapy and a stellate ganglion block (38.1\%) was the most common interventional therapy. There is a small percentage of CRPS patients presenting for multidisciplinary pain management for CRPS in South Africa.

Key words: CRPS, pain management

\section{CS/TH-P-40: THE NATIONAL FEMALE CONDOM EVALUATION IN SOUTH AFRICA - 2013 CONDOM DISTRIBUTION STATISTICS}

Ross Greener', Mags Beksinska', Jennifer A. Smit', Zonke Mabude', Phumla Nkosi', Joanne Mantell', Muriel Kubeka

'Department of Obstetrics and Gynaecology, School of Clinical Medicine, MatCH Research Unit (MRU), Faculty of Health Sciences, University of the Witwatersrand, Johannesburg, South Africa; ${ }^{2}$ Department of Psychiatry, Columbia University

The first comprehensive evaluation of the SA programme is currently underway and aims to describe and evaluate 
approaches used in the programme to identify correlates/ predictors associated with uptake and continued use of female condoms. A situational analysis, conducted at the start of the evaluation, reviewed data from the 2013 District Health Information System for female condom (FC) and male condom (MC) distribution. Total FC and MC distribution per health facility and province were compared. Over 12 million FCs were distributed in 2013 which was $2.6 \%$ of the total proportion of MCs distributed over the same period. Over $90 \%$ of facilities in five provinces reported FC distribution in 2013, however not all facilities report data and data from a facility survey in the same study indicates higher levels of distribution. FC distribution percentages per province: Eastern Cape (76.7), Free State (87), Gauteng (92.7), KZN (92.9), Mpumalanga (90.8), Northern Cape (70.9), Western Cape (87.3), Limpopo (92.1), and North West (92.7). In conclusion, FCs are available throughout the public sector and the proportion of FC distribution compared to MCs is high in South Africa when compared to global distribution proportions of FCs and MCs (FCs currently account for $0.19 \%$ of global condom procurement).

Key words: Female Condom, DHIS, Distribution, Male Condom

\section{CS/TH-P-41: ATRIOVENTRICULAR FUNCTION ASSESSMENT IN CHRONIC RHEUMATIC MITRAL REGURGITATION: LOOKING BEYOND THE LEFT VENTRICLE}

Ruchika Meel 1,2, Elena Libhaber 2, Ferande Peters 1,2, Mohammed Essop ${ }^{1,2}$

'Division of Cardiology, Chris Hani Baragwanath Academic Hospital; ${ }^{2}$ Faculty of Health Sciences, University of the Witwatersrand, Johannesburg, South Africa

Chronic rheumatic mitral regurgitation (CRMR) was historically associated with predominant left ventricular (LV) dysfunction. The impact of left atrial (LA) dysfunction on morbidity and mortality has been minimised. Therefore, in a cross-sectional study we evaluated the atrioventricular mechanics in 77 patients with CRMR and 40 controls. LA and LV function were assessed by conventional echocardiography and 2D strain imaging. Mean age was $44 \pm 13.6$ years ( $83 \%$ female). LA stiffness index was greater in CRMR than controls $(0.95 \pm 1.89$ vs $0.16 \pm 0.13, p=0.009)$. LA dysfunction was noted in the reservoir and contractile phases compared to controls $(p<0.05)$. Conduit function parameters, except LA passive emptying fraction, were still preserved when compared to controls $(p<0.05)$. Peak LA strain in the reservoir phase $\left(\varepsilon_{p}\right)$, LA peak strain in the contractile phase $\left(\varepsilon_{C T}\right)$ and LV peak systolic strain (PSS) were decreased in CRMR compared to controls $(p<0.05)$. Eighty-six percent of the patients had decreased LA $\varepsilon_{R}$ and $58 \%$ had depressed LV PSS. Decreased $\varepsilon_{R}$ and normal LV PSS was noted in $42 \%$ of the patients. Thirteen percent had normal $\varepsilon_{R}$ and LV PSS. Only one patient had normal $\varepsilon_{R}$ with decreased LV PSS. On multivariate linear regression analysis, the main determinants of LA $\varepsilon_{R}$ were age, LV PSS and LA volume indexed $(p<0.001)$. In conclusion, LA dysfunction predominates in the reservoir and contractile phases in CRMR. LA dysfunction likely precedes LV dysfunction, potentially prompting earlier surgical intervention.

Key words: Chronic rheumatic, mitral, regurgitation

\section{CS/TH-P-42: INFECTIVE ENDOCARDITIS SECONDARY TO INTRAVENOUS DRUG ABUSE}

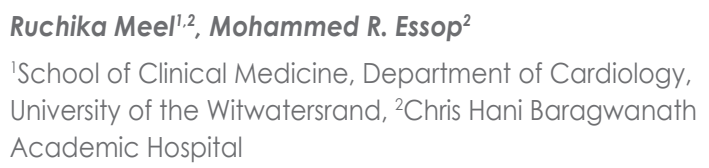

Infective endocarditis (IE) secondary to intravenous drug abuse (IVDA) is rare in the African population. We have recently described 3 cases of IE secondary to IV Nyaope (variable mixture of heroin, cannabis, antiretrovirals, metamphetamines) use. Since then we have encountered an additional 47 cases. Therefore, we aimed to prospectively document the clinical and echocardiographic characteristics of patients presenting at Chris Hani Baragwanath Academic Hospital with IE, secondary to IVDA. Mean age was 23 years (48 males). Majority presented with dyspnea $(88 \%)$, symptoms of withdrawal (30\%), peripheral suppurative infection (22\%) and right ventricular (RV) failure (14\%). Most were HIV reactive (88\%) and not on ARVs. Five patients had a CD4 count $\leq 200$ cells $/ \mu$ l. Renal failure requiring dialysis was present in 2 patients. Hepatitis $C$ antibody was detected in $58 \%$. Staphylococcus aureus was cultured in $68 \%$, pseudomonas aeruginosa in 1 patient and enterococcus faecalis in 3 patients. Polymicrobial infection was found in 6 patients. Sterile blood cultures were present in 8 patients. Tricuspid valve endocarditis was present in the majority (88\%). Mean vegetation size was $14 \mathrm{~mm}$ and $34 \%$ had pulmonary hypertension and RV dysfunction. Septic pulmonary emboli were documented in $40 \%$ of patients. All were treated with antimicrobials and only 4 patients underwent surgery. Three patients died secondary to respiratory failure. In conclusion, IE secondary to IVDA is on the rise among young black South Africans and is responsible for morbidity and mortality. A high degree of suspicion must be maintained ensure early diagnosis and treatment.

Key words: Infective endocarditis 


\section{CS/TH-P-43: RADICULAR CYST: ATYPICAL PRESENTATION AND THERAPEUTIC DILEMMA}

Saidah Tootla, Veloshnie Premviyasa

Department of Oral Rehabilitation, School of Oral Health Sciences, Faculty of Health Sciences, University of the

Witwatersrand, Johannesburg, South Africa

As more patients are presenting with cystic lesions within the South African setting, clinicians are required to perform endodontic treatment on multiple teeth. Radicular cysts most commonly occurs in men in the third to fifth decades of life and presents in the maxillary anterior region. These cysts are usually asymptomatic with noticeable swelling extra-orally. Although these lesions frequently heal simply with endodontic therapy alone, larger lesions may need additional treatment. When surgical enucleation with endodontics is the treatment of choice, there is a chance for inadvertent injury to adjacent teeth or structures. This case highlights an unusual presentation of symptomatic multilocular radicular cysts in the mandible of a female patient, subsequent root treatments of the involved teeth and surgical enucleation thereof. It seems apparent that radicular cysts should be treated on an individualised basis lending merit to the size, location and extent of the lesion.

Key words: Radicular cysts

\section{CS/TH-P-44: AN ECHOCARDIOGRAHIC COMPARISON OF ISOLATED LEFT VENTRICULAR NONCOMPACTION VERSUS IDIOPATHIC DILATED CARDIOMYOPATHY}

Nel Samantha ${ }^{2}$, Ferande Peters ${ }^{1,2}$, Elena Libhaber ${ }^{1}$, Mohammed R. Essop ${ }^{1,2}$, Claudia Dos Santos ${ }^{2}$, Hiral Matioda ${ }^{2}$

'School of Clinical Medicine, Echocardiography, Faculty of Health Sciences, University of the Witwatersrand, Johannesburg, South Africa, ${ }^{2}$ Department of Cardiology, Chris Hani Baragwanath Hospital, South Africa

An echocardiograhic comparison of isolated left ventricular noncompaction versus idiopathic dilated cardiomyopathy. Diagnosing isolated left ventricular noncompaction (ILVNC) is often challenging, as it is easily mistaken for idiopathic dilated cardiomyopathy (IDCM). The aim of this study was to characterise the functional and structural echocardiographic features, other than the established ILVNC criteria, that could be used to differentiate these two conditions. This prospective study evaluated 98 patients, 49 with ILVNC and 49 with IDCM. We utilised our previous published comprehensive echocardiographic criteria to identify ILVNC. IDCM was diagnosed where an ejection fraction was $\leq 45 \%$ and an end diastolic dimension of $>55$ $\mathrm{mm}$ was found on echo. The exclusion criteria for both ILNVC and IDCM were documented hypertension, known coronary artery disease, organic valvular disease, any systemic illness, or any primary organ failure. No significant differences were seen in the clinical characteristics and echocardiographic features of patients with ILVNC compared to their IDCM counterparts, with the exception of greater E-wave velocities in patients with ILVNC $(p=0.05)$. Apart from myocardial wall structure there were no differences in the functional parameters that allow for differentiation between ILVNC and IDCMO. It therefore remains important to carefully apply the echocardiographic criteria for ILVNC in order to accurately make the diagnosis.

Key words: Left ventricular noncompaction, idiopathic dilated cardiomyopathy

\section{CS/TH-P-45: ASSESSMENT OF ADHERENCE TO WARFARIN ANTICOAGULATION USING THE RATIO OF VITAMIN K DEPENDENT FACTORS}

Sarisha Naidoo, Johnny Mahlangu

School of Pathology, Department of Molecular Medicine and Haematology, Faculty of Health Sciences, University of the Witwatersrand, Johannesburg, South Africa

Adherence to warfarin has dual anticoagulant effect (reflecting the short half-life clotting factors (CF)) and antithrombotic effect (reflecting the long half-life of CF). The aim of this study was to evaluate adherence to warfarin therapy by measuring the factor VII (FVII) and factor II (FII) level in relation to therapeutic international normalised ratio (INR). This was a prospective cross sectional study in which participants were $\geq 18$ years, attendees of the anticoagulation clinic at Charlotte Maxeke Johannesburg Academic Hospital (CMJAH), on warfarin for $\geq 3$ months with a therapeutic INR. A $5 \mathrm{ml}$ venous sample was analysed for factor II and VII on a STA-R coagulation analyser. Of the 350 participants on warfarin for $\geq 3$ months, 92 had a sub-therapeutic INR, 45 were above therapeutic range and 213 had an INR in therapeutic range. Only two of the 204 patients had a therapeutic INR with either a low factor VII level or factor II level. Using a control group of 79 of patients with a sub therapeutic INR, sensitivity (i.e. true adherents) was $79.8 \%$ and specificity (i.e. true nonadherents) was $93.3 \%$. Non-adherence on steady state warfarin anticoagulation was very low and the ratio of vitamin $\mathrm{K}$ dependent factors proved to be less informative about patient adherence. While the INR is good test to detect non-adherent patients, it has shown to overcall adherent patients as being non-adherent. This may result in the inappropriate dose increases of warfarin with associated increase in bleeding risks.

Key words: Warfarin, INR, factors, adherence 


\section{CS/TH-P-46: HEAD AND NECK LYMPHOMA REVIEW: ORAL PATHOLOGY UNIT, SOUTH AFRICA}

Shabnum Meer, Nasreen Alli

Department of Pathology, School of Oral Health Sciences, Faculty of Health Sciences, University of the Witwatersrand, Johannesburg, South Africa

Non-Hodgkin lymphoma (NHL) occurs with increasing frequency in patients with HIV/AIDS. As South Africa has the highest incidence of HIV/AIDS worldwide, an epidemiologic study would provide insight into head and neck lymphomas in a defined South African population. This retrospective review evaluated the frequency and clinico-pathologic characteristics of patients diagnosed with head and neck lymphoma at the Oral Pathology Department, University of Witwatersrand between 19932012. Histopathology reports ( $n=22459$ ) of patients with head and neck lymphomas were reviewed. Demographic (age, gender), clinical (site and size of tumour), laboratory, and histological parameters were recorded. There were 504 patients $(2.24 \%)$ with head and neck lymphomas. The age ranged from 2 to 100 years (median: 39 years). The male:female ratio was 1.13:1. The cervical lymph node was the most common anatomic site (115 cases) and the maxilla (60 cases) the most common extranodal site. Plasmablastic lymphoma (159 cases) was the most common histologic subtype followed by diffuse large B-cell lymphoma (155 cases). The most common Hodgkin's lymphoma $(\mathrm{HL})$ was nodular sclerosing $\mathrm{HL}$ (21 cases). NHLs formed $90 \%$ of cases and $10 \%$ were HLs. Extranodal lymphomas represented $73 \%$ of cases, and $27 \%$ were nodal. There is an increase in head and neck lymphoma frequency, contrary to that found in Western countries, which show a decline with highly active antiretroviral therapy. Oral and maxillofacial lymphomas occurred predominantly in the third decade with a male bias. Most were extranodal, presenting as painful swellings. This study serves as a baseline for future studies, especially in South Africa.

Key words: Head and neck lymphomas

\section{CS/TH-P-47: PRUNE BELLY SYNDROME: A 30 YEAR EXPERIENCE AT THE CHRIS HANI BARAGWANATH ACADEMIC HOSPITAL, SOUTH AFRICA}

Shannon Leahy', K Petersen², U Kala²

'Department of Paediatrics, School of Clinical Medicine Faculty of Health Sciences, University of the Witwatersrand, Johannesburg, South Africa, ${ }^{2}$ Peadiatric Nephrologist

Prune Belly Syndrome (PBS) is a rare congenital disorder with a triad of signs: absent abdominal wall musculature, urinary tract malformations and cryptorchidism. The complete syndrome is seen in males. Management aims to prevent urinary sepsis and restore anatomy for medical and cosmetic reasons. Objectives of this study were to describe the patient profile, management, hospitalisation and outcome of patients attending the paediatric renal clinic at Chris Hani Baragwanath Academic hospital over 30 years. Methods: retrospective descriptive record review of patients with PBS. Results: 44 patient files were analysed. Median duration of follow up: 24 months (52 days-10.9 years). Median age at presentation was 5.5 days (1 day -2 years). Sixty six percent of patients were referred from another hospital. Associated conditions (urological 50\%; orthopaedic 11\%; congenital heart disease $7 \%$, gastrointestinal $4.5 \%$ ) were seen in $60 \%$ of patients. Sonar findings: $59 \%$ had hydronephrosis and $43 \%$ had hydroureter. Medical management comprised of UTI prophylactic antibiotics and intermittent bladder catheterisation. Surgical management included abdominoplasty, orchidopexy (median age three years) and circumcision (median age 7.7 years). The median UTI/year was one per patient, with $52 \%$ requiring inhospital treatment. Age of presentation and medical management did not have significant effects on renal outcome (reduced CGFR at last clinic visit). Default rate at the clinic was $57 \%$.

Conclusion: patient profile and management is comparable to other reports however associated conditions and ESRD was observed less in this review.

Key words: Prune, Belly, Syndrome

\section{CS/TH-P-48: INCREASED OPPORTUNITIES FOR ADVANCED THERAPIES IN HIGH RISK PAEDIATRIC MALIGNANCIES}

Tanya Schickerling ${ }^{1,2}$, Jennifer Geel'

'Department of Paediatric Oncology, School of Clinical Medicine, Faculty of Health Sciences, University of the Witwatersrand, Johannesburg, South Africa; ${ }^{2}$ Stem cell Transplant unit

In a country still grappling with a legacy of inequality, haemopoeitic stem cell transplant (HSCT) programmes have not been prioritised. In preparation for the opening of a cellular therapies unit at the Nelson Mandela Children's Hospital, an audit of past practices was conducted. A retrospective analysis of children undergoing HSCT at a state-academic and a private-academic hospital was performed. Descriptive statistics, Kaplan-Meier survival curves and Cox regression analysis were calculated. From January 1980 to December 2015, 39 stem cell transplants were performed on patients diagnosed in our unit, including 19 autologous and 20 allogeneic transplants. The majority of patients (31/39) came from Gauteng Province. Median age was 4.6 years (range 2.8 months to 16 years). Indications for transplant included haematolymphoid malignancies (13), solid tumours (15) and non-malignant haematological conditions (11). Twenty-three procedures were performed in our combined unit while 16 patients 
were referred elsewhere. Sixteen patients (41\%) are alive and disease-free, four are alive with disease, 15 died from relapsed disease and four (10.3\%) died of treatmentrelated mortality (TRM). Three patients developed second malignant neoplasms, one of whom demised. The median follow-up period was 624 days (range 44 days to 15.6 years). Five year overall survival (OS) from transplant was $52.7 \%$ with no difference detected between indication for transplant $(p=0.79)$ or geographical location of transplant $(p=0.38)$. Limited resources and expertise have resulted in poor access to a life-saving treatment modality. Implementation of a dedicated HSCT programme will increase access for children with high risk malignancies, both locally and country-wide.

Key words: Stem cell transplant, Paediatrics

\section{CS/TH-P-49: POOR PROGNOSTIC FEATURES AND METASTATIC DISEASE AT PRESENTATION CONTRIBUTE TO LOW SURVIVAL RATE OF CHILDREN WITH OSTEOGENIC SARCOMA IN JOHANNESBURG}

Thandeka Ngcana, TVZ Ngcana, J Geel, J Poole

Department of Paediatrics and child health, School of

Clinical Medicine, Faculty of Health Sciences, University of the Witwatersrand, Johannesburg, South Africa

Survival rates of South African children with osteogenic sarcoma (OS) are known to be poor, ranging from $7.5 \%$ to $30 \%$. An audit was performed at Charlotte Maxeke Johannesburg Academic Hospital (CMJAH) to assess the prognostic features and survival rates in patients treated at a single centre. A retrospective folder review was conducted of all children with documented OS at CMJAH over a 20 year period. Sixty four patients with histologically proven OS were identified, representing $3.3 \%$ of the total paediatric malignancies treated in the hospital during the study period. The ratio of male to female was 1:1 and the majority of patients (40/55) were of African ethnicity. The mean duration of symptoms was 90 days. Most patients presented with poor prognostic features: 47 (85\%) had masses larger than 8cm diameter; 55 (100\%) presented with high alkaline phosphatase and 46 (83.6\%) had elevated lactate dehydrogenase (LDH). Histology included osteoblastic (37/55) and chondroblastic OS $(24 / 55)$. Five out of fifty five of these were telangiactatic subtype. Thirty five of the patients were underweight for age at presentation. Forty one patients were treated with chemotherapy. The intent-to-treat five year survival rate was $42 \%$. Causes of death included disease (22/41) and treatment complications (5/41). The following factors were found to impact significantly on survival: nutrition $(p=0.0495)$, elevated LDH $(p=0.01)$, stage (0.048), response to chemotherapy $(p=0.05)$ and metastatic disease at presentation $(p=0.0028)$. The dismal survival rate of this group of patients, while higher than that reported in other South African series, may be improved by increased attention to modifiable factors such as nutrition and diagnostic lag time. Delayed presentation is common in the South African setting; lag time is longer than in high income countries.

Key words: five year survival rate, Ostesarcoma, children

\section{CS/TH-P-50: BILATERAL TRAUMATIC POSTERIOR HIP FRACTURE DISLOCATIONS: A REVIEW OF 2 CASES}

Thando Ncube', Mohammed Nasir lqbal', Mmampapatla Thomas Ramokgopa ${ }^{4}$

'Department of Orthopaedic Surgery, School of Clinical Medicine ${ }^{2}$ Orthopaedic Registrar Chris Hani Baragwanath Hospital, ${ }^{3}$ Consultant Orthopaedic Surgeon, Chris Hani Baragwanath Hospital, ${ }^{4}$ Head Orthopaedic Surgery, Chris Hani Baragwanath Hospital

Hip dislocations result from high energy injuries with posterior dislocations predominating. Posterior hip dislocation usually arises from an anteroposteriorly directed force on a flexed knee during motor vehicle accidents. Due to the high energy involved there may be associated musculoskeletal and visceral injuries. Bilateral traumatic posterior hip dislocations are uncommon with few cases being reported in literature. We present two cases of this unusual condition and discuss its pathoanatomy, management and outcome. A 28 year old female was involved in an MVA and sustained bilateral hip dislocations, open book pelvis, acetabular fractures and humerus fracture. Left humerus intramedullary nail was done. Delayed hip reduction and staged pelvic reconstruction was also done. Sciatic nerve palsy recovered gradually but the patient presented later with left posterior hip dislocation which was planned for total hip replacement. The second patient is a 30 year old female involved in an MVA. She sustained bilateral posterior hip dislocations, bilateral superior and inferior pubic rami fractures and a left femur fracture. Emergent closed hip reduction and intramedullary nailing of the femur were done. Elective reconstruction of right acetabulum was done and the left acetabulum was treated conservatively. Post-operatively the patient complicated with sciatic nerve palsy which recovered gradually.

Conclusion: We postulate that the severe axial load on the hip joints rips open the pelvis anteriorly. The resulting injury is either symphiseal diastasis or fracture of the ischiopubic bones. Aggressive resuscitation and urgent hip reduction is warranted in order to obtain good outcome. Pelvic reconstruction may be delayed until the patient is stable. Sciatic nerve palsy complicates posterior hip dislocation as well as acetabular surgery and recovery is slow.

Key words: Bilateral, fracture, hip dislocations 


\section{CS/TH-P-51: PREDICTORS OF OCCURRENCE OF OMENTAL DEPOSITS IN PATIENTS WITH GASTRIC ADENOCARCINOMA UNDERGOING GASTRECTOMY: A HISTOPATHOLOGICAL AUDIT}

Thifheli Luvhengo', Odimae Tobiko², Christine Jann-Kruger², Joshua Kiluba 3 , Martin Hale 4

'School of Clinical Medicine, Faculty of Health Sciences, University of the Witwatersrand: ${ }^{2}$ Department of Surgery, University of the Witwatersrand, ${ }^{3}$ Faculty of Health Sciences, University of the Witwatersrand, Johannesburg, South Africa, ${ }^{4}$ Department of Anatomical Pathology, University of the Witwatersrand, NHLS

The milky spots of greater omentum are primary sites for seeding of exfoliated cells from a transmural gastric cancer. Surgery is the mainstay of treatment of gastric adenocarcinoma and the greater omentum is usually resected en-bloc. The aim of this study was to determine if pathologists routinely perform histopathological analysis of greater omentum and factors associated with findings of cancer deposits. An audit of histopathology records of patients who had gastric malignancies between 2008 and 2012 was undertaken. Data retrieved included patients' demography, tumour site, tumour histology and subtypes, Helicobacter pylori status, associated gastritis, types of gastrectomy and, analysis and finding of omental deposits. Records of 325 patients were found. Majority (76.6\%) were adenocarcinomas and there were only few cases of GISTs and lymphoma. The overall male to female ratio of patients was 192:133 and their average age overall was 59.0 years (range: 23-102 years). The average age of patients who had adenocarcinoma was 60.2 years. About $8.8 \%$ of patients who had adenocarcinoma were younger than 40 years. Gastric resection was performed in $23.1 \%$ adenocarcinomas of which $9.1 \%$ was stage I. The greater omentum was part of specimen in $46.6 \%$ cases but report regarding cancer deposits was specified in $25.9 \%$ of which $11.1 \%$ were positive. Pathologists do not routinely analyse and report findings on the greater omentum of patients who had gastrectomy for cancer. It potential leads to understaging. Assessment of greater omentum should be incorporated into the standard pathology reporting format for advanced gastric cancer.

Key words: Gastric cancer, greater omentum, milky spots, omental deposits

\section{CS/TH-P-52: PREVALENCE, DEMOGRAPHIC AND HISTOLOGICAL SUBTYPES OF HURTHLE CELL TUMOURS OF THE THYROID: A HISTOPATHOLOGICAL AUDIT}

Victor Malith, Harran N, Bombil I, Luvhengo TE

Department of Surgery, School of Clinical Medicine, Faculty of Health Sciences, University of the Witwatersrand, Johannesburg, South Africa
Hurthle cell neoplasms (HCNs) account for 3-10\% of thyroid carcinomas and adenomas. The aim of this study was to determine factors associated with malignancy in patients who had thyroidectomy for HCNs at University of Witwatersrand teaching hospitals. Histopathology records of patients who had thyroidectomy from January 2001 to October 2015 were reviewed. Data retrieved included indications for thyroidectomy, demographics, $\mathrm{HCN}$ subtypes and pre-operative FNAC results. At total of 2641 records were found and 676 were for thyroid tumours. Around 107 cases were $\mathrm{HCNs}$ and $25.2 \%$ of HCNs were HCCs. Average tumour size for FCAs and FCC were $4.9 \mathrm{~cm}$ and $3.5 \mathrm{~cm}$ respectively, and was statistically significant $(p=0.016)$. The risk of malignancy increased from $11.1 \%$ in FCNs less or equal to $1 \mathrm{~cm}$, through $33.3 \%$ for size of $1-4 \mathrm{~cm}$ to $53.8 \%$ for tumour larger than $4 \mathrm{~cm}$. Pre-operative FNAC results were available in $54.2 \%(58 / 107)$ and were reported as follows: suspicious for $\mathrm{HCNs}=31$, suspicious of papillary carcinoma $=7$, atypia of unknown significance $=5$, benign $=6$ and non-diagnostic $=6$. Age and gender were not significant demographical factors predictable for malignancy. $\mathrm{HCC}$ is relatively more common in parts of South Africa than it is reported. A HCN larger than $4 \mathrm{~cm}$ in diameter is likely to be HCC. Therefore total thyroidectomy should be considered in patients presenting with thyroid nodule greater than $4 \mathrm{~cm}$ diameter if FNAC result suggests $\mathrm{HCN}$.

Key words: Hurthle cell neoplasms, Hurthle cell carcinoma, FNAC, Tumour size

\section{CS/TH-P-53: HIV PREVALENCE AND MORBIDITY IN OLDER MEDICAL IN- PATIENTS: DATA FROM A HIGH HIV PREVALENCE SETTING.}

Vivendra Naidoo, Neil Martinson

School of Clinical Medicine, Department of Internal Medicine, Perinatal HIV Research Unit, Faculty of Health Sciences, University of the Witwatersrand, Johannesburg, South Africa

Current understanding of the burden of HIV infection and its serious comorbidity in older adults is limited, especially in South Africa. With the advent of ART, HIV-infected patients have survived to their sixth decade of life and beyond. Moreover, older individuals are not immune to new infection with HIV. South Africa has a high burden of non-infectious chronic diseases, especially hypertension and type 2 diabetes mellitus (T2DM). This study aims to determine the demographics and disease profile of patients aged 50 years and over. Patients aged 50 years and older admitted to Tshepong hospital medical wards were approached to participate. After informed consent was obtained, socio-demographic, anthropomorphic, admission diagnoses and HIV serostatus were collected. Overall 151 participants were enrolled; their mean age was 62.3 years (standard deviation 8.83) and 88/151 (59.5\%) were women. Forty-eight of the 151 participants were HIV-infected (31.8\%). Among HIV-infected participants, 
37/48 (77.1\%) were known to be HIV infected, their mean age of HIV diagnosis was 53.8 years $(41-72)$; 11/48 HIVinfected individuals were diagnosed during recruitment admission and a total of 20/48 were diagnosed within the last year. The most common co-morbidities amongst the subset of patients were hypertension and T2DM. None of the newly diagnosed HIV-infected patients presented with HIV related diseases. Four of the known HIV-infected patients were admitted with a provisional diagnosis of HIV related infections. Our study demonstrates that in the ART era, older patients have similar age-related non-infectious chronic illnesses, irrespective of HIV serostatus and are also at risk for new HIV infection.

Key words: HIV, Chronic illnesses

\section{CS/TH-P-54: AN ARTERIOVENOUS MALFORMATION IN THE PRESENCE OF A PERSISTENT TRIGEMINAL ARTERY}

Wilheminah Makhambeni ${ }^{1,2}$, Victor Mngomezulu², Werner van Straaten $^{2}$

Department of Neurosciences, School of Clinical Medicine, Faculty of Health Sciences, University of the Witwatersrand, Johannesburg, South Africa

A pathological insult in the presence of architectural abnormality influences the interpretation of the condition's pathophysiology. A persistent trigeminal artery (PTA) occurs at a frequency of $0.03-2.2 \%$ with a female predilection. A PTA may be associated with anomalies of the internal carotid artery - a complete agenesis or segmental regression of the (cavernous and/or cervical) - and/or the basilar artery (non-fusion; segmental hypoplasia/agenesis). Although there are case reports of arteriovenous malformations (AVM) in the presence of PTAs, they are still so rare that Yasargil found none in a 500 AVM case series. We report on the only reported case of a thalamomesencephalic arteriovenous malformation in the presence of a Saltzman type 2 ipsilateral posterior communicating artery agenesis - PTA. A 27 year old male patient, with no prior neurological deficit or chronic disease, suddenly collapsed. Upon recovery of consciousness, he had confusion and a left hemiparesis. He was normotensive. A CT scan revealed a haemorrhagic right thalamic AVM. A subsequent MRI showed midbrain involvement of the $3 \mathrm{~cm}$ AVM. The left vertebral artery run of a digital subtraction angiogram confirmed a compact nidus, supplied by branches of medial basilar tip perforators, and a single superficial vein drained into the right internal jugular vein. The right internal carotid angiogram revealed a Saltzmann type 2 PTA (absent right posterior communicating artery). No overt bleeding point was found, and the risk of death/ worse disability due to surgery/endovascular intervention was deemed unacceptable. The fact that no other cases were previously published emphasised the importance of understanding the anatomy and vascular supply of the perilesional vital structures.
Key words: Persistent trigeminal artery, arteriovenous malformation

\section{CS/TH-P-55: ANALYTICAL PERFORMANCE OF THE NIJMEGEN ASSAY USING DIFFERENT BUFFERED REAGENTS}

Yuen On Wan, Johnny Mahlangu

Department of Molecular Medicine and Haematology, School of Pathology, Faculty of Health Sciences, University of the Witwatersrand and National Health Laboratory Service, Johannesburg, South Africa

Factor VIII Inhibitor measurement is important in the diagnosis and surveillance of Haemophilia patients. At low inhibitor values, the Nijmegen assay (NA) has better reliability compared with the Bethesda assay (BA). Reliability of the BA and NA is partially dependent on effectively buffered reagents such as normal pooled plasma (NPP) and Ci-Trol (Dade® Citrol $1 \circledR$ Coagulation Control Level 1). This study compared the use of NPP with Ci-Trol in the NA for inhibitor measurement. The optimization of the NA was as described by Verbruggen in 2014. The NPP and Ci-Trol were buffered with imidazole. Using either NPP or Ci-Trol, the NA was performed on control plasmas with assigned values and known inhibitor patient plasmas. Of the 100 patient plasmas, 100 were analysed with NPP and 43 with Ci-Trol. In the NA using NPP, the control plasma coefficient of variation (CV) was $8.44 \%$ (95\% confidence interval (Cl) of $0.77 \pm 0.05$ ) with a standard deviation (SD) of 0.06 . In the NA using Ci-Trol, the control plasma CV was $10 \%(95 \% \mathrm{Cl}$ of $0.93 \pm 0.02$ ) and SD was 0.09 . In the Bland-Altman plot of NA vs BA using the NPP, the bias was $0.49(95 \% \mathrm{Cl}$ of -8.1 to 9.1). The bias between NA and BA using Ci-Trol was 0.8 (95\% Cl of -6.8 to 8.5$)$. The correlation coefficient of NA vs BA using NPP was 0.93 and that using Ci-Trol was 0.76 . All control plasma analyses using NPP were within reference values whilst only 2 of 3 values were within the assigned values using $\mathrm{Ci}$-Trol. In this study, the performance of the Nijmegen assay with buffered pool plasma was better than that using buffered Ci-Trol.

Key words: Haemophilia, inhibitor, diagnosis

\section{CS/TH-P-56: A COMPARISON OF SEDENTARY BEHAVIOUR IN PATIENTS WITH KNEE OSTEOARTHRITIS COMPARED TO HEALTHY CONTROLS}

Yusuf Suleiman Kaoje', Joanne Alexandra McVeigh², Rebecca Mary Meiring'

'School of Physiology, Exercise Laboratory Unit, Faculty of Health Sciences, University of the Witwatersrand, Johannesburg, South Africa, ${ }^{2} S c h o o l$ of Occupational therapy and social work, Curtin University, Perth, Western Australia.

It is known that physical activity (PA) is beneficial in patients with knee osteoarthritis (KOA). Detailed, objective data on sedentary behaviour (SB) patterns will therefore also help to inform targeted interventions in people with 
KOA. The purpose of this study was to measure patterns of $S B$ in patients with end stage KOA and to compare these patterns to a healthy age matched control group (CON). Participants (CON $n=15 ; O A n=24)$ were males and females between the ages of 50 and 85 years. SB was measured for seven days for 24 hours a day using an activPAL accelerometer in patients diagnosed with KOA and in age matched control participants. Sleep time, recorded using a sleep questionnaire, was removed in order to calculate wear time for each participant. Total daily time spent in SB was significantly greater in patients with OA (587.8 $\pm 183.9 \mathrm{~min} /$ day) compared to CON (494.0 $\pm 46.2 \mathrm{~min} /$ day; $\mathrm{p}=0.002$ ). Time spent in upright activities tended to be greater in the CON (496.4 $\pm 92.9 \mathrm{~min} /$ day) than in the OA patients $(354.8 \pm 143.7 \mathrm{~min} /$ day; $p=0.053)$. There was no difference in the number of breaks in sedentary time per day between the groups (CON: $50.7 \pm$ 15.9 breaks, OA: $42.9 \pm 14.2$ breaks; $p=0.566$ ). Patients with KOA spent a greater amount of time in SB and less time in upright activities compared to a healthy control group. The fact that there were no differences in the number of breaks in sedentary time leads us to believe that further investigation into the patterns of sedentary behaviour is required in this patient population.

Key words: Knee osteoarthritis, sedentary behaviour, patterns, activPAL 


\section{DISEASES OF LIFESTYLE}

\section{DL-O-1: INTRA-FAMILIAL AGGREGATION AND HERITABILITY OF TISSUE DOPPLER INDEXES OF LEFT VENTRICULAR DIASTOLIC FUNCTION IN A GROUP OF AFRICAN DESCENT.}

Vernice Petersen', Gavin Norton', Carlos Libhaber², Muzi Maseko', Pinhas Sareli', Angela Woodiwiss'

'School of Physiology, Cardiovascular Pathophysiology and Genomics Research Unit, University of the Witwatersrand, ${ }^{2}$ School of Clinical Medicine, Faculty of Health Sciences, University of the Witwatersrand

There are limited proven therapies for heart failure with a preserved ejection fraction (associated with a reduced diastolic function) and hence identifying the mechanisms responsible is of importance. Although several indexes of left ventricular (LV) diastolic function show heritability, the genetic influence on the tissue Doppler index, E/e' (early transmitral velocity/velocity of myocardial tissue lengthening), an index of LV filling pressures in those of black African descent is currently unknown. Intra-familial aggregation and heritability (SAGE software) of $E / e^{\prime}$ (echocardiography) were assessed in 129 nuclear families (29 spouse pairs, 216 parent-child pairs and 113 siblingsibling pairs) from an urban developing community of black Africans. Independent of confounders including LV mass index (LVMI) and relative wall thickness (RWT), E/e' was correlated in parent-child $(r=0.23, p<0.001)$ and sibling-sibling $(r=0.29, p<0.005)$, but not in spouse $(r=0.13$, $p=0.51$ ) pairs. The relationships between parent-child and sibling-sibling pairs persisted with adjustments for aortic pressure. The relationships between parent-child $(r=0.22$, $p<0.001)$ and sibling-sibling $(r=0.26, p<0.01)$ pairs also persisted with adjustments for backward wave pressures (Pb). Independent of confounders including LVMI and RWT, E/e' showed significant heritability (h2 \pm SEM $=0.51 \pm 0.11$, $p<0.0001)$ which similarly persisted with adjustments for aortic pressure. In conclusion, in a group of African ancestry, independent of LV remodeling and aortic function, E/e', shows significant intra-familial aggregation and robust heritability. Hence, genetic factors may play an important role in determining moderate-to-severe LV diastolic dysfunction in groups of black African ancestry.
Key words: Left ventricular diastolic function, heritability, aortic function

\section{DL-O-2: VITAMIN D LEVELS OF ANAESTHETISTS IN THE DEPARTMENT OF ANAESTHESIOLOGY AT THE UNIVERSITY OF THE WITWATERSRAND}

Eugene Kelly, Helen Perrie, Juan Scribante, Sean Chetty

School of Clinical Medicine, Department of Anaesthesiology, University of the Witwatersrand

There has been a resurgence of interest in vitamin $D$ and its effects. Theatre personnel should be cognisant of vitamin $D$ deficiency as an occupational hazard, as it is a global problem that has been studied extensively in colder climates. No research was identified among medical personnel in South Africa. The primary objective of this study was to describe serum 25(OH)D (25-hydroxyvitamin D) levels of anaesthetists. The secondary objective was to describe and compare factors influencing vitamin D levels in anaesthetists who are vitamin D insufficient to those who are not. Data was collected in winter 2013. Anaesthetists agreeing to participate signed the informed consent. They then completed the questionnaire comprising demographics and secondary objectives. Each participant had $5 \mathrm{ml}$ of blood collected into an EDTA specimen tube. The samples were processed by qualified laboratory personnel using High Performance Liquid Chromatography determine 25(OH)D levels using a Shimadzu ${ }^{\circledR}$ Ultra performance liquid chromatography system. The median $25(\mathrm{OH}) \mathrm{D}$ was $43.8 \mathrm{nmol} / \mathrm{l}$ (IQR 26-76), with 51 of 89 (57.30\%) anaesthetists being vitamin $D$ insufficient. There was a statistically significant association between ethnicity and vitamin D status ( $p<0.001) .21$ (80.77\%) Indian anaesthetists and $14(70.00 \%)$ black anaesthetists were insufficient, as compared to only $10(28.57 \%)$ white anaesthetists. Vitamin $D$ is no longer a forgotten vitamin. Anaesthetists should be considered at risk for vitamin $D$ deficiency. Adequate vitamin D levels should be seen as essential rather than optional even in "sunny" climates.

Key words: Vitamin D, deficiency, anesthesiolgy 


\section{DL-O-3: REFLECTED WAVES OBTAINED USING A TRIANGULAR FLOW WAVE METHOD ARE AS CLOSELY RELATED TO LEFT VENTRICULAR MASS AS THOSE OBTAINED USING ECHOCARDIOGRAPHIC DERIVED AORTIC FLOW WAVES}

Imraan Ballim, Angela Woodiwiss, Hendrik Booysen, Andrew Raymond, Gavin Norton

School of Physiology, Cardiovascular Pathophysiology and Genomics Research Unit, Faculty of Health Sciences, University of the Witwatersrand

Aortic backward (reflected) waves are major determinants of cardiovascular events and their impact is independent of brachial blood pressure. Although aortic backward wave pressures $(\mathrm{Pb})$ can be determined using a triangular flow wave form for wave separation analysis, $\mathrm{Pb}$ derived from this approach correlates poorly with $\mathrm{Pb}$ derived from measured aortic flow waves. However, the comparative ability of these two $\mathrm{Pb}$ measurements to predict endorgan changes remains uncertain. Therefore, we aimed to compare $\mathrm{Pb}$ obtained using a triangular flow wave method (Pbtri) and Pb obtained using echocardiographic derived aortic flow velocity waves (Pbecho), and their relationships with left ventricular mass index (LVMI). In 300 participants from a black African community sample (age>16years), aortic haemodynamics (applanation tonometry, SphygmoCor software), aortic flow velocity and LVMI (echocardiography) were determined. BlandAltman analysis revealed that Pbtri overestimated the backward wave pressure by an average of $3.62 \pm 3.17 \mathrm{mmHg}$. However, the correlation between the two measurements was markedly high ( $\mathrm{r} 2=0.88)$. Independent of confounders, Pbtri was associated with LVMI (partial $r=0.21, p<0.0001$ ). Importantly, when comparing the association between Pbecho and LVMI (partial $r=0.24, p<0.0001$ ) no differences were noted ( $p=0.35$, for comparison of partial $r$ values, $z$ score). In conclusion, a triangular flow wave form employed for wave separation analysis produces $\mathrm{Pb}$ values that are as closely associated with LVMI as those derived from echocardiographic aortic flow wave measurements.

Key words: Backward wave pressures, Triangular flow wave, Left ventricular mass

\section{DL-O-4: THE RELATIONSHIP BETWEEN PHYSICAL FUNCTION AND DISEASE ACTIVITY IN RHEUMATOID ARTHRITIS: RESULTS FROM THE METEOR DATABASE}

Lai Ling Winchow ${ }^{1,2}$, Nimmisha Govind ${ }^{2}$, Eustasius Musenge ${ }^{2}$, Tom Huizinga ${ }^{2}$, Mohammed Tikly ${ }^{2}$

'School of Clinical Medicine, Department of Internal Medicine, 2Department of Rheumatology, Chris Hani Baragwanath Academic Hospital

The Health Assessment Questionnaire Disability Index (HAQ-DI) is the most widely used disease-specific measure of physical disability in rheumatoid arthritis (RA). In this study we investigated the response to standard of care treatment based on the HAQ-DI and EULAR response criteria in a cohort of established RA patients.421 active RA patients with complete HAQ-DI scores and disease activity scores were selected from the International Measurement of Efficacy of Treatment in the Era of Outcome in Rheumatology (METEOR) database. Cumulative probability curves were created to visualize the change in HAQ-DI between the different response groups. The proportions of patients achieving a Minimally Clinically Important Difference (MCID) of 0.22 improvements or deterioration of the HAQ-DI were calculated. The cohort showed the typical RA female predominance; had very active disease and moderate functional disability. As a group the patients showed an improvement in all variables of disease activity and HAQ-DI scores after a mean period of 8.7 months. When applying the EULAR response criteria, $47 \%$ achieved a $\mathrm{good} /$ moderate response and the remainder had a poor response. The proportions of patients achieving a clinically important improvement, deterioration and no change of the HAQ-DI scores were $36 \%, 13 \%$ and $51 \%$ respectively. In this cohort of patients with established RA and high disease activity, less than $50 \%$ of patients achieved a EULAR good/ moderate response. In spite of this, the majority of patients (87\%) showed no change or had an improvement in physical function according to the HAQ-DI.

Key words: Rheumatoid Arthritis, Functional Disability

\section{DL-O-5: HEALTH AND AGEING IN AFRICA: LONGITUDINAL STUDIES OF AN INDEPTH COMMUNITY (HAALSI)}

Stephen Tollman', Stephen Tollman², Xavier Gomez-Olive², Chodziwadziwa Kabudula ${ }^{2}$, Fezile Mdluli2, Ryan Wagner ${ }^{2}$, Alisha Wade $^{2}$, Kathleen Kahn ${ }^{2}$, Lisa Berkman ${ }^{2,3}$

'School of Public Health, Rural Public Health and Health Transitions Research Unit (Agincourt), Faculty of Health Sciences, ${ }^{2}$ Wits, ${ }^{3}$ Harvard

Worldwide, by 2050 , adults $65+$ will outnumber children under-5 by a factor of 2.5. Yet evidence on the health, care and support of older persons is minimal. HAALSI, a collaboration of the Agincourt Unit, INDEPTH Network and Harvard University launched in South Africa in 2014. Focused on successful ageing in rapidly transitioning African contexts, we established a randomly selected cohort of 5,000 participants 40 years and over to address the trajectories of chronic disease and risk, their social, biologic and economic determinants, consequences for physical and cognitive function, and socioeconomic impacts. Fieldwork was conducted in the Agincourt sub-district, supported by a robust health and sociodemographic surveillance system (pop. 116,000); with pilot studies in Navrongo, Ghana and Ifakara, Tanzania. 
Features include follow-up of labour migrants, tabletbased cognitive assessments, and serology for HIV status and ART adherence; samples for genomic analyses involve a Wits-H3A Collaborating Centre. The study will support validation/correlation assessments between dry blood spot and venous markers, as well as between tabletbased and standard cognitive tests. Baseline findings on disease outcomes, risks and behaviours associated with cardiometabolic disease, HIV/AIDS and cognitive function among older adults in rural SA will be presented. Prior work suggests that poor self-reported health correlates with subsequent death. Mortality and cause-of-death trends among elders in sub-Saharan Africa, using publicly accessible data from the INDEPTH iShare repository, will also be reviewed. Further survey waves are planned for 2017-18 and 2020-21; in time we expect work to inform clinical, health and social policy development.

Key words: Ageing, chronic, health-transition, rural

\section{DL-O-6: OMENTIN CONCENTRATIONS ARE INDEPENDENTLY ASSOCIATED WITH THOSE OF MATRIX METALLOPROTEINASE-3 IN PATIENTS WITH MILD BUT NOT SEVERE RHEUMATOID ARTHRITIS}

Chanel Robinson 1,2, Linda Tsang 1,2, Ahmed Solomon ${ }^{3}$, Angela J Woodiwiss ${ }^{1,2}$, Sule Gunter ${ }^{2}$, Aletta E Millen², Gavin R Norton', Ivana Hollan ${ }^{4}$, Patrick HM Dessein ${ }^{2,4,5}$

'School of Physiology, University of the Witwatersrand, ${ }^{2}$ Cardiovascular Pathophysiology and Genomics Research Unit, University of the Witwatersrand, '3Department of Rheumatology, CMJAH, ${ }^{4}$ Vrije Universiteit Brussel, Belgium, ${ }^{5}$ Rheumatology Division Universitair Hospital Brussel

Adipose tissue derived adipokines are determinants of systemic metabolism and may contribute to cardiovascular pathology. We investigated the associations of omentin concentrations with subclinical cardiovascular disease in patients with rheumatoid arthritis (RA). Omentin concentrations were measured in 213 (104 black; 109 white) RA patients. Relationships of omentin levels with endothelial activation markers, carotid intima-media thickness and plaque, and matrix metalloproteinase (MMP)-2, -3 and -9 were identified in multivariate regression models. Omentin concentrations were inversely associated with MMP-3 levels $(\beta=-364$ (0.113), $p=0.002$ ). In stratified analysis, the omentin-MMP-3 concentration relationship was reproduced in white $(\beta$ (SE) $=-0.450$ (0.153), $p=0.0004)$ but not black patients $(\beta(S E)=-0.099(0.195), p=0.6)$, in participants with disease remission or mild disease activity $(\beta(S E)=-0.411$ (0.139), $p=0.004)$ but not with moderate or severe RA activity $(\beta(S E)=-0.286(0.202), p=0.2)$, and in those with a small $(\beta$ (SE) $=-0.534(0.161), p=0.001)$ but not large erythrocyte sedimentation rate (ESR) $(-0.212 \quad(0.168), p=0.2)$ and without $(\beta$ (SE) $=-0.554$ (0.165), $p=0.0001$ ) but not with large joint deformity counts $(-0.110(0.173), p=0.5)$. Omentin levels were unrelated to endothelial activation and atherosclerosis in these patients. Omentin concentrations were inversely associated with those of MMP-3 - a surrogate marker of plaque vulnerability to rupture - in white but not black Africans with RA. This inverse relationship was absent RA patients with moderate or severe RA activity and large ESR values and joint deformity counts. A loss of beneficial effects of omentin on plaque instability may contribute to the link between severe disease and increased cardiovascular risk in RA.

Key words: Omentin, Rheumatoid, Arthritis, Disease

\section{DL-O-7: PROFILING OF THE HUMAN CELL- CYCLE RELATED GENES' EXPRESSION IN HUMAN NON-SMALL CELL CARCINOMA TREATED WITH EFAVIRENZ AND LOPINAVIR/RITONAVIR}

Rahaba Marima', Clement Penny ${ }^{2}$

'School of Clinical Medicine, Department of Internal Medicine, University of the Witwatersrand, Medical Oncology, ${ }^{2}$ University of the Witwatersrand

South Africa has the largest ARV treatment programme in the world, wherein highly active antiretroviral treatment (HAART) has improved the quality of life in HIV/AIDS patients. On the contrary, cancers not previously associated with HIV/AIDS (non-Aids defining cancers; NADCS) have been shown to be increasing, compared to the AIDS defining cancers (ADCs). Lung cancer, as a NADC has been documented in the HIV/AIDS population. The poor understanding of the association between ARV drugs and lung cancer places a burden on public health, both globally and in SA. This study aimed to investigate the effects of Efavirenz and Lopinavir/ritonavir at plasma relevant levels in in-vitro lung cancer models. Since the deregulation of the cell cycle is a hallmark of lung cancer, the effects of EFV and LPV/r were screened on an arrayed panel of human cell cycle genes in the MRC-5 (lung epithelial cell line) and A549 (non-small cell lung cancer) lung cell-lines. Significantly expressed targets were further quantified and confirmed by RT-qPCR. Bio-informatics analysis (using STRING and Reactome) was done to further investigate potential interaction/s between the identified targets, and to determine novel target involvement in related biological pathways. Importantly, two identified targets, CASP3 and AURKB were shown to be either up or down-regulated, functioning at key cell cycle checkpoints and in cell cycle arrest. These findings demonstrate an effect of EFV and LPV/r on cell cycle related genes, acting to stimulate cell proliferation and thus suggesting their role in lung cancer.

Key words: HAART, Lung cancer, Cell cycle 


\section{DL-0-8:EARLY GROWTH AND BLOOD PRESSURE TRAJECTORIES IN URBAN SOUTH AFRICAN CHILDREN: BIRTH TO TWENTY COHORT}

Juliana Kagura', MSC (Med), Linda S Adair 1,2, Richard J. Munthali', John M Pettifor ' ${ }^{1}, \mathrm{MBBCh}$, Shane A. Norris ${ }^{1}$

'School of Clinical Medicine, Department of Paediatrics, Developmental Pathways for Health Research Unit, University of the Witwatersrand, ${ }^{2}$ University of North Carolina, ${ }^{3}$ University of the Witwatersrand

Early life growth has been shown to be associated with cross-sectional blood pressure (BP) values, but whether or not early life growth patterns are associated with longitudinal BP trajectories is under-researched. Therefore, we sought to examine the association between early growth and BP trajectories from childhood to adulthood. Data were utilized from the Birth to Twenty Plus cohort, from which black South African children ( $n=1937,52 \%$ girls) were selected for the analytical sample. BP was measured on seven occasions between ages 5 and 18 years and conditional variables for growth in infancy and mid-childhood were computed from anthropometric measures. We used a group-based trajectory modeling approach to identify distinct BP trajectories and then tested their association with growth between birth and mid-childhood unadjusted and adjusting for several covariates. Three sex-specific trajectory groups were identified for systolic BP (SBP) and diastolic BP (DBP): 'lower', 'middle' and 'upper' in both boys and girls. Height between 5 and 18 years was positively associated with SBP trajectories in boys and girls. In the final adjusted models taking into account height, prominent predictors in boys of the middle and upper SBP trajectories in comparison to the lower trajectory were birth weight lodds ratio [OR] $0.75[95 \% \mathrm{Cl} 0.58-0.96])$ and relative weight gain in infancy (4.11[1.25-13.51]). In girls, greater relative weight gain and relative linear growth in both infancy and midchildhood were consistently associated with an almost 2 -fold increase in OR of being in the upper versus lower SBP trajectory. Distinct trajectories of BP were identified with the upper trajectory potentially indicating greater risk for adult hypertension. Targeted early life interventions to address greater relative weight gain may be able to lessen the odds of being in a higher BP trajectory in late adolescence and early adulthood.

Key words: BP trajectories, weight gain, linear growth

\section{DL-O-9: EFFECTS OF URSOLIC ACID ON METABOLIC PROGRAMMING OF DIET- INDUCED METABOLIC DYSFUNCTION IN SPRAGUE DAWLEY RATS}

Nyasha Mukonowenzou 1,2, Nyasha Mukonowenzou 1,3, Eliton Chivandi', Rachael Dangarembizi ${ }^{1,3}$, Kennedy Erlwanger ${ }^{1}$ 'School of Physiology, Faculty of Health Sciences, University of the Witwatersrand, ${ }^{2}$ Gastrointestinal tract and nutritionM, ${ }^{3}$ Faculty of Medicine, National University of Science and Technology, Box AC 939, Ascot, Bulawayo, Zimbabwe
Nutritional experiences during the perinatal period are known to influence health outcomes later in life. This dietary programming of metabolism and the consumption of fructose-rich diets have been implicated in the increase of metabolic disorders worldwide. Phytochemicals such as ursolic acid (UA), which affect metabolism, may promote metabolic programming in neonates thereby imparting positive health benefits in adulthood. The effects of early administration of UA on subsequent development of complications associated with diet-induced metabolic dysfunction in Sprague Dawley rats were investigated. Six-day old suckling male and female Sprague Dawley rats $(\mathrm{N}=107$ ) randomly received $10 \mathrm{ml} / \mathrm{kg}$ of either $0.5 \%$ dimethylsulphoxide (control), UA, 50\% fructose solution or a mixture of $50 \%$ fructose and UA orogastrically for 14 days. They were then weaned onto normal rat chow and plain drinking water on day 21. At adulthood (day 70), half the number of rats in each treatment group received either plain drinking water or $20 \%$ fructose solution in drinking water for eight weeks. Body mass gain, fasting blood triglyceride concentrations, adiposity and hepatic lipid accumulation were assessed. Fructose consumption in adulthood caused increased $(\mathrm{P}<0.05)$ body mass and circulating blood triglycerides in males whilst it had no effect on adiposity in both sexes. In females, fructose consumption both neonatally and in adulthood caused significantly increased hepatic lipid accumulation (P? 0.0001). Neonatal administration of UA prevented fructose-induced hepatic lipid accumulation in both sexes. Although fructose administration had adverse metabolic effects, neonatal intervention with UA was hepatoprotective and showed great potential in hepatic lipid metabolism control.

Key words: Ursolic acid, metabolic programming, metabolic dysfunction

\section{DL-O-10: IMPROVED SLEEP INERTIA IN THE MORNING IN HEALTHY OLDER ADULTS FOLLOWING EXPOSURE TO BRIGHT BLUE- ENRICHED LIGHT THE PREVIOUS EVENING.}

Karine Schevermaier ${ }^{1}$, Mirjam Münch ${ }^{2}$, Edward J Silva ${ }^{3}$, Joseph M Ronda ${ }^{4}$, Jeanne F Duffy ${ }^{4}$

'School of Physiology, Brain Function Research Group, University of the Witwatersrand, ${ }^{2}$ Sleep Research and Clinical Chronobiology, Institute of Physiology, Charité University Medicine Berlin, Germany, ${ }^{3}$ Department of Preventive Medicine, Brigham and Women's Hospital, Boston, MA, United States, ${ }^{4}$ Division of Sleep Medicine, Harvard Medical School, Division of Sleep Medicine, Brigham and Women's Hospital, Boston, MA, United States

Exposure to light can have acute alerting and circadian phase-shifting effects. This study investigated the effects of evening exposure to blue-enriched (BEL) vs. polychromatic white light (WL) on sleep inertia dissipation the following morning in older adults. Ten healthy older adults (average age $=63.3 \mathrm{yrs}$; 6F) participated in a 13-day study consisting of three baseline days, four days with 2-h 
evening light exposures, wedged between two constant postures for pre- and post- light exposure circadian phase assessment, and 3 final days. Participants were randomized to either BEL or WL of the same irradiance. At 2, 12, 22 and 32 minutes after each wake time during the study, the participants completed a 90-s digit-symbol substitution test (DSST) to assess cognitive throughput. Number of correct DSST responses in the 90s trial was used as the outcome variable. Correct DSST responses increased with time awake $(p<0.0001)$ and across study days in both groups $(p<0.0001)$. There was no main effect of group, although we observed a significant day $x$ group interaction $(p=0.0004)$, whereby participants exposed to BEL performed significantly better on the first two mornings after light exposures than participants in WL (post-hoc $p<0.05)$. These effects were not due to changes in circadian phase or phase angle of entrainment, because both groups showed similar phase-delay shifts. Exposure to blue-enriched white light in the evening significantly improved DSST performance after the following wake time when compared to ordinary white light.R01-AG06072, T32HL07901, F32-AG03169, SAMRC.

Key words: Cognition, light exposure, aging

\section{DL-O-11: POST-EXERCISE EFFECTS ON AORTIC WAVE REFLECTION DERIVED FROM WAVE SEPARATION ANALYSIS IN YOUNG-TO MIDDLE-AGED PRE- HYPERTENSIVES AND HYPERTENSIVES.}

Aletta Millen, Angela J Woodiwiss, Gavin R Norton School of Physiology, Cardiovascular Pathophysiology and Genomics Research Unit, University of the Witwatersrand

Decreases in brachial blood pressure (BP) may occur for several hours following a bout of exercise. Although aortic backward waves predict cardiovascular damage independent of brachial BP, whether decreases in aortic backward waves also occur post-exercise; the extent to which these changes exceed brachial BP changes; and the best method of identifying these changes is uncertain. We examined aortic function at baseline and 15 minutes post-exercise in 20 pre-hypertensive or hypertensive men and women (age: $45 \pm 7$ years). Central aortic pressure, forward (Pf) and backward (Pb) wave pressures, the reflection index (RI) and augmentation pressure (AP) and index (AIX) were determined using applanation tonometry, and wave separation analysis with SphygmoCor software. Decreases in central aortic $(p<0.001)$ but not brachial systolic BP and pulse pressure (PP) occurred post-exercise. In addition, decreases in post-exercise (baseline versus post-exercise) $\mathrm{Pb}(19 \pm 4$ vs $13 \pm 3 \mathrm{~mm} \mathrm{Hg} p<0.0001)$, RI $(72.9 \pm 22.1$ vs $47.6 \pm 12.8 \%, p<0.0001)$, Alx $(26.3 \pm 10.8$ vs $7.8 \pm 11.6 \%, p<0.0001)$ and AP $(9.9 \pm 3.9$ vs $2.8 \pm 3.9 \mathrm{~mm} \mathrm{Hg}$, $p<0.0001$ ), but not Pf were noted. However, decreases in Alx were not correlated with decreases in $\mathrm{Pb}$, and whilst decreases in aortic PP correlated with decreases in $\mathrm{Pb}$ $(p<0.0001)$, no correlations were noted with decreases in
AP or Alx. In pre-hypertensive and hypertensive individuals, aortic backward waves decrease post-exercise; this change is not reflected in brachial BP measurements and is poorly indexed by measures of pressure augmentation.

Key words: Aerobic exercise training, reflected wave, forward wave

\section{DL-O-12: CARDIORESPIRATORY FITNESS LEVELS AND ASSOCIATIONS WITH PHYSICAL ACTIVITY AND BODY COMPOSITION IN YOUNG SOUTH AFRICAN ADULTS IN SOWETO}

Alessandra Prioreschi', Soren Brage ${ }^{2}$, Kate Westgate $^{2}$, Lisa Micklesfield'

'School of Clinical Medicine, Department of Paediatrics, Developmental Pathways for Health Research Unit, University of the Witwatersrand, ${ }^{2}$ MRC Epidemiology Unit, Cambridge

This study aims to describe fitness levels in 423 young black South African adults from Soweto, examining associations with physical activity and BMI. A sub-maximal ramped step test was used to obtain an estimate of maximal oxygen uptake $\left(\mathrm{VO}_{2 \max }\right)$. Physical activity was measured using ActiGraph (GT1M) for one week. Time spent in sedentary $(<100$ counts per minute (cpm)), moderate ( $\geq 2020$ and $\leq 5998 \mathrm{cpm}$ ) and vigorous ( $\geq 5999 \mathrm{cpm}$ ) intensity activity was calculated, and $90 \%$ of participants were considered active according to CDC recommendations. Overweight and obesity was more prevalent in females than males $(35 \%$ vs $8 \%, p<0.001)$. Males had a higher $\mathrm{VO}_{2 \max }$ than females (43.0(4.5) vs 34.5(4.0) $\left.\mathrm{mlO}_{2} / \mathrm{min} / \mathrm{kg}, \quad p<0.001\right)$; spent more time in moderate to vigorous physical activity (MVPA) (83(60-1 11 ) vs 43(25-54)min/day, $p<0.001)$, and less time in sedentary behaviours $(p<0.00)$. Sedentary time $(9$ hours 20 minutes) was not associated with $\mathrm{VO}_{2 \max ^{\prime}}$, however BMI was inversely-, and MVPA positively, -associated with $\mathrm{VO}_{2 \max }$ (both $\mathrm{p}<0.001$ ). Most young South African adults in this study were sufficiently active. MVPA and BMl are both strongly associated with fitness. Males were fitter, more physically active, and had lower BMls than females. The high level of sedentary behaviour in this population is of concern.

Key words: Cardiorespiratory fitness, physical activity, body composition, South Africa, young adults

\section{DL-O-13: EFFECT OF ORALLY ADMINISTERED S-ALLYL CYSTEINE (SAC) ON HEPATIC LIPID ACCUMULATION AND MARKERS OF GENERAL HEALTH IN SUCKLING WISTAR RAT PUPS FED A HIGH- FRUCTOSE DIET}

Busisani Lembede, Jeannette Joubert, Kennedy H. Erlwanger, Eliton Chivandi

School of Physiology, University of the Witwatersrand

There is an increase in childhood obesity and associated complication including non-alcoholic fatty liver disease 
(NAFLD). The beneficial antiobesity properties of garlic and S-allyl cysteine (SAC), a phytochemical derived from garlic, have been investigated in drug-induced adult models of obesity but not in diet-induced models in growing animals. Thus this study interrogated the effects of SAC in high-fructose diet fed rat pups. Sixty-four 6-day old male $(n=32)$ and female $(n=32)$ Wistar rat pups, randomly allocated to four groups, received the following treatment daily for 15 days (postnatal day 6 to 20): I - $10 \mathrm{ml} / \mathrm{kg}$ distilled water (DH), II - 20\% fructose solution (FS), III - $150 \mathrm{mg} / \mathrm{kg}$ SAC, IV - $150 \mathrm{mg} / \mathrm{kg} \mathrm{SAC}+20 \%$ fructose solution (SAC + FS). The pups' blood cholesterol, glucose and triglyceride concentration were then determined. Immediately thereafter the pups were euthanized and blood collected for the determination of surrogate markers of health. Liver samples were collected for glycogen and lipid content determination. The administration of FS or SAC + FS had no effects on the measured parameters across treatment groups. However, in female pups the administration of SAC significantly increased $(P \leq 0.05)$ hepatic lipid content and significantly decreased $(P \leq 0.05)$ hepatic glycogen content. These findings suggest that administration SAC induced lipid accumulation in female rat pups which could predispose them to NAFLD.

Key words: S-allyl-cysteine, hepatic lipid, high-fructose diet

\section{DL-O-14: THE PREVALENCE OF THE METABOLIC SYNDROME AND RELATED DISORDERS IN SOUTH AFRICANS WITH PSORIASIS}

Nasrin Goolam Mahyooden', Nasrin Goolam Mahyoodeen", Nigel Crowther ${ }^{2}$, Mercedes Morrison ${ }^{3}$, Lushen Pillay ${ }^{3}$, Tracy Snyman ${ }^{2}$, Mohammed Tikly ${ }^{3}$

'School of Clinical Medicine, Department of Medicine, University of the Witwatersrand, ${ }^{2}$ National Health Laboratory Service, ${ }^{3}$ University of the Witwatersrand

Psoriasis ( $\mathrm{PsO}$ ) is an immune-mediated inflammatory disorder in which cardio-metabolic co-morbidities are increasingly recognised. There are no data from subSaharan Africa on the relationship between PsO and cardiometabolic disease risk factors. The objective of this study was to determine the prevalence of the metabolic syndrome (MS) and related disorders in PsO patients.Adult PsO patients ( $n=95$ ) were recruited from the Dermatology and Rheumatology clinics at hospitals of the Wits Academic Complex. Demographic, anthropometric and cardiometabolic data were recorded. Disease severity of $\mathrm{PsO}$ was assessed using the psoriasis area and severity index (PASI) scoring method. Components of MS were defined according to the Harmonized guidelines. The mean $( \pm S D)$ age and disease duration were 52.6 \pm 14.6 and $17.6 \pm 13.3$ years, respectively. The mean $\mathrm{BMI}$ and waist circumference were $31.9 \pm 8.64 \mathrm{~kg} / \mathrm{m} 2$ and $101.4 \pm 16.9 \mathrm{~cm}$, respectively. The prevalence $(\%$ [95\% Cls]) of obesity was
$51.6[41.3,61.8]$, T2D was 31.2 [20.6, 41.7], hypertension was 76.8 [68.2, 85.5], hypercholesterolaemia was 54.2 [42.4, 65.9] and psoriatic arthritis (PsA) was 27.4 [18.2, 36.5]. The prevalence of MS was 58.1 [46.6, 69.6] \%. Multivariate logistic regression analysis showed that Indian ethnicity was associated with a higher risk of MS (odds ratio [95\% Cls]: $6.64[1.28,34.4] ; p=0.02)$, as was a higher PASI score $(4.34$ $[1.00,18.9] ; p=0.05)$. A higher socio-economic status was associated with a lower risk of MS $(0.14[0.02,0.84] ; p=0.03)$. The prevalence of obesity and its complications is high in this population suggesting screening for cardiometabolic diseases should form part of routine care in PsO patients.

Key words: Psoriasis, metabolic, syndrome

\section{DL-O-15: INTERACTION BETWEEN HIGH SODIUM AND LOW POTASSIUM INTAKE ON BLOOD PRESSURE IN ADULT SPRAGUE- DAWLEY RATS}

\author{
Lebogang Mokotedi, Aletta Millen \\ School of Physiology, Cardiovascular Pathophysiology and \\ Genomics Research Unit, University of the Witwatersrand
}

While an increase in sodium intake ( $\mathrm{Na}+)$ increases blood pressure (BP), it is uncertain to what extent potassium $(\mathrm{K}+)$ affects $\mathrm{Na}$-induced increases in BP and the mechanism responsible for the increased BP. The aim of the present study was to determine whether $\mathrm{Na}+$-induced increases in BP and changes in vascular reactivity are altered by reductions in $\mathrm{K}+$ intake. Four-month-old male SpragueDawley (SD) rats were randomly assigned to three dietary intervention groups for six weeks: a normal $\mathrm{Na}+(0.3 \%)$, normal $\mathrm{K}+(1.6 \%)$ group (CON, $\mathrm{n}=12)$, a high $\mathrm{Na}+(6 \%)$, normal $\mathrm{K}+(1.6 \%)$ group (NormK+, $\mathrm{n}=12)$ and a high $\mathrm{Na}+$ (6\%), low $K+(0.01 \%)$ group (LowK+, $n=12)$. Tail-cuff BP was measured weekly. At termination, urinary $\mathrm{Na}+$ and $\mathrm{K}+$ concentrations were measured. Vascular reactivity of the mesenteric arteries was also assessed using a wiremyograph. The urinary $\mathrm{Na}+/ \mathrm{K}+$ ratio was higher in the LowK+ compared to CON and NormK+ $(P<0.001)$. The increase in systolic BP over 6 weeks was greater in LowK+ compared to $\mathrm{CON}(\mathrm{P}=0.04)$. The increase in diastolic BP $(\mathrm{mm} \mathrm{Hg})$ was greater in NormK+ and LowK+ compared to CON ( $P=0.05$ and $P=0.02$, respectively). The increase in $B P$ was not different between NormK+ and LowK+ $(P>0.05)$. In mesenteric arteries at termination, the phenylephrine dose-response curves were shifted to the left in NormK+ compared to $\mathrm{CON}(\mathrm{P}=0.02)$ and similar between NormK+ and LowK+ $(\mathrm{P}=0.82) . \mathrm{Na}+$ induced greater phenylephrineinduced contractions which may be responsible for the increase in BP. However, a deficit in $\mathrm{K}+$ intake does not worsen the high $\mathrm{Na}$ + intake effects.

Key words: Sodium, potassium, blood, pressure 


\section{DL-O-16: EXPOSURE TO SECONDHAND SMOKE AMONG PREGNANT WOMEN IN SOWETO, SOUTH AFRICA}

Joanne Pottow, Eckhart Butchmann

School of Clinical Medicine, Department of Obstetrics and Gynaecology, University of the Witwatersrand

Tobacco secondhand smoke (SHS) has long being known for all its negative health effects. This work aimed to determine the SHS exposure rate in the pregnant population of Soweto. This was a prospective, cross sectional study undertaken at Chris Hani Baragwanath Academic Hospital. Soweto serves in excess of two million people, with more than 23000 delivers annually in the hospital. This study used a questionnaire to survey a sample of pregnant women who were post caesarean section. A total of 100 women were interviewed. Twenty one percent reported to be exposed to SHS at home and $18 \%$ of the employed participants exposed at work. Forty three percent of the participants lived with a regular smoker and $73 \%$ had banned smoking in their house. However, even though the bans had been put in place, smoking still occurred in some of their homes. There was a statistically significant difference in the number of regular smokers that the participant lived with, with SHS-exposed participants being more likely to live with a regular smoker than with no regular smokers in the house. Ninety one percent of participants were aware that SHS could have a negative effect on their babies while pregnant, and knew about health risks with SHS. This study showed that in spite of strict anti-tobacco laws, a high percentage of pregnant women reported to be exposed to SHS at home and at work. Most were aware of the health risks of SHS, and tried to ban smoking in their homes.

Key words: Secondhand smoke in pregnancy

\section{DL-O-17: TIME TO THE PEAK OF THE AORTIC FORWARD WAVE DETERMINES THE IMPACT OF AORTIC BACKWARD WAVE AND PULSE PRESSURE ON LEFT VENTRICULAR MASS.}

Grace Tade, Gavin R Norton, Hendrick L. Booysen, Moekanyi J. Sibiya, Imraan Ballim, Elena Libhaber, Olebogeng H.I. Majane, Angela J. Woodiwiss

School of Physiology, Cardiovascular Pathophysiology and Genomics Research Unit, Faculty of Health Sciences, University of the Witwatersrand

There is extensive evidence that in hypertension, aortic blood pressure (BP) adds to brachial BP when risk predicting. This effect is largely accounted for by aortic backward waves. The factors which determine the adverse impact of backward waves on end-organs are uncertain. We aimed to determine the degree to which an extended time to the peak of the aortic forward wave (Ft) or early wave reflection time (Rt) enhance associations between backward wave pressures (Pb) and left ventricular mass index (LVMI). In 812 adult participants from a community sample we assessed aortic haemodynamics and LVMI (echocardiography). An interaction between $\mathrm{Ft}$ and $\mathrm{Pb}$ was independently associated with LVMI ( $p \leq 0.0001$ ) and LV hypertrophy (LVH) ( $p=0.001)$. The Ft-Pb interaction translated into an increase in the independent association between $\mathrm{Pb}$ and LVMI ( $\mathrm{p} \leq 0.0001$ for comparison of slopes and strength of relations) or $\mathrm{LVH}$ ( $\mathrm{p} \leq 0.01$ for comparisons of Odds ratios). A markedly better ability of $\mathrm{Pb}$, but not forward wave pressures to detect LVH was noted in the highest as compared to the first three quartiles of Ft $(p \leq 0.01)$. In contrast, Rt failed to influence the impact of $\mathrm{Pb}$ on LVMI. In conclusion, time to the peak of the aortic forward wave, but not early wave reflection markedly influences the impact of aortic backward wave pressure and hence aortic PP on LVMI and LVH in adults. This data suggest that underlying the time to the peak of the forward wave may be an important risk factor in African ancestry.

Key words: Central blood pressure, reflected waves, left ventricular mass index.

\section{DL-P-1: BREAST AND CERVICAL CANCER AWARENESS AND PRACTICE AMONG WOMEN IN INFORMAL SECTOR, NIGERIA}

Adenike Esther Idowu 1,2, Emmanuel Amoo 1'3

'Department of Demography, School of Public Health, Faculty of Health Sciences, University of the Witwatersrand, '2Department of Sociology, College of Business and Social Sciences, Covenant University, Ota, Ogun State Nigeria, ${ }^{3}$ Department of Demography and Social Statistic, College of Business and Social Sciences, Covenant University, Ota, Ogun State Nigeria.

There is no general consensus on why the life time risk of dying from cancer in African women is several times higher than that of women from developed countries. However, due to little or no awareness and the low level of cancer screening in African women the window of opportunity for prevention and cure is often wasted. This study examined the relationship between breast and cervical cancer awareness and screening among women in informal sector to be able to develop a contextual program for timely intervention. We performed a crosssectional study in women between the ages of 15 and 49 years to examine the correlation between cancer awareness and participation in cancer screening. This work adopted both quantitative and qualitative research approaches. Outcome measures included awareness of breast and cervical cancer, how it can be prevented or detected, and the availability and actual participation in screening exercises. The results from the study show that while women are familiar with breast cancer, little is known about cervical cancer and the awareness of the former is not correlated with participation in cancer screening. The lack of enthusiasm to seek cancer screening is rooted in three key factors: lack of specific information, religious 
believes economic and inadequate screening facilities. The study thus recommends that, policies and programs aimed at breast and cervical cancers awareness and early intervention should address these underlying problems of accessibility of screening facilities, cost of service and appropriate way of enlightening the informal sector women on the benefit of cancer screening.

Key words: Women, Breast, Cervical cancer, Awareness, Practice

\section{DL-P-2: MEN'S SEXUAL HEALTH PROBLEMS ANDAPPRAISAL OF WIVES COPING STRATEGIES}

Emmanuel O. Amoo ${ }^{1,2}$, Adekunbi K. Omideyi ${ }^{3}$, Theophilus O. Fadayomi ${ }^{4}$, Mofoluwake P. Ajayi ${ }^{5}$, Adenike E. Idowu' \& Gbolahan A. Oni ${ }^{6}$

'Andrew Mellon Foundation Postdoctoral Fellow, Demography and Population Studies, Schools of Public Health \& Social Sciences, University of Witwatersrand, Johannesburg, South Africa, ${ }^{2}$ Demography and Social Statistics, School of Social Sciences, Covenant University, Ota, Nigeria, ${ }^{3}$ Department of Demography and Social Statistics, Obafemi Awolowo University, Ile Ife, Nigeria, ${ }^{4}$ Economics Department, Elizade University, llaramokin, Ekiti State, Nigeria, ${ }^{5}$ Sociology Department, School of Social Sciences, Covenant University, Ota, Ogun State, Nigeria, ${ }^{6}$ Demography and Social Statistics, School of Social Sciences, Covenant University, Ota, Ogun State, Nigeria

Systematic studies on the relationship between men's sexual dysfunction and marital conflict are emerging. However, the coping strategies adopted by wives under such circumstances are lacking in the literature. Male sexual functioning is vital to marital success and its limitation impairs marital relationship, causes intolerable cohabitation, and can stimulate infertility, and stigma in sub-Saharan Africa. The coping strategies employed by women to these encounters have the potential to lessen or prevent maladaptive outcomes and engender a sustainable intact (stable) marital relationship. Thus study therefore examined the coping strategies adopted by women whose husbands have reproductive health challenges in two of the five states with highest proportion of divorced/ separated in Nigeria. Four focus group discussions were organized in two local government areas from the two states. The women were recruited from a quantitative couple-study where the men have experienced any sexual health problems. The responses were transcribed and analyzed using a 'systematic-content-analysis' technique with simple thematic organisation of summaries and systematic typologies of participants' responses. The results revealed coping strategies employed by the women as: seeking guidance from their religious leaders and family doctors, physical-sexual-therapy, abstinence/ religiosity and concubinage. The participants indicated it is sacrilege reporting husbands' sexual health problems publicly. The study concludes that husband's sexual ability is crucial to sustenance of the marital relationship: that religious leaders and family doctors are indispensable mediators in husband-wife conflict management but concubinage could make wives vulnerable to STIS/HIV and AIDS. The authors recommend public enlightenment on men's sexual health problems for the wives while medical officers (family doctors) and religious leaders could be trained on family-conflict management as a strategy to stem increasing marital conflict/dissolution in the study locations and in sub-Saharan Africa.

Key words: Wives, coping strategy, men sexual health problem, conjugal relationship

\section{DL-P-3: CHRONIC KIDNEY DISEASE EPIDEMIOLOGY COLLABORATION- DERIVED GLOMERULAR FILTRATION RATE PERFORMS BETTER AT DETECTING PRE- CLINICAL END ORGAN CHANGES THAN ALTERNATIVE EQUATIONS IN BLACK AFRICANS.}

Hendrik Booysen, Angela Woodiwiss, Andrew Raymond, Pinhas Sareli, Hon-Chun Hsu, Patrick Dessein, Gavin Norton

School of Physiology, Cardiovascular Pathophysiology and Genomics Research Unit, Faculty of Health Sciences, University of the Witwatersrand

Because the relationship between creatinine and glomerular filtration rate (GFR) varies between ethnicities, neither of the more recently developed equations (MDRD, nor the Chronic Kidney Disease Epidemiology Collaboration CKD-EPI) for estimating GFR (eGFR) perform as well in African as they do in Caucasian populations. However, there are no studies in black African populations that have compared the various equations for eGFR in associations with end-organ changes. In 1221 randomly recruited participants of black African ancestry in South Africa, we evaluated serum creatinine concentrations, echocardiographic left ventricular mass index (LVMI) ( $n=833)$, carotid-femoral (aortic) pulse wave velocity (PWV) ( $n=1053)$ and carotid intima-media thickness (IMT) $(n=633)$. We calculated eGFR from the Jelliffe, 5 CockcroftGault, Salazar-Corcoran, MDRD and CKD-EPI equations. After multivariate adjustments, eGFR calculated from all formulae was inversely associated with LVMI $(p<0.0001)$ and PWV ( $p<0.05$ to $<0.001)$, but not with IMT ( $p>0.07)$. However, while eGFR determined from all equations except Cockcroft-Gault lean body weight or adjusted body weight was independently associated with LV hypertrophy (LVH) (n=390 of 833); CKD-EPI-derived eGFR, but not eGFR determined from alternative equations was independently associated with an increased PWV ( $n=88$ of 1053). eGFR derived from the CKD-EPI and MDRD equations showed a better performance (area under the receiver operator characteristic curve) for the detection of LVH $(p<0.0005)$ than eGFR determined from alternative equations. In conclusion, in black Africans, eGFR derived 
from the CKD-EPI equation is better at detecting endorgan measures than eGFR derived from either the MDRD or alternative equations.

Key words: Derived glomerular filtration rate, end organ changes

\section{DL-P-4: AN AUDIT OF PAEDIATRIC PATIENTS PRESENTING FOR DENTAL GENERAL ANAESTHETIC AT WITS IN 2011}

Joy Shackleton, Natalie Gray

School of Oral Health Sciences, Oral Rehabilitation, University of the Witwatersrand

Early childhood caries (ECC) is preventable, but widespread. This study described the patients accessing the Dental General Anaesthetic (DGA) service. Further aims were to establish the treatment provided, and the number of unscheduled patients. This was a retrospective study of a sample of the 516 patients treated under DGA at Wits in 2011 . The patients were 16 years or younger, and for 299 of them data was retrieved from the patient files, the theatre register and/or the daysheet, captured in Excel and exported into SPSS Version 21 for analysis. The average age of healthy children was 4.9 years. Mentally or physically compromised children were $13.7 \%$ of the group with an average age of 9.4 years. Most children were from low socioeconomic groups and $55 \%$ of patients travelled more than $10 \mathrm{~km}$ for treatment. The majority $(79.6 \%)$ were self-referred to Wits Dental Hospital (WDH). Of the remaining $20 \%$, healthy children were referred more often by private practitioners, and compromised children were referred from other hospitals. The average waiting time for treatment was 5 months, and treatment was almost exclusively extractions. The mean number of extractions was 8.88 per child. $17.4 \%$ of patients attended without appointments, but received treatment despite this. Patients accessing DGA at WDH in 2011 were pre-schoolers from a low socioeconomic background, who presented with advanced early childhood caries. This necessitated multiple extractions under DGA. Caries prevention and early detection would reduce the financial and biological costs of treatment.

Key words: Early childhood caries, dental general anaesthetic, paediatric dentistry

\section{DL-P-5: DEVELOPMENT OF A METHOD FOR STUDYING THE IN VITRO FORMATION OF FOAM CELLS FROM HUMAN MONOCYTES}

Maile George Ralefatane', Eleanor Cave ${ }^{2}$, Nigel Crowther $^{2,3}$ 'School of Pathology, Department of Chemical Pathology, 2University of Witwatersrand, ${ }^{3} \mathrm{NHLS}$

Atherosclerosis is a chronic inflammatory disease characterized by cholesterol accumulation within the walls of arteries. Formation of macrophage foam cells (FC) is a major hallmark of early stage atherosclerotic lesions. Currently there is a lack of suitable cellular models to study FC formation in vitro. Therefore, the aim of this study was to develop a method for converting peripheral blood mononuclear cells (PBMC) into FCs. Blood was collected in EDTA tubes. Buffy coats were harvested by centrifugation, separated by Ficoll-Hypaque gradient (density $1.07 \mathrm{~g} / \mathrm{ml}$ ) and the PBMC layer isolated. The monocytes were washed three times in sterile PBS and cultured overnight with RPMI medium. After 24 hours of culture the monocytes adhered to the culture plate and non-adherent lymphocytes were removed through washing with RPMI. The monocytes were then differentiated into macrophages by incubation over 5 days in standard media supplemented with macrophage colony-stimulating factor (M-CSF $(100 \mathrm{ng} / \mathrm{ml}))$. FC formation was induced through exposure of macrophages to OxLDL at a concentration of 3.0 Â $\mu \mathrm{g} /$ $\mathrm{ml}$ for 48 hours. FC formation was determined by Oil red $O$ staining of the intra-cellular lipid. The addition of OxLDL to macrophages resulted in enhanced intracellular lipid accumulation and under microscopic examination they were seen to exhibit classic FC morphology with pronounced staining of intra-cellular lipid droplets. This work resulted in the development of a method allowing for the in vitro generation of FCs from human monocytes and represents a useful tool for studying the molecular mechanisms involved in the control of FC formation.

Key words: Methodology, Monocytes, Foam, Cells

\section{DL-P-6: THE ASSOCIATION BETWEEN VIOLENCE AND MENTAL HEALTH IN ADOLESCENT GIRLS: RURAL-URBAN DIFFERENCES}

Mercy Manyema, Shane Norris, Linda Richter

School of Public Health, Developmental Pathways for Health Research Unit, Faculty of Health Sciences, University of the Witwatersrand

Poor mental health accounts for a large proportion of the disease burden in young people in all societies and it is strongly related to other health and developmental concerns in young people. Exposure to community and personal violence may increase the risk of poor mental health. The aim of this study is to compare the differences in the association between violence and mental health in adolescent girls from rural and urban communities. Data from the Birth to Twenty Plus and Ntshembo studies was used to estimate the prevalence of psychological distress and violence in urban and rural communities respectively. Using regression models, we determined the association between mental health and personal violence, adjusting for other individual, household and community characteristics. $32 \%$ of the urban girls and $17 \%$ of the rural girls showed psychological distress and $23 \%$ vs $14 \%$ 
reported experiencing personal violence respectively. The unadjusted odds of having psychological distress was approximately 1.8 times higher in girls who experienced personal violence compared to those who didn't in both communities. Adjusting for household stressful events and socio-economic status reduced the odds to OR $1.6195 \%$ $\mathrm{Cl}$ 1.01-2.67; $\mathrm{p}$-value -<0.05) in the urban adolescents and to 1.3 (0.63-2.79; $p$-value- $>0.05$ ) in the rural girls, indicating the possible mediating role of the household factors. Both rural and urban girls who experience personal violence are at risk of experiencing psychological distress and household factors seem to play a significant role in this association.

Key words: Violence, mental health, adolescents, rural-urban

\section{DL-P-7: WHICH INDEXES OF AORTIC FUNCTION BEST ADD TO BRACHIAL PULSE PRESSURE ASSOCIATIONS WITH END- ORGAN CHANGES INDEPENDENT OF PULSE WAVE VELOCITY IN A GROUP OF AFRICAN ANCESTRY?}

\author{
Moekanyi J Sibiya, Angela J Woodiwiss, Hendrik L Booysen, \\ Grace Tade, Carlos D Libhaber, Imraan Ballim, Pinhas Sareli, \\ Gavin R Norton.
}

School of Physiology and Clinical Medicine, Cardiovascular Pathophysiology and Genomics Research Unit, Faculty of Health Sciences, University of the Witwatersrand, Johannesburg, South Africa

There is increasing evidence that several indices of aortic function add to cardiovascular risk prediction. However, those indices that add to rather than replace brachial pulse pressure (PP) associations with cardiovascular endorgan measures independent of aortic pulse wave velocity (PWV) are uncertain. In 1197 community participants of African ancestry we assessed aortic function using radial applanation tonometry and end-organ changes from echocardiography (left ventricular mass index [LVMI]) ( $n=812)$, carotid ultrasound (intima-media thickness [n=622]) and estimated glomerular filtration rate $(n=1178)$. Central aortic PP (PPC), 1/aortic-to-brachial PP amplification (PPamp), aortic backward wave pressure $(\mathrm{Pb})$, and aortic reflection magnitude ( $\mathrm{RM}=\mathrm{Pb} /$ forward wave pressure), but neither aortic augmented pressures $(\mathrm{Pa})$, nor index (Alx) were associated with all end-organ measures independent of brachial PP. In multivariate models with the inclusion of PPc or Pb and brachial PP; $\mathrm{PPc}$ or $\mathrm{Pb}$ replaced brachial PP in relations with end-organ measures or the presence of LV hypertrophy or chronic kidney disease, but added little to the models. In contrast, with the inclusion of brachial PP and 1/PPamp or RM in multivariate models, brachial PP-end-organ relations were retained, whilst 1/PPamp $(p<0.0005)$ and $R M(p<0.01$ to $<0.0001$ ) further added to the models. Relations between 1/PPamp or RM and end-organ changes remained unchanged with adjustments for PWV. In conclusion, 1/ PP amplification and the reflected (backward) wave magnitude (RM), but not PPC, Pb, $\mathrm{Pa}$, or Alx add to brachial PP associations with end-organ measures and these effects are independent of aortic PWV.

Key words: Pulse, Pressure, Amplification

\section{DL-P-8: A RETROSPECTIVE STUDY INVESTIGATING THE EFFICACY OF A 12 WEEK EXERCISE PROGRAMME IN MEN AND WOMEN WITH LOW BACK PAIN IN GAUTENG}

Natalia Neophytou ${ }^{1,2}$, Chantelle Etsebeth', Estelle Watson'

Centre for Exercise Science and Sports Medicine, School of Therapeutic Sciences, Faculty of Health Sciences, University of the Witwatersrand, Johannesburg, South Africa

Low back pain (LBP) is a world-wide occurring health problem. It is said to be related to a loss in quality of life as well a disruption in activities of daily living. In developed countries, approximately $70 \%$ of the population suffer from LBP. Among the healthy population alone in South Africa, it is estimated that $35,8 \%$ of the population experience LBP. To assess whether an exercise programme, that is participated in for 12 weeks, will decrease the amount of pain experienced as well as improve postural and pelvic stability in those who suffer from low back pain. A retrospective analysis was employed to investigate the effects a 12 week LBP exercise programme had in 10 participants (age: 59,2 $\pm 8,26$ years). Pain was assessed using an Oswestry pain questionnaire, postural stability was assessed using a Biodex Balance SD system and pelvic stability was assessed using a step-down and Trendelenburg test. The intervention included 12 weeks of biweekly exercise which occurred in 60 minute bouts. Exercises included retraining of the core stabiliser muscles as well as general core muscle strengthening and general conditioning. Exercises were modified/adapted for each individual to ensure optimal exercise benefit. None of the subjects showed improvements in all variables, yet, an improvement in three of the variables were found in $40 \%$ of subjects, an improvement in two variables was found in $30 \%$ of subjects and an improvement in a single variable was found in $30 \%$ of subjects. Overall most participants improved in reducing the amount of pain experienced, followed by an improvement pelvic stability. However there was a decline in postural stability in all except for 2 participants, yet this was not statistically significant. It can therefore be stated that the exercise intervention had a beneficial effect on all the subjects that participated in the study. Exercise as an intervention is cost effective and has the potential to specifically improve the pain experienced with low back pain. Due to uniqueness of all people, some individuals may respond better to exercise than others.

Key words: Exercise programme, low back pain 


\section{DL-P-9: MULTICOLOUR FLOW CYTOMETRY FOR STUDYING IMMUNE PARAMETERS THAT DIFFERENTIATE DIFFERENT PHASES OF ACUTE PANCREATITIS}

Pascaline Fonteh, Martin Smith, Martin Brand

School of Clinical Medicine, Department of Surgery, Faculty of Health Sciences, University of the Witwatersrand

Multicolour flow cytometry is a powerful means of characterizing immune cells at the single cell level in one experiment. By using this technique multiple epitopes on a single cell can be detected to phenotype immune cells at a fine level e.g., memory versus naïve phenotype. Multicolour flow cytometry has been used extensively in HIV/AIDS studies over the last decade to characterize changes in immune cells during the course of disease, in treatment with HAART and to identify $T$ cell subsets predictive of progression to AIDS. These studies and others highlight the diverse scientific questions that have been addressed in HIV immunology using flow cytometry.To date no study has employed multicolour flow cytometry to assess immune cells in acute pancreatitis (AP), an inflammatory disease of the pancreas commonly caused by alcohol abuse. Given the immense data generated by flow cytometry experiments, such a study will provide insights into the complexity of the immune responses taking place during the course of AP. This data would be correlated with clinical outcomes of patients, and potentially open avenues for immunomodulation research in AP. The findings will also provide information on the management of AP, mitigating the often fatal outcome from associated complications. Preliminary findings looking at cytokine production between AP patients of different severities showed that the systemic response is polarized towards a Th17 rather than a Th1 cytokine response, requiring further exploration.

Key words: Acute pancreatitis, flow cytometry, stages, prognosis
DL-P-10: LEFT VENTRICULAR DIASTOLIC DYSFUNCTION IS ASSOCIATED WITH AORTIC BACKWARD WAVE PRESSURE, BUT NOT STIFFNESS IN A PREDOMINANTLY YOUNG-TO-MIDDLE AGED COMMUNITY SAMPLE.

Vernice Peterson, Angela Woodiwiss, Carlos Libhaber, Andrew Raymond, Pinhas Sareli, Gavin Norton

School of Physiology, Cardiovascular Pathophysiology and Genomics Research Unit, University of the Witwatersrand

There are limited proven therapies for heart failure with a preserved ejection fraction hence identifying the mechanisms responsible is of importance. Central to the pathogenesis are increases in aortic stiffness and enhanced aortic backward waves which produce diastolic dysfunction (DD). Whether the impact of backward wave pressures on left ventricular (LV) DD antedates the effects of aortic stiffness is uncertain. Therefore, we compared the relative contribution of various aortic hemodynamic parameters to preclinical DD in a predominantly youngto-middle aged community-based sample. In 524 randomly selected participants of African ancestry we assessed central aortic pulse pressure (PPC), forward wave pressure (Pf), backward wave pressure (Pb), augmented pressure (Pa) and aortic pulse wave velocity (PWV). LV mass index (LVMI), early to late trans-mitral velocity (E/A) and $E /$ velocity of myocardial tissue lengthening (E/e') were determined using echocardiography. Independent of confounders including LVMl; PPC $(p<0.002), \quad \mathrm{Pb}$ $(p<0.0005), P a(p<0.002)$, and Pf $(p<0.02)$, but not PWV were independently associated with $\mathrm{E} / \mathrm{e}^{\prime}$ (but not with $E / A)$. With adjustments for confounders, PPC $(p<0.005)$, $\mathrm{Pb}(\mathrm{p}<0.002)$ and $\mathrm{Pa}(\mathrm{p}<0.001)$, but not Pf or PWV were independently associated with $E / e^{\prime} \geq 12$ (moderate-tosevere $D D, n=69)$. The independent relations between PPc and $E / e^{\prime}$ or moderate-to-severe DD were not affected by adjustments for PWV, or Pf, but were abolished with adjustments for $\mathrm{Pb}$. In conclusion, in a predominantly young-to-middle aged community sample, the impact of backward wave pressures on LV DD antedates the effects of aortic stiffness.

Key words: Aortic function, left ventricular diastolic function, backward waves 


\section{EDUCATION POLICY AND SYSTEMS}

\section{EPS-O-1: A METHODOLOGY FOR UNDERTAKING SCOPING REVIEWS}

Patricia Mclnerney', Micah Peters², Christina Godfrey³, Cassai Soares ${ }^{4}$, Hanan Khalili, Deborah Parker ${ }^{6}$

'Centre for Health Science Education, Faculty of Health Sciences, University of the Witwatersrand, ${ }^{2}$ Joanna Briggs Institute-Adelaide, ${ }^{3} \mathrm{JBI}$-Queen's University, Canada, ${ }^{4}$ University of Sao Paolo, Brazil, ${ }^{5}$ Monash University, Melbourne, Australia, GUniversity of Queensland, Australia

Scoping reviews are useful to map the key concepts related to an area of research; to clarify definitions or identify the boundaries of a topic. They may be undertaken as a preliminary exercise prior to a systematic review or to identify gaps in the literature. They include a clearly stated title, objective/s and review question. Inclusion criteria should state the types of participants being considered in the review $(\mathrm{P})$; the core concepts being studied $(\mathrm{C})$ and the context (C). A comprehensive search of the literature is undertaken in order to identify both published and unpublished data. Extraction of results is referred to as charting the results. An initial charting table would include the author (s), year of publication, origin/ country of origin, aims/purpose, study population and sample size (if applicable), methodology / methods, intervention type, comparator and details of both, duration of intervention, outcomes and key findings. Charting the data may become iterative and require updating the data. Results may be presented as a map, tables and charts. The results can be grouped into conceptual categories. The diagrammatic presentation of the results must be accompanied by a narrative summary. The discussion must refer back to the objectives of the review. Finally, the review findings can be used to develop implications for research and in some instances for practice.

Key words: Scoping reviews, methodology

\section{EPS-O-2: INFRASTRUCTURE NEEDS AND END-USER SUPPORT FOR REDCAP WITHIN THE FACULTY OF HEALTH SCIENCES AT THE UNIVERSITY OF THE WITWATERSRAND}

Irma Maré, Mapule Nhlapho

School of Clinical Medicine, Department of Surgery/Biomedical Informatics
REDCap (Research Electronic Data Capture) is a free, web-based, and user-friendly electronic data capture (EDC) and management tool for biomedical research and clinical trials. Each organization who uses the software is responsible for hosting its own installation of REDCap, and supporting its end-users. The objective of this study was to measure the infrastructure and end-user support needs of the Faculty, to ensure adequate resource allocation for continued service delivery. REDCap has experience rapid growth and demand in the last 12 months. Wits has over 1400 users in the Faculty of Health Sciences, with around 400 individual logins per day, and around 150 support email interactions a week. Across all projects, there are over 590000 records stored on the Wits REDCap server. Server infrastructure costs about R 100000 over 3 years, and enduser support requires 1.2 - 1.5 FTE's from an experienced postgraduate individual. Further investigation is needed for financial projections over the next 12 months.

Key words: REDCap, clinical informatics, research data management

\section{EPS-O-3: IMPROVING HEALTH SCIENCES EDUCATION: A CONCEPTUAL FRAMEWORK AND MODEL OF FACTORS AFFECTING ICT USAGE}

Ann George', Martie Sanders ${ }^{2}$

'Health sciences education, ${ }^{2}$ APES

Recent decades have seen the pervasive influx of expensive technologies into education. The potential benefits of the introduction of ICT have been limited either by underuse or ineffective uptake. The barriers to effective uptake of ICT have been studied but the results tend to be fragmented making it difficult for practitioners to have a holistic picture of how they could be applied to improve practice. This study presents a holistic literaturebased conceptual framework and a theoretical model for the teachers' response to an ICT strategy. Content analysis of 108 empirical studies conducted between 1992 and 2016 identified 61 factors affecting ICT usage. Because educational practitioners who want to improve their practice might struggle to process, in a meaningful way, the isolated factors identified, these were clustered 
and mapped. Using the map together with the findings from a case study in secondary education, a theoretical model was developed. The clustering in the concept map reduces the factors into categories and sub-categories and shows the hierarchical relationships between the factors, focussing on institutional and educator-related aspects. The theoretical model developed can be used to explain why educators use ICT in particular ways, providing a basis for improving ICT usage by practitioners. Based on the theory of planned behaviour, the model emphasises attitudes and beliefs as important determinants of educator's intentions to use ICT, and their actual usage, but adds the knowledge and skills which underpin practitioners' attitudes and beliefs, suggesting these as focal areas in programmes to improve ICT uptake. ICT can be used to support transformation in health sciences education, but medical educators need training in dealing with new technologies and new pedagogical approaches to promote effective integration.

Key words: ICT integration, educator factors, conceptual framework, health sciences education

\section{EPS-O-4: COBHSE DEFINING COMMUNITY-BASED EDUCATION IN THE FACULTY OF HEALTH SCIENCES}

Abigail Dreyer', Gaolatlhe Mothoagae ${ }^{2}$

'School of Clinical Medicine, Department of Department of Family Medicine, ${ }^{2}$ Centre For Health Science Education

The history of CBE in the Faculty of Health Sciences spans over many years with some departments in the Faculty training students in the communities for more than 30 years. In 2000, a group of individuals interested in education at a community level from various schools and departments came together and formed what is still referred to as the Faculty's Committee for CommunityBased Health Sciences Education (COBHSE). Currently all students in the different academic programmes in the Faculty are exposed to training and education in communities. COBHSE's core function is developing and advising on the Faculty policy for Community Based Education (CBE) at the university.To reach consensus on a working definition to best describe $C B E$ and define the $C B E$ activities in the Faculty of Health Sciences. A request was sent to 23 members of COBHSE, using Socrative.com(). Participants were asked to choose one definition from a list of three definitions commonly used for CBE that would best describe their understanding of $\mathrm{CBE}$. A response rate of $56 \%(N=23: n=13) .69 \%$ of the participants reached consensus on one definition."Community Based Education is about the facilitation of learning in, with, for, and from the community, rendering relevant, meaningful and mutually agreed upon learning outcomes for health professionals and services to the populations in a community setting. CBE promotes active citizenship and social responsibility in learners as it is based on partnerships and reciprocity between communities and the educational institutions. Ultimately, CBE seeks to produce graduates who are available for improving access and enhancing quality health care for all." Extensive literature demonstrates that a number of challenges and misconceptions still exist in implementing CBE. CBE offers the learning opportunity for students to develop an understanding of the social, economic and community-based influences on the health of individuals and groups. Consensus on a definition will assist in developing the elements needed for the ongoing monitoring of CBE activities

Key words: Community-based education, undergraduate teaching, interprofessional education

\section{EPS-0-5: WHERE IS MY FATHER?...........THE ASSOCIATION BETWEEN SINGLE FEMALE HEADEDNESS AND TEENAGE PREGNANCY IN SOUTH AFRICA}

Sibusiso Mkwananzi, Clifford Odimegwu

'School of Social Sciences and Public Health, Department of Demography and Population Studies, ${ }^{2 H O D}$ Demography and Population Studies

The question of teenage pregnancy (TP) remains a global challenge and local studies have emerged identifying some predictors of the phenomenon. However, the association of single female headedness (SFH) has received little attention. This study aims to investigate the association of SFH at household- and communitylevels. A sample of 25,492 female adolescents was obtained from the 2011-2013 General Household Surveys. Analysis comprised descriptive statistics and multilevel binary logistic regression in the MLWiN programme.SFH increased the odds of TP at household level (OR=1.42). Likewise, medium and high community levels of SFH were associated with increased odds of TP [OR=1.11 and 1.23, respectively]. This may be linked to sex composition of SFH households as $88 \%$ possessed males within them and TP was highly associated with sex composition. It is possible that due to household paternal absence a teenage female may be vulnerable to sexual abuse by resident men, thereby increasing her chances of pregnancy. It is necessary for government to support and target such households early to prevent teenage childbearing and related risks. Participatory creative yet educational programmes involving single mothers and their children from an early age could possibly benefit both mother and child.

Key words: Teenage pregnancy, single female headed households, multilevel modelling, social disorganisation theory 


\section{EPS-O-6: CARE AND RESOURCE UTILISATION OF TYPE 2 DIABETES MELLITUS IN A PRIVATELY MANAGED HEALTH CARE ORGANISATION IN SOUTH AFRICA}

Lovina Naidoo', Neil Butkow', Paula Barnard², Jan Bezuidenhout ${ }^{3}$, Elena Libhaber ${ }^{4}$

Faculty of Health Sciences, Department of Pharmacy and Pharmacology, ${ }^{2}$ School of Therapeutic Sciences WIT's, ${ }^{3 S}$ anlam Health, Strategic Business Development, ${ }^{4}$ School of Clinical Medicine, Faculty of Health Sciences WIT's

The costs of type 2 diabetes mellitus (T2DM) care and its complications (mainly cardiovascular, retinopathy, neuropathy) are increasing, both for the diabetic patient and for the health insurer. Optimal management of T2DM was found to improve outcomes and reduce healthcare costs. The aim of this study was to estimate the costs and resource utilisation of T2DM patients ( $n=849$ with mean age $55+/-14$ years) within a South African private managed health care organisation; in a capitated model (CM) $F / M=40 / 60 \%$, and comparing it to a fee for service model (FSM) $F / M=45 / 55 \%$. A retrospective study of claims data of T2DM patients in both models from 2012 was performed and costs per annum calculated in ZAR. The claims were classified using ICD10 codes. Resource utilisation was categorised according to their medicine specific claims; hospital claims; auxiliary services and physician visits claims. In 2012, the total healthcare cost for diabetic patients on the CM ( $n=562$ ) was R30, 548,259 \{Services and visits R11, 735,742; Medicines R5, 517,232; Hospital R8, 050,925; CM annual fee R5, 244,362\} and on the FSM ( $n=287)$ was R13, 549,158 \{Services and visits R5, 832,255; Medicines R3, 245,063; Hospital R4, 471,840\}. Frequency of use of antihypertensive drugs and statin therapy $\{69 \%$; (CM) 68\% (FSM)\} was sub-optimal when measured against the latest guidelines. Hospitalisation, an important contributor to the increase in healthcare costs, appeared to be high in both models. Prevention of T2DM related complications and hospitalisations would reduce healthcare costs and improve patient outcomes.

Key words: T2DM, Costs, Healthcare, Outcomes

\section{EPS-0-7: FACTORS AFFECTING HEALTH CARE SEEKING BEHAVIOUR IN GAUTENG, SOUTH AFRICA, 2013.}

Adams Abaerei, Jabulani Ncayiyana

Department of Epidemiology and Biostatistics, School of Public Health, Faculty of Health Sciences, University of the Witwatersrand

In South Africa, health care access to for all is constitutionally enshrined; yet considerable inequities remain due to various reasons. There are limited studies on health care seeking behaviour especially of vulnerable populations such as immigrants in South Africa. This study aimed to determine health care seeking behaviour and factors affecting it among residents of Gauteng Province, South Africa. We conducted secondary data analysis of data from a Quality of Life survey carried out by Gauteng City-Region Observatory in 2013. Coarsened exact matching was used to improve estimation of causal effects. Then stepwise multiple logistic regression was employed to identify factors associated with health care seeking behaviour. From a total of 27,490 participants interviewed, $95.7 \%$ reported they usually utilised health services while the rest (4.3\%) reported not having sought health care services of any type when needed. Higher odds of reported health care seeking were associated with being female $(O R=2.18,95 \% \mathrm{Cl}: 1.88-2.53 ; p<0.001)$; being white compared to being African $(O R=2.28,95 \%$ $\mathrm{Cl}$ : $1.84-2.74 ; p<0.001$ ) and having medical insurance. In contrast, lower odds of seeking health care were associated with being an immigrant $1 O R=0.61,95 \%$ Cl: $0.53-0.70 ; p<0.001)$. The results indicate that there is a need to improve the quality of public health care services and perception towards them as improved quality increases the choice of health care. There is also a need to incorporate immigrant populations in the design and implementation of health programs and policies.

Key words: Healthcare seeking behaviour, quality of health care, immigrants, traditional healers

\section{EPS-0-8: PROVIDER PERCEPTIONS OF THE QUALITY OF POST-RAPE CARE IN EKURHULENI DISTRICT}

Brenda Sulile Skosana, Lenore Manderson

Department of Social and Behaviour Change Communication, School of Public Health, University of the Witwatersrand

Rape and HIV are major public health issues in South Africa. Rape has negative short and long term physical and psychological health impacts. The national government has clinical guidelines to improve the quality of care for rape survivors; however, the needs of survivors remain largely unmet. The aim of the study was to explore the knowledge, attitudes and experiences of Clinical Forensic Medical Services (CFMS) health care providers from three facilities in Ekurhuleni District in Gauteng, and to assess the quality of post rape care, in accordance with the National Department of Health Rape and Sexual Assault Policy and Clinical Management Guidelines. A qualitative case study approach was used in three CFMS. A total of 17 participants representing different categories of staff were interviewed using a semi-structured interview guide, interviews were audio recorded and transcribed verbatim. Thematic analysis was conducted. Participants demonstrated professionalism and confidence in managing rape survivors, but they were uncertain about the sexual assault policies and clinical management guidelines. As a result practices such as the provision of post-exposure prophylaxis for prevention of HIV varied 
between facilities. Participants raised issues relating to the impact of caring for rape survivors on health care providers, and identified a lack of psychosocial therapy for survivors and many of those who care for them. There are still challenges in providing quality care to rape survivors due to lack of resources and lack of support from non-CFMS staff. This results in other needs of rape survivors continuing to be unmet.

Key words: Sexaul Assualt and Policy implemetation

\section{EPS-0-9: LONG ACTING REVERSIBLE CONTRACEPTION: FACILITY DATA MAPPING TO UNDERSTAND UTILIZATION AND HEALTH PROGRAMME IMPLEMENTATION}

Diantha Pillay', Chelsea Morroni², Melanie Pleaner ${ }^{3}$, Ntombizethu Dumakude 3 , Nicolette Naidoo ${ }^{3}$, Saiqa Mullick ${ }^{3}$

ISchool of Clinical Medicine, Wits Reproductive Health and HIV Institute (WRHI), 'University of Botswana, ${ }^{3}$ University of the Witwatersrand

Implanon NXT, a contraceptive implant which suppresses ovulation for 3 years with pregnancy rates below 1\%, launched in South Africa in February 2014. Anecdotal reports suggest increased early removals and decreased uptake with gaps in literature regarding implementation and reporting. We therefore aimed to determine how implant insertions and removals are recorded and challenges with delivery. Four high and 3 low volume insertion clinics were selected in the City of Johannesburg and Dr. Kenneth Kaunda District. Clinics were scored using a 6-item checklist for data adequacy of elements specific to implant insertions and removals. Informal discussion were scheduled with healthcare providers to understand data collection processes and challenges. Findings revealed that insertions and removals are recorded using homemade tools, with reasons for removal not consistently recorded, leading to underreporting. Six of 7 facilities did not have the National data collection tool for recording implant insertion, removal and adverse reactions, (Active Surveillance Reporting Form for Sub-Dermal Implants). Healthcare workers stated that they had not been oriented about data collection and reporting therefore adopted local solutions and were performing removals under 6 months after insertion due to partner dissatisfaction, side effects or pregnancy. They also highlighted that a limited number of staff were trained on insertions and removals creating a barrier to scaling up the programme. For data to inform effective decision making in the roll-out of Implanon NXT, standardized recording and reporting guidelines, strengthened communication, reinforced practice and increased coverage of training to capacitate relevant cadres of health personnel are needed.

Key words: Contraception, Implanon NXT, programme implementation

\section{EPS-O-10: HOME ENVIRONMENT AND INFANT SURVIVAL: A CALL FOR REPOSITIONING PRIMARY HEALTHCARE IN NIGERIA}

'Bola Lukman Solanke, ${ }^{2}$ Bankole Taofik Olatunji

'School of Social Sciences, Department of Demography and Population Studies, ${ }^{2} O$ bafemi Awolowo University, Nigeria

Survey evidence across developing countries confirms that the home environmental condition is associated with infant survival. However, studies in Nigeria have rarely link directly household environmental factors and infant survival. This study examines the relationship between household environmental factors and infant survival in Northern Nigeria where maternal and child health is poorest in the country. Information about 19,839 women aged 15-49 years and 1,965 recent live births were extracted from 2013 Nigeria Demographic and Health Survey (NDHS). The response variable is infant status at the end of first year. The explanatory variables are household cooking fuel, toilet facility and source of drinking water. The Survival analysis Cox regression was applied. Results show that $64.3 \%$ of the household had poor toilet facility; $91.3 \%$ used high smoke emission cooking fuel; $48.1 \%$ used unsafe drinking water; and $14.3 \%$ of the infants died. Result confirm that household environmental factors contributed to infant deaths $(p<0.05)$. Household environmental factors are significant predictors of infant survival. Repositioning Primary healthcare delivery in Nigeria will not only strengthen health delivery in Nigeria, but also improve households and community health.

Key words: Home, environment, infant, health

\section{EPS-O-11:UNDERSTANDING OF MENTAL HEALTH POLICY AND IMPLEMENTATION NEEDS IN SOUTH AFRICA}

Bekwa Makaula

Department of Psychology, University of the Witwatersrand

Mental illnesses are prevalent on a significant proportion of South African population. In South Africa a number of barriers to mental health care exist due to poor sociopolitical conditions. South Africa has made significant progress in the development of mental health policy, though there have been a number of challenges at the level of implementation. Implementation of policies begins from the recognition that policies cannot be understood in isolation from the means of their execution. Little attention has been paid to the potential role of the mental health system and those working in it. Moreover, little is known of the difficulties encountered in practice and application of the policy as it stands, or its application to current service organisation and resources. In this study semi structured interviews will be administered to 15 facility managers to compare service planning, implementation 
and impact, to get an in-depth understanding of the policy implementation procedures and programme effects. On the face of policy implementation there is a demand for research methods that are tailored to South African context specific policy implementation needs, moreover there is also a need for more mental health system research that is implementation oriented to close the gap of limited research in policy implementation. Hence the proposed study aim's to bring about a paradigm shift in understanding mental health policy implementation priority needs so that holistic and feasible strategies could be explored by mental health care practitioners, academics and policy-makers through research, programme design and policy implementation.

Key words: Mental health - policy - implementation South Africa - research

\section{EPS-O-12: FINDINGS FROM A PROCESS EVALUATION OF AN INTERVENTION USING LAY HEALTH WORKERS TO SUPPORT PRIMARY CARE HYPERTENSION MANAGEMENT IN AGINCOURT, BUSHBUCKRIDGE.}

Felix Limbani', Jane Goudge ${ }^{3}$, Margaret Thorogood², Francesc Xavier Gómez-Olivé ${ }^{3}$

ISchool of Public Health, Department of Centre for Health Policy, Rural Public Health and Health Transitions Research Unit (Agincourt), University of the Witwatersrand, ${ }^{2}$ Warwick University, 3University of the Witwatersrand

Nearly half of adults in the MRC/Wits Agincourt study area are hypertensive, but only $9 \%$ have the blood pressure well controlled. A randomized trial is testing whether lay health workers (LHW), working in the clinics can improve management of hypertension. In my PhD, I am using process evaluation to explain how context and mechanism affected the impact of the LHWs. Data were collected through clinic observations, interviews as well as implementer and researcher diaries. Before the intervention, implementation of the Integrated Chronic Disease Management (ICDM) model varied across clinics. Almost half did not retrieve patients' records prior to appointment; designated consultation rooms and vital signs stations were not always available; and the booking system was erratic. The intervention recruited a professional nurse as implementation manager. LHWs had background in community health work, were residents of villages served by respective clinics and had attained grade 12. Training of LHWs was standard across the clinics, but each clinic adapted the tasks of the LHW to suit its context. During implementation, clinic specific factors affected care of patients with hypertension: constant breakdown of electronic blood pressure machines; rapidly increasing demand for chronic disease care; and varying quality of clinic management. LHWs had positive effects: better booking system; prepacking of medication happening and files pre-retrieved and re-filed after consultation. Providing effective care to the growing numbers of chronic patients is a challenge, particularly with poor maintenance of equipment and insufficient nurses. Clinic re-organization in ICDM requires additional resources, and LHWs can provide valuable support.

Key words: Process Evaluation, Lay Health Worker, Clinic Intervention, Hypertension

\section{EPS-O-13: CHALLENGES OF PUBLIC PRIVATE PARTNERSHIPS IN PRIMARY HEALTHCARE IMPLEMENTATION IN SOUTH AFRICA}

Linda Tebogo Hlabangana', ${ }^{2}$, Drikus Kriek ${ }^{2}$

'School of Clinical Medicine, Department of Diagnostic Radiology, University of the Witwatersrand, ${ }^{2}$ Wits Business School, University of the Witwatersrand

The public health sector in South Africa is increasingly strained by its obligation to serve over 80 per cent of the population on an ever-decreasing government budget. Furthermore, healthcare is neither equitably distributed nor accessible to all in this country. Although primary healthcare (PHC) is central to Government's plans to transform the health services in South Africa, institutions in the public health sector have failed to effectively implement PHC policies as a direct result of poor governance, failures in stewardship and inefficient management. Public-private partnerships (PPPs) may provide the necessary capital and managerial expertise required to successfully implement a national PHC program. This research aimed to perform a stakeholder analysis to investigate the current perceptions and perceived challenges of the role of PPPs in implementing $\mathrm{PHC}$ in South Africa. Semi-structured interviews were used to gather information from seven interviewees, comprising representatives from public and private healthcare and academia. Content analysis of responses found that PPPs could play a significant role in establishing $\mathrm{PHC}$ in South Africa. The research revealed that there is no ideal model for PPPs in PHC and the type of model used should be influenced by the needs of individual communities. The analysis indicated that the burden of HIV/AIDS and upsurge of lifestyle-related diseases have exerted pressure on an already resource-constrained system. The respondents identified the following critical success factors for PPPs to facilitate PHC implementation: government support, good partnership design, clear role definition, managerial competence, trust/transparency and community involvement from project inception.

Key words: Public Private Partnerships, Primary Health Care, South Africa, Challenges 


\section{EPS-O-14: HEALTHCARE PROGRAMMES FOR TRUCK DRIVERS IN SUB-SAHARAN AFRICA: A SYSTEMATIC REVIEW AND META-ANALYSIS}

Samantha Lalla-Edward', Siyabulela Christopher Fobosi', Catherine A Hankins ${ }^{2,4}$, Kelsey K Case ${ }^{3}$, Francois Ventor', Gabriela B Gomez ${ }^{2,5}$

ISchool of Clinical Medicine, Wits Reproductive Health and HIV Institute (WRHI), University of the Witwatersrand, Johannesburg, South Africa, ${ }^{2}$ Department of Global Health/Amsterdam Institute for Global Health and Development, Academic Medical Centre, University of Amsterdam, The Netherlands, ${ }^{3}$ Department of Infectious Disease Epidemiology, Imperial College London, UK, ${ }^{4}$ Department of Infectious Disease Epidemiology, London School of Hygiene and Tropical Medicine, UK, ${ }^{5}$ Department of Global Health, London School of Hygiene and Tropical Medicine, UK

Planning for effective care of truck drivers (TD) is hindered by knowledge gaps about their health needs and the impact of programmes on their health outcomes. We reviewed healthcare programmes implemented for SSA $T D$, assessed the evaluation methods employed, and examined impact on health outcomes. Published and unpublished works from various databases, online search engines, experts and organisations, describing healthcare programmes for TD in Sub-Saharan Africa (SSA) were reviewed. Forest plots of impact and outcome indicators with unadjusted RR and $95 \% \mathrm{Cl}$ were created. Subgroup analysis by indicator type using a random-effects model to assess between-study heterogeneity was performed. Sensitivity analysis to examine the effect estimate chosen (risk difference instead of RR) and model to summarise results (fixed vs. random effects) was conducted. Thirty seven publications describing 22 healthcare programmes across 30 countries were included from 5,599 unique records. All programmes had a HIV-prevention focus with three describing primary healthcare services. Twelve evaluations assessed changes in input, output, and outcome indicators. Absence of comparison groups, preventing attribution of the effect observed to the programme and lack of biologically confirmed outcomes were the main limitations. Four programmes estimated a quantitative change in HIV prevalence/reported STI incidence, with mixed results. Programmes showed positive changes in risk behaviours, knowledge, and attitudes. Results were robust in sensitivity analyses. Diverse healthcare programmes tailored to TD needs implemented in 30 SSA countries showed potential benefits. Information gaps about the availability and quality of services provided and their effects impede further planning and implementation of evidence-informed effective health programmes for TD.

Key words: Truck drivers, evaluation, healthcare, intervention

\section{EPS-O-15: MARKET ENTRY BARRIERS FOR HIV SELF-TESTING IN SOUTH AFRICA}

Mohammed Majam, Rasaova Rijamampianina

Business School, Wits Reproductive Health and HIV Institute (WRHI), University of the Witwatersrand

South Africa faces greatest burden of the HIV epidemic worldwide. Even though significant progress has been made to improve testing rates, there still remains a significant testing gap. HIV Self-Testing (HIVST) using Rapid Diagnostic Technology is a novel, innovative approach that can potentially be disruptive to the industry. Currently, no legislation, regulation or statutes exist that govern the sale and use of HIVST in SA. HIVST are currently being sold in several countries worldwide, however, there are currently no device manufacturers that are willing to transact in South Africa. This study examines the structural and strategic barriers to market entry that the device manufacturers believe exist, whilst also interrogating the role and extent to which statutory barriers inhibit market entry. The study uses data collected through semistructured interviews with representatives of 15 participants in the sector, including device manufacturers, $\mathrm{NDOH}$, the WHO, Key Opinion Leaders and technical experts. The study found that the greatest barrier to market entry is the uncertainty regarding the regulatory pathway prior to market entry. Absence of clear policies from the SA $\mathrm{NDOH}$ and normative guidance from the WHO has made it an investment risk to enter the SA market. Key opinion leaders believe that HIVST has the potential to change the market, however, needs to be done correctly, taking into consideration several socio and economic considerations. The study concludes that, on balance, conditions for entering the SA market are cogent on the development of a clear regulatory pathway and explicit acceptance of the concept by government.

Key words: HIV Self-Testing, Policy

\section{EPS-O-16: MOBILISING COMMUNITIES AND CONSCIENTISING MEN FOR 'SUSTAINABLE' CHANGE: AT THE INTERSECTION OF HEALTH POLICY, FEMINISM AND INTERNATIONAL DEVELOPMENT}

Ruari-Santiago McBride

Post-doctoral research fellow, Centre for Health Policy, School of Public Health, University of Witwatersrand

Globally, it is estimated that one in three woman have been subjected to some form of physical and/or sexual violence by a man. Violence against women and girls (VAWG) has historically been theorised by feminists as a manifestation of patriarchy that is perpetuated by men's adherence to hegemonic gender norms. More recently, VAWG has been re-framed as a public health problem, which results in poor health for individual women, and a matter of international development, which impedes 
economic and social progress. This shift has generated new imperatives, and funding streams, to determine the most effective health policies to reduce VAWG. The UK's Department of International Development (DIFD), for example, launched the 'What Works' programme in 2014, which provided $£ 18 m$ to 17 research projects across 14 different countries, in order to answer the question: how can we prevent VAWG sustainably across the globe? This presentation will provide preliminary findings from an ethnographic process evaluation of one these 17 research projects: the Change Trial, a community mobilisation intervention being implemented in a South African 'township'. I will investigate the experiences of the men targeted by this public health intervention, the community members implementing it and the academics and NGO staff managing it in order to better understand the processes and mechanisms of mobilising communities and conscientising men. Adopting a critical perspective I will analyse the local frictions and global connections that emerge from attempts to 'sustainably' reduce VAWG.

Key words: gender based violence, feminism, global health policy, international development

\section{EPS-O-17: FEMALE SEX WORKERS' EXPERIENCES IN ACCESSING HIV AND SRH SERVICES, IN KWAZULU-NATAL, SOUTH AFRICA}

Letitia Rambally-Greener, Ross Greener, Mags Beksinska, Melanie Drace, Kedibone Sithole, Muriel Kubeka, Jennifer A Smit

School of Clinical Medicine, Department of Obstetrics and Gynaecology, MatCH Research Unit (MRU), Department of Obstetrics \& Gynaecology, University of the Witwatersrand, Durban, South Africa

Female sex workers (FSWs) have an increased risk for HIV acquisition and sexually transmitted infections. Their vulnerability is largely attributed to sexual practices, but is compounded by their experiences of prejudice, stigma and discrimination. We explored barriers to healthcare service access and avenues for improvement, from the perspective of FSWs. Data were collected in 2012 as part of a five year, multi-country project seeking to enhance HIV, sexual and reproductive health services for FSWs. Four semi-structured focus groups were conducted with 35 FSWs in isizulu and English in the eThekwini District, exploring barriers or facilitators to accessing healthcare. Inductive content analysis were conducted on the data. Participants' mean age was 28 and most began sex work at 22 years. Worked from a combination of streets $(35 \%)$, bars $(28 \%)$, hotels $(22 \%)$ or homes $(7 \%)$. Most required HIV testing, family planning and condoms and reported numerous barriers to healthcare, such as, refusal or poor care, inability to access more than one service per visit, difficulty accessing condoms, and discrimination from providers when they disclose or are identified as FSWs. FSWs strongly felt that provider attitudes must be addressed: "...we must be with everyone, be together with everyone but they [providers] only have to change the way they work or behave". FSW' reported difficulty accessing services despite needing them and those who managed to access service reported negative experiences. More innovative interventions are needed to adequately address these needs; through peer driven outreach and healthcare worker sensitisation.

Key words: Female sex workers, barriers, sexually transmitted infections

\section{EPS-0-18: STAKEHOLDER'S UNDERSTANDINGS OF BEING PART OF A HEALTH AND SOCIO-DEMOGRAPHIC SURVEILLANCE SITE IN RURAL SOUTH AFRICA}

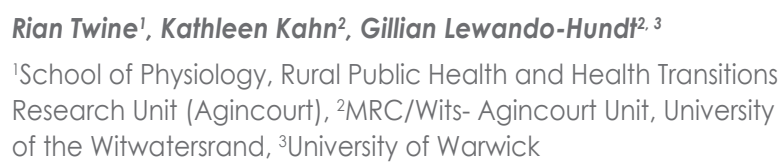

Longitudinal study designs, such as the MRC/WitsAgincourt health and socio-demographic surveillance system (HDSS), involve long-term involvement of study communities with researchers. Unlike a time-limited cross-sectional study, longitudinal health research in geographically defined areas involve the participants, users of research information, university, researchers, and service providers such as municipalities, Departments of Health and Education, in ongoing interactive relationships. The Agincourt HDSS site, started in 1992, aims to highlight health needs in transitioning rural populations, information essential in the evaluation of health services, and provides an invaluable contribution to the development of appropriate health policies. This paper will present the results of a study conducted from August 2015 to March 2016, where individual interviews and focus group discussions were held with ward councilors, indunas, municipal managers, district health officials, Community Development Forums and home based carers. Their views were sought on the benefits and challenges for individuals, households and community leadership of being part of a health and socio-demographic surveillance site. Results will highlight the experience, knowledge and understanding of community leaders, public sector stakeholders and political leaders concerning participating in research activities in a longitudinal health surveillance study site. This paper will develop the concept of participatory governance of research in the local/global context and the interface between universities and communities in which they conduct research. The discussion will draw on the changing political and social environment in the Agincourt HDSS and identify strategies and methods of participatory governance and public engagement.

Key words: Longitudinal research, stakeholder engagement 


\section{ESP-P-1: KNOWLEDGE AND THE SOURCES OF KNOWLEDGE OF SPINAL ANAESTHESIA IN PRIMIPAROUS WOMEN WHO HAVE RECEIVED A CAESAREAN SECTION}

Carien Moller, Helen Perrie

School of Clinical Medicine, Department of Anaesthesiology, University of the Witwatersrand

Very little is known about the knowledge that South African women have concerning labour and anaesthesia, particularly spinal anaesthesia for caesarean section, and also which sources they can access to obtain this information. The aim of this study was to describe the knowledge and the sources of knowledge of spinal anaesthesia in primiparous women who had received a caesarean section at Chris Hani Baragwanath Academic Hospital. A prospective, contextual, cross-sectional descriptive research design was used. A self-administered questionnaire was developed and a convenience sampling method was used to enrol 86 primiparous women over a 13 month period. Women's scores for level of knowledge ranged from $3(20 \%)$ to $13(86.67 \%)$ out of 15. The mean score out of 15 was 7.84 (SD 2.12) which is $53 \%$. When asked to choose a source of knowledge of spinal anaesthesia before admission to hospital, most women selected no information, 25 (29.07\%), midwives, 13 (15.12\%), and family and friends, 11 (12.79\%). After admission to hospital, the anaesthetist, 29 (33.72\%), the midwife, 18 (20.93\%), and no information, 10 (11.63\%) were the most commonly selected options. From this study it can be concluded that primiparous women have a limited knowledge of spinal anaesthesia when presenting for caesarean section. Women often rely on "non-medical" sources of information, but midwives and anaesthetists are still common sources of information. Medical professionals are therefore ideally placed to improve women's knowledge of spinal anaesthesia.

Key words: Knowledge, spinal anaesthesia

\section{ESP-P-2: AUTHENTIC PATIENT ENGAGEMENT THROUGH EXPERIENCE- BASED CO-DESIGN (EBCD)}

\section{Claire van Deventer}

School of Clinical Medicine, Department of Family Medicine and Primary Care, University of the Witwatersrand, Medical School

In 2010, it was found that $14 \%$ of children admitted in a hospital in the North West Province, South Africa, were severely malnourished. More than $70 \%$ of these children were also HIV positive. A quality improvement plan (QIP) with a number of interventions was initiated. However, patients were not involved and this was later considered to be an extra option to improve outcomes, adding value to the ongoing QIP. Experience based co-design is an approach that goes beyond patient satisfaction or patient perceptions of quality in health care and attempts to add the dimension of patients' feelings about the service provided. Using a process of observation of touchpoint areas, audiotaped interviews with staff, videotaped interviews with parents of ill children and feedback meetings, a co-designed quality improvement process was set in motion to improve the services for ill children across all areas in the Potchefstroom sub-district. Seven main themes outlined staff's perception of care at all touch points and three main themes were extracted from parent interviews. Identified and prioritised issues will be presented, together with a demonstration of the whole process. Co-design of a paediatric services is possible when all role-players are involved as quality improvement members

Key words: Experience-based co-design, Children, QIP

\section{ESP-P-3: A QUALITY IMPROVEMENT PROJECT REGARDING HIV MANAGEMENT IN CHILDREN IN PRIMARY HEALTH CARE}

\author{
Claire van Deventer
}

School of Clinical Medicine, Department of Family Medicine and Primary Care, University of the Witwatersrand, Medical School

In Dr Kenneth Kaunda district (DKK), North West province, children diagnosed with HIV have been downreferred from hospitals to primary care over the last number of years to ensure accessibility of services and to decrease defaulting due to socio-economic causes. The on-going care of these children was a concern to the District Clinical Specialist Team (DCST) in this district. For this reason a quality improvement project was embarked upon in 2014. To identify the current clinical care for children on ART at PHC clinics in DKK and to plan interventions to improve such care. An initial audit was done followed by a team-led intervention based on a standardised approach/tool to each child in PHC. This was allocated to individual PHC doctors in an attempt to make them champions of the project. The initial audit indicated poor performance of the most important indicators eg the district wide viral load suppression was $66.6 \%$, blood tests were being done inadequately, prophylactic INH was not being given and there was no standard approach across the district to these children. The intervention with a targeted tool to check the contents of each file and the proactive management of children failing treatment will be discussed together with the results of the above audit. General measures eg training of all doctors and nurses do not necessarily translate into better care of a complex condition eg paediatric HIV. A targeted intervention however can make a significant difference.

Key words: QIP, child ART, PHC 
ESP-P-4: STRATEGIC DIRECTIONS FOR 90-90-90: PERFORMANCE OF HIV PATIENT MANAGEMENT AT FACILITIES IN GAUTENG AND NORTH WEST PROVINCE BETWEEN $2006-2008$

Diantha Pillay, Nicolette Prea Naidoo, Samanta Tresha Lalla-Edward

School of Clinical Medicine, Wits Reproductive Health and HIV Institute (WRHI), University of the Witwatersrand

The UNAIDS 90-90-90 goal was implemented at public health facilities in South Africa which aims to test $90 \%$ of the population for HIV, treat $90 \%$ eligible to receive antiretroviral treatment (ART) and have $90 \%$ of the infected population virologically suppressed by 2020. Retrospectively assessing performance of HIV patient management at facilities is beneficial to inform strategic direction in achieving these goals. From 2006 to 2008 RHI conducted file reviews at 8 ART sites in Gauteng and North West provinces reviewing over 30,000 patient files. The aim was to collate and analyze data and provide information on facility performance and patient clinical status as a basis for evidence-based treatment, decision making and change ideas.Findings revealed that more females $(65 \%)$ accessed the facility than males (35\%). Almost half $(45 \%)$ of facility attendees had baseline viral loads over 100000 copies of virus $/ \mathrm{mL}$. In most sites patients were presenting with CD4 counts of 90-130 cells/mm. Viral suppression levels of $13 \%-73 \%$ were seen, high levels of loss to follow up of patients 6 months post-treatment initiation ranging from $24 \%-97 \%$ and ART initiation at all facilities ranged from $87 \%-100 \%$. Results represent deficiencies in HIV testing, linkage to care and need for better patient monitoring. Loss to follow up could reflect inadequacies with data capturing and reporting. To ensure achievement of 90-90F-90 goals focus needs to be on better access to men, linkage and retention in care and strengthened M\&E systems for monitoring patient outcomes and programmatic impact.

Key words: 90-90-90, HIV testing, viral suppression

\section{ESP-P-5: FACTORS ASSOCIATED WITH SURVIVAL TO DISCHARGE OF NEWBORNS IN A MIDDLE INCOME COUNTRY.}

\author{
Daynia Ballot
}

School of Clinical Medicine, Department of Paediatrics, University of the Witwatersrand

Ongoing clinical and survival audit is an essential part of quality improvement in health care. Review neonatal care and predictors of short term survival in neonates at a tertiary hospital in Johannesburg, South Africa. Review of an existing neonatal database, including neonates weighing > 400 grams admitted within 48 hours of age between 1 January 2013 and 31 December 2015. A total of 5018 neonates were included in the review.
The mean birth weight was 2148 grams (Standard Deviation [SD]: 972) and mean gestational age was 34.2 weeks (SD: 4.8). Overall survival was $85.6 \%$ (4294/5018). The most common causes of death were prematurity (46.2\%), hypoxia (19.5\%) and infection (17.2). Most deaths occurred in VLBW neonates $479 / 724$ (66.1\%). The strongest predictors of survival were birth weight (OR $1.0 ; 95 \% \mathrm{Cl} 1.0$ - 1.01) and gestational age (OR=1.1, 95\%Cl: 1.05 - 1.17). Other predictors of survival included metabolic acidosis (OR=0.14, 95\% Cl: $0.09-0.20$ ), hyperglycemia (OR=0.31, $95 \% \mathrm{Cl}: 0.23-0.41)$, mechanical ventilation $(\mathrm{OR}=0.35$, $95 \% \mathrm{Cl}: 0.28-0.46)$, major birth defect $(\mathrm{OR}=0.12,95 \% \mathrm{Cl}$ : $0.08-0.18)$, resuscitation at birth $(\mathrm{OR}=0.39,95 \% \mathrm{Cl}: 0.31-$ 0.49 ) and Caesarean section (OR=1.8, 95\% Cl: $1.44-2.25)$. Resources need to be focused on improved care of VLBW infants.

Key words: Neonates, survival, preterm neonates

\section{ESP-P-6: INTRODUCING BLENDED LEARNING ON THE USE OF A PARTOGRAM INTO THE 3RD YEAR MEDICAL STUDENTS OBSTETRICS BLOCK: A RANDOMIZED QUASI-EXPERIMENTAL STUDY}

Hennie Lombaard', Mubuuke Gonzaga'2, Wilma De Witt ${ }^{3}$

'School of Clinical Medicine, Department of Obstetrics and

Gynecology, ${ }^{2}$ Makerere University, ${ }^{3}$ University of Pretoria

Literature shows that blended learning is as good as or even better than didactic learning. There is a dearth of literature on blended learning in obstetrics. The objective of this study was to explore students' perceptions and experiences of using a blended learning approach in obstetrics. This was a mixed methods study with a quasi-experimental design. The study population was 3rd year medical students at the University of Pretoria. The group was of 282 students. Half the group was randomized to receiving didactic learning and half blended learning. A questionnaire and two foucus groups were used. A 10 mark question on filling of the partogram in osce format was used. The Mann Whitney U test was used for categorical data and the student T-test for linear data. The total questionnaires answered were 106 out of 141 (75\%) didactic learning and 116 out of 141 (82\%) in the blended learning group. There was only a significant difference between the groups in the following questions in terms of ability to reach specific learning needs ( $p$ value $=0.00244$ ) and the access to teaching material ( $p$ value $=0.0001$ ) favoring blended learning. The students in the blended learning group did significantly better in the test, $p$ value $<0.00001$.

Conclusion: Students feel that there is a need for blended learning in the teaching of the partogram. They feel that it better allowed them to reach their specific learning needs and it was easier to access.

Key words: Blended learning 


\section{ESP-P-7: THE ACCURACY OF NURSE PERFORMANCE OF THE TRIAGE PROCESS IN AN ACADEMIC EMERGENCY DEPARTMENT IN GAUTENG}

Kaamil Alli', Kaasifa Gathoo, Lara Goldstein ${ }^{2,3}$, Mothusi Mothopeng ${ }^{3}$, Tasnim Sallie ${ }^{3}$, Fatima Samodien ${ }^{3}$

'Department of Family Medicine, University of the Witwatersrand, ${ }^{2}$ Helen Joseph Hospital, ${ }^{3}$ Wits University

Triage in the Emergency Department (ED) is required in order to prioritise relatively scarce resources according to the acuity of patient illness or injury. The South African Triage Scale (SATS) is a well-validated method of performing triage in the South African setting. In this study, we did not assess the accuracy of the SATS tool itself, but rather the accuracy of its usage by nurses in the ED. Hence, the traditional terms of over- and under-triage could not be used but rather the terms promotion and demotion respectively. No other studies have made use of these terms when referring to triage performance accuracy. A total of 1091 patient triage forms were reviewed. Triage was correctly performed in $65 \%$ of patients. Of the $30 \%$ where the triage was performed incorrectly, $44.4 \%$ of patients were falsely promoted and $55.6 \%$ of patients were falsely demoted. However, of those incorrectly categorised, approximately half were still triaged correctly despite the error. The most common reason for erroneous promotion or demotion was related to the improper usage of the triage discriminator. Triage quality assurance needs to be supported by regular refresher training of the triage procedure with a focus on common error areas especially discriminator usage.

Key words: Triage, Emergency, Accuracy, SATS

\section{ESP-P-8: COMMUNITY MENTAL HEALTH SERVICES IN SOUTHERN GAUTENG: A POLICY IN NEED OF IMPLEMENTATION}

\author{
Lesley Robertson, Christopher Szabo
}

School of Clinical Medicine, Department of Psychiatry, University of the Witwatersrand

Over the past 15 years South Africa has introduced new health and mental health legislation and policy. However, the National Mental Health Policy (NMHP) has not been incorporated into the National Health Strategic Plan or the $\mathrm{NHI}$ white paper. The aim of this study was to analyse the community mental health services (CMHS) in the Southern Gauteng region of South Africa in the light of the system and resources proposed by the NMHP. The principal objective was to advance the implementation of policy through improved understanding of the current situation. We used data and information which was available to the Department of Health for planning purposes. Data regarding clinic services was drawn from that collected routinely by the Gauteng District Health Information Services for the year 2014/2015. Supplementary information regarding staffing and facilities was obtained via the
Gauteng Directorate of Mental Health. Using descriptive analysis, we found the human resources and the mental health coverage of the population to be much lower than that recommended by the NMHP. Our findings also suggest that the CMHS, positioned as a primary health care programme, could be compromised by primary health care re-engineering and the implementation of the integrated chronic disease management programme. Our recommendations include the full incorporation of the NMHP into national health strategic plans, the establishment of a ring-fenced mental health budget within $\mathrm{NHI}$, and continued development of primary mental health care with specialist support from adequately staffed CMHS. However, active leadership is needed to ensure these recommendations are addressed.

Key words: Policy implementation, mental health

\section{ESP-P-9: ADEQUACY OF POST CAESAREAN SECTION PAIN MANAGEMENT AT THE TIME OF DISCHARGE AT AN ACADEMIC HOSPITAL}

Makhosazana Dlamini', Juan Scribante ${ }^{2}$, Helen Perrie ${ }^{2}$, Sean Chetty ${ }^{3}$

'School of Clinical Medicine, Department of Anaesthesiology, University of the Witwatersrand, Anaesthesiology, ${ }^{2}$ Wits Research Unit, ${ }^{3}$ HOD Rhahima Moosa Mother and Child Hospital, Special Anaesthesiologist

Adequate analgesia post caesarean section accelerates ambulation, improves patient outcome, reduces maternal morbidity, and facilitates early infant care. Clinical practice guidelines for postoperative caesarean section pain management, if successfully implemented and adhered to, should improve quality of care and patient pain outcome. Current trends are moving towards earlier discharge of patients post caesarean section. The aim of this study was to determine whether guidelines used for post caesarean section pain management adequately controlled pain at the time of discharge which was 48 hours post operatively at Chris Hani Baragwanath Academic Hospital. This was a prospective, contextual, descriptive study. Convenience sampling was used and 91 patients were enrolled after informed consent was obtained. At discharge patients pain was measured using a Visual Analogue Scale score and the patients' pain management was documented. The mean length of stay post caesarean section was 43.48 (SD 7.52) hours, with a minimum of 29 and a maximum of 66 hours. Of the 91 patients, $54(59.34 \%)$ patients had a score $<40 \mathrm{~mm}$, indicating adequate pain control and 37 (40.66\%) patients had a score of $>40 \mathrm{~mm}$, indicating inadequate pain control. The departmental pain management guidelines were correctly prescribed for all patients, however none of the patients received pain management as recommended by the guidelines. In this study the maintay of pain management was Omnopon. At the time of discharge, more than half of the patients had adequate 
pain control despite receiving less pain medication. Education regarding pain management could result in patients receiving better pain management.

Key words: Pain, Caesarean Section

\section{ESP-P-10: UPTAKE OF HIV COUNSELING AND TESTING IN A MOBILE CLINIC PROGRAMME WITHIN THE INNER CITY OF JOHANNESBURG: A CROSS-SECTIONAL STUDY}

\section{Patrick Ngassa Piotie}

Wits Reproductive Health and HIV Institute (WRHI), University of the Witwatersrand

The success of the newly adopted UNAIDS 90-9090 strategy relies heavily on accessible HCT services. Numerous studies conducted in sub-Saharan Africa have shown that mobile services increase uptake of HCT. The aim of this study was to describe the service delivery model implemented by the Wits RHI Mobile Clinic Programme and the characteristics of the clients who accessed the service. This was a retrospective cross-sectional study in the inner City of Johannesburg. HCT was offered to clients along with other disease screening services. Demographic and service utilisation data were routinely collected from clients who visited the mobile clinics between January 2013 and December 2015. Descriptive statistics were calculated. During the study period, 4557 clients visited the mobile clinic. Clients were predominantly males $(55 \%)$ and black (83\%). The mean age was $31.2( \pm 10.3)$ years old, ranging between 13 and 85 . Adolescents (13-19 years) and young adults (18-32 years) represented $63.7 \%$ of the study population. HCT was offered to $94 \%$ of clients with $7 \%$ (273/3857) testing HIV-positive. Approximately $39 \%$ of clients were either first time testers or had their last HIV test more than 12 months ago. Linkage to care was facilitated for patients requiring ART. The Wits RHI mobile clinics provides a great opportunity to increase HCT uptake, to find first-time testers and to access those populations who do not typically access services at fixed clinics i.e. males, adolescents and young adults. To ensure continuity of care, linkage to care systems must be strengthened.

Key words: HIV testing, mobile clinics

\section{ESP-P-11: A COMMUNITY HEALTH WORKER TO IMPROVE ADHERENCE TO ANTI-EPILEPTIC DRUGS IN RURAL SUB- SAHARAN AFRICA: IS IT COST-EFFECTIVE?}

Ryan Wagner', Fredrik Norström², Stephen Tollman³, Charles Newton ${ }^{4}$, Melanie Bertram ${ }^{4}$, Lars Forsgren ${ }^{2}$, Lars Lindholm²

'School of Public Health, Rural Public Health and Health Transitions Research Unit (Agincourt), 2Umeå University, ${ }^{3}$ University of the Witwatersrand, ${ }^{4}$ Oxford University, ${ }^{5}$ World Health Organization
Epilepsy is a common, chronic neurological disorder that disproportionately affects individuals living in low- and middle-income countries (LMICs). Whilst anti-epileptic drugs (AEDs) can reduce or eliminate seizures, the epilepsy treatment gap remains high and adherence to AEDs remains low in LMICs. Using community health workers (CHWs) has been shown effective at improving adherence for other chronic medications, yet no study exploring the use of $\mathrm{CHWs}$ for improving adherence to AEDs has been conducted in LMICs. The objective of the study was to assess the cost effectiveness of working with community health workers to improve adherence to anti-epileptic drugs in rural South Africa. We designed a Markov model with age- and sex-varying transition probabilities to determine whether introducing a $\mathrm{CHW}$ to improve adherence to AEDs is cost-effective. Data were derived using published studies from the Agincourt subdistrict in rural northeastern South Africa. Cost and health state values were derived from government figures and international disability weights, respectively, and utility was measured using quality adjust life years (QALYS).The intervention cost 664,320 ZAR per annum per sub-district. The intervention cost 8,053 ZAR and 10,009 ZAR per QALY gained for males and females, respectively. Increasing the cost of the intervention and reducing the effectiveness resulted in figures still less than the cost-effectiveness threshold.CHWs were found to be cost-effective and the intervention dominated even when the costs and effects of the intervention were varied. The primary health care reengineering currently underway in South Africa identifies CHWs as a vital link in primary health care. Introducing CHWs to visit people with epilepsy is likely a cost-effective intervention that would reduce the burden of epilepsy in rural South Africa.

Key words: Epilepsy, economic evaluation, community health worker, adherence

\section{ESP-P-12: CCESS AND UTILISATION OF HEALTHCARE SERVICES BY SEX WORKERS IN SOUTH AFRICA: NORTH STAR ALLIANCE AS A CASE STUDY}

Samantha Lalla-Edward', Siyabulela Christopher Fobosi' , Edwin Mkhwanazi', Paul Matthews'2, Catherine Hankins ${ }^{3,4}$, Eugene Sickle', Francois Venter', Gabriela Gomez ${ }^{3,5}$

'School of Clinical Medicine, Wits Reprodutive Health and HIV Institute (WRHI), University of the Witwatersrand, South Africa, ${ }^{2}$ North Star Alliance, South Africa, ${ }^{3}$ Amsterdam Institute for Global Health and Development, Department of Global Health, Academic Medical Center, University of Amsterdam, The Netherlands, "4Department of Infectious Disease Epidemiology, London School of Hygiene and Tropical Medicine, UK,

${ }^{5}$ Department of Global Health and Development, London School of Hygiene and Tropical Medicine, UK

Sex-worker specific health services aim to respond to the challenges that this population faces in accessing healthcare. North Star Alliance (NSA) is a public-privatepartnership providing care to hard-to-reach populations. 
Services include condom distribution, STI treatment, HIV testing and counselling (HTC), tuberculosis and malaria screening and treatment, family planning, and general primary healthcare (PHC). We describe uptake, utilisation, and female SW(FSW) views of NSA services.Mixed-methods were used:- quantitative analysis(using Excel 2016) of an anonymised subset of routinely-collected clinic data for January 2013 to September 2015. Qualitative analysis (analysed in Nvivo 10) of 25 semi-structured interviews conducted with FSWs accessing five NSA sites.3,770 FSWs made 5,827 healthcare visits across sites(mean: 1.6 visit per person). We observed an annual increase in the number of visits (a $43 \%$ increase was observed between 2014 and 2015 only). FSWs accessed PHC services (36.7\%) followed by HTC(28.9\%). Border sites accounted for the majority of visits. FSWs were young (mean age: 24 to 33 years) and mainly South African (>80\%), except for Musina(Zimbabwean origin: $83.8 \%$ ). FSWs reported satisfaction with location, size and scope of services offered, staff and free services. Revising the opening hours was the single change mentioned to increase access. FSWs accessing sites report available services being suitable and accessible. We observed an increase in service utilisation with demand focusing on HIV prevention and PHC services. From July 2015, NIMART and ART was started in three South African clinics. Treatment uptake has been slow and results will be used to adapt the way services are provided and ultimately improve treatment access.

Key words: Healthcare, access, sex worker

\section{ESP-P-13: TEENAGE PREGNANCY AND RACIAL HETEROGENEITY IN SOUTH AFRICA}

Sibusiso Mkwananzi', Clifford Odimegwu²

'School of Social Sciences and Public Health, Department of Demography and Population Studies, ${ }^{2} \mathrm{HOD}$ Demography and Population Studies

Teenage pregnancy remains a health and social challenge globally and especially in sub-Saharan Africa. Past research has shown factors associated with the phenomenon in South Africa yet these have concentrated on individual-level variables. However, community-level characteristics and particularly the influence of racial heterogeneity $(\mathrm{RH})$ have not been explored. To examine the effect of racial heterogeneity on teenage pregnancy. Data of pregnancy experience among females aged 12 to 19 years were extracted from the 2001 and 2011 censuses. Descriptive statistics and multilevel logistic modeling were performed. We tested the association between racial heterogeneity and teenage prgenancy controlling for age group, marital status, education level, houesehold sex composition, household density, province and community levels of gender equality. As community levels of racial heterogeneity increased the likelihood of teenage pregnancy decreased.In 2001 (Medium Comm. RH levels:0.92; $p<.05$ \& High RH levels:0.87; $p<.05)$ and in 2011 (High Comm. RH levels:0.81;p<.01) community levels of racial heterogeneity were independently associated with teenage pregnancy. These results may be linked to the fact that racial heterogeneity in the country occurs more among higher socio-economic communities. These results assist in the understanding of teenage pregnancy in South Africa.

Key words: Teenage pregnancy, racial heterogeneity, multilevel modelling, social disorganisation theory

\section{ESP-P-14: A CLINICAL AUDIT: DENTAL TREATMENT NEEDS AND TREATMENT RECEIVED BY 12 AND 15 YEAR OLD CHILDREN WHO ATTENDED THE WITS DENTAL HOSPITAL DURING THE PERIOD: JANUARY 2006 TO DECEMBER 2010.}

Tashnim Bagus, J.L Shackleton

School of Oral Sciences, Oral Rehabilitation, Faculty of Health Sciences, University of the Witwatersrand

The majority of children and adults are affected by dental caries and its sequelae in South Africa. Children, from lower socioeconomic backgrounds in particular, continue to experience a high burden of dental caries. The objectives were to determine the profile, dental treatment needs and treatment received by 12 - year - old and of 15 - year - old patients who attended the Wits Dental Hospital during the period of January 2006 to December 2010. Record reviews from January 2006 to December 2010 were done. The mean DMFT for the 12 -year old age group was 3.0. The DT accounted for $76.6 \%$ of the DMFT. The mean DMFT for the 15 - year - old age group was 3.8. The DT accounted for $92.1 \%$ of the DMFT. There is an increase in dental caries from 12 - year - old to 15 - year - old patients. Carious teeth are left untreated. Restorative dental treatment levels are low. Low levels of completed treatment plans noted. The rise in dental caries from the 12 -year - old group to the 15 -year - old group, together with high levels of untreated caries and low levels of restorative dental treatment indicate the need to improve the oral health of school children. Evidence - based oral health prevention and promotion strategies must guide oral health policy planning, so that supportive environments are created and sustained to facilitate healthy choices and maintain optimal oral health.

Key words: Caries, Dental Treatment Needs, Children, Prevalence 


\section{INFECTIOUS DISEASES}

\section{ID-O-1: CHARACTERIZATION OF LYTM- DOMAIN CONTAINING PROTEINS IN MYCOBACTERIUM SMEGMATIS}

Moagi Shaku',2, Bavesh Kana ${ }^{2}$, Melissa Chengalroyen²

'School of Pathology, Faculty of Health Sciences, University of the Witwatersrand, ${ }^{2}$ Centre of Excellence for Biomedical TB Research

Mycobacterium tuberculosis encasesitself within a complex cell wall consisting of mycolic acids, arabinogalactan and peptidoglycan layers. The peptidoglycan is important for structural maintenance and osmotic protection of the cell. Beta-lactam antibiotics such as penicillin can perturb peptidoglycan biogenesis by inhibition of penicillinbinding proteins and cause cell death. However, penicillin insensitive enzymes involved in peptidoglycan biogenesis such as amidases, transglycosylases and endopeptidases remain to be exploited for anti-TB drug development in light of the frequent emergence of drug resistant strains. In this study we functionally characterize the LytMdomain containing endopeptidases of mycobacteria. Bioinformatics tools were used to identify LytM domaincontaining homologues in Mycobacterium smegmatis, (designated MepB1-MepB4). These were deleted using standard allelic exchange mutagenesis and recombination techniques and the resulting mutants were assessed for cell wall related defects. We find that mycobacterial LytM endopeptidases are dispensable for growth but have important roles in cell division as demonstrated by reduced growth kinetics and defective cell shape in a $\triangle m e p B l$ deletion mutant. In addition disruption of mepB3 results in failed cell separation after cytokinesis, a defect that was exacerbated in a $\Delta$ mepB2 $\triangle$ mepB3 double deletion mutant. Spatial localization of new cell wall biosynthesis revealed the inability to degrade the bridge joining two daughter cells, pointing to a critical role of these enzymes in cell separation. Collectively, these observations provide the first insight into a new group of potential drug targets for tuberculosis disease and notably enhance the overall understanding of peptidoglycan turnover in a group of clinically relevant pathogens.

Key words: Mycobacteria, Cell wall, peptidoglycan, M23 peptidases

\section{ID-O-2: IDENTIFICATION AND CHARACTERIZATION OF MYCOBACTERIAL CELL WALL AMIDASES}

Sibusiso Senzani ${ }^{1,2}$, Bavesh Kana ${ }^{2}$, Neeraj Dhar ${ }^{3}$

'School of Pathology, Department of Haematology, University of the Witwatersrand, ${ }^{2}$ DST/NRF Centre of Excellence for Biomedical TB Research, ${ }^{3} \mathrm{Global}$ Health Institute, Ecole Polytechnique Fédérale de Lausanne, Lausanne

The emergence of drug resistant strains of Mycobacterium tuberculosis (Mtb) highlights the need for novel drugs to treat tuberculosis. In this regard, peptidoglycan (PG) associated enzymes constitute attractive drug targets, primarily due to their bacterial specific nature. This study focuses on a group of PG hydrolysing enzymes, termed amidases, which have been linked to a number of essential cellular processes in cell division and the bacterial response to stressful conditions. Our approach involved targeted deletion or depletion of amidaseencoding genes in Mycobacterium smegmatis and Mtb, followed by the phenotypic characterization of mutant strains to assess biological function. We identified and characterized four amidases, designated amil-ami4. We demonstrate that ami2 is essential for growth and further show that depletion of this enzyme results in shortening of cells. Deletion of amil results in chains consisting of a minimum of three cells due to incomplete cell division and the formation of aberrant branches along the longitudinal axis of the cells, in the case of Mycobacterium smegmatis. In Mtb, deletion of amil gave rise to polar cellular budding leading to the production of inert "ghost" cells that failed to sustain a subsequent round of replication. Furthermore, amil deletion resulted in retarded and reduced growth under stress conditions, suggesting a possible role in bacterial tolerance. Our findings ascribe a novel role for amidases in mycobacterial growth, cell separation and tolerance. The essentiality of ami2 and detrimental effects resulting from loss of amil suggests that these enzymes may serve as useful drug targets for tuberculosis disease.

Key words: Amidase, Cell Wall, Cell Division, Mycobacterium 


\section{ID-O-3: ESTIMATION OF SEVERE PNEUMOCOCCAL DISEASE CASES AND DEATHS PREVENTED BY PNEUMOCOCCAL CONJUGATE VACCINE INTRODUCTION IN CHILDREN < 5 YEARS OF AGE IN SOUTH AFRICA IN 2013}

Claire von Mollendorf 1,2, Stefano Tempia ${ }^{3,4}$, Anne von Gottberg 1,5, Susan Meiring ${ }^{6}$, Vanessa Quan', Linda de Gouveia', Charles Feldman ${ }^{7,8}$, Jeane Cloete9, Shabir A. Madhi ${ }^{1,5}$, Katherine L. O'Brien ${ }^{10}$, Keith P. Klugman "1, Cynthia G. Whitney ${ }^{3}$, Cheryl Cohen ${ }^{1,2}$

${ }^{1}$ Centre for Respiratory Diseases and Meningitis, National Institute for Communicable Diseases, a division of the National Health Laboratory Service, Johannesburg, South Africa, ${ }^{2} \mathrm{School}$ of Public Health, Faculty of Health Sciences, University of the Witwatersrand, Johannesburg, South Africa, ${ }^{3}$ National Center for Immunization and Respiratory Diseases, Centers for Disease Control and Prevention, Atlanta, Georgia United States of America (USA), ${ }^{4}$ Influenza Program, Centers for Disease Control and Prevention, Pretoria, South Africa, ${ }^{5}$ Medical Research Council: Respiratory and Meningeal Pathogens Research Unit, School of Pathology, University of the Witwatersrand, Johannesburg, South Africa, ${ }^{6}$ Division of Public Health Surveillance and Response, National Institute for Communicable Diseases of the National Health Laboratory Service, Johannesburg, South Africa, 'Department of Internal Medicine, Charlotte Maxeke Johannesburg Academic Hospital, Johannesburg, South Africa, ${ }^{8}$ University of the Witwatersrand, Johannesburg, South Africa, ${ }^{9}$ Department of Paediatrics, Steve Biko Academic Hospital, Pretoria, South Africa, ${ }^{10}$ Johns Hopkins Bloomberg School of Public Health, International Vaccine Access Center, Department of International Health, Baltimore, Maryland, USA, "' Hubert School of Public Health, Emory University, Atlanta, GA, USA

Streptococcus pneumoniae is a leading cause of severe bacterial infections globally. Pneumococcal disease burden data specific for South Africa are needed to better understand the impact of the pneumococcal conjugate vaccine (PCV) program following its introduction in 2009. We developed a model to estimate national burden of severe pneumococcal disease pre- (average 2005-2008) and post-PCV introduction (2013) in children aged $<5$ years in South Africa. We estimated case numbers using invasive pneumococcal disease incidence from national laboratory-based surveillance, adjusted for specimen-taking practices. Vaccine probe study data determined non-bacteraemic pneumonia incidence. To estimate pneumococcal deaths, we applied observed case fatality ratios to estimated cases. We conducted a sensitivity analysis to assess effect of different assumptions. Bootstrapping was used for confidence intervals. In the pre-vaccine era a total of 196,140 (178,490-210,370) cases of severe pneumococcal disease were estimated annually. In 2013, 67,270 cases were estimated; 128,870 cases averted over 5 years following PCV introduction, a rate difference of 2528 per 100,000 child-years. Approximately 8550 (6970-10220) pneumococcal-related deaths were estimated in the pre-vaccine period. In 2013, an estimated 5000 deaths were averted over the 5 year period following PCV introduction, a rate difference of 99 per 100,000 child-years. Incidence and mortality rates were higher in infants and HIV-infected children. In conjunction with other interventions, PCV had a significant impact on the high burden of pneumococcal hospitalisations and deaths in children aged $<5$ years in South Africa within 5 years of introduction.

Key words: Pneumococcal Disease, PCV, Children, Burden

\section{ID-O-4: TEMPORAL ASSOCIATION OF PNEUMOCOCCAL CONJUGATE VACCINE ON ALL-CAUSE PNEUMONIA HOSPITALIZATION AMONG CHILDREN IN SOWETO, SOUTH AFRICAN: A TIME SERIES ANALYSIS FROM 2006-2014}

Alane IzU', Fatima Solomon², Susan Nzenze ${ }^{2}$, Elizabeth Zel'3l, Katherine O'Brien4, Cynthia Whitney ${ }^{5}$, Jennifer Verani, Michelle Groome2, Shabir Madhi ${ }^{2,6}$

'School of Pathology, Respiratory \& Meningeal Pathogens Research Unit, University of the Witwatersrand, ${ }^{2}$ Medical Research Council: Respiratory \& Meningeal Pathogens Research Unit, University of the Witwatersrand, Department of Science and Technology/ National Research Foundation: Vaccine Preventable Diseases, ${ }^{3}$ Stat Epi Associates, Inc, ${ }^{4}$ International Vaccine Access Center, Department of International Health, Johns Hopkins Bloomberg School of Public Health, Baltimore, USA, ${ }^{5}$ Centers for Diseases Control and Prevention, Atlanta, USA, ${ }^{\circ}$ NeNational Institute for Communicable Diseases, National Health Laboratory Service, Sandringham, South Africa

In April 2009, a seven-valent pneumococcal conjugate vaccine (PCV7) was included in South Africa's routine immunization program; PCV13 replaced PCV7 in May 2011. We assessed PCV impact on all-cause pneumonia hospitalizations among Sowetan children. We examined pneumonia hospitalizations between 2006 and 2014 at Chis Hani Baragwanath Hospital in Soweto. We used prePCV data (2006-2008) in a Bayesian generalized seasonal autoregressive moving average model to estimate the number of all-cause pneumonia hospitalizations that would have occurred in the absence of PCV during 2010-2014 by HIV status and age group $(0-<3 \mathrm{~m}, 3-<12 \mathrm{~m}$, $1-<2 y$ and $2-<5 y$ ). To estimate PCV impact, we compared the observed and modelled number of pneumonia hospitalisations, reporting the median and $50 \%$ credible interval (Cl) of the posterior distribution. Overall 27,633 children were hospitalized for pneumonia between 2006 and 2014; 12.4\% were HIV-infected. Among HIVuninfected and HIV-unknown children $<5$ years, significant reductions in pneumonia hospitalization were observed by $2014(-44 \%, 50 \% \mathrm{Cl}-29$ to -57$)$. Declines were significant in $0-<3 \mathrm{~m}(-45 \% ; 50 \% \mathrm{Cl}:-10$ to -65$), 2-<5 y \quad(-41 \%$ : $50 \% \mathrm{Cl}-4$ to -62$)$, and $3-<12 \mathrm{~m}(-38 \%: 50 \% \mathrm{Cl}-9$ to -56$)$, but not $1-<2 y$ age group. Among HIV-infected children, pneumonia hospitalizations decreased but the estimated reduction 
was only significant in 2013 in $0-<3 m(-44 \%, 50 \% \mathrm{Cl}:-12$ to $-63)$ and $2-<5 y-(53 \%, 50 \% \mathrm{Cl}$ : 8 to -73$)$ age groups. These are the first data from Africa demonstrating PCV7/PCV 13 impact on pneumonia hospitalization trends among children. Hospitalizations dropped for HIV-infected children, but the decline preceded PCV introduction and likely reflects both expanding HIV-related programs and PCV immunization.

Key words: PCV, time series

\section{ID-0-5: MOLECULAR TYPING OF MULTIDRUG-RESISTANT CANDIDA AURIS STRAINS IN SOUTH AFRICA}

Rindidzani Magobo 1,2, Craig Corcoran ${ }^{3}$, Nelesh P. Govender ${ }^{2,4}$

'School of Pathology, Department of Clinical Microbiology and infectious Diseases, ${ }^{2}$ National Institute for Communicable diseases- Centre for Opportunistic, Tropical, hospital infections, ${ }^{3}$ Ampath National reference Laboratory, ${ }^{4}$ Faculty of Health Sciences, University of the Witwatersrand, Johannesburg, South Africa

Candida auris is an emerging, multidrug-resistant pathogen responsible for a wide spectrum of clinical manifestations and high mortality. We investigated the molecular relatedness among $C$. auris isolates circulating in South Africa. Eighty isolates collected from public and private sector hospitals in Gauteng province between 2012 and 2015 were analysed. Patient information including age, sex, specimen type and date of specimen collection was recorded. ITS and DI/D2 sequencing was done for species-level identification. Antifungal susceptibility testing (AST) against 9 antifungal drugs was done using Sensititre YeastOne method. CLSI clinical breakpoints for Candida albicans were used to interpret the AST results. FKS sequencing targeting hot spots 1 and 2 regions of FKS 1 gene was performed on all isolates showing phenotypic resistance to echinocandin. ITS, D1/D2, RPB1 and RPB2 regions of the ribosomal DNA gene were sequenced for multilocus sequence typing (MLST). Neighbour-joining phylogenetic tree was constructed using MLST data to identify clusters. Seventy nine isolates were identified as C. auris and 1 isolate as C. haemulonii. All isolates were resistant to fluconazole, $8 \%$ to voriconazole, $9 \%$ to echinocandins and $50 \%$ to amphotericin B. No FKS mutations were detected when comparing to wild-type $C$. auris strains. Two clusters were identified using MLST; cluster 1 and cluster 2 comprising of 77 and 2 isolates respectively. The median age was 59 years; males accounting for $61 \%$ of the population. Multidrug-resistant C. auris strains circulating in South African hospitals were highly related and there is a need to investigate possibility of nosocomial transmission using microsatellite genotyping.

Key words: Candida auris, multilocus sequence typing

\section{ID-0-6: GENETIC TARGETING OF THE HUMAN CCR5 GENE BY AN ENHANCED CRISPR/SACAS9 NICKASE SYSTEM}

Britt Hanson, Marco Weinberg

Faculty of Health Sciences, Department of Molecular Medicine and Haematology, HIV Pathogenesis Research Unit, University of the Witwatersrand

The human immunodeficiency virus (HIV) is a devastating and currently incurable disease. Antiretroviral (ARV) treatments have reduced the morbidity and mortality of the disease over the past decade and a half but none confer permanent immunity thus HIV will unlikely be eradicated in the near future. Proviral HIV genomes within infected host immune cells generate a latent viral reservoir, precluding full elimination of replicative viral particles. Gene editing is a promising tool for development of a therapeutic able to disrupt essential HIV or human elements permanently. The major HIV entry cell surface receptor, human CD4, is critical for normal immune functioning but the requisite co-receptors, human CCR5 or CXCR4, appear to be dispensable. The 'founder' virus in most sexually transmitted cases has an affinity for the CCR5 co-receptor (R5-tropic) and thus this was selected as the therapeutic target. A highly specific, or 'enhanced', and AAV-deliverable CRISPR/Cas9 nickase system derived from Staphylococcus aureus has been engineered to target and disrupt the human CCR5 gene within exon 3 , the only coding exon. Using a human T cell model, the mutation frequency of the system, effect on CCR5 cell surface expression and level of HIV-1 Subtype $C$ infection will be investigated further. Prevention of HIV-1 entry into the host immune cells responsible for the disease phenotype could be developed into a curative and prophylactic therapy for R5-tropic viral infection. This could greatly improve the cost of treatment as well as the livelihood of people globally and especially those most ravaged by the disease.

Key words: CRISPR/Cas9, CCR5, HIV, Gene Editing

\section{ID-0-7: CHARACTERIZATION OF BROADLY NEUTRALIZING ANTIBODY RESPONSES IN HIV-1 SUBTYPE C INFECTED CHILDREN}

Zanele Ditse ${ }^{1,2}$, Owen Karimanzira², Maximilian Muenchhoff ${ }^{3}$ Emily Adland4, Ivelin Georgiev', Philip Goulder ${ }^{3,5}$, Penny L. Moore², Lynn Morris

'School of Pathology, HIV Pathogenesis Research Unit, University of the Witwatersrand, ${ }^{2}$ Centre for HIV and STIs, National Institute for Communicable Diseases of the National Health Laboratory Service, Johannesburg, South Africa, ${ }^{3}$ Department of Paediatrics and HIV Pathogenesis Programme, University of Oxford, UK HIV Pathogenesis Programme, The Doris Duke Medical Research Institute, University of KwaZulu-Natal, Durban, South Africa, ${ }^{4}$ Department of Paediatrics and HIV Pathogenesis Programme, University of Oxford, UK, ${ }^{5}$ Department of Paediatric Infectious Diseases, Great Ormond St Hospital for Children, London, UK ${ }^{6} \mathrm{Vaccine}$ Research Centre, National Institute of Allergy and Infectious Diseases, National Institute of Health, Bethesda, USA 
Broadly neutralizing antibodies (bNAbs) have been isolated from multiple HIV-infected donors. These monoclonal antibodies (mAbs) have defined 5 targets on the HIV1 envelope: the CD4-binding site (CD4bs), glycan-V2, glycan-V3, membrane-proximal external region (MPER) and the gp120-gp41 interface epitopes. Only one study has examined bNAbs in children and showed that these arose earlier than in adults although the specificities could not be mapped. In this study we characterized bNAb responses in South African HIV-1 infected children. Plasma samples from 87 ART-naive children (median age of 7.9 years) were tested for neutralization breadth and potency using a panel of heterologous viruses in the TZMbl neutralizing antibody assay. The specificity of antibodies was mapped using epitope-ablating mutants and a bioinformatic analysis. Neutralization breadth in children was higher compared to an adult cohort matched for duration of infection. Furthermore, potency was higher with titres >1:1,000 being 4.8-fold more common in paediatric infection than in adults. Consistent with adults, viral load in children was associated with neutralization breadth. Computational and experimental mapping identified bNAbs targeting the MPER, CD4bs, gp120gp41 interface, glycan-V2, and glycan-V3 epitopes with the latter two specificities most common. Plasma from a mother-child pair showed similar neutralization profiles and titres, however, their specificities did not overlap completely. HIV-1 infected children develop broader and potent bNAb responses than adults and target known neutralization epitopes. Further studies on the mother-child pair will aid in understanding the role of viral determinants of neutralization breadth in paediatric HIV infection.

Key words: Paediatric HIV infection, broadly neutralizing antibodies, HIV-1 neutralization epitopes

\section{ID-O-8: GENETIC DETERMINANTS OF BROAD AND POTENT NEUTRALIZATION IN THE CAP256-VRC26 HIV-1 V1V2 SPECIFIC BNAB LINEAGE}

David Sacks ${ }^{1,2}$, Jinal Bhiman ${ }^{1,2}$, Lynn Morris ${ }^{1,2,3}$, Penny Moore

'School of Pathology, University of the Witwatersrand, NICD HIV \& STIS, ${ }^{2}$ Centre for HIV and STIS, National Institute for Communicable Diseases, National Health Laboratory Service, Johannesburg, South Africa, School of Pathology, University of the Witwatersrand, Johannesburg, South Africa, ${ }^{3}$ Centre for the AIDS Programme of Research in South Africa (CAPRISA), KwaZulu-Natal, South Africa

The CAP256-VRC26 lineage of V1V2-specific monoclonal antibodies (mAbs) consists of both broadly neutralizing (bNAbs) and narrowly neutralizing ("off-track") antibodies that have comparable levels of somatic hypermutation (SHM). We sought to elucidate the genetic determinants that functionally differentiate broad and narrow mAbs that cluster closely on a phylogenetic tree. Overlapping $\mathrm{PCR}$ was used to exchange the $\mathrm{CDRH} 3$ between
CAP256.27 (59\% breadth), and CAP256.20 (2\% breadth), both of which have $16 \%$ SHM. MAbs transfected into 293F cells were purified using Protein A and tested in an Envpseudotyped neutralization assay against $17 \mathrm{HIV}-1$ clade $A, B$ and $C$ viruses. Introduction of the bNAb CAP256.27 $\mathrm{CDRH} 3$ into the narrow mAb CAP256.20 altered 6 amino acids causing a net $\mathrm{CDRH} 3$ charge change from 0 to -4. This introduced neutralization against 13 of 14 viruses, with the chimera exhibiting equivalent potency to the CAP256.27 bNAb. Conversely insertion of the CDRH3 from CAP256.20 into the bNAb ablated neutralization of all viruses. Two mutations mutations ( $\mathrm{W} 100 \mathrm{~d}$ and $\mathrm{H} 100 \mathrm{k}$ ) were unique to CAP256.20 and predicted by modeling (Gorman et al, 2016) to influence the breadth by displacing the side chain of $\mathrm{K} 168$ and disrupting contact with $\mathrm{K} 169$ in the V2 epitope. A restricted number of residues within the $\mathrm{CDRH} 3$ confers strain-specificity in CAP256.20, likely by reducing polar interactions with key residues in the viral epitope. These data define paratope contact sites that differ between narrow and broad antibodies, and inform the design of immunogens that minimize off-track antibody elicitation.

Key words: HIV, broadly, neutralizing, antibody

\section{ID-0-9: CCL3L AND CCL4L GENE COPY NUMBERS AND CONTROL OF HIV-1 INFECTION}

Sizanani Mncube ${ }^{1,2}$, Maria Paximadis ${ }^{1,2}$, Ziyaad Waja ${ }^{3,4}$, Neil Martinson $^{3,4}$, Caroline T Tiemessen ${ }^{1,2,4}$

'Department of Virology, School of Pathology, Faculty of Health Sciences, University of the Witwatersrand, Johannesburg, South Africa, ${ }^{2}$ Centre for HIV and STIs, National Institute for Communicable Diseases, National Health Laboratory Service, Johannesburg, South Africa, ${ }^{3}$ Perinatal HIV Research Unit, Faculty of Health Sciences, University of the Witwatersrand, Johannesburg, South Africa, ${ }^{4}$ Medical Research Council Soweto Matlosana Centre for HIV/AIDS and TB Research, Johannesburg, South Africa

Two chemokine ligands that bind the CCR5 receptor, namely CCL3 and CCL4, are encoded by CCL3/CCL3L and the CCL4/CCL4L genes, respectively. CCL3L and CCL4L copy numbers lower than the population median have been associated with increased susceptibility to HIV-1 infection and faster rate of disease progression. In this study we assessed the role of CCL3L (CCL3La and CCL3Lb) and CCL4L (CCL4La and CCL4Lb) gene copy numbers in natural control of HIV-1 infection in the Black South African population. Droplet digital PCR was used to determine gene copy numbers for HIV-1-infected controllers (HICs, $n=52$ ), divided into Elite Controllers [ECs, $n=11$, viral load (VL) $<50$ RNA copies/ml, without antiretrovirals (ARVs)], Viraemic Controllers (VCs; $n=30$, $\mathrm{VL}<2000$ RNA copies/ml, without ARVs) and High Viral Load Long Term Non-progressors (HVL LTNPs; $n=11, V L>$ 10000 RNA copies/ml, CD4 $>500$ cells/ $\mu$ for $>7$ years 
without ARVs) and HIV-1-infected progressors ( $n=74, C D 4$ $<350$ cells/ $\mu$ l, requiring ARV treatment). We found CCL3La copy number to be equal to CCL4L copy number in all individuals with the exception of one individual. Significant differences were seen when comparing CCL3La/CCL4L copy numbers between HICs and progressors ( $p=0.045)$, $V C s$ and progressors $(p=0.031)$ and also when comparing HICs with $\mathrm{VL}<400$ RNA copies/ml ( $<400 \mathrm{HICs}$ ) to progressors $(p=0.01)$, with the controllers having higher representation of individuals with copy numbers greater or equal to the study population median. These findings suggest that these two chemokines are playing a role in natural control of HIV-1.

Key words: HIV-1, CCL3, CCL4, Copy number

\section{ID-0-10: P21 EXPRESSION AND HIV-1 CONTROL IN A BLACK SOUTH AFRICAN POPULATION}

Gemma Whitney Koor ${ }^{1,2}$, Maria Paximadis ${ }^{1,2}$, Ziyaad Waja ${ }^{3,4}$, Neil Martinson $^{3}, 4$, Caroline T Tiemessen ${ }^{1,2,4}$

'School of Pathology, Department of Virology, University of the Witwatersrand, ${ }^{2}$ Centre for HIV \& STIs, National Institute for Communicable Diseases, National Health Laboratory Service, Johannesburg, South Africa, ${ }^{3}$ Perinatal HIV Research Unit, Faculty of Health Sciences, University of the Witwatersrand, Johannesburg, South Africa, ${ }^{4}$ Medical Research Council Soweto Matlosana Centre for HIV/AIDS and TB Research, Johannesburg, South Africa

HIV-1 infected individuals show variation in rates of disease progression. p21, a cyclin dependent kinase inhibitor, has been implicated in HIV-1 control, with elite controllers (ECs; $>450 \mathrm{CD} 4+\mathrm{T}$ cells/ $\mu \mathrm{l}$, viral load (VL) <50 RNA copies/ml without the use of antiretrovirals) and viraemic controllers (VCs; >450 CD4+ T cells/ $\mu$ l, VL <2000 RNA copies/ml without the use of antiretrovirals) exhibiting higher p21 expression in CD4+ T cells compared to progressors as well as to HIV-1 negative individuals. We measured levels of p21 mRNA, a marker for p21 expression, in purified CD4+ T cells of Black South African healthy controls $(n=10)$, ECs $(n=11)$, VCs $(n=10)$, high VL long term non-progressors ( $n=4 ;>500$ CD4+ T cells/ $\mu$ for $>7$ years, $V L>10,000$ RNA copies/ml) and progressors ( $n=12 ;<250$ CD4+ T cells/ $\mu \mathrm{l}, \mathrm{VL}>10,000$ RNA copies/ml). An assay was developed to genotype a 5'UTR p21 SNP, rs733590, that alone and together with $\mathrm{HLA}-B^{*} 2705$, has been shown to impact p21 expression. p21 mRNA expression significantly correlated with markers of disease progression (VL: $r=0.69, p<0.0001$; CD4+ T cell count: $r=-0.53, p=0.0005)$. ECs and VCs had significantly lower p21 mRNA expression when compared to progressors $(p=0.002$ and $p=0.001$, respectively). The p21 5'UTR rs733590 SNP had no impact on p21 mRNA expression in our study population. These findings, together with the absence of HLA-B*2705 in Black South Africans, point to host genetic differences as the likely contributors to the different results seen in our study.

Key words: p21, HIV-1, Natural Control, Elite Controllers

\section{ID-0-11: PERSISTENCE OF TRANSMITTED ANTIRETROVIRAL DRUG RESISTANCE MUTATIONS IN THE IAVI-EARLY HIV INFECTION COHORT (PROTOCOL C)}

Dean Harris', Maria Papathanasopoulos², Simon Travers³, Matt Price $^{4}$

'School of Pathology, Department of Molecular Medicine and Haematology, HIV Pathogenesis Research Unit, ${ }^{2}$ University of the Witwatersrand, 3 South African National Bioinformatics Institute, ${ }^{4}$ International AIDS Vaccinelnitiative There is a paucity of data for transmitted antiretroviral drug resistant (TDR) HIV-1. The 29 individuals identified with TDR from the IAVI-Early HIV Infection Cohort (Protocol C) provides an opportunity to address this issue. This study characterized evolutionary mechanisms of antiretroviral drug resistant HIV-l after transmission, and provided insights into persistence and/or rates of reversion. Viral RNA was extracted from longitudinal plasma samples $(n=216)$ from 29 individuals with early HIV-1 infection from Kenya, Uganda, Rwanda, Zambia and South Africa with baseline TDR mutations. RT-PCR amplified and sequenced on the Illumina Miseq. ARV drug resistance mutations on the CPR and Stanford HIV Drug Resistance Database were identified with the Exatype software (1\%cut-off).Data is currently available for 15 individuals. TDRMs identified by Sanger and MiSeq sequencing correlated and additional minority variants were detected by MiSeq. TDRMs included K103N (n=7), M46L (n=1), I85V (n=1), Y188C (n=1), K103N and Y181C (n=1), K103E and E138A $(n=1), A 98 G, E 138 Q$, Y181C and M184V (n=1), K103N and E138A (n=1) and K103N, V108I and M184V $(n=1)$. The TDRMs identified in 13 out of 15 individuals persisted in varying proportions for the follow up periods. Sequenced virus from patient 10 showed reversion of $\mathrm{K} 103 \mathrm{E}(64 \%$ to $<1 \%)$ and $\mathrm{E} 138 \mathrm{~A}(75 \%$ to $<1 \%$ ) within three months, but G190A detected at baseline $(24 \%)$ emerged as the predominant quasispecies by month $3(99 \%)$, and persisted throughout. Virus from patient 20 showed persistence of $\mathrm{K} 103 \mathrm{~N}$ but reversion of $\mathrm{V} 108 \mathrm{l}$ and M184V.TDRMs appear to persist after transmission to a new host. Results highlight that understanding population dynamics of TDR HIV-1 in the absence of drug pressure is essential for clinical management, public health strategies and informing future vaccine design.

Key words: HIV, Drug, Resistance, Sequencing

\section{ID-O-12: UPTAKE AND CLINICAL OUTCOMES FROM A PRIMARY HEALTHCARE BASED SAFER CONCEPTION SERVICE IN JOHANNESBURG, SOUTH AFRICA: FINDINGS AT 7 MONTHS}

Natasha Davies ${ }^{1,2}$, Saiqa Mullick2, Sheree Schwartz ${ }^{2,3}$

'School of Clinical Medicine, Faculty of Health Sciences, ${ }^{2}$ Wits Reproductive Health and HIV Institute (WRHI), 3JHU

Safer-conception services (SCS) for HIV-affected couples support HIV counselling and testing (HCT), ART 
initiation, viral load (VL) suppression and HIV prevention. We implemented a primary healthcare-based SCS in Johannesburg. We report early uptake and clinical outcomes. HIV-affected individuals/couples desiring pregnancy enrolled from existing PHC services. Saferconception strategies offered included: HCT, ART initiation, VL monitoring, STI screening/management, and periovulatory condomless sex or self-insemination. Of 218 participants, 141 (65\%) are female (average 33.5years; range 19-45) and 77 male (average 37.4years, range 24-57). Overall, 134/141 (95\%) women and 62/77 (81\%) men are HIV-positive, including five newly diagnosed. No seroconversions have occurred amongst $22 \mathrm{HIV}$-negative clients. 51 seroconcordant and 23 serodiscordant couples enrolled, plus 70 unaccompanied individuals of whom 27 report seroconcordant relationships, 16 serodiscordant and 27 unknown partner status. Tailored safer-conception strategies were offered based on initial assessment. Twenty-one ARV-naïve clients initiated treatment. 9/11 on ART for $\geq 3$ months had $V L<200$ copies. 9/172 (5\%) ARTexperienced clients had $V L>200$ copies. All received adherence support, four required switch to second-line therapy. Fourteen pregnancies have been confirmed, two via self-insemination. Eight (57\%) pregnancies followed optimal SC strategy use, four attended only one consultation, and two partners were not yet on ART. Thirteen (93\%) of the women were virally suppressed at pregnancy confirmation. All attended antenatal care (ANC) $\leq 7$ weeks' gestation. Implementing a PHC-based SCS is acceptable and feasible, impacting HCT, ART initiations, VL suppression and PMTCT, including earlier ANC booking. Challenges include partner non-disclosure, difficulties engaging men and pregnancies occurring without optimal SC strategies.

Key words: HIV safer conception implementation

\section{ID-O-13: INITIATING ART AT A PATIENT'S FIRST CLINIC VISIT: THE RAPIT RANDOMIZED TRIAL}

Lawrence Long', Sydney Rosen², Mhairi Maskew3 ${ }^{3}$, Matt Fox ${ }^{2}$, Cynthia Nyoni, Constance Mongwenayana ${ }^{3}$, Given Malete ${ }^{3}$, lan Sanne 3

'School of Clinical Medicine, Faculty of Health Sciences, University of the Witwatersrand, Health Economics and Epidemiology Research Office, ${ }^{2}$ Boston University, ${ }^{3}$ University of Witwatersrand

High rates of patient attrition from HIV care between HIV testing and ART initiation have been documented in subSaharan Africa. We estimated the effect of accelerated initiation procedures on uptake of ART and viral suppression. We conducted a randomized controlled trial of same-day ART initiation in two public sector clinics in South Africa. Adult ( $\geq 18)$, non-pregnant patients receiving a positive HIV test or first CD4 count were randomized to standard or rapid initiation. On enrollment, rapid arm patients received point-of-care laboratory tests and accelerated initiation procedures to allow immediate dispensing of ARVs Standard arm patients followed standard clinic procedures for ART initiation. Follow up was by medical record review. Of 600 patients screened, 377 were eligible for ART and enrollment (56\% female, median age 35 , median CD4 count 210). In the rapid arm 182/187 initiated ART $\leq 90$ days, compared to 136/190 in the standard arm (RR 1.36; 1.24-1.49). In the rapid arm, 119/187 initiated and were suppressed at 10-months, compared to $96 / 190$ in the standard arm (RR $1.26 ; 1.05-1.50) .72 \%$ of rapid arm patients were initiated on the same day as study enrollment. A larger effect was observed at the primary health clinic site, compared to the hospital-based clinic, and among younger men. Offering same-day ART initiation to adult patients in South Africa increased uptake of ART by $36 \%$ and viral suppression by $26 \%$. It is feasible and acceptable in public sector clinics and may be possible with fewer POC instruments.

Key words: HIV, POC, ART, Attrition

\section{ID-O-14: BIOGRAPHIES OF HIV AND CERVICAL CANCER: UNDERSTANDING TREATMENT-SEEKING AMONGST HIV POSITIVE WOMEN IN INNER CITY JOHANNESBURG}

Jonathan Stadler', Admire Chikandiwa', Philippe Mayaud², Helen Rees', John Inrie', Sinead Delany-Moretlwe'

'Wits Reproductive Health and HIV Institute (WRHI), '2London School of Hygiene and Tropical Medicine

In the 'HPV in Africa Research Partnership' (HARP) study over a quarter $(23 / 129,26 \%)$ of HIV positive women diagnosed with CIN2 did not access surgical treatment. Our qualitative research explored why. A purposive subsample ( $N=30$ ) was selected for in depth interviews (IDI), of which 15 had received surgical treatment for CIN2 and 15 not. Of these, five of each were invited to a second interview. The McGill Illness Narrative Interview tool (www. mcgill.ca) was used to elicit: (1) a chronology of symptoms and illness experiences; (2) popular representations of illness; and (3) explanatory models of illness and treatment. IDI were conducted in local languages, transcribed and translated and coded in Nvivo 10. Twenty study participants ( 16 treated and 4 untreated) attended one IDI and nine participated in a second. Twelve were on ARV and 16 were treated surgically for CIN2. IIIness narratives reflected shared experiences of intimate partner violence (IPV), domestic instability, and material deprivation. These experiences shaped a collective perception of cancer as an untreatable and hopeless illness that threatened productive and reproductive futures. In contrast, HIV was well understood and regarded as manageable. Access to cash, and supportive household relationships facilitated women's access to treatment for HIV and CIN2. However, all women experienced delays due to difficulties negotiating health care services. Health services need to address women's perceptions of cancer, but also remove 
barriers to immediate treatment. Screen and treat options and a HPV vaccine for HIV positive women are important interventions to prevent cervical cancer.

Key words: HIV, HPV, Cervical Cancer, Biographies

\section{ID-O-15: ANOGENITAL HPV INFECTION AND ASSOCIATED DISEASE IN HIV- POSITIVE MEN IN JOHANNESBURG, SOUTH AFRICA.}

Admire Chikandiwa', Lucy Chimoyi', Pedro.T. Pisa', Pamela Michelow ${ }^{2}$, Philippe Mayaud ${ }^{3}$, Sinead Delany-Moretlwe

'School of Clinical Medicine, Wits Reproductive Health and HIV Institute (WRHI), 'Cytology Unit, National Health Laboratory Service and Dept of Anatomical Pathology, ${ }^{3}$ London School of Hygiene and Tropical Medicine

High-risk human papillomavirus (HR-HPV) infection is associated with anogenital and oropharyngeal cancers, particularly in men living with HIV/AIDS (MLWHA). We enrolled 304 sexually active consenting MLWHA $\geq 18$ years. Socio-behavioral data was collected by questionnaire. Clinicians examined for anogenital warts (AGWs), collected blood (CD4+ counts, HIV viral load [VL]), genital ( $n=283$ ), anal ( $n=227$ ) swabs for HPV DNA (Roche Linear Array) and anal smears. Factors associated with anogenital disease were assessed by regression. The median age was 38 (IQR 10) years. Most (65\%) participants were on ART, $20 \%$ of those on ART for $>12$ months had undetectable $\mathrm{VL}$. Among those with anogenital swab results, $79 \%$ had HPV detectable, 52\% had HR-HPV and $64 \%$ had LR-HPV. The prevalence of AGWs was 12\%. Infection with HPV 6 [aOR=3.79 (1.4-9.9), $\geq 1$ LR types [aOR=42.67 (14.06-129.48)] and being $\leq 6$ months on ART [aOR=6.56 (1.66-22.97)] were associated with AGWs. VL was not associated with AGWs. Overall, $39 \%$ of anal swabs had detectable HPV, $25 \%$ had any HR-HPV, $7 \%$ had $>1$ HR infection, $22 \%$ had any LR-HPV. Of the 242 anal smears with valid results, $51 \%$ were normal, whilst $20 \%$ and $29 \%$ were ASCUS and LSIL respectively. Infection with $\geq 1 \mathrm{HR}$ types $[\mathrm{aOR}=1.71$ (1.04-2.81)] was associated with abnormal cytology. VL was not associated with abnormal cytology. AGWs and cytological abnormalities were prevalent. Infection with $\geq 1$ type was associated with disease. Further research is needed to understand progression of infection to disease and inform prevention strategies.

Key words: Anogenital, HPV, HIV, Men

\section{ID-0-16: COMPOSITE MARKER TO IDENTIFY POOR ADHERENCE AMONG ART NAÏVE PATIENTS ON FIRST-LINE REGIMEN AT THEMBA LETHU CLINIC IN JOHANNESBURG: RESULTS FROM A COHORT STUDY}

Mouhamed Mbenguel, Charles Chasela', Matthew P Fox ${ }^{3}$, Denise Evans ${ }^{4}$
ISchool of Public Health, Department of Epidemiology and Biostatistics, Wits Reproductive Health and HIV Institute (WRHI),

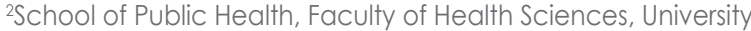
of the Witwatersrand, ${ }^{3}$ Department of Global Health, Boston University School of Public Health, Boston, MA, USA, ${ }^{4}$ Health Economics and Epidemiology Research Office, Department of Internal Medicine, School of Clinical Medicine, Faculty of Health Sciences, University of the Witwatersrand

Viral load is the gold standard for measuring adherence to ART. However, this technique is not routinely available. We examined prospectively the diagnostic value of a composite marker to identify poor ART adherence. Consecutive HIV-positive patients initiating first-line ART were enrolled between 02/2012 and 05/2014 at Themba Lethu Clinic, Johannesburg. At the 6 month's follow-up, adherence was assessed using the selfreported adherence (SRA) tools: a self-assessment, pill identification test (PIT), visual analog scale (VAS) and Simplified Medication Adherence Questionnaire (SMAQ). The primary outcome was viral load $\geq 400$ copies $/ \mathrm{ml}$. We tested the association between viral load, baseline characteristics, biomarkers changes, number of missed visits and SRA measures. Adjusted relative risks were rounded and added together to obtain a risk score. We determined sensitivity (Se), specificity (Sp), positive predictive value (PPV) and negative predictive value (NPV) at different thresholds compared to viral load. 296 patients were included. Factors associated with viral load $\geq 400$ copies $/ \mathrm{ml}$ were male gender, platelet count $<150$ cells/mm3, VAS score $<95 \%$, missing at least two ARV visits by $\geq 7$ days and change in MCV <14.5fL. The ROC area of the risk score was 0.61 . The optimal diagnostic accuracy was obtained with the score of 5 ( $\geq 5$ vs $<5$; Se $64 \%$ Sp 50\%). Se and Sp of SRA methods were: VAS $24 \%$ and $87 \%$, SMAQ $26 \%$ and $79 \%$, combination of self-assessment methods: $18 \%, 86 \%$. Composite marker based on routine data should be preferred over self-reported adherence methods in identifying patients with poor ART adherence.

Key words: HIV, antiretroviral, adherence, viral load

\section{ID-0-17: THE BURDEN AND CLINICAL PRESENTATION OF PULMONARY TUBERCULOSIS IN ADULTS WITH SEVERE RESPIRATORY ILLNESS IN A HIGH HIV PREVALENCE SETTING, 2012-2014}

Sibongile Walaza', Andries Dreyer', Halima Dowood ${ }^{3}$, Ebrahim Variava $^{4}$, Neil Martinson ${ }^{5}$, Jocelyn Moyes², Nicole Wolter ${ }^{2}$, Nazir Ismail2, Cheryl Cohen ${ }^{2}$

'School of Public Health, ${ }^{2}$ National Institute for Communicable Diseases, ${ }^{3}$ Pietermaritzburg Metropolitan Hospital Complex, Klerksdorp Tshepong Hospital Complex, ${ }^{4}$ Perinatal HIV Research Unit

Understanding the burden and clinical presentation of tuberculosis in patients with severe respiratory illness (SRI) has important implications for anticipating treatment requirements and designing appropriate public health 
strategies. Hospitalized patients aged $\geq 15$ years with SRI were enrolled prospectively in two provinces of South Africa from 2012- 2014. Tuberculosis testing included smear microscopy, culture or Xpert MTB/Rif. Blood was tested for pneumococcus by real-time PCR. We enrolled 2,486 individuals with SRI. Of these, 2,097 (84\%) patients were tested for tuberculosis, 593 (28\%) were positive. HIV prevalence was 75\% (1014/1355) and 81\% (424/522) in tuberculosis negative and positive cases respectively. Tuberculosis detection rate was $18 \%$ (133/729) in individuals with acute $(\leq 14$ days) and $34 \%(460 / 1,368)$ in those with chronic (>14 days) presentation. Among cases with laboratory-confirmed tuberculosis, those with acute presentation were less likely to present with cough [ $88 \%$ (117/133) vs 97\% (447/460); aOR 0.2, 95\% Cl 0.1-0.5)], night sweats [57\% (75/132) vs $73 \%$ (337/459); aOR $0.4,95 \% \mathrm{Cl}$ 0.3-0.7)] or be started on tuberculosis treatment at current admission [63\% (78/124) vs $81 \%$ (344/423); aOR 0.4, 95\% $\mathrm{Cl}$ 0.3-0.7)] but were more likely to be co-infected with pneumococcus [13\% (16/124) vs 6\% (26/411); aOR 2.3, $95 \% \mathrm{Cl}$ 1.3-5.3)] than patients with chronic presentation. Annual incidence of acute and chronic tuberculosisassociated SRI per 100,000 population was 28 (22-39) and 116 (104-128) respectively. In this setting, tuberculosis is a common finding in patients hospitalized with SRI, including in patients with acute presentation who may be missed by tuberculosis screening guidelines.

Key words: Tuberculosis, Presentation, HIV

\section{ID-P-1: CELL-MEDIATED IMMUNE RESPONSES TO INACTIVATED INFLUENZA VACCINE IN HIV INFECTED AND HIV- UNINFECTED PREGNANT WOMEN}

Alexander Malherbe', Clare L. Cutland², Nadia van Niekerk², Shabir A. Madhi', ${ }^{2,3}$ Marta C. Nunes ${ }^{2}$

'School of Pathology, Faculty of Health Sciences, Respiratory and Meningeal Pathogens Research Unit, ${ }^{2}$ Medical Research Council: Respiratory and Meningeal Pathogens Research Unit, University of the Witwatersrand, Johannesburg, South Africa, Department of Science and Technology/National Research Foundation: Vaccine Preventable Diseases Unit, University of the Witwatersrand, Johannesburg, South Africa, ${ }^{3}$ National Institute of Communicable Diseases, National Health Laboratory Service, Sandringham, South Africa

Limited data exist on cell-mediated immune (CMI) responses to influenza vaccination in pregnant woman. We studied CMl responses to trivalent inactivated influenza vaccine (IIV3) in pregnant women with and without HIV-infection and compared these with non-pregnant women. A prospective study involving $263 \mathrm{HIV}$-infected pregnant (HIV+Preg), 105 HIV-infected non-pregnant (HIV+NP), 75 HIV-uninfected pregnant (HIV-Preg) and 75 HIV-uninfected non-pregnant (HIV-NP) women was undertaken in Soweto in 2013. Each participant received a single dose IIV3 and peripheral blood mononuclear cells
(PBMC) were isolated pre- and 28 days post-vaccination. $\mathrm{CMl}$ responses to the influenza vaccine strains were measured by interferon-y enzyme-linked immunospot (ELISPOT) assay. Positive responses were defined as $\geq 2$-fold increase in spot forming cells (SFU)/106 PBMCs, preto post-IIV3 and 10 SFU/106 PBMCs as the minimum value of detection. Participants who achieved $\geq 2$-fold increase in SFC/106 PBMCs post-IIV3 was similar among study groups, ranging from $16.3 \%-34.1 \%$ for $\mathrm{A} / \mathrm{H} 1 \mathrm{~N} 1 \mathrm{pdm}, 22.0 \%$ - $30.8 \%$ for $\mathrm{A} / \mathrm{H} 3 \mathrm{~N} 2$ and $17.4 \%-26.1 \%$ for B-Yamagata. Comparing pre- to post-IIV3, geometric mean SFU/106 PBMCs significantly increased for $\mathrm{A} / \mathrm{H} 1 \mathrm{~N} 1 \mathrm{pdm}$ in $\mathrm{HIV}+$ Preg $(p=0.0309)$ and HIV+NP ( $p=0.0094)$, respectively, and for $B-Y a m a g a t a$ in $\mathrm{HIV}+\mathrm{NP}(\mathrm{p}=0.0303)$. Low baseline SFU/106 PBMCs $(<60)$ were associated with better response to vaccination in $\mathrm{HIV}+$ Preg for $\mathrm{A} / \mathrm{HINIpdm}(\mathrm{p}=0.0002)$, $A / H 3 N 2(p=0.0008)$ and $B-Y a m a g a t a(p=0.0248), H I V+N P$ for $A / H 1 N 1 p d m(p=0.001)$ and B-Yamagata $(p=0.0145)$, and HIV-Preg for H3N2 ( $p=0.036)$. CMI responses to IIV3 were comparable among the study groups. Women with higher baseline responses had weaker boost of strainspecific T-cell immunity.

Key words: Cell-mediated immunity, HIV-infected pregnant women, Influenza vaccine

\section{ID-P-2: THE DECLINE INNON-CULTURABLE TUBERCLEBACTERIA IN HIV-POSITIVE AND HIV-NEGATIVE TUBERCULOSIS PATIENTS DURING EARLY FIRST-LINE TREATMENT}

Amanda Mclvor', Julian Peters', Andrea Papadopolous', Bhavna Gordhan', Neil Martinson", Matebogo Letutu, Bavesh Kana ${ }^{2}$

'School of Pathology, Faculty of Health Sciences, University of the Witwatersrand, Centre of Excellence for Biomedical TB Research, ${ }^{2}$ Perinatal HIV Research Unit, University of the Witwatersrand

Previous data have demonstrated the existence of differencially culturable tubercle bacilli (DCTB) in sputum samples from patients with active TB at baseline. These organisms do not form colonies on plates and their growth in liquid media is dependent on the addition of culture filtrate with or without resuscitation promoting factors (Rpfs), a group of bacterial growth stimulatory molecules. We aim to detect and quantify the relative proportions of DCTB and culturable bacteria at baseline and at various time points during the 'intensive phase' of first-line tuberculosis treatmentin both HIV positive and negative individuals. Most probable number (MPN) assays in liquid media and colony forming units (CFUs) on solid agar were used to determine the proportion of DCTB and culturable bacteria, respectively. Sixty patients consisting of $40 \mathrm{HIV}$ positive and $20 \mathrm{HIV}$ negative individuals were analysed. All patients harboured populations of DCTB that were isolated in culture filtrate with or without Rpfs suggesting factors other than Rpfs are required for growth. Our results indicate that HIV negative individuals harbor more DCTB 
than HIV positive individuals at baseline and during the first week of anti-TB treatmentas detected by the culture filtrate supplemented MPN assay $(p>0.05)$. Analysis of the rates of decline demonstrates an initial tolerance of DCTB to anti-TB treatment when compared to culturable populations and confirmed that protracted treatment is necessary to eliminate these organisms. Furthermore, our data confirm a novel role for DCTB in the transmission of diseaseby HIV negative individuals within the community and requires further investigation.

Key words: Tuberculosis, Dormancy, Resuscitation

\section{ID-P-3: THE ROLE OF PEPTIDOGLYCAN HYDROLASE ACTIVATORS IN REGULATING CELL DIVISION AND GROWTH OF MYCOBACTERIUM TUBERCULOSIS.}

Andreas Papadopoulos, Bavesh Kana

School of Pathology, University of the Witwatersrand, DST/NRF Centre of Excellence for Biomedical TB Research

The growing incidence of drug resistant strains of Mycobacterium tuberculosis and prevalent co-infection with HIV indicate the urgent need for alternative strategies to treat Tuberculosis. Targeting the process of bacterial cell division is a common approach towards drug design. Cell division in Escherichia coli is facilitated through the activity of peptidoglycan hydrolases, known as amidases. Amidases are produced in an inactive form and activation requires interaction with specific M23-domain proteins to allow for daughter cell separation. The aim of this research is to determine if M23-domain-containing proteins serve as activators of $M$. tuberculosis amidases and to ascertain a role of another peptidoglycan hydrolase, RpfB, in cell division by studying deletions of the M23-domain genes and rpfB in M. tuberculosis H37Rv. The resulting cell morphology will be compared to previouslycharacterised amidase-deficient strains, together with M23-domain gene expression analyses relative to bacterial growth phases and determining localisation of fluorescence-tagged M23-domain derivatives to the site of cell division. Thus far, bioinformatics analyses suggest the presence of 3 M23-domain genes in the M. tuberculosis genome. Two of these genes, designated Rv3786C and Rv2891, form a phylogenetic cluster with the E. coli activators, sharing important architectural elements including a degenerate M23-endopeptidase domain and signal peptide. The third and most conserved, Rv0950c, may function differentially, having a predicted active M23-endopeptidase domain. Characterisation of the M23-domain proteins is an important step in mapping the mechanisms of mycobacterial cell division and growth, offering strong potential for translation of this research into identification of novel drug targets.

Key words: TB, cell division, amidase, lytM

\section{ID-P-4: SUPPRESSION OF HEPATITIS B VIRUS REPLICATION USING MAL- PEGYLATED HELPER-DEPENDENT ADENOVIRAL VECTORS EXPRESSING PRI-MIRNAS}

Anele Mdunyelwa, Betty Maepa, Patrick Arbuthnot

School of Pathology, Department of Molecular Medicine and Haematology, Antiviral Gene Therapy Research Unit, University of the Witwatersrand, 7 York Road, Parkłown, South Africa

Hepatitis B virus (HBV) is a pathogen that causes acute hepatitis, chronic hepatitis or hepatocellular carcinoma by infecting the liver. Current HBV therapeutics are able to inhibit HBV replication, but they result in viral resistance and toxicity. To overcome these obstacles, Helper-Dependent Adenoviral vectors(HD Ads) expressing anti-HBV primarymicro RNAs (pri-miRNAs) from a liver-specific Mouse Transthyretin Receptor (MTTR) promoter were previously produced. However, as a result of immune stimulation by capsid proteins and $\beta$-galactosidase encoded by the vector used, short-term HBV inhibition was observed. To prolong HBV inhibition, lac $Z$ deficient and polyethylene glycol modified HD Ads expressing anti-HBV pri-miRNAs from MTTR promoter are used in this study. Adenoviral plasmids carrying anti-HBV sequences or luciferase were constructed and characterised in this study. Both anti-HBV sequence and luciferase expressing $\mathrm{HD}$ Ads are being produced in tissue culture. In vivo and in vitro anti-HBV sequence expression, silencing of HBV replication and the safety HD Ads used will be assessed. This study represents a useful model for the assessment of long term HBV repression with less immunogenicity by anti-HBV expression cassettes, thus leading to the development of drugs which may be used to treat chronic HBV with reduced side effects.

Key words: HBV, HD Ads, MTTR, pri-miRNA

\section{ID-P-5: COMPARING MALARIA VECTOR SURVEILLANCE TECHNIQUES IN MPUMALANGA PROVINCE, SOUTH AFRICA}

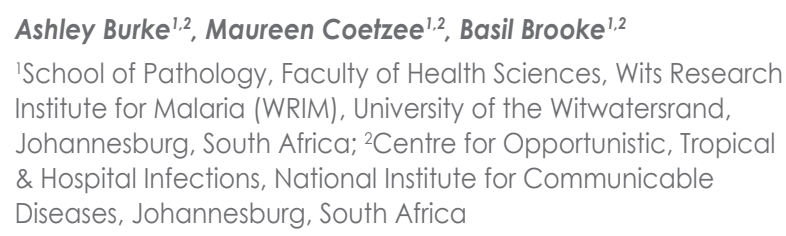
Institute for Malaria (WRIM), University of the Witwatersrand, Johannesburg, South Africa; ${ }^{2}$ Centre for Opportunistic, Tropical \& Hospital Infections, National Institute for Communicable Diseases, Johannesburg, South Africa

Insecticide based malaria vector control in South Africa has proven effective, but insecticide resistance has been recorded in populations of the primary vectors, which threatens control of malaria in high-risk areas. Thorough vector surveillance is required to understand the behaviour of local vector populations to enhance vector control. These studies aimed to asses and compare the efficacies of active and passive mosquito collection techniques and to 
establish the contribution of outdoor biting vectors to local malaria transmission. Anopheles mosquito surveillance was conducted for one year in two villages in Mpumalanga which experience seasonal malaria transmission. Ceramic pots and modified buckets were placed both inside and outside 20 randomly selected households as passive traps. Anopheline mosquitoes cleared from these traps were preserved and later identified to species, and all females were screened for Plasmodium falciparum sporozoites by ELISA. Active trapping took place every month during the malaria transmission season where humanlanding collections and $\mathrm{CO} 2$ baited nets were used. Both active trapping techniques were equally effective in collecting Anopheles mosquitoes, but were mostly of the An. gambiae complex. The outdoor traps proved the most efficient passive collection method, while few samples were collected indoors, which can be attributed to IRS operations in both villages. All female specimens tested negative for Plasmodium sporozoites, except for one An. vaneedeni female which tested positive through two ELISA assays. Molecular work is currently underway to confirm this Plasmodium positive specimen using a nested PCR assay. This is the first time that An. vaneedeni has been tentatively implicated in malaria transmission. The outdoor resting trait of this species will likely have important implications for the efficacy of IRS based malaria vector control in South Africa.

Key words: Malaria, Vector, Surveillance

\section{ID-P-6: MORTALITY IN CHILDREN <5 YEARS WITH SEVERE ACUTE RESPIRATORY ILLNESS IN URBAN AND RURAL AREAS, SOUTH AFRICA, 2009-2013.}

Oluwatosin Ayeni', Sibongile Walaza', Stefano Tempia ${ }^{3,4}$, Michelle Groome ${ }^{5,6}$, Kathleen Kahn ${ }^{7,8,9}$, Shabir A. Madhi' ${ }^{2,5,6}$ Cheryl Cohen ${ }^{1,2}$

'School of Public Health, Faculty of Health Sciences, University of the Witwatersrand, Johannesburg, South Africa, ${ }^{2}$ Centre for Respiratory Diseases and Meningitis, National Institute for Communicable Diseases of the National Health Laboratory Service, Johannesburg, South Africa, ${ }^{3}$ nfluenza Division, Centers for Disease Control and Prevention, Atlanta, Georgia United States of America, ${ }^{4}$ Influenza Programme, Centers for Disease Control and Prevention-South Africa, Pretoria, South Africa, ${ }^{5}$ Medical Research Council, Respiratory and Meningeal Pathogens Research Unit, Faculty of Health Sciences, University of the Witwatersrand, 'Department of Science and Technology/ National Research Foundation: Vaccine Preventable Diseases, University of the Witwatersrand, Johannesburg, South Africa, ${ }^{7}$ MRC/Wits Rural Public Health and Health Transitions Research Unit (Agincourt), School of Public Health, Faculty of Health Sciences, University of the Witwatersrand, Johannesburg, South Africa, ${ }^{8}$ Centre for Global Health Research, Umeå University, Umeå, Sweden, ${ }^{9}$ INDEPTH Network, Accra, Ghana.

Reducing severe acute respiratory illness (SARI)associated mortality in African children remains a public health priority. We compared the factors associated with mortality among children aged $<5$ years hospitalized with SARI in urban (Chris Hani-Baragwanath Hospital, Soweto) and rural (Matikwana and Mapuleng Hospitals, Mpumalanga) settings in South Africa from 2009-2013. Hospitalized children with SARI enrolled into an active, prospective sentinel surveillance program were eligible. Clinical and epidemiologic data were collected until discharge. Nasopharyngeal aspirates were tested for influenza and nine other respiratory viruses. In-hospital case-fatality proportion (CFP) and risk factors for mortality were determined for each hospital site separately using unconditional logistic regression. The in-hospital CFP was significantly higher in the rural $(6.9 \%, 103 / 1486)$ than the urban $(1.3 \%, 51 / 3811)$ site $(p<0.001)$. This was observed among both HIV-infected (urban: 6.6\%, 17/257) vs. (rural: $12.9 \%, 30 / 233) \quad(p=0.019)$ and HIV-uninfected children (urban: $0.6 \%, 13 / 2236$ ) vs. (rural: $4.2 \% 36 / 857$ ) ( $p<0.001$ ). In the urban site the only factor significantly associated with death on multivariate analysis was HIV infection (odds ratio (OR) 12.1, 95\% confidence interval (CI) 5.8-25.2). In the rural site HIV infection (OR $3.5,95 \% \mathrm{Cl} 1.7-6.9$ ), age $<1$ year (OR 3.5, 95\% $\mathrm{Cl} 2.0-6.1$ ) vs. 1-4 years, any respiratory virus detected (OR $0.4,95 \% \mathrm{Cl} 0.2-0.6$ ), pneumococcal infection on lytA PCR (OR 4.5,95\% Cl 1.8-10.8) and malnutrition (OR 12.8,95\% Cl 1.2-134.6) were associated with mortality. SARI mortality was higher in the rural setting. Efforts to prevent and treat HIV infections in children and reduce malnutrition may reduce SARI deaths.

Key words: SARI, Children, Mortality

\section{ID-P-7: 12-MONTH TREATMENT OUTCOMES AMONGST HIV-POSITIVE ORPHANS AND NON-ORPHANS}

Cheryl Hendrickson', Alana Brennan², Denise Evans ${ }^{1,3}$, Ian Sanne', Sharon Patz ${ }^{4}$, Sanlie Untiedt', Jean Bassett ${ }^{4}$, Matthew P Fox ${ }^{6}$

'School of Clinical Medicine, Department of Internal Medicine, Health Economics and Epidemiology Research Office,

2Department of Global Health, Boston University School of Public Health, Boston, MA, USA, ${ }^{3}$ School of Clinical Medicine, Faculty of Health Sciences, University of Witwatersrand, Johannesburg, South Africa, ${ }^{4}$ Alexandra Clinic, Alexandra, Gauteng, ${ }^{5}$ Witkoppen Health and Welfare Centre, Fourways, Gauteng, ${ }^{6}$ Department of Epidemiology, Boston University School of Public Health, Boston, MA, USA

South Africa has a large orphan population-these children may be more at risk poorer health outcomes. We evaluated the association between orphan status and antiretroviral treatment (ART) outcomes among HIVpositive infants, children and adolescents initiating ART at two public-sector clinics in Johannesburg. We conducted a retrospective cohort study among children (1 month-18 years) initiating standard first-line ART between June 2004May 2013. Modified Poisson regression evaluated the 
association of orphan status with all-cause mortality, loss to follow-Up (LTF; $\geq 3$ months late for a scheduled visit) and detectable viral load ( $\geq 400$ copies $/ \mathrm{ml}$ ) at 12 months on ART. We included 244 (27.1\%) orphans (maternal, paternal or both) and 658 (72.9\%) non-orphans at ART initiation. Median ages were 8.5 years (IQR:5.2-11.6) and 3.0 years (IQR:1.0-7.4) for orphans and non-orphans, respectively. At ART initiation, $36 \%$ were WHO stage III/IV; $17 \%$ had TB. One $(0.4 \%)$ orphan and $16(2.4 \%)$ non-orphans died within one year of ART initiation while $7.8 \%$ and $17.8 \%$ of orphans and non-orphans were LTF. 37 (18.7\%) orphans and $133(29.4 \%)$ non-orphans had a detectable viral load at 12 months post-ART initiation. Adjusted analyses show that orphanhood has a protective effect on the risks of death (RR 0.26:95\% Cl:0.20-0.35), LTF (0.60;95\%Cl:0.44-0.82) and failure to achieve viral suppression $(0.77 ; 95 \% \mathrm{Cl}: 0.70$ 0.84 ) when compared to non-orphans. These results must be analysed further, but may arise from more integrated care for orphans in orphan-specific programming. Understanding the impact of orphan status on ART outcomes could improve targeted strategies, and subsequent treatment and developmental outcomes, for HIV-positive infants, children and adolescents.

Key words: Orphans ART outcomes HIV

\section{ID-P-8: REASONS FOR PARTICIPANT NON-RETURN OF VAGINAL RINGS IN MTN-020/ASPIRE: POTENTIAL ADHERENCE IMPLICATIONS FOR OPEN LABEL EXTENSION STUDIES}

Clare Dott, Krishnaveni Reddy, Helen Rees, Thesla Palanee-Phillips

School of Clinical Medicine, Wits Reproductive Health and HIV Institute (WRHI), University of the Witwatersrand

The ASPIRE trial showed that a dapivirine-containing vaginal ring (VR) was safe and effective in the prevention of HIV infection in women. Women $\geq 25$ years old at baseline had substantial HIV protection (61\%) while those $<25$ had no significant protection (10\%). Higher effectiveness was related to higher adherence. Participants (PTs) received VR and adherence counselling at enrolment and followup visits and were asked to return VR at next visit. We investigated reasons for $V R$ non-return to provide insight into predictors of VR non-use for future open label extension (OLE) studies. VR non-return was considered a protocol deviation (PD). PD data for VR non-return at the Wits RHI site was reviewed to determine reasons supplied by PTs for non-return, age relationships and potential adherence impact. The site enrolled 213 PTs (in follow-up for 12-34 months). Of 4206 prescribed VR, 65 (1.5\%) were not returned timeously. The most common reason was that VR was removed before study visit and lost $(n=15 ; 9$
$\mathrm{PT}<<25$, 6 PTs $\geq 25: 1.5$ times more $\mathrm{PTs}<25)$. VR return was high at the site (98.5\%). Removal before study visits was the most common reason for non-return. This may serve as an indicator of VR acceptability and highlight the impact on adherence. Younger PTs $(<25)$ were more likely to remove and lose VR than older PTs $(\geq 25)$ which is consistent with effectiveness in these groups. Further analyses across all trial sites may help inform better adherence counselling messages and impact adherence in future OLE studies.

Key words: Adherence ASPIRE Ring Prevention

\section{ID-P-9: EFFECT OF AN ECONOMIC INCENTIVE ON UPTAKE OF TB SYMPTOM SCREENING BY HOUSEHOLD CONTACTS OF PATIENTS STARTING TB TREATMENT}

Constance Mongwenyana', Sydney Rosen², Lawrence Long ${ }^{3}$, Given Malete', Machiel Mothapo ${ }^{3}$, Rebecca Berhanu

'School of Clinical Medicine, Faculty of Health Sciences, Clinical HIV Research Unit, University of the Witwatersrand, 'Boston University, ${ }^{3}$ University of the Witwatersrand

Screening household contacts of patients diagnosed with drug-sensitive TB (DS-TB) and drug resistant TB (DR-TB) is a public health priority, but few contacts present at clinics for symptom screening. We conducted a pilot evaluation of the effect of offering an economic incentive to encourage contacts to come to clinics for screening. Adult, confirmed DS-TB or DR-TB patients (index patients) diagnosed at two clinics in Johannesburg were given a referral card offering screening and a reward for each adult and child living in the index patient's household. Each contact who came to the clinic for screening received a shopping voucher worth R50 (DS-TB) or R100 (DR-TB). The index patient also received a voucher (R100) for participation. The primary outcome was the proportion of contacts screened $\leq 30$ days of index subject enrollment. 96 index patients (56 DS-TB, 40 DR-TB, 73\% HIV-infected) were enrolled. Among the 104 reported household contacts of DS-TB index patients, 24 (23\%) presented for symptom screening, from 16/56 (29\%) index patient households. Among the 101 reported household contacts of DR-TB index patients, 33 (33\%) presented for symptom screening, from 15/40 (38\%) index patient households. Of contacts screened, $46 \%$ were children $<18$ years and $26 \%$ $\leq 10$ years; overall, $33 \%(26 / 79)$ of reported child contacts were screened. Suggested reasons for not presenting included TB/HIV stigma, transport costs for contacts, and, for DR-TB patients, prior screening at a local PHC. Offering a small shopping voucher incentive resulted in a modest proportion of household contacts presenting for TB symptom screening.

Key words: TB, Contacts, Screening, Incentive 


\section{ID-P-10: COMPARISON OF TRADITIONAL CULTURE AND QPCR FOR DETECTION OF PNEUMOCOCCAL COLONIZATION BY PNEUMOCOCCAL CONJUGATE VACCINE (PCV) STATUS IN AFRICAN CHILDREN.}

\author{
Courtney Olwagen', Peter V. Adrian², Shabir A. Madhi
}

'School of Pathology, Respiratory and Meningeal Pathogens Research Unit, University of the Witwatersrand, '2Department of Science and Technology/National Research Foundation: Vaccine Preventable Diseases, University of the Witwatersrand, ${ }^{3}$ National Institute for Communicable Diseases: a division of National Health Laboratory Service, Johannesburg, South Africa

PCV immunization of children induced shifts in circulating pneumococcal serotypes. The study aimed to compare traditional culture methods and $\mathrm{QPCR}$ for the detection of pneumococcal colonization in PCV-vaccinated and PCV-unvaccinated children, and evaluate the effect of infant vaccination with 7-valent PCV (PCV7) on vaccineserogroup pneumococcal carriage. We used a multiplex quantitative PCR (qPCR) to evaluate vaccine-serotype and non-vaccine serotype nasopharyngeal colonization in archived swabs of PCV-vaccinated and PCVunvaccinated Black-African children, at 9 and 16 months of age prior to the routine immunization of children with PCV. We showed good concordance between culture and $\mathrm{QPCR}$ for the detection of overall pneumococcal carriage, with GPCR being significantly more sensitive at 9 months $(77.2 \%$ vs $65.4 \% ; p=0.004)$ and 16 months $(78.4 \%$ vs $65 \%$; $p<0.001$ ) of age. Furthermore, $q P C R$, identified the reduction in VT serotypes $(p<0.001)$ and a corresponding increase in NVT serotypes $(p<0.001)$. The increase in NVT carriage was driven by a limited number of serotypes such as 19A, where proportions increased at 9 and 16 months respectively. Molecular detection of pneumococcal serotypes was more sensitive than culture and allowed us to detect multiple serotypes. The ability of GPCR to detect pneumococcus at a low carriage density, will allow us to gain a better understanding of serotype carriage and its impact in disease prevalence.

Key words: Streptococcus pneumoniae, vaccine effect, serotype carriage, PCV

\section{ID-P-11: ASSOCIATION OF PNEUMOCOCCAL CONJUGATE VACCINE (PCV) IMMUNIZATION AND DENSITY OF NASOPHARYNGEAL BACTERIAL COLONIZATION USING A MULTIPLEX QUANTITATIVE POLYMERASE CHAIN REACTION ASSAY}

Courtney Paige Olwagen', Peter V. Adrian², Shabir A. Madhi

'School of Pathology, Respiratory and Meningeal Pathogens Research Unit, University of the Witwatersrand, ${ }^{2}$ Department of Science and Technology/National Research Foundation: Vaccine Preventable Diseases, University of the Witwatersrand, ${ }^{3}$ National Institute for Communicable Diseases, National Health Laboratory Service, Johannesburg, South Africa
Nasopharyngeal colonization is a pre-requisite for developing bacterial mucosal and invasive disease. Immunization of children with PCV reduces their risk of colonization by vaccine-serotypes, which could affect the microbiome of the nasopharynx. We evaluated the association of 7-valent PCV immunization on the prevalence density of nasopharyngeal colonization by common, potentially pathogenic bacteria. We used multiplex quantitative PCR to evaluate nasopharyngeal colonization by 7-valent PCV serotypes, non-vaccine serotypes, Haemophilus influenzae, Staphylococcus aureus, Moraxella catarrhalis, and Neisseria meningitis in PCV-vaccinated and PCV-unvaccinated, Black-African children. PCV vaccination was associated with a decline in overall prevalence of pneumococcal colonization, whilst the prevalence of $H$. influenzae and $S$. aureus colonization increased. Furthermore, the density of pneumococci, $\mathrm{H}$. influenzae, M. catarrhalis and S. aureus was higher among PCV-vaccinated compared to PCV-unvaccinated children at 9 months age, although, this difference diminished with increasing age. PCV immunization was associated with reduction in PCV7-serotype colonization, but increased the prevalence and density of colonization by other bacterial species. The clinical relevance of this needs further exploration in relation to disease outcomes.

Key words: Streptococcus pneumoniae, PCV, Vaccine effect, Carriage Density

\section{ID-P-12: ADOLESCENTS AND YOUNG ADULTS ON FIRST-LINE ART PREFER A SELF-ADMINISTERED ELECTRONIC QUESTIONNAIRE TO ASSESS ART ADHERENCE}

Denise Evans 1,2 , Kamban Hirasen², Rebecca Berhanu ${ }^{3}$, Rita Graber ${ }^{3}$, Julia Turner ${ }^{3}$, Lawrence Long ${ }^{2}$, Matthew P. Fox ${ }^{4}$

'School of Clinical Medicine, Department of Internal Medicine, ${ }^{2} \mathrm{Health}$ Economics and Epidemiology Research Office, ${ }^{3 R i g h t ~ t o}$ Care, ${ }^{4}$ Boston University School of Public Health

Because many young South African's have been exposed to mobile technology we set out to determine if a self-administered electronic questionnaire would be an acceptable approach to measure adherence to ART. This was a cross-sectional study among HIV positive adolescents and young adults (18-35 years) on firstline ART for $>3$ months at the Themba Lethu HIV Clinic in Johannesburg, South Africa between 07/2015-01/2016. Participants were asked a series of questions to assess their preference for a self-administered electronic versus a counsellor/social worker administered paper-based questionnaire to measure adherence to ART. Of the 77 participants enrolled (14\% refusal rate), 19 (25\%) were male and the majority (88\%) had been on ART for more than 12 months. 66\% (51/77) reported good adherence, defined by a VAS score $\geq 90 \%$. When using a five-level Likert scale, $80 \%$ (41/51) agreed or strongly agreed that they are 
comfortable using a Smartphone or tablet. 60\% (46/77) reported that they preferred an electronic questionnaire on a Smartphone or tablet over a paper form and $68 \%$ (52/77) preferred a self-administered questionnaire over an interview-administered one. Finally, $95 \%$ (38/40) of study participants who completed the adherence questionnaire on an electronic device reported that it was relatively easy to complete. Conversely, 52\% (40/77) had concerns about confidentiality and that their responses would not be stored and transferred safely on an electronic device. In order for self-administered electronic questionnaires to be useful in routine clinic settings participants need to be reassured that their responses will be securely stored and transferred to maintain patient confidentiality.

Key words: HIV, adherence, adolescents and young adults,

\section{ID-P-13: EARLY OUTCOMES OF THIRD- LINE ANTIRETROVIRAL THERAPY AT A LARGE PUBLIC-SECTOR CLINIC IN JOHANNESBURG, SOUTH AFRICA}

Denise Evans ${ }^{1,2}$, Kamban Hirasen², Rebecca Berhanu3, Prudence Ive ${ }^{4}$, David Spencer ${ }^{3}$, Sharlaa Badal-Faesen ${ }^{4}$, In Sanne ${ }^{4}$, Matthew P. Fox ${ }^{5}$

'School of Clinical Medicine, Department of Internal Medicine, University of the Witwatersrand, ${ }^{2} \mathrm{Health}$ Economics and Epidemiology Research Office, ${ }^{3}$ Right to Care, ${ }^{4}$ Clinical HIV Research Unit, ${ }^{5}$ School of Public Health, Boston University

There is limited data on outcomes of third-line ART in subsaharan Africa. To identify predictors of switch to thirdline we conducted a retrospective analysis of adults ( $\geq 18$ years) who initiated standard second-line ART between 04/2012-11/2013. Patients were followed from start of second-line until the earliest of switch to regimen containing etravirine, raltegravir or darunavir after 08/2012, death, loss to follow up, transfer or administrative censoring (11/2014). For virologic response we restricted the analysis to patients starting third-line ART between 08/2012-05/2014 and defined viral load suppression as a viral load $<400$ copies $/ \mathrm{ml}$. Of the patients that initiated second-line ART ( $n=3144), 32$ (1\%) initiated third-line ART. Predictors of switch included gender (male vs. female; HR $2.2895 \% \mathrm{Cl} 1.11-4.67)$, higher viral load at secondline initiation ( $\geq 50000$ vs. $<1000$ copies/ml; HR $4.6195 \% \mathrm{Cl}$ 1.78-11.96) and not suppressing at the first viral load after second-line initiation (HR $3.5595 \% \mathrm{Cl}$ 1.71-7.37). By the end of follow-up (median 13.1 months), 15 patients (46.9\%) had suppressed their viral load in a median time of 4.6 months, $3(9.4 \%)$ had not suppressed, $5(15.6 \%)$ did not have a repeat viral load, 1 (3.1\%) had died, 1 (3.1\%) was loss to follow-up and 7 (21.9\%) transferred to another facility. Close to $50 \%$ of patients on third-line suppressed their viral load although high rates of transfer and limited follow-up time may have restricted repeat viral load testing and underestimated suppression rates.

Key words: Third-line, ART, HIV, Treatment Outcomes

\section{ID-P-14: MYCOBACTERIAL DD CARBOXYPEPTIDASES: FILLING IN THE GAPS}

Ditshego Ralefeta ${ }^{1,2}$, Bavesh Kana², Edith Machowski²

'School of Pathology, Department of Molecular Medicine and Haematology, University of the Witwatersrand, ${ }^{2}$ Centre of

Excellence for Biomedical TB research

Penicillin Binding Proteins (PBPS) have formed the basis of successful antibiotic therapy for decades but these proteins remain largely uncharacterized in Mycobacterium tuberculosis, the causative agent of tuberculosis (TB). In this study we aimed at characterizing the physiological function of three low molecular weight PBPs, termed DD-carboxypeptidase (encode by Rv2911, Rv3627c and Rv3330), in Mycobacterium tuberculosis through heterologous expression in Mycobacterium smegmatis, a model organism routinely used for TB research. Cellular localization studies with rseGFP $\mathrm{N}$-terminally tagged derivatives of these proteins revealed localization at both poles with multiple foci either at mid-cell or three-quarter position, suggesting that they play a role in temporal and spatial co-ordination of cell division. Over-expression altered colony morphology, resulting in smooth colonies with a mucoid surface and cording defects. Increased expression resulted aberrant biofilm formation and also affected bacterial sliding on semi-solid agar, indicative of an alteration in the threedimensional packing of cells in a community or changes in membrane properties. Deregulated expression of Rv2911 and Rv3627c caused alterations in cell length and cell wall morphology accompanied by an increase in inclusion bodies. Collectively, our data point to important, possibly redundant roles for the multiple DDcarboxypeptidases-encoding genes in Mycobacterium tuberculosis and highlights these proteins as possible new drug targets for TB.

Key words: Mycobacterium tuberculosis, Tuberculosis, Penicillin Binding Proteins, DD-Carboxypeptidases

\section{ID-P-15: PRODUCTION OF PEGYLATED HELPER DEPENDENT ADENOVIRAL VECTORS EXPRESSING ANTI-HBV PRI- MIR SEQUENCES FROM A LIVER SPECIFIC PEPCK PROMOTER}

Duadane' Smit, Betty Maepa, Patrick Arbuthnot

School of Pathology, Department of Haematology and Molecular Medicine, Antiviral Gene Therapy Research Unit, Faculty of Health Science, University of Witwatersrand

Hepatitis B virus is a global health problem and accounts for the chronic infection of approximately 350 million individuals worldwide, with high incidence found in areas such as Asia and Africa. Current treatments are limited by the emergence of viral resistance and adverse side effects. These challenges prompted the development 
of a new hepatitis B therapy. Studies using gutless/Helper Dependent Adenoviral vector (HDAd) expressing anti$\mathrm{HBV}$ sequences from cytomegalovirus (CMV) promoter showed in vivo silencing of HBV replication. Nevertheless, HBV inhibition was short lived and might have been as a result of promoter inefficiency and/or immune stimulation by vector $\beta$-galactosidase marker and the vector capsid epitopes. To lengthen HBV inhibition period, lac Z (encode $\beta$-galactosidase) deficient and capsid modified HDAd vectors expressing anti-HBV sequences from Phosphoenolpyruvate carboxykinase (PEPCK) promoter are used in this study. Plasmid constructs required for production of HDAds were successfully constructed and characterized. Currently, HDAd vectors are being produced in tissue culture. This will be followed by in vitro and in vivo determination of anti-HBV sequence expression, HBV gene knockdown and safety. Hence, this study focuses on improving the duration of HBV silencing by anti-HBV HDAd vectors, making them more efficient and relevant for chronic HBV infection treatment.

Key words: HBV, HDAd, PEPCK, pri-miR

\section{ID-P-16: USING SEROLOGICAL ASSAY TO ESTIMATE THE RISK FACTORS ASSOCIATED WITH PLASMODIUM FALCIPARUM EXPOSURE IN BUSHBUCKRIDGE MUNICIPALITY, MPUMALANGA PROVINCE, SOUTH AFRICA}

Eunice Agubuzo', Joseph Biggs², Jackie Cook², Jaishree Raman³, Natashia Morris ${ }^{5}$, Khumbulani Hlongwana ${ }^{6}$, Ishen Seocharan, ${ }^{5}$ Maureen Coetzee ${ }^{1,3}$, Immo Kleinschimd ${ }^{2,1}$, Aaron Mabuza ${ }^{7}$

'School of Pathology, Wits Research Institute for Malaria (WRIM), ${ }^{2}$ Department of Infectious Disease Epidemiology, London School of Hygiene and Tropical Medicine, London, UK, ${ }^{3}$ Centre for Opportunistic, Tropical and Hospital Infections, National Institute for Communicable Diseases, Johannesburg, South Africa, ${ }^{4}$ Wits Research Institute for Malaria, School of Pathology, Faculty of Health Sciences, University of the Witwatersrand, Johannesburg, South Africa, ${ }^{5}$ Medical Research Council, Durban, South Africa, UUniversity of Kwazulu/Natal, Durban, South Africa, 'Department of Health and Social Services, Mpumalanga Provincial Government, Nelspruit, South Africa

Malaria transmission intensity in South Africa has reached the WHO recommended pre-elimination threshold. Monitoring the effectiveness of current controlinterventions such as entomological inoculation rate and parasite rate is proving challenging. In this study we evaluated the potential of using sero-prevalence data to identify potential risk factors associated with low transmission in low transmission setting. Blood spots and demographic data were collected from 2,897 individuals across 28 study clusters in Bushbuckridge between April and June, 2015. Sera eluates from blood spots were assayed for human anti-malaria antibodies against $P$. falciparum AMA-1 and MSP-1 ${ }_{19}$ antigens, using ELISA. Of the individuals surveyed
$21.3 \%$ were sero-positive for AMA-1, 16.3\% for MSP-119, and $31.4 \%$ sero-positive for both antigens. The odds of being sero-positive increased with age $(p<0.001)$ and individual with no formal secondary schooling $(p=0.038)$. No association between sero-positivity and travel ( $p=0.309)$, out-door night-time activities ( $p=0.259)$, employment status $(p=0.902)$ or vector control measures $(p=0.815)$ was found. Sero-positivity to AMA-1 was higher than sero-positivity to MSP-119. This correlates with a higher sero conversion rate for AMA-1 compared to MSP-1. Poor educational status was identified as a potential risk factor for malaria exposure, however due to reverse causality, more needs to be revealed to determine whether poor education causes malaria exposure or does malaria exposure result in attaining a poor level of education. Data from this study supports the use of serology to monitor malaria transmission in low transmission South African settings.

Key words: ELISA, Serology, Risk factors, Plasmodium falciparum

\section{ID-P-17: A REVIEW OF THE STERILE INSECT TECHNIQUE FOR THE CONTROL OF MALARIA VECTORS IN SOUTH AFRICA}

Givemore Munhenga ${ }^{1,2}$, Basil B Brooke ${ }^{1,2}, K_{0}$ bus Slabbert ${ }^{3}$, Alan Kemp ${ }^{2,4}$, Leonard C Dandalo', Oliver R Wood', Leanne N Robertson ${ }^{1}$, Maria Kaiser ${ }^{1}$, Lizette L Koekemoer ${ }^{1}$

'School of Pathology, Wits Research Institute for Malaria (WRIM),Faculty of Health Sciences, University of the Witwatersrand, Johannesburg, South Africa, ${ }^{2}$ Centre for Opportunistic, Tropical and Hospital Infections, National Institute for Communicable Diseases, Private Bag X4, Sandringham, Johannesburg, South Africa, 3iThemba LABS (Laboratory for Accelerator Based Sciences), Somerset West, South Africa, ${ }^{4}$ Special Pathogens Unit

The backbone of malaria control in South Africa is vector control, mainly achieved through the insecticide based indoor residual spraying (IRS) technique. However, the effectiveness of IRS is under threat owing to the development of insecticide resistance in target malaria vector populations. Additionally, IRS is less effective at controlling the behaviourally variable vector Anopheles arabiensis, which is responsible for low-level, seasonal malaria transmission in South Africa. Against this background there was a need to identify additional vector control interventions to supplement existing strategies. The use of the Sterile Insect Technique (SIT) has been proposed. A multi-year plan for testing the feasibility of SIT for malaria control in South Africa was developed by a consortium representing several partners in 2010 . Over the last five years pre-feasibility studies have been carried out culminating in the identification of a pilot release site, and the development and characterisation of an An. arabiensis genetic sexing strain. Intensive baseline vector surveillance at the release site has yielded important 
information concerning the population dynamics of the target An. arabiensis population. The purpose of this presentation is to review the experience and knowledge gained in developing the SIT as a malaria vector control tool and to focus on the challenges and current status of the project. An insight into the pending pilot release phase of the project is also provided

Key words: Malaria vector control, Sterile Insect Technique, Anopheles arabiensis, South Africa

\section{ID-P-18: PREVENTION OF MOTHER-TO- CHILD TRANSMISSION (PMTCT) IN SOUTH AFRICA: LATE INITIATION AND LOW LEVELS OF COVERAGE PERSIST DESPITE SHIFT FROM OPTION A TO OPTION B}

Given Malete', Sydney Rosen², Mhairi Maskew³, Lawrence Long ${ }^{3}$, Constance Mongwenyana ${ }^{3}$, Cynthia Nyoni, Hazel Tau

'School of Clinical Medicine, Faculty of Health Science, University of the Witwatersrand, Health Economics and Epidemiology

Research Office, ${ }^{2}$ Boston University, ${ }^{3}$ Wits University

In 2013, South Africa replaced PMTCT Option A (AZT at CD4>350; HAART at CD4<350) with Option B (HAART for all using a fixed-dose combination tablet taken once-daily). Guidelines for both Option A and Option B called for initiation of PMTCT by 14 weeks' gestation and 24 weeks of PMTCT adherence before delivery. In a pre/post cohort study in Johannesburg, we compared adherence to recommended PMTCT guidelines for Option A (retrospective record review) and Option B (prospective enrollment) for HIV-infected pregnant women presenting for their first antenatal booking visit. We enrolled 151 subjects in Option A and 154 in Option B. Both groups presented for antenatal care late, at a median of 22 weeks (IQR 18-27). Only 12\% in Option A and 15\% in Option B initiated PMTCT by the recommended 14 weeks, though Option B presented with higher CD4 counts (median 366;IQR 252-513) than Option A (295; 200-466). In Option B, $42 \%$ had documented possession of PMTCT drugs for the recommended 24 weeks compared to $36 \%$ in Option A. Very few women had complete PMTCT drug coverage during the final 4 months of pregnancy $17 \%$ and $5 \%$ in Option A and Option B, respectively). Fully $40 \%$ of Option A and $44 \%$ of Option B had no recorded ARV drug possession during the final 4 months at all. Despite an increase in presenting CD4 count, the introduction of Option B saw little change in gestational age at ANC booking and persistence of poor PMTCT coverage, particularly in the critical period prior to delivery. Further action is needed to promote earlier initiation of and adherence to PMTC.

Key words: HIV, PMTCT, ANC, ARV

\section{ID-P-19: OPTIMISATION OF A CHROMATIN IMMUNOPRECIPITATION METHOD TO INVESTIGATE EPIGENOME CHANGES EFFECTED BY TRANSCRIPTION ACTIVATOR- LIKE EFFECTOR REPRESSORS}

Ilke Roelofse, Kristie Bloom, Abdullah Ely, Buhle Moyo, Patrick Arbuthnot

Wits/SAMRC Antiviral Gene Therapy Research Unit, Department of Molecular Medicine and Haematology, School of Pathology

Covalently closed circular DNA (cccDNA) persists inside the hepatocyte during chronic hepatitis B virus (HBV) infection. As current anti-HBV treatment does not target this episomal DNA, viral replication can resume. This makes cccDNA an attractive target for novel therapies. Our group engineered transcription activator-like effector repressors (rTALEs) that contain a Krüppel-associated box transcriptional repressor domain, resulting in targeted HBV transcriptional repression through interaction with histonemodifying proteins. We have established rTALE-binding of cccDNA, recorded efficient HBV silencing in vivo and in vitro, and identified changes to the methylation of HBV DNA. As methylated genomic regions are generally associated with inactive loci, the aims of this study are to utilise chromatin immunoprecipitation (ChIP) to establish (1) whether rTALE methylated regions correlate with constitutive heterochromatin, and (2) if a reduction in transcriptional machinery is recorded following rTALE treatment. Histone modifications are key epigenetic chromatin regulators. Posttranslational changes to the core histone $\mathrm{H} 3$ by trimethylation at lysine 9 or 27 will result in transcriptionally inactive, condensed heterochromatin. We will probe the modifications effected by the rTALEs through selective enrichment by immunoprecipitation using antibodies specific for rTALEs, heterochromatin and RNA polymerase II. Despite its utility, this protocol is complex and the assay therefore requires intensive optimisation for each experimental condition. Nevertheless it is a powerful method that should enable detailed analysis of the chromatin status of HBV cccDNA. We report a ChIP method for assessing HBV-targeted viral epigenome modification in vitro.

Key words: HBV, Epigenome Editing, ChIP

\section{ID-P-20: SPECT BRAIN IMAGING IN HIV INFECTED INDIVIDUALS WITH NEW ONSET SEIZURES}

Kapila Hari', Girish Modi', Mboyo-di-Tambo Vangu ${ }^{3}$

'School of Clinical Medicine, Department of Internal Medicine, University of the Witwatersrand, '2Department of Neurology, WITS, ${ }^{3}$ Department of Radiation Sciences, WITS

We present here the role of SPECT brain imaging HIV infected individuals with new onset seizures (NOS). These were patients in whom no cause was identifiable at baseline. Fifteen patients with NOS and no identifiable cause participate in this long-term study. They had 
repeated clinical assessments, laboratory investigations,

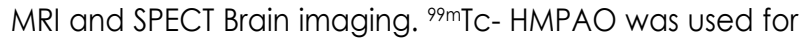
brain SPECT imaging and the SPECT scans were repeated at 6-monthly intervals for the duration of the patients' inclusion in the study. We used PMOD base functionality version 3.6 (PBAS 3.6) for quantitative assessment of counts within automatic generated regions of interest (ROIs). There were 10 female and 5 male participants aged between 20-55 years. Five participants were on highly active anti-retroviral therapy (HAART) at baseline and 9 of the remaining 10 were eligible for HAART. Cerebral hypo perfusion on SPECT was noted at baseline mainly in the frontal, cingulate and temporal regions. Significant improvement of cerebral perfusion $(p=0.003)$ was seen in the follow up SPECT scans in 10 of 11 patients with repeated scintigraphy imaging, but more pronounced in 4 participants who initiated HAART during the study $(p=0.001)$. New onset of seizures may represent early stages of the spectrum of neurocognitive dysfunction seen in HIV infected individuals without identifiable cause. Because these individuals are likely to benefit from the initiation of HAART, the introduction of SPECT brain imaging in their follow up may play a role in the assessment of HAART related cognitive improvement.

Key words: HIV, SEIZURES, PECT, SCANS

\section{ID-P-21: MOLECULAR CHARACTERIZATION OF INVASIVE CAPSULE NULL NEISSERIA MENINGITIDIS IN SOUTH AFRICA}

Karistha Ganesh ${ }^{1,2}$, Mushal Allam', Nicole Wolter ${ }^{1,2}$, Holly B. Bratcher ${ }^{3}$, Odile B. Harrison ${ }^{3}$, Jay Lucidarme', Ray Borrow ${ }^{4}$, Linda de Gouveia', Susan Meiring', Monica Birkhead', Martin C. J. Maiden $^{3}$, Anne von Gottberg ${ }^{1,2}$ and Mignon du Plessis ${ }^{1,2}$

' National Institute for Communicable Diseases of the National Health Laboratory Service, Johannesburg, South Africa, ${ }^{2}$ School of Pathology, Faculty of Health Sciences, University of the Witwatersrand, Johannesburg, South Africa, ${ }^{3}$ Department of Zoology, University of Oxford, Oxford, United Kingdom, ${ }^{4}$ Meningococcal Reference Unit, Public Health England, Manchester Medical Microbiology Partnership, Manchester Royal Infirmary, United Kingdom

Encapsulation is an important virulence determinant for invasive meningococcal disease (IMD). Non-groupable (NG) meningococci containing the capsule null locus (cnl) lack capsule biosynthesis genes in the capsular polysaccharide synthesis locus. Rare cases of IMD by $\mathrm{cnl}$ isolates belonging to sequence types (ST)-845, ST-198 and ST-192 have been described, whilst ST-53 is associated with carriage. From 2003-2013, 4770 cases of IMD in South Africa were reported through national, laboratory-based surveillance, of which 2988 (63\%) had viable isolates. Five isolates were NG and genomic data were obtained and deposited in PubMLST.org/neisseria, enabling strain designations to be determined. This revealed that four of these isolates were $\mathrm{cnl}$. The first strain (NG:P1.7-2,30:F1-2:ST-53(cc53)) contained chl1 , and caused empyema in a 46-year-old male with history of previous tuberculosis, smoking and diabetes mellitus (2006). Three isolates were NG:P1.18-1 1,42-2:FA:ST192(cc 192) and contained cnl-2. One isolate caused meningitis in 2010 in a 15-year-old male with unknown clinical background. The remaining two isolates (2011 and 2012) were from recurrent IMD episodes in a 6-yearold male with complement 6 deficiency. ST-53 possessed alleles for NHBA peptide 191 and fHbp variant 2; whilst ST-192 contained NHBA peptide 704 and fHbp variant 3. All four isolates lacked nadA. The isolates were closely related to other ST-53 and ST-192 cnl isolates found on PubMLST. These data are consistent with susceptibility to cnl IMD being influenced by clinical background. This is the first report of $c n l$ IMD in South Africa.

Key words: Neisseria meningitidis, cnl

\section{ID-P-22: CONDITIONAL CASH TRANSFER TO PREVENT HIV INFECTION AMONG YOUNG WOMEN IN SOUTH AFRICA}

Kathleen Kahn', Audrey Pettifor ${ }^{2,4}$, Catherine MacPhail', Amanda Selin'2, Xavier Gomez-Olive ${ }^{4}$, Wonderful Mabuza ${ }^{4}$, Immitrude $^{2}$ Mokoena ${ }^{4}$, Ryan Wagner ${ }^{4}$, Stephen Tollman et. $a^{4}$

'School of Public Health, Rural Public Health and Health Transitions Research Unit (Agincourt), ${ }^{2} \mathrm{UNC},{ }^{3} \mathrm{MRC} /$ Wits-Agincourt Unit, Univ New England, Australia, ${ }^{4}$ MRC/Wits-Agincourt Unit

Young South African women are at high risk of HIV infection, fuelled by structural factors including schooling, socio-economic status (SES) and financial dependence on partners. Cash transfers may reduce HIV risk by addressing these factors. This phase III individually randomized trial assessed the impact of a conditional cash transfer on HIV acquisition in young women. From April 2011 to March 2015, young women and their parent/guardian in the intervention arm received a monthly cash transfer conditional on $80 \%$ school attendance, verified using school rosters. Participants were aged 13-20, in high school, not married or pregnant, and resident in the Agincourt Health and Socio-Demographic Surveillance System site in rural Mpumalanga. Assessments were at baseline, then annually for up to three follow-up visits. HIV and HSV-2 testing were conducted, with an interview using Audio Computer Assisted Self Interviewing (ACASI). This assessed sexual behaviour, schooling, mental health, SES and gender dynamics. To compare treatment arms, time to first HIV detection was analysed using a Cox proportional hazards model. Results showed that staying in school and greater school attendance significantly reduced the risk of HIV. Young women receiving cash reported fewer sex partners, less unprotected sex and experienced less intimate partner violence. The cash transfer did not, however, reduce new HIV infections. Cash transfers are increasingly offered to young women to reduce HIV risk in sub-Saharan Africa. School attendance was high in both arms suggesting that conditioning is not needed in the South African context. Cash transfers may be protective for high risk sub-groups.

Key words: HIV, prevention, conditional cash transfer, RCT 


\section{ID-P-23: NON-VACCINE STREPTOCOCCUS PNEUMONIAE SEROTYPES CAUSING INVASIVE DISEASE IN CHILDREN <5 YEARS PRE AND POST CONJUGATE VACCINE INTRODUCTION IN SOUTH AFRICA}

Kedibone M. Ndlangisa ${ }^{1,2}$, Mignon du Plessis ${ }^{1,2}$, Linda de Gouveia', Mushal Allam', Nicole Wolter ${ }^{1,2}$, Keith P. Klugman ${ }^{2.3}$, Cheryl Cohen ${ }^{1,2}$, Susan Mering', Rebecca A. Gladstone ${ }^{4}$, Stephen D. Bentley ${ }^{4}$, Lesley McGee ${ }^{5}$, Robert F. Breiman ${ }^{6}$, and Anne von Gottberg ${ }^{1,2}$, for GERMS-SA

'National Institute for Communicable Diseases (NICD), a division of the National Health Laboratory Service, Johannesburg. South Africa, ${ }^{2}$ School of Pathology, Faculty of Health Sciences, University of the Witwatersrand, Johannesburg, South Africa, 3Hubert Department of Global Health, Rollins School of Public Health, Division of Infectious Diseases, School of Medicine, Emory University, Atlanta, Georgia, USA, "Pathogen Genomics, Wellcome Trust Sanger Institute, Hinxton, UK, ${ }^{5}$ Centers for Disease Control and Prevention, Atlanta, USA, ${ }^{6}$ The Emory Global Health Institute, Atlanta, USA

In South Africa, the 7-valent pneumococcal conjugate vaccine (PCV7) was introduced in 2009 and replaced with PCV13 in 2011. We describe nonPCV serotypes causing invasive pneumococcal disease (IPD) in children $<5$ years. We reviewed cases reported as part of on-going, national laboratory-based IPD surveillance, including available MLST data for nonPCV serotypes $(8,12 \mathrm{~F}$ and $35 \mathrm{~B})$ that appeared to increase post PCV. Average incidence for the periods 2005-2008 (prePCV) and 2012-2015 (postPCV) was calculated. From 2005-2015, 10,569 (26\%) of 40,517 IPD cases reported were in children. Overall IPD incidence declined by $73 \%$ : 115 to $31 / 100,000$ population $(p<0.0001)$; while nonPCV13 incidence increased $7 \%, 3.2$ to $3.4 / 100,000(p=0.2)$. Serotypes 8 and $12 F$ incidence increased $32 \%(0.37$ to $0.54 / 100,000, p=0.1)$ and $30 \%$ (0.25 to $0.36 / 100,000, p=0.2)$, respectively. Predominant genotypes did not change pre and post PCV [serotype 8:CC53 (13/14 and 44/44, respectively), 12F:CC989 (4/10 and $17 / 26$, respectively) and CC2416 $/ 6 / 10$ and $9 / 26$, respectively)]. Serotype 35B incidence increased $77 \%$ : 0.08 to $0.34 / 100,000$ ( $p=0.001$ ). No prePCV isolates were sampled for genotyping, but post PCV, the majority of 35B isolates were CC361 (23/30, 78\%). Non-significant increases in serotypes 8 and $12 F$ in children appeared to be due to expansion of genotypes that existed pre PCV, one of which appears to be unique to South Africa (12F:CC2416). The 35B/CC361 combination is worth exploring as pubmlst. net shows that this genotype is associated with other serotypes.

Key words: Invasive pneumococcal disease, 7-valent pneumococcal conjugate vaccine, non-PCV serotypes

\section{ID-P-24: EPIDEMIOLOGICAL CHARACTERISTICS AND RATE OF CLOSTRIDIUM DIFFICILE INFECTION IN GAUTENG STATE HEALTH-CARE FACILITIES}

Larissa Singh ${ }^{1,2}$, Desmond Schnugh ${ }^{1,2}$, Karren Le Roux ${ }^{1,2}$

'Infection Control Services, Charlotte Maxeke Johannesburg Academic Hospital, National Health Laboratory Service,
${ }^{2}$ School of Pathology, Department of Clinical Microbiology and Infectious Diseases, University of the Witwatersrand

Clostridium difficile is an, anaerobic spore-forming bacillus that is emerging as a leading cause of life-threatening, healthcare-acquired infections worldwide. We aim to study the incidence rates and some epidemiological characteristics of Clostridium difficile infection in the following state hospitals in Gauteng; Charlotte Maxeke Johannesburg Academic Hospital (CMJAH), Yusuf Dadoo Edenvale, Far East Rand, Leratong, Hillbrow Clinic, Natalspruit, Sebokeng, Sizwe Tropical Diseases Hospital, South Rand, Tambo Memorial and Tembisa. 1391 fresh stool samples for the period 1 January-31 December 2015 were assayed for toxin B, binary toxin and the tcdC deletion, using real time PCR detection on the GeneXpert system (Cepheid). Samples were analysed according to the following criteria: presence of C.difficile infection, B1/ NAP1/027 hypervirulent strain, patient age, gender, ward, hospital, and incidence rate per ward and per hospital. Results were compared to a similar 2014 study. There was a decrease in the average age to 31.41 years with a range from 6 days to 91 years old. The 2015 study indicated a higher number of samples referred for C.difficile testing but a decrease in the incidence rate to $22.21 \%$. No samples tested positive for the hypervirulent NAPI strain of $C$. difficile. The male to female ratio was $0.81: 1$, with a higher incidence in females. At CMJAH, the infectious diseases ward showed a high incidence rate. This result follows a similar trend to the 2014 study.

Key words: Clostridium difficile

\section{ID-P-25: DEVELOPMENT OF A GENETIC SEXING STRAIN (GSS) OF ANOPHELES ARABIENSIS (DIPTERA: CULICIDAE) FOR KWAZULU NATAL, SOUTH AFRICA.}

Leonard Dandalo', Givemore Munhenga ${ }^{1,2}$, Maria Kaiser ${ }^{1,2}$ Lizette L. Koekemoer ${ }^{1,2}$

'School of Pathology, Faculty of Health Sciences, University of the Witwatersrand, Wits Research Institute for Malaria, University of the Witwatersrand, ${ }^{2}$ Center for Opportunistic, Tropical and Hospital Infections, National Institute for Communicable Diseases, Sandringham, Johannesburg, South Africa.

Mosquito Sterile Insect Technique (SIT) programme requires the release of sterile males only. Females are not accepted because they transmit malaria and cannot contribute to population reduction. This, therefore, necessitated the development of an efficient sex separation system prior to the project implementation. Female An. arabiensis mosquitoes originating from KwaZulu Natal, SA named KWAG were mated with males obtained from a locally modified genetic sexing strain (GSS) called GAMA. The resultant offspring were backcrossed to KWAG females to obtain males carrying dieldrin resistance on the Y chromosome and a genotype representing the wild KwaZulu Natal population. Life history characteristics of the 
newly developed strain denoted GMK and the parental strains were investigated. In addition to this the reduction of dieldrin waste were investigated. The GMK strain showed semi-sterility due to chromosomal rearrangement as exhibited by very low egg hatch rates compared to the parental strains. The egg hatch rates were $27.3 \%$ for $\mathrm{FO}$ and $19.2 \%$ for $\mathrm{Fl}$ generations compared to $42 \%$ and $78.7 \%$ for GAMA and KWAG respectively. The strain showed a faster development in immature stages and adult survivorship compared to the parental strains. Dieldrin waste can be minimised by maximising the number of eggs treated to produce male offspring. The establishment of a local GSS of An. arabiensis with a local genetic background was successful.

Key words: Genetic Sexing Strain, Sterile Insect Technique, GMK

\section{ID-P-26: DACB IS ESSENTIAL IN MYCOBACTERIUM SMEGMATIS}

Lethabo Mashigo ${ }^{1,2}$, Christopher Shawn Ealand², Bavesh Davandra Kana ${ }^{2}$

ISchool of Pathology, Faculty of Health Sciences, University of the Witwatersrand, Johannesburg, South Africa, ${ }^{2}$ DST/NRF Centre of Excellence for Biomedical TB Research

Tuberculosis (TB) is a bacterial infection caused by Mycobacterium tuberculosis. Although responsive to drug treatment, TB still remains a global problem, due to the emergence of drug resistant strains, which has led to the need for greater understanding of bacterial physiology and novel chemotherapeutic options. As a direct result of its inherent complexity, the mycobacterial cell wall has emerged as a resource for the identification of novel drug targets. In this study, we focused on the peptidoglycan (PG) layer, a rigid polymer that maintains cell size and shape. Biosynthesis of PG is catalysed by numerous enzymes including penicillin binding proteins (PBPs). DD-carboxypeptidases (DD-CPases) are low molecular weight PBPs that regulate the level of cross linking in PG. MSMEG_6113 (dacB), one of four DD-CPases in Mycobacterium smegmatis, could not be deleted by allelic exchange and consequently, was predicted to be essential for the growth of mycobacteria in vitro. Essentiality was confirmed by deleting the chromosomal copy of $d a c B$ in the presence of additional copy of the gene operon at a different site. Moreover, a dacB depletion strain was constructed to assess the resultant phenotypic changes. Using scanning electron microscopy and fluorescently-labelled tags, we showed that DacB repression resulted in the shortening of cells as well as the loss of polar growth at one side of the cell. Collectively, we have proven that $d a c B$ is essential for growth of mycobacteria and as such have identified it as a novel target for TB drug development.

Key words: Mycobacterium smegmatis, DacB

\section{ID-P-27: HIGH CO-OCCURRING STI AND HIV PREVALENCE AND THE POOR TREATMENT CASCADE AMONG FEMALE SEX WORKERS IN DURBAN, SOUTH AFRICA.}

Letitia Greener', Ross Greener', Mags Beksinska', Kedibone Sithole', Yves Lafort ${ }^{2}$, Jennifer A Smit ${ }^{1}$

'School of Clinical Medicine, Department of Obstetrics and Gynaecology, University of the Witwatersrand, MatCH Research Unit (MRU), South Africa, ${ }^{2}$ Ghent University

National studies in South Africa have estimated HIV prevalence among Female Sex Workers (FSWs) to be between $46-69 \%$. In high prevalence settings or with key populations the World Health Organization recommends regular HIV testing, with linkage to care, however, information exploring FSWS HIV or STI testing behaviour or linkage to care is scarce. The data reported in this paper were collected in 2012 using a cross sectional survey and HIV rapid testing in Durban, South Africa. Participants were recruited using respondent driven sampling, total sample was 400 FSWs. Participants were between 21-30 years of age; and estimated to have between 1-7 partners a week. Few FSWs knew the HIV status of their regular paying partners $(12.2 \%)$ or their last non-paying partner (8.3\%). Condom use at last sex with a paying partner was estimated at $71 \%$ and lower with regular nonpaying partners (63\%). Additionally $65.3 \%$ of FSWs reported condom breakages in the last year. The biological data collected indicate that HIV prevalence among FSW in Durban is $66.9 \%$. Of the FSWs who self-reported an HIV positive status $18 \%$ were currently accessing antiretroviral therapy. Seventy percent of FSWs reported experiencing an abnormal discharge or genital ulcer within the last 12 months. There are multiple risk factors that increase FSWs risk of STI and HIV infection and transmission. These findings highlight the need and importance of integrating, providing and promoting family planning in addition to regular STI and HIV prevention, testing and treatment services for FSWs.

Key words: Female sex workers, sexually transmitted infection, HIV testing

\section{ID-P-28: COMPARATIVE RESULTS OF A NOVEL FLOW CYTOMETRIC ASSAY (FA) FOR EARLY DETECTION OF CRYPTO- COCCAL ANTIGEN (CRAG) AGAINST LFA AND EIA IN HIV-INFECTED PATIENTS WITH A CD4 COUNT <100CELLS/ML}

Lindi Coetzee', Keshendree Moodley², Debbie Glencross ${ }^{3}$

'School of Pathology, Department of Molecular Medicine and Haematology, University of the Witwatersrand, ${ }^{2}$ Wits Health Consortium, ${ }^{3}$ Wits Medical School

Reflexed cryptococcal antigen (CrAg) testing on CD4 samples is indicated for early detection of cryptococcal disease in $\mathrm{HIV}+$ patients with CD4 counts $<100$ cells/ 
ul. A CrAg flow cytometric assay (FA, Immy, USA) was developed and performance evaluated against a lateral flow assay (LFA) and two CrAg enzyme immunoassays (EIA). Sixty-three remnant EDTA CD4 samples, with confirmed LFA CrAg results were analysed by FA. Mean fluorescence intensity (MFI) was reported, with preestablished positive and negative cutoffs. Plasma from the same samples were re-tested by automated EIA ('Alpha' CrAg EIA, Immy, US and 'Premier' CrAg EIA kits), on the ThunderBolt platform (Gold Standard Diagnostics, USA). EIA results were reported as positive:negative according to optic density values with preset limits of detection. Sensitivity and specificity calculations were performed. A validation panel ( $n=20$ spiked negative plasma samples) was assessed for assay accuracy. LFA reported 21 negative vs. 42 positive. The negative:positive test results for EIA assays were 37: 26 (Immy EIA), 33:30 (Premier EIA) and $31: 32$ with the FA. Sensitivity of the FA was $86 \%$ versus Immy-EIA; $94 \%$ versus Premier-EIA and $76 \%$ against LFA (7/26 positive LFA samples tested negative with EIA and FA tests). In total, 56/63 samples showed concordance across both EIA and flow assays (89\%). Specificity of the FA was $100 \%$ against both LFA and Immy-EIA and $90 \%$ against Premier EIA. The validation panel yielded $100 \%$ accuracy across all assays. The FA showed acceptable sensitivity and specificity, making it suitable for integration into routine CD4 testing.

Key words: Flow cytometry, Cryptococcal antigen

\section{ID-P-29: COMPARISON OF THE NEW FULLY AUTOMATED VOLUMETRIC AQUIOS FLOW CYTOMETER PANLEUCOGATE (PLG) PLATFORM FOR CD4-T LYMPHOCYTE ENUMERATION TO EXISTING PREDICATE PLG TECHNOLOGY IN SOUTH AFRICA.}

\author{
Lindi Coetzee', Sherry Drury², Debbie Glencross ${ }^{3}$
}

'School of Pathology, Department of Molecular Medicine and Haematology, University of the Witwatersrand, ${ }^{2} \mathrm{NHLS},{ }^{3}$ Wits Medical School

The National Health Laboratory Service (NHLS) offers CD4 T-cell enumeration for the HIV programme using semi- or fully automated flow cytometers/preparation systems, with PanLeucogated (PLG) methodology. The new PLG/ Aquios "load and go" cytometer (Beckman Coulter) was validated against the predicate automated MPL/CellMek platform. Remnant EDTA blood samples(M121020, WITS Ethics Clearance) were analysed on the reference platform and retested on three Aquios systems according to manufacturer specifications. Data was analysed using GraphPad software for general statistics and BlandAltman analysis with $95 \%$ limit of agreement (LOA). The percentage similarity model (\%SIM) assessed agreement of absolute CD4 counts (CD4\#) and CD4 percentage of lymphocytes (CD4\%) between platforms, with a $\%$ coefficient of variance (\%CV) indicating accuracy. CD4 reference counts ( $n=1885)$ varied between 1-3469cells/ $\mu \mathrm{l}$, with a mean of $397 \mathrm{cells} / \mu \mathrm{l}$. \%SIM agreement of $95.56 \pm 11.53 \%$ (\%CV of $12.2 \%$ ) for CD4\# and $99.4 \pm 83$ (\%CV of $8.4 \%$ ) for CD4\% was noted. \%SIM values corrected for values $<100 \mathrm{cells} / \mu \mathrm{l}$ resulted in $95.1 \pm 8.16$ (\%CV of $8.58 \%$ ) for CD4\#, while retaining tight results for CD4\% (99\%). Bland-Altman analysis confirmed the $5 \%$ under-estimation

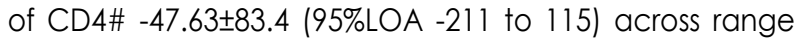
of CD4 values. The new Aquios/PLG platform showed excellent correlation with the reference platform for both CD4\# and CD4\%. Daily quality control data for this period confirmed good reproducibility and stability over time with \%CV's<8\% for both parameters. PLG/Aquios was deemed suitable to replace outdated XL cytometers in NHLS CD4 laboratories

Key words: CD4 enumeration, Aquios, flow cytometry

\section{ID-P-30: USE AND FIT OF FILTERING FACEPIECE RESPIRATORS IN A DEPARTMENT OF ANAESTHESIOLOGY}

Marthinet Nlemandt, Helen Perrie, Juan Scribante, Analee Milner. Department of Anaesthesiology, School of Clinical Medicine, Faculty of Health Science, University of the Witwatersrand.

The most reliable protection against nosocomial tuberculosis transmission in theatre is the consistent and correct use of filtering facepiece respirators (FFRs) with an N95 filter as recommended by the Centre for Disease Control and Prevention. The objectives of this study were to describe the anaesthetists' compliance and comfort with the use of FFRs, their donning technique and the outcome of a fit test. A two part study with prospective, contextual, descriptive design was done. For Part 1 a self-administered survey was distributed to anaesthetists selected by convenience sampling ( $n=140$ ) from the Department of Anaesthesiology, affiliated to the University of the Witwatersrand. Part 2 was a pilot study done in the theatre complex of Charlotte Maxeke Johannesburg Academic Hospital with members of the department selected by stratified random sampling ( 10 male and 10 female). In Part 2 the donning technique was directly observed, then corrected and followed by qualitative respirator fit tests. The results showed that compliance with the use of the FFR was inadequate, scoring 14,5 out of 25. The FFR is deemed uncomfortable, with a "discomfort score" of 9,9 out of 21 . Of the 20 anaesthetists in Part 2, only one achieved $100 \%$ for donning technique and only six (30\%), five males and one female, passed the fit test. In conclusion the poor compliance and discomfort of FFR use should be addressed. Formal training on FFR donning is required. Relying on only one size and model of FFR at this hospital is inadequate.

Key words: Tuberculosis, respiratory protection, anaesthetists 


\section{ID-P-31: LOCALIZATION OF MYCOBACTERIUM SMEGMATIS DD- CPASES AND OTHER CELL WALL REMODELLING ENZYMES}

Masethabela Maphatsoe', Christopher Ealand², Bavesh Kana² 'School of Pathology, University of the Witwatersrand, Centre of Excellence for Biomedical TB Research, ${ }^{2}$ DST-NRF Centre of Excellence for Biomedical TB Research, School of Pathology, Faculty of Health Sciences, University of the Witwatersrand

Mycobacterium tuberculosis (Mtb), the causative agent of tuberculosis (TB) remains the leading cause of death worldwide due to its complex mycobacterial cell envelope which confers substantive tolerance to antibiotics. In this regard, the design of effective antiTB drugs requires an enhanced understanding of the processes associated with bacterial cell assembly and cell division. DD-carboxypeptidases (DD-Cpases), classified as low molecular weight penicillin binding proteins (LMW PBPs) have been implicated in the remodelling of the peptidoglycan (PG) and are important for cell growth and division. Remodelling of $P G$, component of the mycobacterial cell envelope requires tight spatial and temporal regulation to maintain cell wall integrity and cell size/shape. In this study we assessed localization patterns of the essential DD-CPase, DacB, in Mycobacterium smegmatis, a non-pathogenic relative of $M+b$. For this, we constructed a derivative of DacB that carried a $C$-terminal green fluorescence protein tag to determine its cellular localization. We have also localized other cell division proteins, namely DivIVA, FtsZ, and PonA2 to study their localization relative to DacB in wild type M. smegmatis and mutants defective for DD-Cpases. We demonstrate unique localization patterns for these proteins that point to an essential role in remodelling of the cell surface during division. FtsZ localized to mid-cell, where cell division is initiated while DivIVA, a division site selection protein localized to both poles. This study has contributed to the understanding of $P G$ remodelling in mycobacteria and has uncovered new drug targets for effective TB treatment.

Key words: Mycobacteria, Penicillin binding proteins, Peptidoglycan, PG remodelling

\section{ID-P-32: THE DETECTION AND QUANTIFICATION OF DIFFERENTIALLY CULTURABLE TB BACILLI (DCTB) IN PATIENTS WITH ACTIVE TUBERCULOSIS}

Melissa D. Chengalroyen', Germar M. Beukes', Bhavna G. Gordhan', Gavin Churchyard'2, Richard Hafner ${ }^{3}$, Kennedy Otwombe ${ }^{4}$, Neil Martinson ${ }^{4}$, Bavesh D. Kana

'DST/NRF Centre of Excellence for Biomedical TB Research, School of Pathology, Faculty of Health Sciences, University of the Witwatersrand, National Health Laboratory Service, ${ }^{2}$ The Aurum Institute, ${ }^{3}$ Tuberculosis Clinical Research Team, Division of AIDS, NIAID, NIH, DHHS, 4Perinatal HIV Research Unit, Faculty of Health Sciences, University of the Witwatersrand, Johannesburg, South Africa
M. tuberculosis, the causative agent of tuberculosis (TB) enters a dormant state allowing it to evade the host's immune response and reactivate years after primary infection. Resuscitation promoting factors (Rpfs) have been implicated in the reawakening of dormant bacteria and are postulated to be important for these processes. In this study we have developed an assay to resuscitate dormant $M$. tuberculosis cells and quantitatively compare them to the actively growing population. We also explore the extent of host immunity as a cue for inducing bacterial dormancy. Sputum was obtained from 110 patients with drug sensitive pulmonary TB, before the initiation of antibiotic treatment. Growth stimulation was monitored using the most probable number assay, conducted in media supplemented with culture filtrate (CF) (containing Rpfs) and Rpf-deficient culture filtrate. Standard plate counts were used to measure the culturable population. In our cohort, $19.1 \%$ harboured CF-dependent DCTB. Furthermore, $11.8 \%$ yielded Rpf-independent DCTB with the majority, $53.6 \%$ displaying both CF-dependent and Rpf-independent DCTB. The remaining $1.8 \%$ carried CF-independent DCTB and $13.6 \%$ had no DCTB. Of particular interest in this study was the use of Rpf and nonRpf containing culture filtrate to facilitate the detection of mixed strain infections by enhancing the growth of physiologically distinct strains. Our findings indicate that most individuals within our cohort harbour both Rpfdependent/Rpf-independent DCTB, suggestive of great complexity in bacterial populations during TB infection. The observation of physiologically diverse M. tuberculosis populations has important implications for treatment response and disease outcome.

Key words: Tuberculosis, sputum, resuscitation promoting factor

\section{ID-P-33: NON-VACCINE PNEUMOCOCCAL SEROTYPES IN ADULTS AGED $\geq 25$ YEARS PRE- AND POST-PNEUMOCOCCAL CONJUGATE VACCINE INTRODUCTION IN SOUTH AFRICA}

Mignon du Plessis ${ }^{1,2}$, Linda de Gouveia', Mushal Allam?, Kedibone M. Ndlangisa ${ }^{1,2}$, Nicole Wolter ${ }^{1,2}$, Cheryl Cohen ${ }^{1,2}$, Susan Meiring ${ }^{1}$, Rebecca A. Gladstone ${ }^{3}$, Stephen D. Bentley ${ }^{3}$, Keith P. Klugman ${ }^{2,4}$, Robert F. Breiman ${ }^{5}$, Lesley McGee ${ }^{6}$, Anne von Gottberg ${ }^{1,2}$

'National Institute for Communicable Diseases, National Health Laboratory Service, Johannesburg, South Africa, 2Faculty of Health Sciences, University of the Witwatersrand, Johannesburg,

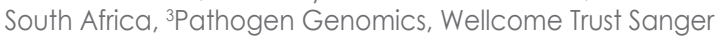
Institute, Hinxton, United Kingdom, ${ }^{4}$ Hubert Department of Global Health, Rollins School of Public Health, Department of Infectious Diseases, School of Medicine, Emory University, Atlanta, GA, USA, ${ }^{5}$ Emory Global Health Institute, Emory University, Atlanta, GA USA, 'Streptococcus Laboratory, Centers for Disease Control and Prevention, Atlanta, GA, USA

The 7-valent pneumococcal conjugate vaccine (PCV) was introduced in 2009 and replaced by PCV13 in 2011 
with subsequent declines in vaccine-serotype, invasive pneumococcal disease (IPD) in all ages. Post PCV, clonal expansion of lineages associated with antimicrobial resistance has been reported in some settings. We describe non-vaccine serotypes in adults aged $\geq 25$ years. We reviewed IPD cases, including antimicrobial susceptibility and available MLST data, reported through national, laboratory-based surveillance from 2005-2008 (pre PCV) and 2012-2015 (post PCV). Average incidence was calculated for suspected replacement serotypes for the two periods, using national census data from Statistics SA. Overall, IPD incidence in adults declined 33\% [95\% confidence interval (CI), 31\%-36\%] from 28 to 19/100,000 population. Serotype 15A increased by $64 \%(\mathrm{Cl}, 36 \%-$ $81 \%$ ) from 0.06 to $0.18 / 100,000$ population ( $p<0.001$ ). Serotype $12 \mathrm{~F}$ increased by $40 \%(\mathrm{Cl}, 20 \%-56 \%)$ from 0.31 to $0.51 / 100,000$ population ( $p<0.001$ ). Among serotype 15A, non-susceptibility to penicillin increased from 5\% (3/58) to $57 \%(110 / 194) \quad(p<0.001)$ and multidrug resistance to penicillin, tetracycline, erythromycin and clindamycin increased from $3 \%(2 / 58)$ to $35 \%(67 / 194)$ ( $p<0.001)$. Nonsusceptibility to chloramphenicol and tetracycline in serotype 12 Fincreased from $36 \%$ (107/286) to 45\% (245/561) $(p=0.03)$. Clonal complexes 63 and 989 were identified among serotypes 15A (1/2) and 12F (23/37), respectively. While childhood pneumococcal immunization has yielded declines in IPD in adults, potential replacement due to antimicrobial non-susceptible serotypes $12 \mathrm{~F}$ and 15A was noted. Globally-disseminated PMEN clone Sweden15AST63 was identified.

Key words: Pneumococcus, Vaccine, Non-PCV Serotypes, MLST

\section{ID-P-34: OBSERVING THE SAFETY AND RISK OF FUNERAL INDUSTRY PRACTICES IN SOUTH AFRICA: CURBING THE SPREAD OF INFECTIOUS DISEASES}

\footnotetext{
Minja Milovanovic 1,2, Limakatso Lebina², Daphney Maphakula²,
} Floyd Makete ${ }^{2}$, Neil Martinson ${ }^{2,3}$

ischool of Clinical Medicine, Faculty of Health Sciences University of the Witwatersrand, 2Perinatal HIV Research Unit, Faculty of Health Sciences, University of the Witwatersrand, Johannesburg, South Africa, ${ }^{3}$ Centre for Tuberculosis Research, Johns Hopkins University School of Medicine, Baltimore MD, USA, SOMCHAT MRC Collaborating Centre for HIV/AIDS and TB, South Africa

Personnel working in funeral homes are at risk of being exposed to infectious hazards like: Tuberculosis, MRSA, Hepatitis B/C, HIV and Ebola. By collecting preliminary observational information on the risky handling practices and equipment used in funeral homes, this study hopes to provide insight into how possible infectious disease could be spread through unprotected practices and lack of safety equipment. A cross-sectional study on facility assessments, qualitative interviews and process mapping were conducted in funeral homes in Soweto, Gauteng and Klerksdorp Northwest, South Africa. We report interim results of 20 facility assessments, 10 process mappings, 26 funeral home personnel interviews and 5 family interviews. Facility assessments indicated that most of the funeral homes had the minimum required safety equipment however during the process mapping and observations some of the equipment was not used; $63 \%$ had boots in stock but none were used during observations. Gloves, aprons and face masks were the most utilised equipment but no guidelines were available on wearing and removal. During observations personnel came in contact with the body and 2/10 (20\%) were exposed to blood during the body preparation. Some of the facilities did not have separate disposal for hazardous wastes: disposed in normal municipal bins, burnt or buried. In conclusion, we observed a lack of standardisation on safety equipment in the funeral homes which increases the funeral home personnel risk of exposure to hazardous and infectious materials. Furthermore, the observed lack of appropriate waste disposal procedures could pose a risk to the community.

Key words: Infectious disease, funeral homes, safety equipment, South Africa

\section{ID-P-35: FUNCTIONAL ANALYSIS OF THE CYDDC ENCODED TYPE ABC TRANSPORTER IN MYCOBACTERIUM SMEGMATIS}

Moeketsi Moseki, Bavesh Kana

School of Pathology, University of the Witwatersrand, Center of Excellence for Biomedical TB research

Mycobacterium tuberculosis (Mtb), the etiologic agent of tuberculosis (TB), has exerted an enormous death toll on the human race. During infection, Mtb survives in a poorly described persistent state, within a complex inflammatory lesion where it is protected from immune assault. Currently, TB treatment is complicated by the emergence of drug resistant strains and co-infection with HIV. To address this, our work focuses on the electron transport chain (ETC) of mycobacterial species, a tractable area of mycobacterial metabolism for drug development. Herein, we study the cydDC-encoded ABC transporter, which is required for the assembly of cytochrome bd oxidase (CbdO), a respiratory enzyme that provides for energetic requirements under hypoxia. Using an E. coli cydD null mutant, we tested whether the loss of a spectroscopic signature associated with a functional $\mathrm{CbdO}$ could be restored by heterologous expression of the Mtb cydD gene. Heterologous expression complemented the E.coli CbdO-defective mutant, evidenced by the presence of heme $\mathrm{d}$ which is diagnostic for a functional $\mathrm{CbdO}$. The complemented strain was exposed to hydrogen peroxide 
to induce oxidative stress and displayed tolerance relative to the $c y d D$ null mutant. Furthermore, we also assessed mycobacterial strains defective in the main aerobic branch for tolerance to oxidative stress and found increased sensitivity to oxidative damage. Collectively, these data uncover new vulnerabilities in the components of the ETC that can be exploited for development of new drugs for treating both drug resistant and susceptible TB.

KeyWords: TB, Cytochrome bd Oxidase, cydDC, oxidative stress

\section{ID-P-36: MONITORING INSECTICIDE RESISTANCE IN THE AN. GAMBIAE COMPLEX FROM KWAZULU-NATAL, SOUTH AFRICA}

\author{
Mohlominyana Mofokeng', Givemore Munhenga ${ }^{2}$ \\ Lizette L. Koekemoer
}

'School of Pathology, Wits Research Institute for Malaria (WRIM), Faculty of Health Sciences, University of the Witwatersrand, ${ }^{2}$ National Health Laboratory Services, Vector Control Reference Laboratory, Centre for Opportunistic, Tropical and Hospital Infections, National Institute for Communicable Diseases, Wits Research Institute for Malaria, School of Pathology, Faculty of Health Sciences, University of the Witwatersrand, Johannesburg, South Africa

Mosquitoes from the genus Anopheles are responsible for transmission of malaria, and in sub-Saharan Africa three of the four main vectors are species from the Anopheles gambiae complex, while the forth species are a member of the An. funestus group. Anopheles arabiensis is currently the main vector in countries such as South Africa, Sudan and Ethiopia. There have been many initiatives in controlling this vector which includes the use of insecticides. However, this is becoming problematic due to selection of resistance against insecticides being used. The aim of this study was to monitor An. arabiensis susceptibility to insecticides in a malaria endemic province in South Africa, KZN. Mosquitoes were sampled outdoors from

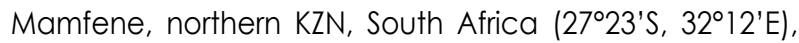
using African clay-pots and trapping plastic buckets. Molecular techniques were used to identify collected specimens to species-specific level and determining Plasmodium infectivity. In addition, susceptibility to four classes of insecticides was analysed in pooled $\mathrm{Fl}$ progeny of An. arabiensis reared from wild-caught females using the standard WHO bioassay protocol. Species analysis showed that the majority of the samples collected were An. arabiensis contributing $96 \%$ of total specimens analysed. Of the 761 specimens tested for Plasmodium falciparum infectivity none were positive. Insecticide susceptibility diagnostic tests showed resistance to DDT (77\% mean mortality) and suspected resistance to the pyrethroid deltamethrin, at a mean mortality of $95 \%$ and susceptible to both Malathion (an organophosphate) and bendiocarb (a carbamate) with a mean mortality of $100 \%$. These findings suggest that An. arabiensis population from $\mathrm{KZN}$ is resistant to DDT being used by the malaria control programme. Insecticide resistance management is important to prevent it impacting on the malaria control program.

Key words: Malaria, Anopheles arabiensis, insecticides, South Africa

\section{ID-P-37: CHEST X-RAY FINDINGS IN CHILDREN HOSPITALIZED WITH WHO DEFINED SEVERE PNEUMONIA IN A HIGH HIV PREVALENCE SETTING IN THE ERA OF BACTERIAL CONJUGATE VACCINES}

Nasreen Mahomed', David Moore², Tanusha Sewchuran², Halvani Moodley², Shabir A Madhi'

'School of Clinical Medicine, Department of Diagnostic Radiology, Respiratory and Meningeal Pathogens Research Unit, 2University of Witwatersrand

The chest $\mathrm{X}$-ray remains the commonest imaging modality for the assessment of childhood pneumonia, especially in resource limited countries. To describe and compare chest X-ray patterns in HIV-infected, HIV-exposeduninfected (HEU) and HIV-unexposed children under 5 years hospitalized with WHO-defined severe pneumonia. This study was nested within the prospective PERCH study, South African site. Children hospitalized with WHO defined severe pneumonia in Soweto, Johannesburg, were enrolled over two years (August 2011-August 2013). Chest $X$-rays were interpreted by 3 radiologists independently, blinded to all clinical data, using modified WHO chest $X$-ray interpretation criteria. Majority consensus reading was used during the data analysis phase. Interpretable chest X-rays were available in 858 (93\%) of 920 children; 9.0 months mean age. Of these 108 (13\%) were HIV-infected, 284 (33\%) were HEU, 428 (50\%) were HIV-unexposed. The commonest finding was chest X-ray primary end-point pneumonia (CXR-PEP), prevalent among $60 \%$ of HIVinfected, 33\% HEU and 38\% HIV-unexposed children. CXRPEP was twice as common in HIV-infected (OR 2.5; 95\% Cl 1.6-3.8) compared with HIV-unexposed children. Other infiltrate only was more common in HEU $(27 \%$; OR $1.4 ; 95 \%$ $\mathrm{Cl}$ 1.0-2.0) compared with HIV-unexposed children (21\%). Intrathoracic-lymphadenopathy was prevalent in 14-17\% of children and bilateral air trapping in 14-19\% of children, with no significant difference between the 3 groups. CXRPEP remains the commonest chest $X$-ray abnormality in HIV-infected, HEU and HIV-unexposed children under 5 years hospitalized for WHO-defined severe pneumonia, in the era of routine HiB and PCV immunization. HIV-infected children were twice as likely to have CXR-PEP.

Key words: Chest X-rays, primary end point pneumonia 


\section{ID-P-38: PATIENT PREFERENCES FOR SAFER CONCEPTION SERVICE DELIVERY: FINDINGS FROM A DISCRETE CHOICE ANALYSIS}

Natasha Davies ', Sheree Schwartz',2, Saiqa Mullick', Duane Blaquw $^{3}$

'School of Clinical Medicine, Wits Reproductive Health and HIV Institute (WRHI), University of the Witwatersrand, ${ }^{2} \mathrm{JHU},{ }^{3}$ Centre for Health Policy, University of the Witwatersrand

Safer-conception services (SCS) provision for HIV-affected couples trying to conceive has been recommended. However little evidence exists regarding how to best scale-up SCS. We explored patient preferences for SCS delivery attributes. Discrete choice experiment (DCE) questionnaires were conducted with patients accessing a large primary healthcare clinic in Johannesburg. Eligible individuals were $\geq 18$ years, planning pregnancy within $\leq 12$ months, and in an HIV-affected relationship. Questionnaires, informed by qualitative work, assessed how different service delivery attributes affected preferences for SCS attendance. Conditional logistic regression and partial log-likelihood comparisons were used to examine the importance of attributes on service delivery preference. From September 2015 to January 2016, 505 participants (69\% female) were enrolled. Median age was 33years [IQR:28-37]. 191 (38\%), 105 (21\%) and 209 (41\%) participants were in seroconcordant, serodiscordant and unknown status relationships respectively. The type of counselling sessions offered was the most important attribute driving SCS preferences: couples' counselling being most popular, followed by group single-sex counselling, group co-ed counselling and informational leaflets. Other attributes influenced preferences in the order: healthcare provider continuity across visits, healthcare worker type (nurse preferred over counsellor), weekend operating hours, shorter time spent at clinic and number of operating days. In sub-group analyses, women's preferences reflected the overall sample, whereas men valued counselling approach and weekend operating hours equally. Considering client preferences for private couple counselling, effective SCS implementation will require sufficient consultation time and physical space. Given persistent challenges engaging men in care, their preference for weekend services should be addressed to maximize SCS uptake.

Key words: service delivery preferences, HIV

\section{ID-P-39: DNA REPAIR AND MUTAGENESIS IN MYCOBACTERIUM SMEGMATIS}

\author{
Nelia Manamela', Bhavna Gordhan², Bavesh Kana ${ }^{2}$
}

'School of Pathology, University of the Witwatersrand, DST/NRF Centre of Excellence for Biomedical TB Research, 'University of the Witwatersrand

Upon infection Mycobacterium tuberculosis, causative agent of tuberculosis exposed to reactive oxygen and nitrogen species induced by the host immune response leading to DNA damage which results in genomic instability and eventual death of the organism. To maintain genomic stability mycobacteria use several DNA repair systems including the base excision repair(BER) pathway which entails excision of incorrect base-pairs in pro-mutagenic and cytotoxic lesions using specialised endonucleases called DNA gycosylases. Two families of DNA glycosylases in mycobacteria are the major driving forces behind base excision; formamidopyrimidine DNA glycosylases (Fpg) and endonuclease VIII (Nei) family and the endonuclease III (Nth) superfamily. Previous studies identified an antimutator role for the single Nth homologue in mycobacteria in addition to a novel interplay between the Nth and the two Nei homologues in DNA damage tolerance under oxidative stress conditions and in spontaneous mutagenesis. To understand the molecular basis of this interaction the individual effects of the Nei homologues (Neil and Nei2), together with Nth on survival and mutagenesis under UV-induced DNA damage were investigated in the current study. Defects in the BER system is expected to result in stalling of the DNA replication fork hence, the origin versus termination (Ori/Ter) ratio was measured by real time PCR in various combinatorial mutants to further delineate the role of these various DNA glycosylases during DNA repair. Collectively, our data will provide a better understanding on the collective and/ or redundant role of the two Nei DNA glycosylases in the maintenance of mycobacterial genome integrity during BER.

Key words: DNA repair and mutagenesis

\section{ID-P-40: THE LARVICIDAL AND BRINE SHRIMP ACTIVITIES OF EUGENOL AND FIVE DERIVATIVES}

Nelusha Shunmoogam-Gounden, Robyn van Zyl

School of Therapeutic Sciences, Department of Pharmacology

Division, Department of Pharmacy and Pharmacology, Wits

Research Institute for Malaria (WRIM)

Eugenol, an essential oil from the Ocimum genus of plants has been shown to exhibit biological activity against a variety of organisms, including bacteria, fungi and protozoa. Many mosquito species, including Anopheles, are vectors for pathogens and are controlled by chemical insecticides. Apart from being harmful to the environment and humans, many vectors have developed resistance to these chemicals, indicating a need for alternative vector management. In this study five eugenol derivatives: o-eugenol, eugenyl acetate, methyl eugenol, methylisoeugenol and isoeugenol were investigated for toxicological effects against Anopheles arabiensis mosquito larvae and Artemia franciscana brine shrimp nauplii. Eugenol and its derivatives were evaluated 
for their larvicidal activity against 3rd/4th instar larvae according to the World Health Organisation protocol using dichlorodiphenyltrichloroethane as a positive control. In the brine shrimp lethality assay, the nauplii were exposed to the essential oils and the positive control, potassium dichromate, for 24 hours. Methyl eugenol and methylisoeugenol displayed the greatest larvicidal activity with LC50 values of $0.0064 \pm 0.0013 \%$ and $0.0062 \pm 0.0009 \%$, respectively. Eugenyl acetate and isoeugenol showed the weakest larvicidal activity compared to eugenol and the other three derivatives, with LC50 values greater than $0.01 \%$. Isoeugenol was more selective towards the brine shrimp nauplii than the mosquito larvae with a 5.8-fold increase in toxicity (LC50 $=0.00178 \pm 0.0002 \%$ ) compared to the mosquito larvae.Interestingly, methylisoeugenol, which bears close structural similarity to isoeugenol, was the most effective eugenol derivative against Anopheles arabiensis mosquito larvae, warranting further investigation.

Key words: Eugenol, larvicidal

\section{ID-P-41: EFFICACY OF ADENO- ASSOCIATED VIRAL VECTORS EXPRESSING PRI-MIR MIMICS TO INHIBIT HEPATITIS B VIRUS REPLICATION.}

Njabulo Mnyandu', Betty Mowa', Abdullah Ely², Patrick Aburthnot

'School of Pathology, Department of Molecular Medicine and Haematology, Antiviral Gene Therapy Research Unit, University of the Witwatersrand, ${ }^{2}$ Wits \& SA-MRC Antiviral Gene Therapy Research Unit

Chronic hepatitis B virus (HBV) infection has been implicated in the development of hepatocellular carcinoma. RNA interference-mediated inhibition of HBV replication through adenoviral vectors have resulted in significant HBV knockdown. However long-term hepatocyte transduction, a pre-requisite in combating chronic infection remains elusive, owing to stimulation of the immune response that results in viral vector clearance. This study investigates the application of adenoassociated viral vectors (AAV) in the delivery of RNAi activators against HBV. DNA recombinant technology was used to construct an AAV genome bearing plasmid expressing an anti-HBV sequence (PAAVHBV) from a liver specific promoter. As a control, nano luciferase expressing plasmid (pAAVluc) was also constructed. Following luciferase assay, a significant expression of nanoluciferase from pAAVluc in a mammalian hepatocyte cell line was observed as compared to the untransfected control. Inhibition of HBV replication by PAAVHBV was tested in cells cotransfected with HBV genome bearing plasmid. Significant HBV replication inhibition (75\%) was observed. These two plasmids will then be used for AAV production and characterisation in vitro and in vivo.
With AAV's established safety profile coupled with its support of long-term expression, it is expected that longterm transduction of hepatocytes and HBV replication inhibition will be demonstrated in this study.

Key words: HBV, AAV, RNAi, micro-RNA

\section{ID-P-42: INFECTION OF THE MAIN AFRICAN MALARIA VECTORS WITH PLASMODIUM FALCIPARUM AT THE WITS RESEARCH INSTITUTE FOR MALARIA (WRIM)}

Noluthando Nkosi', Annette Bennett', Lungile Mnisi', Serena Shunmugam ${ }^{2}$, Dewaldł Engelbrecht' ${ }^{2}$, Belinda Bezuidenhout ${ }^{2}$, Maureen Coetzee ${ }^{2}$, Theresa Coetzer ${ }^{3}$, Lizette Koekemoer ${ }^{3}$

'Wits Research Institute for Malaria, School of Pathology, Faculty of the Health Sciences, University of the Witwatersrand, Johannesburg, ${ }^{2}$ Department of Molecular Medicine and Haematology, University of the Witwatersrand and National Health Laboratory Service, Johannesburg, ${ }^{3}$ Vector Control Reference Laboratory, Centre for Opportunistic, Tropical and Hospital Infections, NICD/NHLS, Johannesburg

Infection studies have been initiated at Wits Research Institute for Malaria (WRIM), with the focus on obtaining mosquito vectors infected with Plasmodium falciparum. Two Anopheles gambiae complex species were selected (Anopheles coluzzii and Anopheles arabiensis) and one member of the An. funestus group, An. funestus. These species were screened for feeding on the artificial membrane system. The aim of these infection studies is to evaluate compounds that will interact with the parasite development in the mosquito vector. Gametocytes from a Plasmodium falciparum strain (NF54-GFP-luc) expressing Green Fluorescent Protein (GFP) were cultured and fed to mosquitoes using the artificial membrane feeding system. Mosquitoes were dissected at 9-14 days post-feeding to determine oocyst prevalence on the midgut and at 14-20 days for sporozoites in the salivary glands. Oocysts were observed by fluorescence and phase contrast microscopy and counted to calculate the infection rate. Dissected salivary glands were stained with Giemsa to determine if sporozoites, which are infectious to humans, were present. The unit has achieved infection on An. coluzzii, An. arabiensis and An. funestus. GFP expression was observed and on average, more than two oocysts can generally be observed on each midgut. Hundreds of sporozoites were observed in the salivary glands. This unit has succeeded to infect important African malaria mosquitoes with the human parasite, $P$. falciparum. The Institute can now perform further research on infecting mosquitoes with the artificial membrane system.

Key words: Plasmodium falciparum, oocysts, sporozoites, fluorescence and phase-contrast microscopy 


\section{ID-P-43: MYCOBACTERIAL AMIDASES: BIOLOGICAL FUNCTION AND PUTATIVE ROLE IN CELL WALL REMODELLING}

Nombeko Sikhosana, Chengalroyen Melissa, Bavesh Kana School of Pathology, Department of Haematology, University of the Witwatersrand, Centre for Biomedical TB Research

Peptidoglycan (PG) hydrolases required for normal bacterial growth and cell division are central to the maintenance of bacterial cell wall homeostasis. Consequently, a comprehensive understanding of the remodelling of $P G$ has garnered recent interest and will aid in the development of new cell wall targeting therapeutics. Herein, we focus on a group of enzymes, the $\mathrm{N}$-acetylmuramyl-L-alanine amidases involved in PG remodelling during daughter cell separation and PG recycling. Bioinformatics analysis revealed a class of amidase_2 domain containing proteins, Ami3 whose amino acids involved in the coordination of the zinc ion are partially conserved and Ami4 whose catalytic activity remains intact. We characterized the function of these amidase_2 domain containing proteins and found that strains deficient in either of the amidases displayed defects in growth kinetics, colony morphology, biofilm formation and cell shape, an accumulation of peptides was seen as indicated by the extensive fluorescence of fluorescent vancomycin. Disruption of ami3 in particular resulted in increased susceptibility to cell wall targeting antibiotics and arrest in biofilm formation. Cellular localization studies suggested a possible interaction between Ami3 and Amil (a distinct amidase_3 domain containing protein), pointing to a possible role for Ami3 in regulating Amil catalytic activity. The data obtained establishes an essential role of Amidase_2 domain containing proteins in Mycobacterial growth, cell morphology and cell wall recycling. We therefore aim to assess the involvement of ami3 and ami4, in mycobacterial cell division, PG recycling, Amil function regulation by $\mathrm{Ami} 3$ and their role in cell-cell communication along with interaction with the extracellular matrix

Key words: Peptidoglycan recycling, novel therapeutic

\section{ID-P-44: ADDRESSING CURRENT CHOKEPOINTS IN ANOPHELES ARABIENSIS MOSQUITO MASS REARING}

Oliver Wood', Oliver Wood ${ }^{1,2}$, Givemore Munhenga ${ }^{1,2}$, Lizette Koekemoer $^{1,2}$, Basil Brooke ${ }^{1,2}$

'School of Pathology, Wits Research Institute for Malaria (WRIM), University of the Witwatersrand, ${ }^{2}$ Vector Control Reference Laboratory, National Institute for Communicable Diseases

The Sterile Insect Technique (SIT) has proved to be an effective supportive tool in the control of various insect pest species including disease vectors. With the rise of insecticide resistance, or for insect species where complete insecticidal control is difficult, this role is becoming increasingly more important. While other insect species have been controlled effectively using SIT for decades, its use for the control of malaria vectors is still in the early stages of system development. It is therefore to be expected that the first generation of equipment and techniques developed to date will still need to be further developed, refined, or even radically overhauled in the quest to effectively and efficiently produce large numbers of competitive sterile male mosquitoes for release. Through preliminary, small-scale mass-rearing mock-up operations using existing technologies, workflow and operation have been evaluated and various production chokepoints identified that need to be addressed. Following this experience, new equipment, as well as modifications to the current equipment have been designed, and are currently in the prototyping and evaluation stage. These improvements in equipment design, operation and workflow should greatly reduce the number of man-hours and staff required for mass rearing, as well as significantly reduce the cost of equipment manufacture. The success of these designs and alterations should reduce the ultimate chokepoint of overall project cost, rendering SIT more accessible.

Key words: SIT, anopheles, mass rearing

\section{ID-P-45: ENTEROVIRUS GENOTYPES DETECTED AMONG PATIENTS WITH SEVERE ACUTE RESPIRATORY ILLNESS (SARI), INFLUENZA-LIKE ILLNESS (ILI) AND ASYMPTOMATIC INDIVIDUALS IN SOUTH AFRICA, 2012-2014}

Orienka Hellferscee ${ }^{1,2}$, Florette Treurnicht2, Stefano Tempia ${ }^{2,3}$, Ebrahim Variava ${ }^{4}$, Halima Dawood ${ }^{5}$, Shabir Madhi',4, Mignon du Plessis $^{2,4}$, Cheryl Cohen ${ }^{2,4}$

'School of Pathology, Department of Medical Virology, University of the Witwatersrand, ${ }^{2} \mathrm{NICD},{ }^{3} \mathrm{CDC},{ }^{4} \mathrm{WITS},{ }^{5} \mathrm{Caprisa}$

Enteroviruses can cause severe acute respiratory infection (SARI). We aimed to characterize the enterovirus genotypes circulating among patients with SARI, influenza-like illness (ILI) and controls from June 2012 to July 2014. Syndromic SARI and ILI surveillance was performed at two sentinel sites: Klerksdorp Hospital in North West Province and Edendale Hospital in KwaZulu-Natal Province. At each site nasopharyngeal/oropharyngeal specimens were collected from SARI and ILI patients as well as asymptomatic controls. Specimens were tested for enterovirus by real-time PCR. Positive specimens were further genotyped by sequencing a region of the VP1 gene. The detection rate for enterovirus was $5.5 \%$ (87/1592), 3.2\% (103/3211), 3.1\% (46/1499) among SARI, ILI and controls, respectively. We observed a high diversity of circulating enterovirus genotypes (a total of 33 genotypes) 
from all four enterovirus species with high circulation rates for Enterovirus-B (25.9\%; 61/236) and Enterovirus-A (9.3\%; $22 / 236)$ compared to Enterovirus-C $(4.7 \% ; 11 / 236)$ and Enterovirus-D (3\%; 7/236) (p-value <0.05). Echovirus 30 (9.9\%, 10/101), Coxsackie virus B5 $(7.9 \%, 8 / 101)$, Enterovirus-D68 $(6.9 \%, 7 / 101)$ and Coxsackievirus B4 $(5.9 \%, 6 / 101)$ had the highest prevalence. There was no difference in disease severity between the different enterovirus species. We showed that there was a high diversity in the sequences of the enterovirus species that circulated in South Africa. Enteroviruses are detected in SARI and ILI patients but are also detected in asymptomatic controls, and no disease association of enterovirus species to disease severity could be determined.

Key words: Enterovirus, Pneumonia, South Africa

\section{ID-P-46: EFFECT OF EXTENDED STORAGE AT AMBIENT TEMPERATURE OF FAECAL SPECIMENS FOR GENEXPERT CLOSTRIDIUM DIFFICILE TOXIN PCR}

Phineas Makola, Karren Leroux, Desmond Schnugh

Faculty of Health Sciences, Clinical Microbiology and Infection Control

C. difficile is globally recognized as the largest cause of hospital acquired diarrhoea associated with longer hospitalizations and/or prolonged antibiotic administration. Studies have indicated an associated incidence rate of $25-55 \%$ and mortality rate of $15 \%$ with Clostridium difficile infection (CDI) in hospitalized patients. The transportation of $C$. difficile specimens from clinical/ hospital settings to the laboratory is a logistic challenge. Often samples reach the laboratory approximately 2 to 3 days after being collected. This study aims to evaluate the stability of $C$. difficile DNA in faecal specimens stored at room temperature over a 7 day period. $24 \mathrm{C}$. difficile Toxin B positive faecal specimens from various state hospitals were tested consecutively for $\mathrm{C}$. difficile at the National Health Laboratory Service (NHLS) Charlotte Maxeke Johannesburg Academic Hospital (CMJAH) Infection Control unit. The samples were tested daily for 7 days using the GeneXpert C. difficile kits (Cepheid).The crossing point (CT) values for Toxin B were compared using a oneway Anova. All samples remained positive throughout the 7 days period with no significant variations observed in their Toxin B CT values, $p=0.45$. This finding demonstrates a considerable adaptation of $C$. difficile to environmental conditions, especially room temperature. Therefore the rejection criteria of 48 hours for $C$. difficile samples can be extended to 7 days as no effect on results was observed.

Key words: intervention to control C. difficile

\section{ID-P-47: CHARACTERIZATION OF MYCOBACTERIAL PEPTIDOGLYCAN REMODELLING ENZYMES}

Poppy Mashilo, Bavesh Kana

School of Pathology, University of the Witwatersrand, DST-NRF Centre of Excellence for Biomedical TB Research

Tuberculosis (TB) is one of the leading causes of death worldwide and still remains one of the most difficult infectious diseases to treat. The emergence of new drug resistant strains of Mycobacterium tuberculosis (Mtb), the causative agent of TB has led to an urgent need for development of highly effective novel drugs that have the potential to reduce duration of treatment and eradicate TB. However, the issues surrounding the permeability of the mycobacterial cell wall has hindered the development of these drugs. This necessitated the identification of novel drug targets, one of which is the mycobacterial peptidoglycan (PG) layer, along with enzymes associated with its remodelling. $P G$ is a critical component of the cell wall and its remodelling in Mtb is speculated to be of utmost importance. Remodelling is facilitated by numerous enzymes including penicillin binding proteins (PBPs), a set of membrane-bound enzymes targeted by beta-lactam antibiotics. Other PBPs have been observed to exert their functions through interaction with various partners. This study focuses on characterization of putative interacting partners of LMW PBP, DD-carboxypeptidases (DD-Cpases) MSMEG_2433 and MSMEG_6113 in Mycobacterium smegmatis. This is accomplished by confirming interaction with putative partners using bacterial two-hybrid system and characterizing the putative Mycobacterium smegmatis DD-carboxypeptidase partners through gene knockout and localization studies. Collectively, this study will enhance our understanding on the physiological role of DD-Cpases in mycobacterial PG remodelling and provide us with clearer insight as to whether they act as individual enzymes or exert their function through interaction with partnering proteins.

Key words: Mycobacterial peptidoglycan, DD-carboxypeptidases

\section{ID-P-48: CHARACTERISATION OF A REPLICATION-COMPETENT HBV SEQUENCE FOR THE GENERATION OF A STABLE VIRUS-PRODUCING CELL LINE}

\author{
Prashika Singh, Patrick Arbuthnot, Abdullah Ely
}

School of Pathology, Department of Molecular Medicine and Haematology, Antiviral Gene Therapy Research Unit, Faculty of Health Sciences, University of the Witwatersrand

Hepatitis B virus (HBV) infection is a global health problem with an estimated 240 million chronic carriers worldwide, with sub-Saharan Africa as a region of hyper-endemicity. 
$\mathrm{HBV}$ is classified into 10 genotypes (A-J) with the various genotypes differing in disease progression and treatment efficacy. Lack of suitable infection models has hampered the understanding of the virus. The recent, generation of cell lines that are infectable by HBV have yielded an important resource for delineating the molecular biology of HBV and the development of novel therapeutic. The project aimed to generate a stable cell line for the production of infectious viral particles. A greater-thangenome-length $\mathrm{HBV}$ sequence $(1.3 \times$ subgenotype A 1$)$ was designed which would form the template for generation of the stable cell line. The sequence was amplified using PCR and convenient restrictions sites were introduced and subcloned into the TOPO® XL PCR vector. HuH7 cells were transfected with PCR-TOPO HBV Al 1.3 and measurement of markers of viral replication demonstrated this plasmid produced viral particles efficiently. Using this sequence to create a stable HBV-producing cell line will provide a constant supply of infectious viral particles that can be used for cell culture models of HBV infection.

Key words: HBV, subgenotype Al, cell lines

\section{ID-P-49: BREWER'S YEAST: A POTENT INDUCER OF SICKNESS RESPONSES AND INFLAMMATORY MEDIATORS}

Rachael Dangarembizi', Kennedy Erlwanger ${ }^{2}$, Christoph Rummel ${ }^{3}$, Joachim Roth ${ }^{3}$, Michael Madziva ${ }^{4}$, Lois Harden ${ }^{4}$ 'School of Physiology, Brain Function Research Group, ${ }^{2}$ School of Physiology, University of the Witwatersrand, ${ }^{3}$ Justus Liebig University, Giessen Germany, ${ }^{4}$ School of Physiology, University of the Witwatersrand

Brewer's yeast is extensively used as an experimental pyrogen in studies investigating the efficacy of antipyretic phytomedicines, although very little information is available on its potency or mechanisms in inducing sickness and inflammation. The aim of this study was to characterise sickness responses and molecular mechanisms associated with Brewer's yeast administration. Male Sprague Dawley rats (150-200 g) were implanted with temperature-sensitive radiotelemeters and assigned to receive a subcutaneous injection of either $20 \mathrm{ml} / \mathrm{kg}$ of $20 \%$ (w/v) Brewer's yeast or saline. Core body temperature, cage activity, food intake and body mass were recorded daily for 6 days. Blood for cytokine bioassays and brains for immunohistochemistry were collected from a separate group of rats similarly injected with Brewer's yeast or saline. Brewer's yeast induced fever (peak increase $1.2^{\circ} \mathrm{C}$; 8 h after injection) lasting for $72 \mathrm{~h}$. A marked reduction in cage activity (80\%) and food intake (70\%) was observed. Body mass similarly decreased (max 6\%) and body mass gain remained stunted for 6 days. Yeast injection increased plasma levels of bioactive tumor necrosis factor (TNF)-a and interleukin (IL)-6 as well as mRNA expression of these cytokines in the hypothalamus. Immunohistochemical staining showed activation of pro-inflammatory signaling pathways: the signal transducer of transcription (STAT)-3, nuclear factor (NF)-KB, NFIL6 and induction of cyclooxygenase (COX)-2 in endothelial cells. Our study shows that Brewer's yeast is indeed a potent inducer of sickness responses which activates a suite of inflammatory mediators and signalling pathways in both the periphery and the brain.

Key words: Brewer's yeast, fever, sickness behaviour, cytokines

\section{ID-P-50: PREDICTORS OF TIME TO SPUTUM CULTURE CONVERSION IN MDR AND XDR TB PATIENTS AT KLERKSDORP-TSHEPONG HOSPITAL}

Relebohile Ncha', Mary Kawonga ${ }^{1}$, Neil Martinson², Kennedy Otwombe², Ebrahim Variava ${ }^{3}$

'School of Public Health, Department of Community Health, ${ }^{2}$ Perinatal HIV Research Unit, University of the Witwatersrand, Tshepong Hospital

Early sputum culture conversion from positive to negative is an important step in the management of drug resistant tuberculosis (DR TB) and is associated with favourable outcomes. This study will assess the predictors of time to sputum culture conversion (TTSCC) in patients with multidrug resistant (MDR) and extensively drug resistant (XDR) TB at Tshepong Hospital. We conducted a retrospective cohort study, abstracting data from medical records of 526 MDR and 47 XDR TB patients started on TB treatment during 1 January 2012 to 31 December 2014. We abstracted data on patient characteristics (demographic, clinical and laboratory results), and dates of sputum testing and sputum culture conversion. We used summary statistics to describe patient characteristics and median time to sputum conversion, and performed survival analysis and Cox regression to determine predictors of TTSCC. Of the 574TB patients in the study: the median age was 38 years; $36 \%$ were male; 449 (78\%) were HIV infected (of these $87 \%$ had a CD4 count <350); and 374 (87\%) had a positive sputum culture on admission of whom $59 \%$ had a high sputum load. The median TTSCC was 57 days (IQR 29109). Further findings, including Predictors of TTSC will be presented. Understanding the predictors of TTSC allows for patients who are at high risk of delayed sputum conversion to be identified early so that a treatment plan can be developed to achieve favourable treatment outcomes.

Key words: MDR/XDR Sputum conversion 


\section{ID-P-51: ASSOCIATION OF FCГ RIIIB GENOTYPIC VARIANTS WITH MARKERS OF DISEASE SEVERITY IN HIV - 1 INFECTED WOMEN}

\author{
Ria Lassaumiere ${ }^{1,2}$, Glenda Gray ${ }^{3}$, Louise Kuhn ${ }^{4}$, Caroline T. \\ Tiemessen ${ }^{2}$ \\ ISchool of Pathology, Department of Virology, University of \\ the Witwatersrand, ${ }^{2}$ Centre for HIV and STIS, National Institute \\ for Communicable Diseases (NHLS), ${ }^{3}$ Perinatal HIV Research \\ Unit, Chris Hani Baragwanath Hospital, Soweto, South Africa, \\ ${ }^{4}$ Gertrude H. Sergievsky Centre, College of Physicians and \\ Surgeons, Department of Epidemiology, Mailman School of \\ Public Health, Columbia University, New York, USA
}

Cellular receptors for the Fc portion of immunoglobulin $G$ (lgG) - so called Fc gamma receptors (FcyR) - mediate several key immune effector functions that include antibody-dependent cellular cytotoxicity, phagocytosis, respiratory burst, and release of pro-inflammatory mediators. The magnitude of FcyR-mediated immune responses is affected by genotypic variants that alter the receptor's binding affinity for IgG (FcyRlla-H131R and FcyRllla-F158V), subcellular localization (FcyRllb-I232T), post-translational modification (FcyRIllb-HNAla/b/c), and surface density (gene copy number variability). In a crosssectional study, we indirectly assessed a role for FcyRmediated effector functions in modulating HIV-1 disease severity using FcyR variants as a proxy for functional capability. We genotyped $282 \mathrm{HIV}-1$ infected, treatmentnaïve South African Black women for all known functional FCYR variants and compared markers of HIV-l disease severity - plasma viral load and CD4+ T cell counts - across different genotypes. The FcyRlllb-HNAlb|lb genotype (confers reduced neutrophil mediated effector functions) significantly associated with lower viral loads compared to the HNAla|la and HNAlb|lc genotypes $(\mathrm{P}=0.038$ and $P=0.009$, respectively) and higher $C D 4+T$ cell counts compared to the HNAla l la genotype $(P=0.017)$ and all heterozygous combinations ( $\mathrm{P}<0.05$ for all comparisons). In addition, possession of more than two FCGR3B gene copies (confers enhanced neutrophil mediated effector functions) associated with higher viral loads ( $P=0.015)$, while less than two FCGR3B gene copies associated with higher $C D 4+T$ cell counts ( $P=0.042)$. In conclusion, this study describes a novel association for FcyRlllb allotypes, and by inference a potential role for neutrophils, in modifying HIV-1 disease severity.

Key words: HIV, Genetics, Fc receptors

\section{ID-P-52: MYCOBACTERIUM SPECIATION FROM FORMALIN-FIXED PARAFFIN EMBEDDED TISSUE BLOCKS}

Rindidzani Magobo ${ }^{1,2}$, Michelle McCabe ${ }^{3,1}$, Sharlene Naidoo ${ }^{3,1}$, Yvonne Perner $3^{3,1}$, Martin Hale ${ }^{3,1}$

'School of Pathology, Department of Anatomical Pathology, University of the Witwatersrand, ${ }^{2}$ Molecular Pathology

Laboratory, ${ }^{3}$ National Health Laboratory Service, Charlotte Maxeke Johannesburg Academic Hospital, University of Witwatersrand, Faculty of Health Sciences
Histological diagnosis of extrapulmonary mycobacterial infections depends on the presence of granulomatous inflammation, caseation necrosis and the presence of acid fast bacilli as demonstrated by a ZiehI Neelsen stain. Several challenges including reduced PCR yield due to crosslinking between DNA and protein, DNA fragmentation caused by formalin fixation of tissue samples are often encountered when detecting mycobacterial DNA from formalin-fixed paraffin embedded (FFPE) tissue blocks. To address this issue, a molecular test that is able to amplify small DNA fragments is necessary. Therefore, this study sought to evaluate the performance and the impact of MYCODIRECT 1.7 LCD array for the detection and speciation of mycobacteria from FFPE tissue blocks in a routine diagnostic histopathology laboratory setting. A total of 70 samples were used, of which 60 samples were from FFPE tissue blocks: 30 Mycobacterium positive samples (results obtained with in-house PCR) and 30 non-Mycobacterium pathogens. The remaining 10 were from culture positive samples used for speciation confirmation. The sensitivity of the assay was assessed by comparing the MYCODRECT 1.7 LCD Array results with those obtained using in-house PCR (Gold standard), and specificity assessed by including samples positive for pathogens other than Mycobacteria. The array yielded the detection sensitivity and specificity of $87 \%(26 / 30)$ and $100 \%(30 / 30)$ respectively. Speciation yielded a concordance rate of $90 \%(9 / 10)$. MYCODIRECT 1.7 LCD array showed high sensitivity and specificity for detection and speciation of mycobacteria; and significantly reduced turnaround time versus in-house PCR. Therefore, it is a suitable assay for routine histological diagnosis of mycobacterial infections.

Key words: Mycobacterium species, Formalin-fixed paraffin embedded tissue blocks

\section{ID-P-53: ADVENTURES IN PREP DELIVERY: PLANNING FOR CHANGE IN IMPLEMENTATION RESEARCH IN THE TAPS DEMONSTRATION PROJECT IN SOUTH AFRICA}

Robyn Eakle, Gabriela B Gomez, Rutendo Bothma, Francois Venter, Helen Rees

Wits Reproductive Health and HIV Institute (WRHI), University of the Witwatersrand

Flexibility in implementation research to keep up with changing paradigms is paramount to producing the data needed for programming. Successful efficacy trials proved that oral pre-exposure prophylaxis (PrEP) prevents HIV infection when taken consistently, and implementation research is now testing its deliverability in healthcare settings. We reflect on adaptations to the design of a demonstration project as policy, guidelines and regulatory landscape has changed in South Africa. The TAPS Demonstration Project (Treatment And Prevention for Sex workers) offers PrEP to HIV-negative and Immediate Treatment (IT) to HIV-positive female 
sex workers (FSWs) through a primary healthcare service tailored for FSWs in two urban clinics. TAPS focused on offering options outside of local guidelines, when co-formulated emtricitabine and tenofovir disoproxyl fumarate (FTC/TDF) was not yet locally approved for prevention and HIV treatment was limited to those with CD4 counts of less than 350. Its evaluation plan included collection of programmatic, behavioural, cost, and clinical data, as well as qualitative research. The project initiated services in Hillbrow in March and in Pretoria in September 2015. Eligibility of treatment initiation changed in August, and FTC/TDF was approved for PrEP in November 2015. The World Health Organization released expanded PrEP guidance in November, and revised ARV guidelines are expected in 2016. TAPS could not stay relevant without purposefully planning for flexibility. The project was designed for change, enabling us to accommodate new national guidelines when treatment initiation changed to a CD4 threshold of 500 . In consultation with the Department of Health, the project was updated recently to deliver PrEP and IT within newly-shifting clinical standards towards a nursedriven model, including the possibility of mobile delivery. Despite changes, the primary outcome for evaluation has been maintained: retention. Delivery platforms and data collection may evolve, but the primary outcomes of research need not change. The purpose of pilot or demonstration projects is to test the feasibility and uptake of new interventions in the real-world, providing data to support new policies. Designing adaptable studies while maintaining data integrity is imperative to ensure relevant implementation research is conducted and resources are not wasted.

Key words: PrEP, Implementation, HIV, Policy

\section{ID-P-54: TUBERCULOSIS OF THE BREAST: A DIAGNOSTIC CHALLENGE. CASE SERIES AND REVIEW OF THE LITERATURE.}

\section{Ross Hoffman}

School of Clinical Medicine, Department of Surgery, Faculty of Health Sciences, University of the Witwatersrand

TB of the breast is considered a rare condition. We present a series of nineteen cases diagnosed at the Chris Hani Baragwanath Academic Hospital Batho Pele Breast Clinic in 2015 and a review of the literature. We describe the spectrum of clinical presentation, examine the relationship between HIV and TB breast, analyse the commonly used diagnostic methods and establish a rational diagnostic approach. $68 \%(n=13)$ of our cases presented with a breast lump, half of which were clinically thought to be malignant. $26 \% \quad(n=5)$ presented with a breast abscess, and one with mastitis. At the time of presentation $73 \%$ of the patients were HIV positive, of which $71 \%$ were already on antiretroviral treatment. The median time before presenting to the health system was two months. A variety of specific diagnostic tests were utilised including TB microscopy, TB cultures, genotypic tests such as nested PCR and MTB/RIF assays (XperT®), FNA cytology, and histology. This study emphasises the diagnostic challenge that is posed by TB of the breast, as demonstrated by the large number of falsely negative tests. Despite its availability and cost effectiveness, the MTB/RIF assay is still an under-utilised diagnostic tool. Our series suggests that the finding of necrotising granulomatous inflammation of the breast is pathognomonic for TB; we recommend initiation of treatment based on this histological finding alone. After initiation of TB treatment and onward referral, the majority of the patients (74\%) have been lost to follow up.

Key words: Breast Tuberculosis

\section{ID-P-55: LOW SENSITIVITY OF XPERT MTB/ RIF ON ROUTINE PULMONARY SAMPLES FROM HIV-INFECTED CHILDREN IN SOWETO, SOUTH AFRICA}

Shobna Sawry', Harry Moultrie', Jeannette Wadula², Annelies Van Rie $^{3}$

'School of Clinical Medicine, Wits Reproductive Health and HIV Institute (WRHI), University of the Witwatersrand, ${ }^{2}$ Clinical Microbiology and Infectious Diseases, University of the Witwatersrand, '3Department of Epidemiology, Gillings School of Global Public Health, University of North Carolina, Chapel Hill

While the performance of Xpert MTB/RIF (Xpert) has been studied in hospitalized children under research conditions, little is known about the assay performance at routine paediatric outpatient clinics. We aimed to determine the performance of Xpert for pulmonary TB in HIV-infected children receiving care at a routine clinic. Smear microscopy, culture and Xpert were conducted on a single induced sputum or spontaneous sputum sample collected by routine clinic staff. Sensitivity and specificity was calculated using liquid culture as the gold standard. Of the 238 children (median age 5.8years), 153(64\%) were on ART for a median of $1.6 y$ years; only $44 \%$ were suppressed at time of sample collection. Seven children had a positive culture, of which only 1 was Xpert positive. The yields of smear microscopy, culture and Xpert were $0 \%, 4.4 \%$ and $0.6 \%$, respectively. Among the 238 sputum specimens, Xpert and culture results were available for 158 (66\%). The sensitivity of a single Xpert was 14.3\%(95\% Cl: 0.4-57.9) and specificity was $100 \%(95 \% \mathrm{Cl}$ : $97.6-100.0)$. At a routine paediatric outpatient HIV clinic, the yield of all mycobacteriological assays (smear microscopy, Xpert and culture) in HIV-positive children with suspected TB was very low $(<5 \%)$. Compared to culture, sensitivity of Xpert was low, but precision was poor. Novel non-sputum based diagnostic assays, preferably assays that do not depend on the detection of mycobacteria, are needed for diagnosis of TB in children presenting with TB symptoms at outpatient clinics.

Key words: HIV, TB, Paediatric, Xpert 


\section{ID-P-56: THE EFFECTS OF EXOGENOUS DIETARY STRESSANTS ON THE LIFE HISTORY AND INSECTICIDE RESISTANCE PHENOTYPE OF THE MAJOR MALARIA VECTOR ANOPHELES ARABIENSIS (DIPTERA: CULICIDAE)}

\author{
Shune Oliver ${ }^{1,2}$, Basil Brooke ${ }^{2,3}$
}

'School of Pathology, Department of Vector Control Reference Laboratory, ${ }^{2}$ Wits Research Institute for Malaria, ${ }^{3} \mathrm{NICD}$

The consumption of blood is crucial for the life cycle of mosquitoes such as the major malaria vector of Southern Africa, Anopheles arabiensis. Blood is, however, a potential source of host derived factors which may affect the biology of the mosquito. Malaria patients are often hyperinsulinaemic. Insulin Growth Factor (IGF) and Transforming Growth Factor (TGF- $\beta$ ) are two human derived factors that have been demonstrated to alter signal transduction in various haematophagous insects. Therefore, this study aimed to examine the effects of insulin, IGF and TGF- $\beta$ on adult mosquito longevity and insecticide resistance expression in two laboratory strains of An. arabiensis. SENN, an insecticide susceptible strain, and SENN DDT, an insecticide resistant strain, were fed insulin, IGF or TGF- $\beta$ supplemented sucrose following which the effects that these proteins have on adult longevity and insecticide resistance expression were examined. Insulin significantly reduced longevity in SENN but not SENN DDT. The biological effects of insulin were due to its' activity as a hormone, as denatured insulin did not induce the same effects. IGF and TGF- $\beta$ did not have a significant effect on the longevity of adults of either strain. Insulin augmented insecticide resistance in young SENN DDT mosquitoes, but this effect diminished with age. IGF and TGF- $\beta$ consumption significantly reduced organophosphate and pyrethroid resistance in young SENN DDT mosquitoes - an effect likely mediated by reduced detoxification and oxidative stress enzyme activity. The reduction of activity was more marked in SENN DDT. This is possibly due to increased cytokine activity in the midgut. This study demonstrates that human proteins can alter epidemiologically significant life history traits.

Key words: Insulin, IGF, TGF, resistance

\section{ID-P-57: FEASIBILITY, ACCEPTABILITY AND SOCIAL CONSEQUENCES OF CASH TRANSFERS FOR HIV PREVENTION IN ADOLESCENTS IN INNER-CITY JOHANNESBURG, SOUTH AFRICA}

Sinead Delany-Moretlwe', Pedro T Pisa' , Mphatso Kamndaya', Lucy Mashimbye', Harry Moultrie', Heena Brahmbhatt ${ }^{1,2}$

'Wits Reproductive Health and HIV Institute (WRHI), University of the Witwatersrand, Johannesburg, South Africa, 2 Johns Hopkins Bloomberg, School of Public Health, Baltimore, United States

We conducted a pilot to determine the feasibility, acceptability and social consequences of providing cash transfers (CTs) to adolescents living in a disadvantaged urban environment. We enrolled 120 consenting participants aged $\geq$ years, living in inner-city Johannesburg, who identified themselves as being sexually active and willing to obtain parental consent. Adolescents were randomised to one of three CT strategies (Unconditioned monthly payments [group A] vs. monthly payments conditioned on school attendance [group B] vs. a single direct payment conditioned on a clinic visit [group C]), with 40 participants in each group. Socio-demographic, health-seeking, school attendance, sexual behaviour and other risk outcomes were assessed at randomisation and after 6 months of intervention. Overall, $95 \%$ of participants received all planned payments (100\% group A vs. $98 \%$ group B vs. $87 \%$ group C). At baseline, most participants were male $(60 \%)$, with a mean age of 17 years. At the 6 month follow-up visit, a higher proportion of participants receiving the single direct $\mathrm{CT}$ had completed a clinic visit compared to either the unconditioned or conditioned monthly CT $(64 \%$ vs. $26 \%$ vs. $24 \%)$. While no negative impacts on relationships, alcohol, cigarette and drug consumption were reported; a significantly higher proportion of participants in the school conditioned CT group missed> 3 days of school compared to the other groups. No differences in sexual behaviour by study group were observed whilst sexual activity decreased over the intervention period in all groups. Findings demonstrate the potential of cash incentives to promote positive health seeking behaviour in adolescents.

Key words: Cash transfers, Adolescents, HIV prevention, South Africa

\section{ID-P-58: NATURAL ACQUIRED GROUP B STREPTOCOCCUS CAPSULAR POLYSACCHARIDE AND PROTEIN ANTIBODIES IN HIV-INFECTED AND HIV- UNINFECTED CHILDREN}

Sonwabile Dzanibe ${ }^{1,2}$, Peter Vincent Adrian ${ }^{1,2}$, Sheila Zena Kimaro-Mlacha ${ }^{1,2}$, Shabir Ahmed Madhi, ${ }^{1,2,3}$

'Department of Science and Technology/National Research Foundation: Vaccine Preventable Diseases, University of the Witwatersrand, Johannesburg, South Africa, ${ }^{2}$ MRC, Respiratory and Meningeal Pathogens Research Unit, University of the Witwatersrand, Johannesburg, South Africa, ${ }^{3}$ National Institutes for Communicable Diseases, Johannesburg, South Africa

Group B Streptococcus (GBS) is a major cause of invasive disease in young infants and also in older immunocompromized individuals, including HIV-infected persons, possibly due to waning of natural immunity. We compared naturally acquired antibody titres to GBS polysaccharide and surface protein antigens in HIVuninfected and HIV-infected children aged 5-7 years. A multiplex Luminex immunoassay was used to measure IgG titres against GBS capsular polysaccharides (CPS) for serotypes la, lb, III and V; and also extracellular localizing proteins which included cell-wall anchored proteins: 
fibrinogen binding surface antigen (FbsA), bacterial immunogenic adhesin (BibA), surface immunogenic protein (Sip), gbs0393, gbs1356, gbs1539, gbs0392; and lipoproteins gbs0233, gbs2106 and Foldase PsrA. HIVinfected children $(n=68)$ had significantly lower IgG GMT against CPS of serotype $\mathrm{lb}$ and $\mathrm{V}$ compared to HIVuninfected ( $n=77)$ children ( $p<0.02)$. IgG GMT against FbsA, gbs1539, gbs1356, gbs0392, gbs0393 and Foldase PsrA were however significantly higher in HIV-infected children ( $p<0.003)$, whilst titres against gbs2106 and Sip were significantly lower in HIV-infected children ( $\mathrm{p}$ $<0.002$ ). Amongst HIV infected children, IgG GMT to GBS surface proteins were higher in those with $\mathrm{CD}^{+}$ lymphocyte counts $<500$ cells $/ \mu \mathrm{L}$ compared to those who had CD4 $4^{+}$lymphocyte count $\geq 500$ cells/ $\mu \mathrm{L}$ except for Sip. The increased susceptibility to invasive GBS disease in HIVinfected individuals could be due to the lower serotype specific capsular antibody and possibly due to lower antibody to some of the GBS proteins such as Sip and gbs2106.

Key Words: group B streptococcus, proteins, capsular polysaccharides and HIV

\section{ID-P-59: AIDS/TB MORTALITY RISK AMONG RETURN MIGRANTS IN THE ERA OF ANTIRETROVIRAL TREATMENT IN RURAL SOUTH AFRICA, 2001-2014}

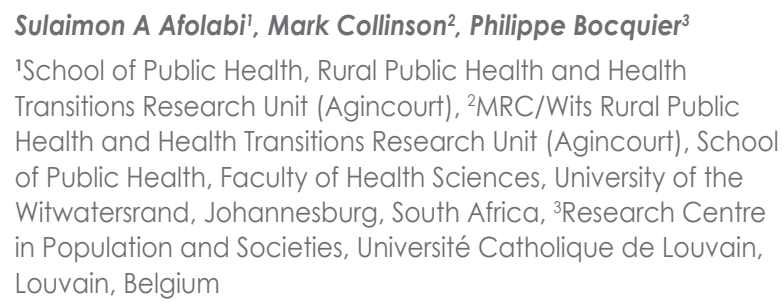

It has been established that labour migrants return back to their rural place of origin when they are ill to receive care and eventually die with AIDs/TB often being the cause. With the availability of antiretroviral treatment (ART), the mortality risk is expected to change. Hence, this study investigates the risk of AIDS/TB Mortality among return migrants before and after the availability of ART in rural South Africa between 2000and 2014. This is a cohort study of return migrants achieved by analysing health and demographic surveillance data collected between 2000 and 2014 in rural area of South Africa (Agincourt). We employed Gray and Fine model, an event history analysis technique that is appropriate when competing risk events are present as return migrants can die of other causes that can preclude their dying of AIDS/TB. The results show that return migrants have significantly higher risk of dying of AIDS/TB in comparison with their non-migrant counterparts even whether other causes of mortality are present with the evidence of it coming down in the period when ART becomes available to the general public. Migration has played a role in the AIDS/TB epidemic with increasing then decreasing contributions due to the availability of ART and other population factors. Therefore, taking migration into account is relevant not only for AIDS/TB Therefore, taking migration into account is relevant not only for AIDS/TB sentence not complete

Key words: Migration, Mortaility, AIDS, Event

\section{ID-P-60: NEGOTIATING CONDOM USE: SEX WORK AND HIV IN SOWETO}

\section{Susann Huschke}

School of Public Health, Centre for Health Policy, University of the Witwatersrand

In this paper, I use qualitative data from my on-going ethnographic study with sex workers in Soweto to discuss the negotiation of condom use between sex workers and their clients. Despite high HIV rates, condom use is not persistent in the local sex industry. My analysis offers three main reasons for this: first and foremost, sex workers in Soweto face enormous economic pressures - many are the breadwinners for their families. Sex without a condom often pays better because many clients prefer unprotected sex and are willing to pay extra for it, thereby creating an incentive for economically desperate sex workers. Second, insisting on condom use may trigger a violent response from the client, leading the sex worker to agree to sex without a condom out of fear. In other cases, clients forcefully remove the condom during sex, despite previous agreements between the sex worker and client to use condoms. Third, sex workers may run out of condoms due to the fact that carrying around large quantities of condoms has been used by the police to arrest sex workers and charge them with prostitution-related offenses under the current law, and is thus avoided by some sex workers. In the discussion, I address the underlying social and political factors that create the environment in which sex workers are in a precarious position when negotiating condom use, namely the gendered aspects of poverty in Soweto, and the effects of heteronormativity and the 'whore stigma' on the relationship between sex workers and clients.

Key words: Sex Work, HIV, Condom Use

\section{ID-P-61: MOLECULAR BASIS OF THE INTERPLAY BETWEEN THE NTH AND NEI DNA GLYCOSYLASES OF THE BASE EXCISION REPAIR PATHWAY IN MYCOBACTERIUM SMEGMATIS}

Tebogo Rantsi, Bavesh Kana, Bhavna Gordhan

School of Pathology, University of the Witwatersrand, Centre of Excellence for Biomedical TB Research

During infection, Mycobacterium tuberculosis (MTB) encounters hostile conditions which results in the generation of host-derived reactive oxygen (ROS) and nitrogen 
species (RNS) as part of the immune response to control the infection. Exposure to these reactive radicals can lead to oxidative damage of DNA which ultimately destabilises the genome and introduces mutations. However, MTB is well equipped with a number of DNA repair pathways such as the base excision repair (BER) pathway, which plays a role in maintaining genome stability and survival of the pathogen. A number of DNA glycosylases are involved in the BER pathway, including formamidopyrimidine (Fpg), Endonuclease VIII (Nei) and endonuclease III (Nth), which are the initial enzymes that are responsible for recognition and excision of damaged DNA bases. It was previously demonstrated that combinatorial deletion of nth and nei in Mycobacterium smegmatis resulted in reduced survival under oxidative stress conditions with a corresponding increase in mutation rates, suggestive of interplay between these enzymes. This study aims to further investigate the molecular basis of this synergy. Double mutants were generated using homologous recombination for site specific disruption of the nth gene in mutant strains deficient in the Neil and Nei2 glycosylases respectively. Loss of the nth gene was genotypically confirmed by PCR and Southern blot analyses. Currently, spontaneous mutation rates and survival under oxidative stress conditions is being assessed in the mutant strains. It is anticipated that this work will identify the individual and/or the combinatorial interactive role of the Nei homologues with the Nth DNA glycosylase.

Key words: TB, DNA repair, DNA glycosylases

\section{ID-P-62: SOCIAL AND BEHAVIOURAL FACTORS ASSOCIATED WITH FAILING SECOND-LINE ART - RESULTS FROM A COHORT STUDY AT THE THEMBA LETHU CLINIC, JOHANNESBURG, SOUTH AFRICA}

Tembeka Sineke ${ }^{1,2}$, Denise Evans ${ }^{2,3}$, Sara Dahlberg ${ }^{4}$, Rebecca Berhanu $^{5}$, Caroline Govathson ${ }^{2,3}$, Ingrid Jonke ${ }^{5}$, Elisabet Lonnermark ${ }^{4}$, Matthew P Fox ${ }^{6}$

'School of Public Health, University of the Witwatersrand, ${ }^{2} \mathrm{Health}$ Economics and Epidemiology Research Office, ${ }^{3} S c h o o l ~ o f$ Clinical Medicine, Department of Internal Medicine, University of the Witwatersrand, ${ }^{4}$ Department of Infectious Diseases, Sahlgrenska Academy, ${ }^{5}$ Right to Care, Johannesburg, South Africa, ${ }^{6}$ School of Public Health, Department of Epidemiology, Centre for Global Health and Development, Boston University, USA

Few options exist for patients failing second-line antiretroviral therapy, particularly in resource limited settings. Programmatic approaches for treatment failure are needed. Retrospective cohort study which included HIV-positive adults with elevated viral load $\geq 400$ copies $/ \mathrm{ml}$ on second-line ART between January 2013 - September 2014 was conducted. Patients had to have completed an Adherence Counselling Questionnaire and had repeat viral load within 6 months of intensive adherence counselling. Log-binomial regression was used to evaluate association between patient characteristics and failure to resuppress viral load within 6 months of first elevated viral load on second-line ART. 298 patients had elevated viral load on second-line ART and clinical data available. 154 (52\%) had an adherence questionnaire and were included in the analysis. Of these, 78 (59.1\%) had a suppressed viral load and 54 (40.9\%) failed to suppress. Use of herbal or traditional medicine (aRR 1.59 95\% Cl 1.002.55 ) and first elevated viral load on second-line ART (>100 000 copies/ml vs. <1 000 copies/ml; RR $2.6495 \%$ Cl 1.89$3.70)$ were strongly associated with failure to resuppress. Other factors associated included missing dose in the past week, living with family and depression symptoms. We identified several factors associated with failure to suppress viral load, but study provides limited information about reasons for these connections. Our results identify areas that could be targeted for interventions to reduce the risk for treatment failure and prevent switching to expensive third-line ART. Study highlights need for in-depth interviews to better understand these factors.

Key words: HIV, ART, Resistance, Suppression

\section{ID-P-63: FREQUENCY OF PARTNER- RELATED SOCIAL HARMS AND THEIR IMPACT ON ADHERENCE TO THE DAPIVIRINE VAGINAL RING DURING THE MTNO20/ASPIRE HIV PREVENTION TRIAL}

Thesla Palanee-Phillips', Sarah Roberts², Elizabeth Montgomery ${ }^{3}$ Krishnaveni Reddy1, Elizabeth Brown ${ }^{4}$, Jared Baeten ${ }^{2}$

ISchool of Clinical Medicine, Wits Reproductive Health and HIV Institute (WRHI), 'University of Washington, ${ }^{3}$ RTI International, Wits RHI1, Fred Hutchinson Cancer Research Centre ${ }^{4}$

Studies of daily-use PrEP have reported lower adherence among women experiencing social harms (SH). Femaleinitiated methods like the dapivirine ring, are designed to give women greater agency in HIV prevention. We characterized SH that were both study and partnerrelated and their association with adherence in MTN020/ASPIRE trial. At quarterly visits, women were asked about experiences of $\mathrm{SH}$ related to study participation or ring use. Low adherence was defined by quarterly plasma dapivirine levels $95 \mathrm{pg} / \mathrm{ml}$ among those in the dapivirine ring arm. Association between SH harm and risk of low adherence was analysed using Poisson regression models with generalized estimating equations. 85 women reported 87 partner-related $\mathrm{SH}$ of a total of $91 \mathrm{SH}$, for a cumulative incidence of $4.45 \%$. Women classified only $10 \%$ as having had a major impact on quality of life. Younger age at enrolment (mean 24 vs. 27 years, $p<0.0001$ ), having a new primary partner $(p=0.039)$, and not disclosing study participation ( $p=0.006)$ or ring use $(p=0.027)$ to the primary partner were significantly associated with reporting $\mathrm{SH}$. 
Adjusting for age, study site, and time on study, women were 2.47 times more likely to have low adherence at visits with a SH reported in the past month than at visits with no $\mathrm{SH}$ reported $(95 \% \mathrm{Cl} 1.33-4.58, \mathrm{p}=0.004)$. Although relatively uncommon $(<5 \%)$, women reporting partner-related $\mathrm{SH}$ were at increased risk of low adherence. Strategies to mitigate non-adherence in the context of SH should be evaluated in future HIV prevention studies.

Key words: Intimate partner violence, social harms, adherence, HIV

\section{ID-P-64: BASELINE CHARACTERISTICS OF HIV-1 NEGATIVE WOMEN ENROLLED INTO A CLINICAL TRIAL OF DAPIVIRINE VAGINAL RING FOR HIV-1 PREVENTION}

Thesla Palanee-Phillips', Katie Schwartz², Elizabeth Brown ${ }^{3}$, Vaneshree Govender ${ }^{4}$

'School of Clinical Medicine, Wits Reproductive Health and HIV Institute (WRHI), ${ }^{2} \mathrm{FHI} 360$, ${ }^{3}$ Fred Hutchinson Cancer Research CEntre, ${ }^{4}$ Medical Research Council

Women's vulnerability to HIV-1 remains high in subSaharan Africa. Antiretroviral prophylaxis, is a promising biomedical HIV-1 prevention strategy. ASPIRE was a phase III, randomized, double-blind, placebo-controlled trial testing the safety and effectiveness of the dapivirine vaginal ring for prevention of HIV-1 infection. Initiated in August 2012, HIV-1 sero-negative women between 18-45 years were recruited from 15 trial sites in Malawi (MW), South Africa(SA), Uganda(UG), and Zimbabwe(ZIM). Eligible participants had to be sexually active, have normal serum chemistry and haematology profiles, gynaecologic examinations, not be pregnant nor planning to fall pregnant for the duration of participation. Post enrolment, participants attended monthly visits for HIV-1 counselling/ testing, HIV-1 risk-reduction care package, adherence counselling, and provision of a vaginal ring.2629 HIV-1 negative women enrolled ( screen:enrol ratio 2.1:1). Median age was 26 years with $39 \%<25$ years and $14 \%>35$ years. Overall, $100 \%$ reported having a primary partner in the 3 months prior to enrolment and $18 \%$ reported another partner. Eight percent of SA participants report being married, with $84 \%$ in $\mathrm{ZIM}, 82 \%$ in MW and $66 \%$ in UG. Forty percent report no condom use with the last sex act prior to enrolment. Chlamydia and gonorrhoeae prevalence is $14 \%$ and $4 \%$ respectively. Baseline contraceptive use was $41 \%$ injectable DMPA, $19 \%$ contraceptive implants, $14 \%$ injectable Norethisterone enanthate, $13 \%$ intrauterine devices, and $11 \%$ oral contraceptive pills. African seronegative women at risk of HIV -1 infection can be successfully enrolled into a trial of dapvirine vaginal ring for HIV-1 prevention.

Key words: HIV, STIS

\section{ID-P-65: ASSOCIATION BETWEEN RHINOVIRUS AND STREPTOCOCCUS PNEUMONIAE AMONG CASES AND CONTROLS IN THE PNEUMONIA AETIOLOGY FOR CHILD HEALTH (PERCH) STUDY: A PRELIMINARY ANALYSIS}

Vicky Lynne Baillie, Shabir A. Madhi, Peter Adrian, David P. Moore, Azwifarwi Mudau

School of Pathology, Faculty of Health Sciences, Respiratory and Meningeal Pathogens Research Unit, University of the Witwatersrand

Influenza and RSV infections are associated with increased susceptibility to pneumococcal disease. There is a paucity of data on whether rhinovirus infection predisposes children to pneumococcal disease. PERCH is a 7-country case-control study of children 1-59 months hospitalized with WHO-defined pneumonia and agefrequency matched community controls. Rhinovirus PCRpositive nasopharyngeal/oropharyngeal specimens were subtyped (A-C). High density pneumococcal infection (HDP) was defined as pneumococcal nasopharyngeal/ oropharyngeal PCR density $>6.6$ log copies $/ \mathrm{ml}$ (>4.4 if pretreated with antibiotics) or whole blood density $>2.2 \mathrm{log}$ copies/ml, which were associated with microbiologically confirmed pneumococcal pneumonia (MCPP) in PERCH. Age- and site-adjusted odds ratios (aOR) of MCPP and HDP comparing rhinovirus-positive to rhinovirus-negative cases were calculated. Rhinovirus was not associated with MCPP $(n=55 ; a O R=1.51,95 \% \mathrm{Cl} 0.68,3.39)$ or HDP $(n=632 ; a O R=0.98,95 \% \mathrm{Cl} 0.79,1.21)$ in cases. However, among controls, rhinovirus was associated with HDP $(\mathrm{aOR}=1.47,95 \% \mathrm{Cl} 1.19,1.80)$. Among 404 cases and 399 controls positive for rhinovirus, the distribution of rhinovirus subtypes were similar between cases ( $\mathrm{A}: \mathrm{B}: \mathrm{C}=48 \%: 8 \%: 44 \%$ ) and controls $(A: B: C=45 \%: 10 \%: 45 \% ; p=0.17)$. The percent with HDP was similar by subtype among rhinovirus-positive cases $(A=51 \%, B=42 \%, C=43 \% ; p=0.3)$ but among controls HDP was less common for rhinovirus- $B(10 \%$ vs. $A=25 \%$ and $C=29 \% ; p=0.05$ ). Preliminary analyses do not support an association between rhinovirus and pneumococcal infection in children hospitalized with pneumonia.

Key words: Rhinovirus, S.pneumoniae, IPD pneumonia 


\section{MOLECULAR AND COMPARATIVE BIOSCIENCES}

\section{MCB-O-1: THE REDUCED GROWTH OF GYCEROL KINASE KNOCKOUT PLASMODIUM PARASITES IS ASSOCIATED WITH THE DOWN-REGULATION OF MEMBRANE PROTEINS INVOLVED IN PARASITE INVASION.}

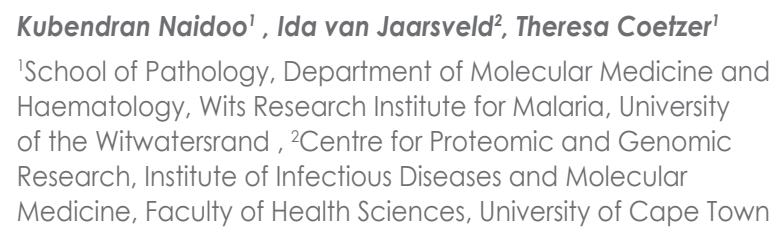

During every 48-hour asexual replication cycle within a human erythrocyte, a single Plasmodium falciparum malaria parasite can produce up to 32 progeny. This extensive proliferation implies that parasites require substantial amounts of lipids for membrane biogenesis. Plasmodium membranes are predominantly composed of phospholipids. Proteins involved in phospholipid synthesis have been explored as drug target candidates. One important protein is glycerol kinase (GK). This conserved enzyme phosphorylates glycerol to glycerol-3-phosphate, a major phospholipid precursor. We reported that $P$. falciparum parasites without the GK gene (3D7 $\triangle P F G K$ ) displayed reduced growth and a lag in development. To understand the proteins involved in phospholipid metabolism, Next Generation Sequencing and transcriptome analysis was performed to compare the gene expression profiles of wild type and 3D7 $\triangle$ PfGK bloodstage parasites. rRNA-depleted mRNA was extracted from late blood-stage parasites, where membrane biosynthesis is at its peak, and used to generate libraries which were sequenced on the Illumina HiSeq 2500 and aligned against the reference $P$. falciparum genome. Combined data from three biological replicates showed 15 upregulated and 91 down-regulated (ranging from 2-27-fold change) differentially expressed transcripts in 3D7 $\triangle P F G K$ parasites rather than aberrant expression. Membrane and invasion proteins with a glycerophospholipid component were down-regulated and suggest parasite invasion was compromised. Several uncharacterized proteins were also identified. No metabolic pathways were enriched for the differentially-expressed transcripts, but detailed analysis will provide insight into the role of GK in the development of blood-stage parasites.

Key words: Malaria, Glycerophospholipids, Transcriptome, Invasion

\section{MCB-O-2: CHARACTERIZATION OF THE MYCOBACTERIAL ELECTRON TRANSPORT CHAIN \\ Nicole Narrandes ${ }^{1,2}$, Bavesh Kana ${ }^{2}$ \\ 'School of Pathology, University of the Witwatersrand, ${ }^{2}$ DST/NRF Centre of Excellence for Biomedical TB Research}

New interventions are required to combat the current TB epidemic. Pathways involved in energy generation, such as the electron transport chain (ETC) are hypothesized to play a critical role in the survival of mycobacteria. The ETC is responsible for maintaining an energized membrane, thereby facilitating the production of energy in the form of ATP and has recently been validated as a rich source for new drug targets. We aimed to further characterize the mycobacterial ETC and its adaptations under different environmental conditions. For this, a Mycobacterium tuberculosis (Mtb) mutant strain defective for the cytochrome bd oxidase ( $\mathrm{CbO}$ ) in the ETC was analysed for in vitro growth, ATP production, susceptibility to stress, biofilm formation and ex vivo growth. While not essential for growth under carbon-rich conditions, the $\mathrm{CbO}$ is required for optimal ATP production in Mtb. In the absence of the $\mathrm{CbO}, \mathrm{Mtb}$ displayed increased sensitivity to oxidative stress but not acid stress under carbon-rich conditions. When nitrate was available as the sole nitrogen source, no difference in growth or ATP production was observed for the mutant strain, suggesting rerouting of electron flow into nitrogen metabolism. Loss of the $\mathrm{CbO}$ resulted in reduced biofilm formation, most likely the result of an inability to growth and survive under hypoxic conditions. In addition, the $\mathrm{CbO}$ deficient strain displayed attenuated growth in activated macrophages. Collectively, these data highlight the flexibility of the mycobacterial ETC under different conditions, the importance of the $\mathrm{CbO}$ for virulence and presents the $\mathrm{CbO}$ as a potential novel drug target wherein inhibition will synergize with novel emerging treatments.

Key words: TB, respiration, cytochrome oxidase 


\section{MCB-O-3: POTENCY AND BREADTH OF SINGLE CHAIN VARIABLE FRAGMENTS (SCFV) OF BROADLY NEUTRALIZING HIV ANTIBODIES}

Rebecca van Dorsten ${ }^{1,2}$, Constantinos Kurt Wibmer2, Bronwen Lambson ${ }^{2}$, Macro Weinberg ${ }^{3}$, Penny L. Moore ${ }^{2,4}$, Lynn Morris ${ }^{2,4}$

'School of Pathology, University of the Witwatersrand, ${ }^{2}$ Center for HIV and STIs, National Institute for Communicable Diseases, Johannesburg, South Africa, ${ }^{3}$ Faculty of Health Sciences,

University of the Witwatersrand, ${ }^{4}$ Center for the AIDS Programme of Research in South Africa (CAPRISA)

HIV broadly neutralizing antibodies (bNAbs) targeting the CD4 binding-site (VRC01), the V3-glycan supersite (PGT121) and the V2-apex (CAP256-VRC26.25) are being developed to prevent and treat HIV infection. Single chain antibody fragments (scFv), comprise the variable heavy and light chain domains joined in a single molecule and are therefore considerably smaller than whole lgGs. Here we explore whether scFv of three HIV bNAbs retain neutralizing activity. The variable heavy and light chain genes of VRC01, PGT121 and CAP256 were connected with a (GGGS)3 linker and cloned into a CMV-expression vector. All SCFv were expressed in HEK293F-cells and purified using a nickel column. Neutralizing activity was assessed in a TZM-bl assay against a panel of 33 viruses. Viruses with point mutations in the bNAb epitope were used to confirm the footprint of the scFv. The CAP2 56 scFv neutralized $78 \%$ of viruses compared to the lgG, although potency was reduced. The CAP256 scFv has an increased sensitivity for mutations in the V2 region (N156, T162, K169 and K171), which suggested an altered footprint. Similarly, the PGT121 scFv neutralized $68 \%$ of sensitive viruses compared to the $\lg G$, with an increased sensitivity to the N332A mutation in the V3-epitope. VRC01 scFv neutralized $100 \%$ of viruses with similar potency to the $\mathrm{lgG}$. The bNAbs VRC01, PGT121, and CAP256 can be expressed as SCFV molecules while largely retaining neutralizing activity. The loss of potency of CAP256 and PGT121 expressed as SCFvs, is likely due to the reduced affinity of scFv for the trimeric envelope glycoproteins.

Key words: HIV, scFv, bNAbs,

\section{MCB-O-4: DIVERSITY ANALYSES OF HIV-1 ENVELOPE GLYCOPROTEINS IN HIV-INFECTED INDIVIDUALS WITH AND WITHOUT BROADLY NEUTRALIZING ANTIBODIES}

Batsirai Mabvakure', Cathrine Scheepers', Bronwen Lambson' Molati Nonyane ${ }^{2}$, Salim Abdool Karim³, Carolyn Williamson ${ }^{3,4}$, Jinal Bhiman',3, Lynn Morris, Penny Moore ${ }^{1,3}$

'School of Pathology, Faculty of Health Sciences, University of the Witwatersrand, Center for HIV and STIs, National Institute for Communicable Diseases, National Health Laboratory Service, ${ }^{2}$ Center for HIV and STIs, National Institute for
Communicable Diseases, National Health Laboratory Service, Johannesburg, ${ }^{3}$ Centre for the AIDS Programme of Research in South Africa (CAPRISA), Kwa-Zulu Natal. ${ }^{3}$ Division of Medical Virology, Institute of Infectious Disease and Molecular Medicine, University of Cape Town

High levels of HIV envelope glycoprotein (Env) diversity have been associated with the development of broadly neutralizing antibodies (bNAbs). Here, we compare chronically HIV-1 infected subjects who develop bNAbs with those who did not, to assess whether lack of breadth can be attributed to low levels of viral diversity. Env nucleotide sequences were generated using Single Genome Amplification from four CAPRISA 002 cohort participants. Two participants developed neutralization breadth (CAP256 and CAP257) whereas the other two did not (CAP88 and CAP228) despite equivalently long duration of infection. Longitudinal diversity analyses were performed using Sequence Demarcation Tool (SDT). Phylogenetic analyses were performed using Bayesian Evolutionary Analysis by Sampling Trees (BEAST) software. Overall diversity increased with time in all subjects, as expected. Highest diversity was observed in CAP256 and CAP228, followed by CAP257 and least diversity in CAP88. The highest nucleotide substitution rates were observed in CAP257 (2.63 substitutions/100nucleotides/year), CAP256 (2.28 subs/100n/yr.) and CAP228 (2.07 subs/100n/yr.), and the lowest in CAP088 (0.99 subs/100n/yr.). The time to the most recent common ancestor (tMRCA) inferred from BEAST was longer than the actual time of infection for CAP256 and CAP228, suggesting the possibility of superinfection or multivariant infection. We conclude that the absence of viral diversity may limit bNAb development, as in CAP88. However, increased diversity through high mutation rates and/or recombination, while likely necessary, is not sufficient for driving the development of bNAbs.

Key words: Phylogenetics, Diversity, Virus, BEAST

\section{MCB-O-5: RESUSCITATION PROMOTING FACTORS ARE REQUIRED FOR BIOFILM FORMATION IN MYCOBACTERIUM SMEGMATIS}

Christopher Ealand, Lethabo Mashigo, Lusanda Thato Mapela Germar Beukes, Edith Erika Machowski, Melissa Dalcina Chengalroyen, Bavesh Kana

School of Pathology, Department of Molecular Medicine and Haematology, University of the Witwatersrand DST/NRF Centre of Excellence for Biomedical Tuberculosis Research

Resuscitation promoting factors (Rpfs) are a group of enzymes that have been implicated in reactivation of dormant bacteria via a mechanism involving hydrolysis of peptidoglycan and have been investigated in Mycobacterium tuberculosis for their possible contribution to tuberculosis disease. In this study, we characterized four Rpf homologues (rpfA, rpfB, rpfE and rpfE2) in a 
non-pathogenic relative, Mycobacterium smegmatis. We demonstrated that these genes were differentially expressed during axenic growth however, rpfE2 appeared to be highly induced during biofilm formation. We further characterized deletion mutants that lacked rpfA, rpfB, rpfE 1 and rpfE2, singularly or in different combinations thereof. Single deletion mutants were phenotypically indistinguishable from the wildtype. In contrast any combinatorial deletion that lacked both rpfA and rpfB resulted in the loss of surface colony cording, in addition to the inability to form mature biofilms. Scanning electron microscopy revealed that while bacterial cells within the defective biofilms were "packing" similarly, a significant reduction in cell length was observed. Exogenous supplementation of Rpf (either from the supernatant of a biofilm or axenic culture of wildtype) failed to the correct the biofilm defect, suggesting that de novo Rpf biosynthesis is required during biofilm formation. Moreover, strains defective for biofilm formation were shown to be more susceptible to killing by rifampicin, a phenotype which was reversed following genetic complementation. Collectively, our data prove that despite the dispensability of all four Rpf homologues in $M$. smegmatis in vitro, a functional divergence exists within this gene family with respect to cellular morphology and biofilm formation.

Key words: Mycobacteria, Rescusitation-Promoting Factor, Biofilm

\section{MCB-0-6: THE WNT PATHWAY IN SYSTEMIC SCLEROSIS}

Jacqueline Frost ${ }^{1,2}$, Michelle Ramsay 3 , Mohammed Tikly4, Xavier Estivill ${ }^{5}$

'School of Pathology, Department of Human Genetics, ${ }^{2}$ School of Clinical Medicine, ${ }^{3}$ Sydney Brenner Institute for Molecular Biosciences, University of the Witwatersrand, ${ }^{4}$ Department of Rheumatology, University of the Witwatersrand, ${ }^{5}$ Centre for Genomic Regulation, Barcelona, Spain

Systemic sclerosis (SSC) is a complex autoimmune disease involving the immune system, vasculature and extracellular matrix. Dysregulation of the Wnt pathway has been implicated in the development of fibrosis in SSC and is proposed to contribute to a failure to maintain tissue homeostasis and immune function. The objective of this study was to explore the role of altered Wnt pathway gene regulation in the development of fibrosis in black South African SSc patients. Skin biopsies were taken from eight black South African patients with diffuse cutaneous SSc, both affected and unaffected skin, as well as eight healthy controls. The Wnt pathway RT2 Profiler qPCR Array was used to assess differential gene expression, TaqMan assays for validation and SRNA-sequencing for microRNA analysis. Data analysis was done using HTqPCR, NormqPCR and DESeq2 software. Gene expression patterns revealed five distinct differentially expressed gene clusters. Two clusters displayed genes that were upregulated in both affected and unaffected SSc skin compared to controls. Another showed decreased gene expression and two revealed more complex patterns responsible for delineating the patients into two groups. The gene expression was validated for five genes. The sRNA-seq data showed differential expression of 31 miRNAs that target the Wnt pathway genes, including miR-335 and miR204 that are important regulators of normal tissue development. In this group of dcSSc patients, there is differential gene expression of several Wnt pathway genes that delineate the patients into two distinct groups. This could point to differences in disease aetiology leading to distinct clinical outcomes.

Key words: Systemic sclerosis, gene expression, Wnt pathway, fibrosis

\section{MCB-O-7: NON-CODING VARIANTS SEGREGATE WITH DISEASE IN SOUTH AFRICAN FAMILIES WITH KERATOLYTIC WINTER ERYTHEMA (KWE)}

Thandiswa Ngcungcu', Bolan Linghu², Fan Yang², Edward Oakeley ${ }^{2}$, Frank Staedtler ${ }^{2}$, Robert Bruccoleri' ${ }^{2}$, Marc Sultan ${ }^{2}$, Joseph Szustakowski², Michele Ramsay ${ }^{1,3}$

'School of Pathology, Department of Human Genetics, Faculty of Health Sciences, University of the Witwatersrand, ${ }^{2}$ Biomarker Development, Translational Medicine, Novartis Institutes for BioMedical Research, Basel, Switzerland and Cambridge, USA, ${ }^{3}$ Sydnney Brenner Institute for Molecular Bioscience, University of the Witwatersrand, Johannesburg, South Africa

Keratolytic Winter Erythema (KWE) is a rare skin disorder of unknown aetiology that is characterized by erythema, keratin layer thickening and centrifugal skin peeling of the palms and soles, especially in cold and dry conditions. KWE shows an autosomal dominant mode of inheritance and the causal mutation was localised to chromosome 8p23.1-p22 (KWE critical region). The aim of this study was to identify and characterize the causal mutation for KWE in South African families. Targeted resequencing (8p23.1-22) and exome sequencing were performed in 21 affected and 28 unaffected individuals using Illumina HiSeq and MiSeq sequencing platforms. Reads were aligned to the reference genome using BWA. GATK and PINDEL were used to call small and large structural variants, respectively. We identified a $7.67 \mathrm{~kb}$ tandem duplication within the KWE critical region, upstream of the CTSB gene and encompassing two enhancers. This tandem duplication was validated in all affected individuals, highlighting the potential role of large structure variants for manifesting Mendelian diseases. The fact that this variant is located in a non-coding region suggests that the tandem duplication may result in altered gene expression regulation. Further functional analyses are required, but this finding enforces the need to look beyond the exome when trying to identify causal mutations for a subset of Mendelian diseases.

Key words: KWE mutation 


\section{MCB-0-8: POSTERIOR SEGMENT COLOMBA AND CONGENITAL CATARACT IN A SOUTH AFRICAN FAMILY}

Saadiah Goolam', Nadia Carsterns², Mark Ross ${ }^{3}$, David Bentley $^{3}$, Margarida Lopes ${ }^{3}$, John Peden ${ }^{3}$, Trevor Carmichael ${ }^{4}$, Michele Ramsay ${ }^{2,}$, Susan Williams ${ }^{4}$

'School of Clinical Medicine, Department of Neurosciences University of the Witwatersrand, ${ }^{2}$ Sydney Brenner Institute for Molecular Bioscience, University of the Witwatersrand, 3llumina Cambridge Ltd,Saffron Walden, United Kingdom, ${ }^{4}$ Department of Ophthalmology, University of the Witwatersrand, ${ }^{5}$ Department of Human Genetics, National Health Laboratory Service, Faculty of Health Sciences, University of the Witwatersrand

Congenital cataracts are an important treatable cause of childhood visual disability. Non-syndromic hereditary forms may be associated with other ocular anomalies in approximately $15 \%$ of cases, usually involving the anterior segment. Inherited forms of coloboma are less frequent than the sporadic form, and are predominantly associated with systemic disease. They may also be associated with other ocular anomalies. Cataracts are much more commonly associated with iris colobomas than isolated posterior segment colobomas. The objective of this study is to report the uncommon phenotype of congenital cataract and posterior segment coloboma in a South African family and to investigate its genetic etiology. Twenty-six family members were included, with 20 individuals affected in an inheritance pattern consistent with autosomal dominant. Posterior segment coloboma (predominantly optic disc coloboma) was present in 17 individuals and iris coloboma in 1. Fourteen had evidence of cataract, with anterior polar cataracts in 10. Nystagmus was present in 18. Other features included high myopia in 8 individuals and microcornea in 3 individuals. DNA was extracted and whole genome sequencing was performed on six affected family members. Variant ranking identified an R208W PAX6 mutation (dbSNP rs757259413; HGMD CM930572) that was present in all 6 sequenced individuals. The R208W PAX6 mutation known to be associated with aniridia is implicated in the variably expressed cataractcoloboma phenotype seen in this family. This association has not previously been described and should contribute to our understanding of the role PAX6 plays in ocular development and cataractogenesis.

Key words: Coloboma, Congenital Cataract, PAX6

\section{MCB-O-9: CHARACTERIZATION OF NONTYPEABLE CARRIAGE PNEUMOCOCCI IN SOUTH AFRICA IN THE CONJUGATE VACCINE ERA, 2009-2012}

Thabo Mohale', Nicole Wolter', Mushal Allam², Susan A. Nzenze², Shabir A. Madh2i, Mignon du Plessis, Anne von Gottberg ${ }^{2}$

'School of Pathology, Department of Clinical Microbiology and Infectious Diseases, Respiratory and Meningeal Pathogens Research Unit, University of the Witwatersrand, 1 Modderfontein road, Sandringham, ${ }^{2}$ National Institute for Communicable Diseases, University of the Witwatersrand
Nontypeable pneumococci (NTPn), not targeted by pneumococcal conjugate vaccines (PCV), commonly colonize the nasopharynx and serve as a reservoir of genetic material for the species. In South Africa, PCV7 was introduced in 2009. We characterized NTPn carriage isolates pre and post PCV7. NTPn isolated from crosssectional carriage studies among participants of all ages in rural (2009 and 2011) and mother-child pairs in an urban community (2010 and 2012) were characterized by multilocus sequence typing, sequence analysis of the capsule and acquisition of antibiotic resistance genes. NTPn increased post PCV7 from $3.3 \%(28 / 853)$ to $4.2 \%(23 / 543)$ $(P=0.38)$ and from $2.7 \%(32 / 1162)$ to $4.6 \%(54 / 1163)(P=0.02)$ in the rural and urban settings, respectively. 42/137 (31\%) NTPn were co-carried with encapsulated strains, 13/137 (9\%) contained partial capsular genes (Group I) and had sequence types (ST) associated with encapsulated strains, and 124/137 (91\%) had complete capsular locus deletion or replacement by non-capsular genes (Group II). Group II were predominantly ST9810 (25/106, 24\%), ST981 1 (13/106, $12 \%)$ and clonal complex 344 (34/106,32\%), and were further classified as null capsule clade (NCC) 1 (5/124,4\%), NCC2a (103/124, 83\%), NCC2b (8/124, 6\%), NCC3 (4/124, $3 \%)$ and NCC4 (4/124,3\%). 50/137 (36\%) NTPn contained ermB, 15 (11\%) mefA, 9 (7\%) tetM and 64 (47\%) contained $\geq 1$ resistance gene. We observed a small increase in NTPn post PCV7. Majority of our isolates were Group II-NCC2a, of which approximately half were related to PMEN clones NorwayNT-42 and USANT-43.

Key words: Streptococcus pneumoniae, Nontypeable pneumococci, carriage

\section{MCB-O-10: VARIATION IN BONE DENSITY IN THE HUMAN MANDIBLE DURING THE EARLY STAGES OF GROWTH AND DEVELOPMENT}

Erin Hutchinson ${ }^{1,2}$, Mauro Farella ${ }^{3}$, Jakobus Hofmann4, Beverley Kramer

'School of Anatomical Sciences, Faculty of Health Sciences, University of the Witwatersrand, ${ }^{2}$ Centre for Health Policy, ${ }^{3}$ Discipline of Orthodontics, Department of Oral Sciences, Sir John Walsh Research Institute, Faculty of Dentistry, University of Otago, Dunedin, New Zealand, ${ }^{4}$ Radiography and tomography division of Radiation Sciences, South African Nuclear Energy Corporation, Pelindaba, South Africa

During growth, the mandible accommodates increases in biomechanical loading, which may arise from dental development, cycles of nutritive and non-nutritive suckling and the muscles of mastication. While these changes in loading are thought to significantly alter bone density, little information on these changes exist in the literature. Thus, this study aimed to evaluate changes in bone density across the body of the immature mandible during the pre- and postnatal periods of growth. The study sample included 45 mandibles, sub-divided into three age groups: 
prenatal (30 gestational weeks to birth), early postnatal (birth to 12 months) and late postnatal (1 to 5 years). Mandibles were scanned using X-ray micro-CT. Eight points were selected along the external (labial/buccal) and lingual surfaces of each dental crypt. Density ratios were calculated using a reference standard. Statistical analyses included ANCOVA and MANOVA. The density of the lingual surface was significantly higher $(p<0.000)$ than the external surface. Bone density on the alveolar buccal surface of the central incisor $(p<0.000)$ and the deciduous molar $1(p<0.013)$, as well as the lingual alveolar area of deciduous molar 2 ( $p<0.032$ ) was significantly greater in the early postnatal period than in the prenatal period. The basal bone on the buccal surface was significantly greater in the later postnatal period. The greater bone density across the postnatal periods may be attributed to the development and eruption of the dentition. Thus, a change in biomechanical demands on the mandible associated with dental development, appear to have an effect on the associated bone density.

Key words: Bone density, immature mandible, alveolar, basal

\section{MCB-O-11: DETERMINING THE MOLECULAR BASIS OF SPINAL MUSCULAR ATROPHY (SMA) IN THE BLACK SOUTH AFRICAN POPULATION}

Elana Vorster', Amanda Krause ${ }^{2}$, Fahmida Essop ${ }^{2}$

'School of Pathology, Department of Human Genetics, University of the Witwatersrand, National Health Laboratory Service, ${ }^{2} \mathrm{NHLS}$, Wits

SMA is a common autosomal recessive, neuromuscular disorder, characterised by muscle atrophy and impaired mobility. A homozygous deletion of the SMN1 gene, exon 7 (SMN1-EX7) is the main cause of SMA in $~ 95 \%$ of patients worldwide but only accounts for $51 \%$ of SA black patients. SMN1 and a highly homologous pseudogene, SMN2, are located in a complex duplicated region. Unusual copy number variations (CNVs) have been reported in black patients, suggesting the presence of complex pathogenic rearrangements. The aim of this project is to investigate the disease mechanism in the black SA population further. MLPA testing was performed on 172 unrelated black patients referred for SMA testing. Of these, 50 had a homozygous SMN1-Ex7 deletion, 50 had a homozygous SMN2-Ex7 deletion and 72, with a strong clinical suspicion of SMA, had no homozygous deletions. Furthermore, 122 black negative controls were tested. For comparison, 68 white patients (38 SMA patients and 30 negative controls) were tested. No clear pathogenic CNV pattern was identified in black patients. Only $8.3 \%(6 / 72)$ of patients with a strong clinical suspicion had a heterozygous deletion of SMN1-EX7 which is lower than previous SA reports of $70 \%$. Multiple (>2) copies of SMN1-EX7 were observed in $50.8 \%$ (62/122) of black negative controls. This study re-emphasises the hypervariability and the limited understanding of CNVs at the SMN region in African populations. While MLPA has been shown to be an appropriate technique to identify SMA carriers in the white population, it is not valuable in identifying carriers and potential pathogenic rearrangements in the black population.

Key words: Neurology, Genetics, Copy Number Variation (CNV), Spinal Muscular Atrophy (SMA)

\section{MCB-O-12: SOUTHERN AFRICAN HUMAN GENOME PROGRAMME: DEEP WHOLE GENOME SEQUENCING PROVIDES INSIGHTS INTO THE GENETIC ARCHITECTURE OF SOUTH AFRICANS}

Anayo Choudhury, Scott Hazelhurst, Michele Ramsay Sydney Brenner Institute for Molecular Bioscience (SBIMB)

The pilot study of the Southern African Human Genome Program (SAHGP) includes 24 high coverage whole genome sequences from three Southern African ethnolinguistic groups (Sotho-speakers: SOT, Xhosa-speakers: XHS and Coloured/admixed individuals: COL). Whole genome sequencing (>30X) was performed on the Illumina platform. The discovery of $\sim 0.8$ Million novel SNPs in this small group of 24 individuals confirms the anticipated potential for novel SNP discovery in Southern African populations. Despite the relatively recent divergence of the Southeastern Bantu-speakers (SEB), the two groups (SOT and XHS) were found to be distinguishable in the Principal Component Analysis. Moreover, analyses based on ADMIXTURE showed these populations to also differ significantly in ancestral component contributions. Characterization of Khoesan (KS) ancestry in the Southern African groups show that, in addition to clear variations in the extent of Khoesan (KS) ancestry, the possible source of KS ancestry also differs between the SEB and COL populations. The Southern African groups were found to show extreme variation in frequency and length of runs of homozygosity ( $\mathrm{ROH}$ ) segments, with the longest and most numerous ROH blocks in Southern African KS groups and the Niger-Congo speaker groups, including the SOT and XHS. In accordance with their history of recent and complex admixture, the shortest and smallest number of $\mathrm{ROH}$ blocks was observed in the COL. We highlight the need to study different African populations to understand their unique patterns of genetic diversity, to discover novel genetic variation and to promote biomedical research on the sub-continent.

Key Words: Whole Genome Sequence, Novel SNPS, Admixture, Population Structure 


\section{MCB-O-13: THE REPRESENTATION OF INDIAN GENETIC DIVERSITY IN THE 1000 GENOMES PROJECT DATASET}

Dhriti Sengupta', Ananyo Choudhury', Analabha Basu², Michele Ramsay'

ISydney Brenner Institute for Molecular Bioscience, University of Witwatersrand, Johannesburg, South Africa, ${ }^{2}$ National Institute of Biomedical Genomics, Kalyani, India

Genomic variation in Indian populations is of great interest due to the diversity of ancestral components, social stratification, endogamy and complex admixture patterns. With an expanding population of 1.2 billion, India is also a treasure trove to catalogue innocuous as well as clinically relevant rare mutations. Recent studies have revealed four dominant ancestries in populations from mainland India; Ancestral North-Indian (ANI), Ancestral South-Indian (ASI), Ancestral Tibeto-Burman (ATB) and Ancestral Austro-Asiatic (AAA). The 1000 Genomes Project (KGP) Phase 3 data include about 500 genomes from 5 linguistically defined Indian-Subcontinent (IS) populations (Punjabi, Gujrati, Bengali, Telugu and Tamil) some of whom are recent migrants to the USA or UK. Comparative analysis show that despite of the geographic spread of the KGP-IS populations, only the ANI component is predominantly represented in this dataset. Previous studies have reported population substructure in the HapMapGujrati population. Our study confirms the substructure in the Gujrati population, and shows evidence for similar substructure in the Punjabi and Telugu populations. Moreover, we demonstrate that this structure is unlikely to be due to novel ancestry, as suggested in previous studies; but more likely to be a consequence of a combination of the following: differences in the proportion of ancestral components and social structure. Our analyses suggest that using language and/or geography as a proxy for an ethnic unit is inadequate for many of the IS populations and highlights the necessity of more nuanced sampling strategies, particularly for biomedical research.

Key words: Indian genomic diversity, 1000 Genomes Project, Population structure, Inbreeding coefficient

\section{MCB-O-14: FBN1 MUTATIONS IMPLICATED IN PRIMARY CONGENITAL GLAUCOMA IN A SOUTH AFRICAN FAMILY}

Susan Williams ${ }^{1,2}$, Nadia Carstens', Saadiah Goolam', Scott Hazelhurst', Trevor Carmichael', Michele Ramsay ${ }^{2}$

'School of Clinical Medicine, Department of Ophthalmology, ${ }^{2} S y d n e y$ Brenner Institute for Molecular Bioscience, ${ }^{3}$ Department of Human Genetics, University of the Witwatersrand, ${ }^{4}$ School of Electrical and Information Engineering, University of the Witwatersrand

Primary congenital glaucoma (PCG), an important cause of childhood blindness, has known genetic associations with CYP1B1 and LTBP2, however mutations in these genes are uncommon in South Africans with PCG. The aim of this study was to identify the cause of PCG in an admixed South African family with two affected children. The two affected children were phenotyped and samples were taken from them, both their parents, and their unaffected siblings for DNA extraction and genetic analysis. Whole exome sequencing on the six family members was used for mutation identification. Variant filtering included the inheritance pattern, variant minor allele frequency and potential functional impact. Both affected children had isolated bilateral primary infantile glaucoma. Both were found to be osteopenic on bone densitometry. Light and electron microscopy on skin samples revealed extracellular matrix abnormalities compared to controls. There were no potentially pathogenic homozygous mutations identified within the exome by variant filtering. Potential compound heterozygous mutations were identified and evaluated in FBN1, PCDHGB7, PER2 and SLC25A48. The most likely causal gene was $\mathrm{FBN} 1$ that contained two extremely rare splicesite mutations that segregated with PCG in this family. FBN1 codes for the ubiquitous extracellular matrix glycoprotein, fibrillin-1 and is an excellent biological candidate gene for PCG because of its regulatory effects on the extracellular matrix and its interaction with LTBP2. Our findings could potentially increase current understanding of the disease mechanisms underlying PCG.

Key words: Primary, congenital, glaucoma, genetics

\section{MCB-O-15: BREAST CANCER IN HIGH- RISK AFRIKANER FAMILIES: IS BRCA FOUNDER MUTATION TESTING SUFFICIENT?}

Tasha Wainstein, Heather Seymour, Shelley Macaulay, Tabitha Haw, Amanda Krause

School of Pathology, Department of Human Genetics, Faculty of Health Sciences, University of the Witwatersrand

Germline mutations in BRCA1/2 genes result in Hereditary Breast and Ovarian Cancer syndrome. In the Afrikaner population of South Africa, three founder mutations in the BRCA genes have been identified. The aim of this study was to investigate the uptake and type of molecular testing performed on patients, to determine the prevalence of the Afrikaner founder and other non-founder BRCA mutations in the study population, and to analyse the utility of two mutation prediction models (Breast and Ovarian Analysis of Disease Incidence and Carrier Estimation Algorithm (BOADICEA) and Manchester scoring method) in assisting with the decision for the most cost-effective testing option. A retrospective file review was performed on counsellees of self-reported Afrikaner ancestry from Johannesburg (2001-2014), with a personal or family history of breast and/or ovarian cancer. Demographic and family history information was recorded and Manchester and BOADICEA scores were calculated for each patient. Of 
86 unrelated counsellees, 54 (62.8\%) underwent BRCA genetic testing; 18 (33.3\%) tested positive for a mutation, and 14 of these $(77.8 \%)$ for an Afrikaner founder mutation. Four non-founder mutations were identified. BOADICEA scores were significantly higher in counsellees who tested positive for a mutation than in those who tested negative. Founder mutation testing should be performed as a firstline option. BOADICEA is useful in identifying counsellees at high risk and assists with the decision to pursue further testing following a negative founder mutation result. These findings assist in guiding an informed genetic counselling service for at-risk individuals with an Afrikaner background.

Key words: Breast cancer, Afrikaner, Founder, Genetic Testing

\section{MCB-0-16: SOUTH AFRICAN JUVENILE FACIAL SOFT TISSUE THICKNESS DATA IN FACIAL APPROXIMATIONS: A COMPARATIVE STUDY}

\author{
Nanette Briers, Maryna Steyn
}

School of Anatomical Sciences, University of the Witwatersrand

In the forensic field, facial approximation is used to predict a face from a skull of which the identity is unknown. Appropriate facial soft tissue thickness (FSTT) data based on ancestry, age and sex are used in order to produce an approximation that may be recognised by family and friends. The lack of knowledge regarding South African juvenile FSTT standards of particular age groups, sex and ancestry is problematic. The study aimed to determine and compare South African juvenile FSTT data of different age groupings to British, American, Canadian, Turkish and Japanese children, as well as the internationally pooled T-tables.In total, 388 cephalograms of South African children, aged 6 to 13 years, were digitized. FSTT measurements were taken at ten mid-facial landmarks using the iTEM measuring program, followed by statistical analysis.Results showed that FSTT of South African children were significantly smaller than American and Japanese children as well as FSTT reported in the international T-tables. In contrast, FSTT was larger in the South African sample and differed at most landmarks from British and Turkish children in the lower face region. The FSTT for Canadian children at the forehead and lower face region were larger than for South African children.This study provides specific FSTT data to increase the accuracy of facial approximations in local juvenile cases. In addition, juvenile FSTT data should consider the onset of puberty and ancestry, but not sex as the latter seem not to impact FSTT with more than $2 \mathrm{~mm}$ at any landmark.

Key words: Facial approximation, facial soft tissue thickness, craniofacial identification

\section{MCB-O-17: DESIGNING AN EFFICIENT GENOME-WIDE GENOTYPING ARRAY SPECIFIC TO AFRICAN POPULATIONS - THE H3AFRICA CUSTOM CHIP CONSORTIUM}

Zane Lombard', Adebowale Adeyemo², Nicola Mulder ${ }^{3}$

'School of Pathology, Department of Human Genetics, ${ }^{2} \mathrm{National}$ Institutes of Health, Bethesda, MD, USA, ${ }^{3}$ Computational Biology Division, Department of Integrative Biomedical Sciences, IDM. University of Cape Town Cape Town, South Africa, Members of the H3Africa custom African chip consortium.

H3Africa (Human Heredity and Health in Africa) is a partnership among the National Institutes of Health (USA), the Wellcome Trust (UK), and the African Society of Human Genetics. The objective of H3Africa is to facilitate a contemporary research approach to the study of the genomic and environmental determinants of disease in Africa, and in such to improve the health of African populations. Currently available genotyping arrays are not ideally suited to studying African populations, therefore the main aim of the H3Africa custom chip consortium is the design of a cost-effective genomewide association (GWAS) array, with content appropriate for use in genomic studies of individuals from the African continent. To enable this, a large sequence reference panel (based on whole-genome sequence data) of African populations will be designed, and used to select an appropriate GWAS scaffold for the array. Both publicand private sequence data will be used in this effort. All data will be curated and merged into a unified dataset. Subsequently, a reference panel will be generated by genotype refinement and phasing. Haplotypes from the reference panel will then be used to select tagSNPs for the chip design using a hybrid approach. This includes using a multi-population tagging algorithm, as well as imputation. Only common SNPs (minor allele frequency $>5 \%$ ) will be tagged for inclusion on this array. The designed GWAS scaffold will be supplemented with other valuable array features (like copy number probes and known functional variants) to result in a final array with 2.5 million probes.

Key words: Genome-wide association study, genotyping, array

\section{MCB-P-1: POPULATION DISTRIBUTION OF FORENSICALLY IMPORTANT DIPTERA ON THE GAUTENG HIGHVELD}

Allison Gilbert, Richard Hunt, Maureen Coetzee School of Pathology, Department of Vector Control Reference Laborator, Wits Research Institute for Malaria (WRIM)

The Calliphoridae are a group of medically important flies which cause myiasis and can be used for forensic investigations. For this group of flies to be practically used in forensic applications, an understanding of the population dynamics of the region is necessary. As there is a lack of genomic data for the numerous fly species, primary research into identification of species on a molecular 
level is required. As there is a lack of robust sequencing data, more basic techniques will be explored to lay the foundations for future molecular species identification. In this study cytogenetic examination of polytene chromosomes as well as isoenzyme electrophoresis were examined as potential identification methods. Cytogenetic studies have displayed potential to serve as a speciation tool, but further work is required. Isoenzyme electrophoresis has demonstrated variable results. Some systems, such as isocitrate dehydrogenase, does not vary between species. Other more polymorphic systems, such as the general esterases, show marked differences in isozyme patterns between species as well as between immature stages and adult flies. The results to date therefore suggest that these techniques can potentially be used as molecular identification tools.

Key words: Calliphoridae; isoenzyme-electrophoresis; cytogenetics

\section{MCB-P-2: GONADOTROPIN-RELEASING HORMONE RECEPTORS WITH MUTATIONS IN TRANSMEMBRANE HELICES 2 OR 6 HAVE SIMILAR PHENOTYPES, SUPPORTING DISRUPTION OF A CONSERVED WATER- MEDIATED HYDROGEN BOND NETWORK}

Ashmeetha Manilall, Colleen Flanagan

School of Physiology, University of Witwatersrand, Receptor Gene Biology

Gonadotropin-releasing hormone (GnRH) binds to $\mathrm{GnRH}$ receptors (GnRHR) in the pituitary where it stimulates release of $\mathrm{LH}$ and $\mathrm{FSH}$, which control reproduction. It had been proposed that a negatively-charged wildtype Glu ${ }^{2.53(90)}$ residue is required to form a salt-bridge with a positively-charged Lys. However, we showed that a negative charge is unnecessary, as mutant GnRHRs with large hydrophobic substitutions for Glu2.53(90) were wellexpressed and had wild-type-like function (Manilall et al., 2014). To confirm this, we mutated Glu $2.53(90)$ to positivelycharged Arg, which was well-expressed, confirming that Glu ${ }^{2.53(90)}$ does not form an ionic interaction to stabilize GnRHRexpression. However, the Glu ${ }^{2.53(90)}$ Arg GnRHR mutant had decreased affinity for $\mathrm{GnRH}$, suggesting that it distorts the ligand binding pocket. The equivalent residue, $\mathrm{Met}^{2.53(86)}$, of rhodopsin interacts with the conserved Trp ${ }^{6.48(265)}$ residue in transmembrane helix 6, via watermediated hydrogen bonding. To test for this interaction in the GnRHR, we mutated Trp ${ }^{6.48(280)}$ to Arg. The Trp.48(280) Arg mutant had decreased affinity for $\mathrm{GnRH}$, similar to the decreased affinity of the Glu ${ }^{2.53(90)} \mathrm{Arg}$ mutant, suggesting a similar distortion of the ligand binding pocket. The similar phenotypes of these mutant GnRHRs suggests that the Glu ${ }^{2.53(90)}$ and the Trp ${ }^{6.48(280)}$ side-chains are located such that they may interact via the water-mediated hydrogen bonding network that is conserved in rhodopsin-like $G$ protein-coupled receptors.

Key words: GnRH, Receptor, Expression, Mutant

\section{MCB-P-3: PATTERN OF DOUBLE CORTIN EXPRESSION IN THE HIPPOCAMPUS OF THE LONG-EVANS RATS EXPOSED TO DIFFERENT ENVIRONMENTAL ENRICHMENT}

Chioma Blessing Uzokwe, Pilani Nkomozepi, Manger Paul, Ihunwo Amadi Ogonda

School of Anatomical Sciences, Department of Anatomy, University of the Witwatersrand

This research was designed to study neural proliferation patterns of adult generated neurons in the dentate gyrus of the hippocampus by comparing brains of Long-Evans rats exposed to three environmental paradigms. The Long-Evans rats were exposed to standard laboratory $(n=6)$, single influencing factor of running wheel exercise $(n=6)$ and complex enriched environment $(n=6)$ for 28 days. Thereafter, the rats were anaesthetized and perfusion fixed with $4 \%$ paraformaldehyde and sectioned at $50 \hat{A} \mu \mathrm{m}$. Cresyl violet staining for cytoarchitecture was followed by doublecortin (DCX) immunohistochemistry and immunofluorescence for the immature neurons with dendritic patterns. Results indicate an increase in brain weight for the complex enriched group when compared to the running exercise and standard group, supporting documented evidence that environmental enrichment increases brain weight. We analysed DCX positive cells for dendritic patterns and neuronal arrangement on the dorsal/ventral limbs of the dentate gyrus and also volumetric analysis of the dentate gyrus which varied significantly among groups. Neurones in the complex environment had more than one primary dendrite arising from the soma which began branching before reaching the molecular cell layer and a more complex appearance of the branching pattern, and cell bodies evenly distributed on the dorsal and ventral limbs of the dentate gyrus. The exercise group presented a distribution of more neurons on the spatial (dorsal) limb than the control group with similar neuron pattern and branching as the control, where neurons had a primary dendrite arising from the soma and extends to the molecular layer before branching into secondary dendrites.

Key words: DCX, neurone, immunohistochemistry

\section{MCB-P-4: THE RELATIONSHIP BETWEEN THE VITAMIN D SYSTEM AND AUTOIMMUNITY IN AFRICAN TYPE 1 DIABETIC PATIENTS.}

David Msibi', Eleanor Cave², Sureka Bhola ${ }^{3}, K^{2}$ atherine Prigge ${ }^{3}$, Marketa Toman ${ }^{3}$, Nigel J Crowther ${ }^{3}$, Carolyn Padoa ${ }^{3}$

'School of Pathology, Department of Chemical Pathology, ${ }^{2}$ University of the Witwatersrand, ${ }^{3}$ University of the Witwatersrand, NHLS

Vitamin D binding protein (VDBP) polymorphisms have been associated with type 1 diabetes (TID). VDBP is essential for transport of vitamin D metabolites. Our aim was to determine if there are associations between 
serum levels or genotypes of the VDBP with TID and associated immunological markers in a South African black population. TID patients $(n=103)$ and controls $(n=97)$ were recruited for this study. Participants were genotyped for two VDBP single nucleotide polymorphisms (SNPs; rs7041 and rs4588) by PCR-RFLP. VDBP levels, GAD65 and IA2 autoantibody positivity were measured by ELISA. Vitamin D concentrations were determined by HPLC. Data was analysed using Statistica. There was no significant difference in VDBP (303 $\hat{A} \pm 140$ vs. $322 \hat{A} \pm 88.7$ $\mathrm{ng} / \mathrm{L} ; \mathrm{p}=0.29)$ and vitamin $\mathrm{D}$ levels $(64.8[52.4,79.6] \mathrm{vs} .61 .3$ $[48.8,76.9] \mathrm{nmol} / \mathrm{L} ; \mathrm{p}=0.20$ ) between patients and controls, respectively. However, BMI was significantly lower in patients than controls $(23.7$ [21.0; 28.0] vs. 26.6 [23.5; 31.6]; $\mathrm{p}=0.0001)$. There were no differences in VDBP SNP allelic frequencies between the two groups. The VDBP levels were lower in GAD-positive than GAD-negative cases (222 $\hat{A} \pm 100$ vs. $283 \hat{A} \pm 122 \mathrm{ng} / \mathrm{L} ; \mathrm{p}=0.03$ ), whilst vitamin $D$ levels were significantly higher in IA2-positive than IA2-negative cases $(86.9[60.7,98.6]$ vs. $61.5[50.5,77.0] \mathrm{nmol} / \mathrm{L} ; \mathrm{p}=0.01)$. In conclusion, autoantibody positivity in African TID subjects is associated with higher systemic levels of vitamin $D$ and lower systemic levels of VDBP. This data suggests that the vitamin $D$ system is linked to the autoimmune process in diabetic subjects, and warrants further investigation.

Key words: TypelDiabete, VitaminD, Autoimmunity

\section{MCB-P-5: EFFECT OF METFORMIN ON ARGININE UPTAKE IN PANCREATIC DUCTAL CANCER CELLS}

Geoffrey Candy, Marietha Nel

School of Clinical Medicine, Department of Surgery, University of the Witwatersrand

Cancer and type 2 diabetes (NIDDM) share risk factors including age, sex, obesity, diet, use of alcohol, smoking and physical inactivity. Low-grade inflammation and oxidative stress from elevated glucose concentrations are also been implicated in the development of cancer. Pancreatic cancer ranks amongst cancers with the poorest outcomes and rapid onset of NIDDM a risk factor for this cancer. Accumulating evidence suggests that the use of metformin, not other drugs used to treat NIDDM, may inhibit cancer cell growth and proliferation. Metformin is believed to inhibit the mTORC1 pathway, the mammalian target of rapamycin complex 1, a central controller of growth which responds to the availability of nutrients. Metformin has a chemical structure similar to arginine. We determined the effect of metformin on arginine uptake in a pancreatic adenocarcinoma cell line. MIA PACA2 cells were grown to confluence, depleted of arginine and exposed to metformin for 30 minutes. 3H-Arginine uptake was then measured over $30 \mathrm{sec}$ and accumulated radiolabel determined by scintillation counting. Metformin enhanced arginine uptake into the cells up to $170 \pm 7 \%$ with a bell-shaped dose-dependency curve on the concentration of both arginine and metformin. The data suggested metformin enhanced arginine uptake by $\mathrm{y}+\mathrm{L}$ rather than $y+$ transport. As dietary arginine increases mTORCl signaling, these data do not explain the beneficial effect of metformin in cancer.

Key words: Pancreatic cancer, arginine, metformin, amino acids

\section{MCB-P-6: FREQUENCY OF SEXUALLY MATURED MALES, IN A WILD ANOPHELES FUNESTUS SWARM IN BURKINA FASO.}

\author{
Zawada, J.W, Dahan-Moss, Y., Dabire, R.,Koekemoer, L.L.
}

School of Pathology, WITS Research Institute for Malaria (WRIM), University of the Witwatersrand

The World Malaria Report stated that approximately 438000 deaths occurred in 2015 where $90 \%$ of these deaths occurred in Africa. The main malaria vector, Anopheles funestus mate through swarming. Newly emerged male mosquitoes need to undergo a physical change prior mating called inversion, which is the rotation of the genitalia between $135^{\circ}$ and $180^{\circ}$. The male genitalia consist of the abdominal segments 8 through 10, which include claspers that are tipped with claws that need to rotate $180^{\circ}$ to enable the male to grasp the female during copulation. Claspers that have not rotated are referred to as " $\mathrm{T}_{0}$ " while " $\mathrm{T}_{4}$ " refers to claspers where the rotated by $180^{\circ}$. There is a limited amount of publications involving the An. funestus species when it comes to the adult male's sexual characteristics and physiology. Collections were made in Bobo-Dioulasso during dusk and dawn periods to maximize the collection of specimens from 3 swarming groups. The rotation of the genitalia was analyzed. All the male mosquitoes were morphologically identified as An. funestus members and members of the An. funestus group were identified to An. funestus species level using a standard multiplex PCR. The frequencies of sexually mature and sexually immature males were compared. These results confirmed that An. funestus undergo sexual maturation and full transcriptome analysis can be used to further elucidate the transcripts associated in this maturation.

Key words: Sexually mature, Anopheles funestus

\section{MCB-P-7: ADDRESSING CANCER TREATMENT IN AN AFRICAN SETTING: A BIOINFORMATICS ANALYSIS OF PHARMACOGENOMICALLY RELEVANT VARIANTS}

Jorge Da Rocha, Michelle Ramsay

School of Pathology, Department of Human Genetics, University of the Witwatersrand

Cancer is a critical health burden in Africa and rates are predicted to double in the next 15 years. Treatments are severe and expensive, and often induce unwanted 
adverse-drug-reactions (ADRs). Due to these ADRs, some patients have a poor treatment outcome. This may be attributed to mutations in genes that encode proteins involved in the metabolism, transport or excretion of the drug and these mutations may vary in frequency among ethnic groups. Many cancer drugs are known to cause ADRs and have warnings which are associated with ethnicity. Most of the research, however, has been done in populations of European, Asian and African-American ancestry, with little data from African populations. The aim of my study is to characterise genetic variants in African whole genome sequence data using bioinformatics tools and approaches. Genes involved in ADRs for major cancer types were identified through the PharmGKB database. The following genes were selected based on the type of cancer and drug categories involved: $A B C B$ 1, DPYD, TYMS, CYP19A1, GSTPI, CYPIB1, CYP3A4, CYP3A5, ESR1, CYP2D6, XRCC1/5, SLC19A1, and SULT1A1/2. Data in the public domain will be mined, including the 1KGP and AGVP whole genome sequence data for 18 African populations. Allele frequencies will be analysed using PLINKV1.91, and functional annotation performed with Ensembl's VEP; PROVEANv1.1.3; Polyphen-2; SIFTv5.2.2 and fathmmv2.3. This approach could lead to the discovery of novel functional genetic variants in African populations and allele frequencies that would highlight the potential public health impact of using specific drugs.

Key words: Bioinformatics, cancer, pharmacogenomics, africans

\section{MCB-P-8: PLATELET-RICH PLASMA INDUCES ALTERATIONS IN ULTRASTRUCTURE AND TGFB1 EXPRESSION IN HORMONE-DEPENDENT MCF-7 BREAST CANCER CELLS.}

Kyrtania Pather, Wendy van der Spuy, Tanya Augustine

School of Anatomical Sciences, Faculty of Health Sciences, University of the Witwatersrand

Breast cancer prevalence is increasing with 200000 new cases diagnosed in the United States in 2014 alone. Many patients with breast cancer will suffer with thromboembolic complications. Therefore the aim of this study was to determine whether platelet-rich plasma (PRP) induces ultrastructural changes in hormone-dependent MCF-7 breast cancer cells, as well as alterations in Transforming Growth Factor $\beta 1$ (TGF- $\beta \neg 1$ ) protein expression in these cells. MCF-7 breast cancer cells were co-cultured with PRP and were fixed accordingly. Results were obtained as electron micrographs (ZEISS ULTRA plus FEG Scanning Electron Microscope) and photomicrographs (Olympus Inverted Fluorescence Microscope) were qualitatively analysed. Photomicrographs were further quantitatively analysed using CellProfiler, IBM SPSS and Statistica. PRPtreated MCF-7 cells showed an epithelial-to-mesenchymal transition (EMT), morphologically. Platelets exposed to MCF-7 breast cancer cells activated the coagulation cascade resulting in fibrin fibre formation between and around breast cancer cells. Platelets at a higher level of activation caused dense fibrin sheet and plaque formation. TGF- $\beta 1$ expression within untreated MCF7cells was seen mainly in the perinuclear cytoplasmic region; however, these expression sites translocated to the edges in the PRP-treated cells. This is indicative of a more aggressive phenotype and is substantiated by quantitative results. which showed an increase in TGF- $\beta 1$ cytoplasmic expression in PRP-treated cells.These results indicate that PRP induces an EMT change in hormonedependent breast cancer cells, advancing the process of metastasis. It is also indicative of hormone-dependent breast cancer cells inducing thrombotic processes, increasing an individuals' risk for thrombosis.

Key words: Breast cancer, Platelet-rich plasma; TGF-B 1, MCF-7 breast cancer cells

\section{MCB-P-9: THE H19/IGF2 IMPRINTED GENE CLUSTER AND ITS ROLE IN BIRTH WEIGHT VARIANCE IN A BLACK, SOUTH AFRICAN COHORT}

Leigh Jacobs, Zane Lombard

School of Pathology, Department of Human Genetics, University of the Witwatersrand

Birth weight is characterised by the World Health Organization as the most definitive indicator of perinatal morbidity and mortality as well as non-communicable disease susceptibility in adulthood. Low birth weight neonates exhibit a 20 times greater health risk than normal or high birth weight infants and with $15 \%$ of Sub-Saharan African newborns weighing less than $2500 \mathrm{~g}$, it is important to understand the genetic and epigenetic etiology of feotal growth and development in a South African context. Current research indicates a parent-of-origin genomic modulation of foetal growth and development through the action of imprinted regions of the genome which show differential allelic expression. However, very little investigation has been conducted on African populations. This study aims to characterise the parental genetic and epigenetic variation within imprinted gene cluster H19/IGF2/ICR1 in 112 black South African parental pairs, for correlation with offspring birth outcomes. Imprinting Control Region 1 (ICRI) on chromosome 11 p15.5 houses imprinted genes IGF2 and H19 which show parent-of-origin expression and are implicated in feotal growth promotion and restriction respectively. 25 single nucleotide polymorphisms (SNPs) within this region will be 
genotyped using TaqMan SNP Genotyping Assays and Inqaba Biosciences Agena MassARRAY system, in order to test for association with birth parameters including birth weight and length, head circumference and ponderal index. Further to this, the methylation status of ICR I will be investigated using quantitative PCR techniques, in order to ascertain if any changes therein affect foetal growth. This study will attempt to illuminate the genomic etiology of birth weight in order to understand the high prevalence of low birth weight infants in the Black, South African population.

Key words: Birth weight, imprinted genes

\section{MCB-P-10: EFFECTS OF NOVEL EGFR INHIBITORS ON BREAST AND COLORECTAL CANCER CELLS.}

Leonie Harmse', Willem Ferdinand Fourie'2, Bennet Forsyth², Allan Prior $^{3}$

'School of Therapeutic Sciences, University of the Witwatersrand, ${ }^{2}$ School of Pharmacy, Department of Pharmacology, University of the Witwatersrand, ${ }^{3}$ School of Chemistry, University of the Witwatersrand

The overexpression and/or over-activity of the epidermal growth factor receptor (EGFR) in many cancers makes it an attractive drug target. EGFR is an autophosphorylating tyrosine kinase that forms a dimer upon ligand binding. Mutated forms of EGFR drive the uncontrolled proliferation of cells in breast-, lung- and colorectal cancer. Using a computational biology approach four novel phenoxyquinazoline inhibitors were designed to specifically bind to the active site of mutated EGFR. The inhibitors were synthesized and analysed for purity by NMR. The compounds were tested against a panel of EGFR expressing and non-expressing cell lines to assess their ability to inhibit cell proliferation and to determine their effects on cell morphology.Both MCF7 breast cancer cells and HT-29 colorectal cancer cells showed the extensive formation of perinuclear vacuoles and a loss of normal nuclear morphology when the cells were treated with the four EGFR inhibitors. HT-29 cells formed structures resembling that of apoptotic bodies. This was confirmed by acridine orange and Hoechst 33342 staining. The IC50 values as determined by the MTT assay varied between 20 and $60 \mu \mathrm{M}$ for both cell lines. Moreover, two of the test compounds caused profound changes in the subcellular localisation of $\mathrm{BAX}$ and $\mathrm{BCl}-2$ in MCF-7 cells. The two proteins co-localized within unusual tubular structures in the cytoplasmic space surrounding the nucleus. In HT-29 cells the effect of the EGFR inhibitors on the cellular distribution of BAX and $\mathrm{BCl}-2$ was different, with only one compound causing the co-localisation as observed in MCF-7 cells.

Key words: EGFR inhibitors, cancer

\section{MCB-P-11: USING CRISPR-CAS9 TO MANIPULATE THE GENOME OF P. FALCIPARUM}

Dale Liebenberg, Theresa L.Coetzer

School of Pathology, Department of Molecular Medicine and Haematology, Wits Research Institute for Malaria (WRIM),

University of the Witwatersrand

The discovery of clustered regularly-interspaced short palindromic repeats (CRISPR) and the development of CRISPR-Cas9 techniques have had a revolutionary effect on genome editing. CRISPR-Cas9 technology is used in this study to investigate the in vivo role of $P$. falciparum inhibitor of apoptosis (IAP) protein (PfIAP; PF3D7_0519600). IAP proteins are important in mammalian programmed cell death (PCD), inhibiting pro-apoptotic caspases. PCD is hypothesised to be used by $P$. falciparum to control its population, although the network of proteins involved in this process needs to be elucidated. The aim of this study is to modify a CRISPR-Cas9 vector to 1) knock-out the pfiap gene and 2) tag the PfIAP protein to localise it under normal and heat-shock conditions, which induce PCD. To delete the pfiap gene the 5' and 3' untranslated regions (UTRs) were cloned into the pL6-GOI vector, together with a targeting single guide RNA (sgRNA) after an appropriate protospacer adjacent motif (PAM) site was identified. PfIAP-GFP tagging involved cloning pfiap homologuos regions and a GFP sequence into the pL6-GOI vector. Another PAM site towards the 3' end of the gene was identified and a new sgRNA was inserted into the vector. $P$. falciparum parasites were transfected with the two vector constructs and following cleavage of the genomic DNA by the Cas9 nuclease at the sites specified by the sgRNA, homologous recombination with the constructs will occur. These studies will enhance our knowledge of parasite biology and may provide a new drug target if the pfiap gene is essential.

Key words: CRISPR-Cas9, Malaria, P. falciparum, Programmed Cell Death

\section{MCB-P-12: GENETIC VARIANTS IN FOUR HYPERCHOLESTEROLEMIA GENES IN AFRICAN POPULATIONS}

Mahtaab Hayat', 2 , Michele Ramsay 2,, Robyn Kerr ${ }^{3}$

'School of Pathology, Department of Human Genetics, ${ }^{2}$ Sydney Brenner Institute for Molecular Bioscience, ${ }^{3}$ Wits

Familial Hypercholesterolaemia (FH) is a common, autosomal dominant, single-gene disorder resulting from haploinsufficiency of the relevant protein. It is characterised by elevated levels of low density lipoprotein (LDL) in the blood. FH is largely asymptomatic as symptoms often present only in the sixth decade and may lead to premature coronary heart disease (CHD) or early death. Early detection and treatment results 
in an increased survival rate as thickening of arteries is delayed. In South Africa, FH has a high prevalence in several founder populations: South African Indians, Afrikaners and Ashkenazi Jews, with well characterised FH causing mutations. Presently, however, there are no data available on prevalence and associated genes in the African populations. This study aims to identify functional genetic variants in African populations in genes known to be associated with $\mathrm{HC}$ : low density lipoprotein receptor (LDLR), apolipoprotein B (APOB), proprotein convertase subtilisin/kexin type 9 (PCSK9) and low density lipoprotein receptor adaptor protein 1 (LDLRAPI). Whole genome sequence data from African participants in the African Genome Variation Project and 1000 Genomes Project will be analysed to identify potentially functional mutations in these FH genes. Single nucleotide polymorphisms (SNPS) and indels will be identified and assessed for loss of function mutations. Two variants with a high functional impact and high frequency in the population will be validated by PCR. The genotypes will be correlated with LDL levels in participants from the AWI-Gen study, including 600 individuals (300 with high and 300 with low LDL levels) in total from six sites in four African countries.

Key words: LDL, cholesterol, African, genes

\section{MCB-P-13: HSPG2 MUTATIONS IDENTIFIED IN BLACK SOUTH AFRICAN CHILDREN WITH PRIMARY CONGENITAL GLAUCOMA}

Nadia Carstens', Susan Williams², Saadiah Goolam², Trevor Carmichael2, Scott Hazelhurst ${ }^{3}$, Michele Ramsay ${ }^{3}$

\section{'School of Pathology, Department of Human Genetics,} University of the Witwatersrand, ${ }^{2}$ Department of Ophthalmology, Department of Neurosciences, University of the Witwatersrand, 3sydney Brenner Institute for Molecular Bioscience, University of the Witwatersrand

Primary congenital glaucoma (PCG), a leading cause of childhood blindness worldwide, is a rare developmental autosomal recessive monogenic disorder characterised by trabecular meshwork dysgenesis. The genetic aetiology of PCG was investigated in 22 black South African PCG cases. Whole exome sequencing was done to an average depth of 43x. Using a tiered approach, we first investigated potentially pathogenic variants in the four known PCG loci, then novel or rare homozygous or compound heterozygous mutations with a high probability of functional impact in the rest of the exome. Sixteen genes predicted by Endeavour to have the highest likelihood of impacting the PCG phenotype are discussed. One boy had a homozygous pathogenic variant (R390H) in CYP $1 \mathrm{~B} 1$ and none of the cases had LTBP2 pathogenic mutations. Eight of the remaining 21 PCG cases had rare, potentially disease causing compound heterozygous variants in the HSPG2 gene, which is located on chromosome 1p36, within a previously identified PCG locus (GLC3B) for which the gene is unknown. Only one of the patients had a mutation in a known PCG gene. We identified potentially disease-causing variants in HSPG2 in eight PCG cases. HSPG2, which encodes perlecan, a microfibril-associated protein involved in cell-adhesion interactions and extracellular matrix integrity, has a reasonable likelihood of impacting the PCG phenotype according to the recently published microfibril hypothesis of glaucoma, which states that defective microfibrils may be an underlying cause of glaucoma. Functional studies are necessary to confirm the effect of perlecan on the PCG phenotype.

Key words: Genetics, glaucoma

\section{MCB-P-14: IMIDAZO[1,2A]PYRIDINES AND NOVEL NUCLEOSIDES CAUSES APOPTOSIS OF COLORECTAL CANCER CELL LINES.}

Nadia Gangat', Jean Dam², Charles de Koning', Leonie Harmse ${ }^{\text {"* }}$

'Department of Pharmacy and Pharmacology, University of Witwatersrand, 7 York Road, Parktown 2193, Johannesburg, South Africa, ${ }^{2}$ School of Chemistry, University of Witwatersrand, Jorissen Street, Braamfontein, 2050, Johannesburg, South Africa.

Worldwide, colorectal cancer is the third leading cause of cancer related deaths. Despite increased understanding of molecular mechanisms of the disease and the introduction of new therapies, the prognosis of metastatic colorectal cancer remains poor. In this study, two colorectal cancer cell lines; HT-29 and DLD-1 were exposed to a new group of nucleoside analogues and imidazo[1,2-a]pyridines aiming to evaluate their cytotoxic activity and apoptosis inducing potential. Cytotoxic activity was determined by the MTT assay. The cell morphology of treated cells were evaluated using the fluorescent vital stains, Hoechs $\$ 33342$, acridine orange and propidium iodide. The annexin $\mathrm{V}$ binding assay was used to determine apoptotic changes to the cell membrane. Of the six compounds, the four imidazopyridines were significantly more active than the nucleoside analogues with $I_{50}$ values below $5 \mu \mathrm{M}$. DLD-1 cells were less sensitive than the HT-29 cells with $I_{50}$ values ranging between 9 and $16 \mu \mathrm{M}$. Two nucleoside analogues were found to be active against both cell lines with $\mathrm{IC}_{50}$ values ranging from 10-22 $\mu \mathrm{M}$. Evaluation of cell morphology showed the presence of membrane blebs and abnormal nuclei including nuclear fragmentation. Exposure of both cell lines to all six compounds showed increased binding of annexin- $V$ to the cell surface, indicative of early apoptosis. In conclusion, the compounds show selective cytotoxicity towards HT-29 and DLD-1 cells. Further research is needed to confirm their potential as lead compounds.

Key words: Colorectal cancer, imidazopyridines, nucleosides 


\section{MCB-P-15: FC GAMMA RECEPTOR III POLYMORPHISMS AND COPY NUMBER VARIATION AS RISK FACTORS FOR SYSTEMIC LUPUS ERYTHEMATOSUS IN BLACK SOUTH AFRICAN PATIENTS}

Nerissa Bloch', Jacqueline Frost', Nimmisha Govind², Mohammed Tikly²

'School of Pathology, Department of Human Genetics, University of the Witwatersrand, Medical School, ${ }^{2}$ School of Clinical Medicine, Division of Rheumatology, University of the Witwatersrand, Medical School

Systemic Lupus Erythematosus (SLE) is a multi-systemic autoimmune disease with varied symptoms and clinical presentations caused by genetic and environmental factors. The genes, Fc gamma receptors IIIA and IIIB, code for the Fc gamma receptors which are cell surface glycoproteins that are involved in the interaction and removal of antigen-antibody complexes from the body into cells. These genes have been shown to be associated with SLE sUsceptibility and disease in previous studies. Determine if single nucleotide polymorphisms (SNPS), allotypes and copy number variation present within the FC gamma receptor genes IIIA and IIIB contribute to the susceptibility of SLE within the black South African population. DNA from 145 black South African SLE patients have been investigated using TaqMan assays to determine SNP differences and CNV number. A PCR has been optimised in order to determine the allotype differences. In past studies, the NA2/NA2 allotype is most strongly associated with SLE in Japanese patients. However, in this study the NA2/NA2 allotype is only present in $14 \%$ of SLE samples (16.7\% in controls) whereas the NA1/ $\mathrm{NA} 1$ allotype is present in $50 \%$ of samples ( $25.3 \%$ in controls). This suggests that the NAl allotype plays a role in SLE in the black South African population. The codominant allotype of $\mathrm{NA} 1 / \mathrm{NA} 2$ does not seem to play as a significant role as the NAl/NAl allotype with it being present in $30 \%$ of SLE samples (50\% in controls).

Key words: SLE, SNP polymorphisms, CNV, Allotypes

\section{MCB-P-16: PHYSIOLOGICAL RESPONSES OF FREE-LIVING AARDVARKS (ORYCTEROPUS AFER) IN THE KALAHARI SEMI-DESERT TO ENVIRONMENTAL STRESS}

Nora Weyer ${ }^{1,2}$, Robyn S Hetem², Mike D Picker, Andrea Fuller ${ }^{2}$, Mike D Picker ${ }^{3}$

'School of Physiology, Brain Function Research Group, ${ }^{2 B}$ Brain Function Research Group, School of Physiology, University of the Witwatersrand, ${ }^{3}$ Animal Demography Unit, Department of Biological Sciences, University of Cape Town

Aardvarks are large, long-lived ( 20 years) nocturnal mammals, widely distributed throughout sub-Saharan Africa but excluded from arid zones. Aardvarks leave their burrows to forage on a specialised diet of ants and termites. In the Kalahari semi-desert aardvarks experience extreme environmental pressures, with high mortality evident during a recent summer drought. Besides the direct effects of heat and aridity, food and water resources become limited when entire colonies of social insect prey crash during drought. As the Kalahari becomes hotter and drier under global climate change, aardvarks will need to adjust their behaviour and physiology to survive. We used implanted data loggers, camera traps and behavioural observations to investigate direct effects of seasonal climatic variation on activity patterns, body temperature, physical condition and time of emergence of seven freeliving aardvarks in the Kalahari over two years. Climatic variables were recorded by an on-site weather station. We also assessed seasonal changes in aardvark diet via scat analysis and prey availability using pitfall traps. Preliminary results indicate large seasonal variation in prey availability, with ants dominating the diet in summer and termites in winter. Food-restricted aardvarks relaxed the precision of thermoregulation, as shown by increased body temperature variability and low $24 \mathrm{~h}$ minimum body temperatures, and foraged earlier in the day. Body temperature and activity patterns therefore provided an index of aardvark stress and well-being. By linking aardvark physiology and behaviour with current fluctuations in prey availability, we aim to predict the competence of aardvarks to buffer future climate change.

Key words: Aardvark, thermoregulation, climate, stress

\section{MCB-P-17: TRANSCRIPTOME ANALYSIS AND GENETIC VARIATION IN SYSTEMIC SCLEROSIS}

Phelelani Mpangase', Michele Ramsay², Scott Hazelhurst ${ }^{2}$ Mohammed Tikly², Jackie Frost ${ }^{2}$

ISchool of Pathology, Department of Human Genetics, Sydney Brenner Institute for Molecular Bioscience, ${ }^{2}$ University of the Witwatersrand

Systemic sclerosis (SSC) is a complex autoimmune disease of the connective tissue characterized by vasculopathy, immune activation and severe fibrosis of the skin and internal organs. A number of candidate-gene, expression profiling and genome-wide association studies have identified genes, single nucleotide polymorphisms and differentially expressed mRNAs and microRNAs (miRNAs) that play a role in SSC. However, the aetiology of the disease remains unclear, especially in the black South African population. The aim of this study is to shed light on the molecular aetiology of SSc in black South African patients by comparatively analysing and exploring differentially expressed mRNA and sRNA (RNA-seq), and examining potential allelic expression using exome data. The RNA-seq data was sequenced from RNA extracted from skin biopsies taken from affected forearms and unaffected backs of nine black South African SSC patients from the Chris Hani Baragwanath Hospital with 
early diffuse cutaneous SSc. Control skin biopsies were obtained from eight healthy individuals. 100bp pairedend and $75 \mathrm{bp}$ single-end reads were produced for mRNA and sRNA sequencing respectively. Using these data, various bioinformatics approaches will be used to identify differentially expressed genes by examining mRNA transcripts and miRNAs, and to search for potential allele-specific expression. Dysregulation of mRNA and miRNAs expression may trigger the onset of SSC and may reveal molecular pathways involved in the development and progression of SSC. The findings of this study will be validated in 50 cases and 50 ethnically and age-matched controls.

Key words: Systemic sclerosis, transcriptome, RNA sequencing, differential expression

\section{MCB-P-18: INVESTIGATING KNOWN CANCER SUSCEPTIBILITY GENES IN A COHORT OF BLACK SOUTH AFRICAN BREAST CANCER INDIVIDUALS}

Reabetswe Pitere, Tasha Wainstein, Fiona Baine, Amanda Krause School of Pathology, Faculty of Health Sciences, University of the Witwatersrand

Black South African females show a unique phenotype of inherited breast cancer (IBC). Previous research conducted in the Division of Human Genetics has shown that mutations in the BRCA 1 and BRCA2 genes, contribute to IBC. In addition, BRCA2 mutations appear to be more frequent than $B R C A 1$ mutations; this is contrary to what is seen in white populations. IBC is predominantly caused by mutations in the BRCA genes, but other genes such as PTEN and TP53 have been shown to contribute to the development of breast cancer. The aim of this study is to improve our current knowledge of IBC in the black South African population. The first phase of the study involved screening 56 black females for two BRCA2 mutations. The C.5771_5774delTTCA has been previously reported as a founder mutation and the c.582G $>\mathrm{A}$ is suspected to be a potential founder mutation. One individual (0.02\%) was found to have the c.5771_5774delTTCA mutation in the BRCA2 gene. The second phase of the study (currently underway) is to perform haplotyping analysis to determine which haplotypes are segregating with disease. The final phase of the study aims to utilise a next generation sequencing approach to target BRCAl and BRCA2, alongside 26 other cancer susceptibility genes, to identify potential pathogenic mutations. Characterising these mutations could assist with future genetic testing and counselling, as well as improved management and treatment options for the black South African population which has been shown to present with a severe and aggressive breast cancer tumour phenotype.

Key words: Breast cancer, BRCA1, BRCA2, black South Africans

\section{MCB-P-19: ANTICARIOGENIC PROPERTIES OF FLAVONE PRESENT IN DODONAEA VISCOSA VAR. ANGUSTIFOLIA}

Sharon Moeno', Thamsanqa Ngabaza², Mrudula Patel ${ }^{3}$ 'School of Oral Health Sciences, Department of Oral Biological Sciences, ${ }^{2}$ Student under supervision, 'Supervisor

The aim of this study was to analyse the phytochemical constituents of $D$. viscosa var. angustifolia (DVA) and establish their beneficial effects against cariogenic bacteria S. mutans. The crude extract from DVA leaves was fractionated into six fractions (Fl-F6) using chromatography. The Minimum Inhibitory Concentrations (MIC) and Minimum Bactericidal Concentration (MBC) were determined. The effect of the six fractions on biofilm formation and acid production were investigated. The most active fraction (F5) was further fractionated, purified, identified and elucidated using GC-MS and NMR. Cytotoxic effect of crude extract and active subfraction was studied using cell lines. Results were analyzed using Wilcoxon rank-sum test (Mann-Whitney). The MIC and $\mathrm{MBC}$ of the fractions and crude extract ranged from 0.39 to $12.5 \mathrm{mg} / \mathrm{ml}$. F5 showed lowest MBC. At $0.2 \mathrm{mg} / \mathrm{ml}$, F5 reduced biofilm formation by $93.3 \%$ and reduced acid production in S. mutans. Subfraction 5.1 showed higher antimicrobial activity compared to the crude extract and F5. Subfraction 5.1 inhibited biofilm formation at both 6 hours ( $94 \%$ reduction) and 24 hours (99\% reduction) which was higher compared to the crude extract $(87 \%$ reduction at $0.78 \mathrm{mg} / \mathrm{ml}$ after 6 hours). Subfraction 5.1 exhibited a higher inhibitory effect on acid production compared to the crude extract. Subfraction 5.1 was identified as, 5,6,8-Trihydroxy-7,4l-dimethoxyflavone. Cytotoxicity analysis of the crude extract and subfraction 5.1 showed IC50 values of $0.09 \mathrm{mg} / \mathrm{ml}$ and $0.03 \mathrm{mg} / \mathrm{ml}$ respectively. DVA produced an anticariogenic constituent, 5,6,8-Trihydroxy-7,4l-dimethoxyflavone and its safety was established, therefore it has potential for use in the oral cavity to prevent dental caries.

Key words: Phytochemical, Dodonaea Viscosa, Bactericidal

\section{MCB-P-20: NEXT GENERATION SEQUENCING TO IDENTIFY VARIANTS AND TO ESTABLISH THE FREQUENCY OF KNOWN VARIANTS IN GENES. IMPLICATED IN TENOFOVIR EFFICACY AND NEPHROTOXICITY}

Sibongile Tshabalala ${ }^{1,2}$, Natasha Beeton-Kempton' ${ }^{6}$, Faheem Seedat ${ }^{7}$, Ebrahim Variava ${ }^{7}$, Neil Martinson ${ }^{7,8}$, Michele Ramsay ${ }^{4,5}$, Dalu Mancama

'School of Pathology, Department of Human genetics, ${ }^{2} \mathrm{CSIR}$, ${ }^{3}$ Biomedical Technologies Group, CSIR: Biosciences Unit, Pretoria, ${ }^{4}$ Department of Human Genetics, University of the Witwatersrand, Johannesburg, 55ydney Brenner Institute for Molecular Bioscience (SBIMB), University of the Witwatersrand, Johannesburg, 'Molecular Diagnostics Group, CSIR: Biosciences 
Unit, Pretoria, ${ }^{7}$ Department of internal medicine, University of the Witwatersrand, Johannesburg, ${ }^{8}$ Perinatal HIV Research Unit, Baragwanath Hospital and University of the Witwatersrand, Johannesburg

Tenofovir (TDF) is currently used in South Africa and is effective in managing HIV infection. However adverse effects such as nephropathies are often reported to occur, ultimately affecting the effectiveness and clinical outcome of such treatment. TDF-associated nephropathy manifests clinically as acute kidney injury. In this study targeted re-sequencing was performed for ADMErelated genes in $40 \mathrm{HIV}$ infected patients from the Soweto catchment area, with the aim to identify, characterize and determine the allele frequencies of polymorphisms in selected genes that are relevant to TDF-based treatment. Allele frequencies were compared to other populations in the literature and in public databases. Variant filtering including frequency (>0.1), function and previous association with TDF outcome was used to select variants for further investigation. A total of 71 variants (predominantly SNPs) were detected in 5 genes $A B C B 1$, $A B C C 2 A B C C 4 A B C C 10$ and SLC22Al. Using NGS, we uncovered evidence for the presence of nine variants that have previously been shown in other populations to be associated with TDF induced nephrotoxicity. In line with studies on other African populations, higher frequencies of several of these variants were observed in our population compared to Western populations, warranting their further investigation in local populations to determine whether there is a genetic contribution to TDF nephrotoxicity which poses a particular concern in South Africa. Funding for this study is primarily provided through a DST support grant (\# V6YET50) and a CSIR parliamentary grant (\# V1YBT96).

Key words: Next generation sequencing, Tenofovir efficacy and Nephrotoxicity

\section{MCB-P-21: THE GENETIC AETIOLOGY OF OESOPHAGEAL CANCER IN SOUTH AFRICAN BLACK POPULATIONS}

Wenlong Chen', Hannah Bye'2, Iqbal Parker ${ }^{3}$, Chantal Babb $^{4}$, Christopher Mathew ${ }^{5}$

'School of Pathology, Department of Human Genetics, University of the Witwatersrand, 'University of London, 3University of Cape Town, ${ }^{N}$ National Cancer Registry, University of the Witwatersrand, ${ }^{5}$ King's College London

Oesophageal squamous cell carcinoma (OSCC) is common in Black populations of the sub-Saharan Africa, with high incidence regions in East and South Africa. Clinical presentation in Africa is late, and treatment is mainly palliative with a very poor prognosis. Various environmental risk factors have been identified, but the possible contribution of genetics to disease risk is an important question, which is unresolved. The aim of this project is to test the hypothesis that genetic variation in the South African Black population contributes significantly to the risk of OSCC. We have begun by testing genetic risk factors for OSCC which have been identified in other populations for association with OSCC in the South African Black population. We have preliminary evidence for association of SNPs at four loci, TGFBR3, PLCEl, CHEK2 and $X P B 1$, with OSCC $(P<0.01)$. We are expanding the power of this study by genotyping the SNPs in a large case-control collection from the Johannesburg Cancer Study using TaqMan semi-automated assays. We will also use the data to test for gene/environmental interactions, and to build a model for risk prediction by combining genetic and environmental risk factors for OSCC.

Key words: OSCC, TGFBR3, PLCEI, TAQMAN

\section{MCB-P-22: IMIDAZO-[1, 2-A]-PYRIDINE INDUCES APOPTOSIS IN K562 AND HL60 LEUKAEMIC CELL LINES}

Zeenat Ismail', Charles de Koning², Jean Dam², Leonie Harmse'

'School of Therapeutic Sciences, Department of Pharmacy and Pharmacology, University of the Witwatersrand, ${ }^{2}$ School of Chemistry, University of the Witwatersrand, Jorissen Street, Braamfontein, 2050, Johannesburg, South Africa

Leukaemia is a prevalent cancer amongst children in South Africa. Therapeutic failure caused by the development of drug resistance to existing treatments and primary drug resistance warrants the search for new lead compounds. In this context, a library of novel imidazopyridine derivatives were synthesized and evaluated for their anti-proliferative activity against leukaemic K562 and HL60 cell lines.Cells were maintained according to standard protocols. Test compounds were synthesised by recombinatorial chemistry. The MTT assay was used to determine cell viability and $I C_{50}$ values. Cytotoxic effects were confirmed by observing morphological changes using the fluorochromes acridine orange, ethidium bromide and Hoechst-33342. Changes to the cytoskeleton were investigated using fluorescent phalloidin. The ability of active compounds to induce apoptosis were evaluated by measuring caspase-3 activity, Annexin $\vee$ binding and cytochrome c location. Eight cytotoxic compounds with no significant toxicity against normal leukocytes were identified. $\mathrm{K} 562$ cells were most sensitive to JD-88 with an IC50 value of $1.87 \pm 0.91 \mu \mathrm{M}$ and HL60 cells were most sensitive to JD-47R with an $\mathrm{IC}_{50}$ value of $2.053 \pm 0.19$ $\mu M$. Treated cells displayed typical apoptotic features including cell-surface blebbing, nuclear fragmentation and apoptotic bodies. Active compounds induced a time-dependent increase in caspase-3 activity and an increase in Annexin- $\mathrm{V}$ binding. Preliminary studies showed leakage of cytochrome $c$ into the cytosol. This study showed that JD-88, JD-34R, JD-37R, JD-46R, JD-47R and JD-49R exhibited anti-proliferative activity with $I C_{50}$ values lower than $5 \mu \mathrm{M}$. This, combined with their ability to induce apoptosis shows that imidazo-[1, 2-a]-pyridines are useful lead compounds for leukaemia.

Key words: Leukaemia, lead compounds, novel, imidazo-pyridines 